\title{
Prospecting for new physics in the Higgs and flavor sectors
}

A dissertation submitted to the

Gradute School

of the University of Cincinnati

in partial fulfillment of the

requirements for the degree of

Doctor of Philosophy

in the department of Physics

of the College of Arts and Sciences

by

Fady Bishara

M.S. University of Cincinnati

May 2015

Committee chair: Jure Zupan, Ph.D. 


\section{Acknowledgements}

This thesis is the culmination of six years of hard work. However, the same amount of hard work would not have reaped this result in a vacuum. Therefore, I would like to take this opportunity to acknowledge some of those who have guided and supported me throughout the process.

First and foremost, I would like to thank my advisor, Jure Zupan, not only for the countless hours we spent discussing physics but also for invaluable career advice. I would also like to thank Roni Harnik, my adoptive advisor for the last two years.

The physics department at the University of Cincinnati where I spent the first four years of my Ph.D. studies provided an incredibly supportive environment. I would particularly like to thank Philip Argyres, Paul Esposito, Alex Kagan, and Rohana Wijewardhana. They were always generous with their time whether to discuss physics or to give advice. And also my colleagues with whom I spent many hours doing homework or studying for the qualifying exams.

The Fermilab theory department provided a truly collaborative environment during the last two years and for this I am grateful. I have spent many hours discussing physics in an office on the third floor or by the coffee machine and I learned a tremendous amount form the scientists, postdocs, students, and visitors there. In particular, I would like to thank Prateek Agrawal, Paddy Fox, and Felix Yu.

Since every project was done in collaboration with other physicists, I would like to thank my collaborators without whom this thesis would not have been possible - their names are listed on the 'Author list' page.

Last, but certainly not least, I would like to thank my wife, Sarah, whose constant support and encouragement made even the toughest days bearable. 


\begin{abstract}
We explore two directions in beyond the standard model physics: dark matter model building and probing new sources of $\mathrm{CP}$ violation. In dark matter model building, we consider two scenarios where the stability of dark matter derives from the flavor symmetries of the standard model. The first model contains a flavor singlet dark matter candidate whose couplings to the visible sector are proportional to the flavor breaking parameters. This leads to a metastable dark matter with $\mathrm{TeV}$ scale mediators. In the second model, we consider a fully gauged $S U(3)^{3}$ flavor model with a flavor triplet dark matter. Consequently, the dark matter multiplet is charged while the standard model fields are neutral under a remnant $Z_{3}$ which ensures dark matter stability. We show that a Dirac fermion dark matter with radiative splitting in the multiplet must have a mass in the range $[0.5,5] \mathrm{TeV}$ in order to satisfy all experimental constraints. We then turn our attention to Higgs portal dark matter and investigate the possibility of obtaining bounds on the up, down, and strange quark Yukawa couplings. If Higgs portal dark matter is discovered, we find that direct detection rates are insensitive to vanishing light quark Yukawa couplings. We then review flavor models and give the expected enhancement or suppression of the Yukawa couplings in those models. Finally, in the last two chapters, we develop techniques for probing $\mathrm{CP}$ violation in the Higgs coupling to photons and in rare radiative decays of $B$ mesons. While theoretically clean, we find that these methods are not practical with current and planned detectors. However, these techniques can be useful with a dedicated detector (e.g., a gaseous TPC). In the case of radiative $B$ meson decay $B^{0} \rightarrow\left(K^{*} \rightarrow K \pi \pi\right) \gamma$, the techniques we develop also allow the extraction of the photon polarization fraction which is sensitive to new physics contributions since, in the standard model, the right(left) handed polarization fraction is of $\mathcal{O}\left(\Lambda_{\mathrm{QCD}} / m_{b}\right)$ for $\bar{B}^{0}\left(B^{0}\right)$ meson decays.
\end{abstract}





\section{Author list}

I would like to acknowledge my collaborators. Without their contributions, this thesis would not have been possible.

chapter 2: Jure Zupan.

chapter 3: Admir Greljo, Jernej Kamenik, Emmanuel Stamou, and Jure Zupan.

chapter 4: Joachim Brod, Patipan Uttarayat, and Jure Zupan.

chapter 5: Yuval Grossman, Roni Harnik, Dean Robinson, Jing Shu, and Jure Zupan.

chapter 6: Dean Robinson. 


\section{Contents}

$\begin{array}{llr}1 & \text { Introduction } & 1\end{array}$

2 Continuous Flavor Symmetries and the Stability of Asymmetric Dark Matter 6

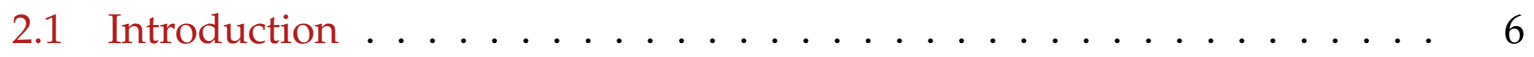

2.2 Dark matter mass in asymmetric dark matter models . . . . . . . . 8

2.3 Metastability and flavor breaking . . . . . . . . . . . . 12

2.3.1 Minimal Flavor Violation . . . . . . . . . . . . . . . . . . . 14

2.3.2 Spontaneously broken horizontal symmetries . . . . . . . . . 17

2.4 Indirect detection . . . . . . . . . . . . . . . . . . . . . . . 19

2.5 Mediator models . . . . . . . . . . . . . . . . . . . . . 21

2.5.1 MFV model with scalar mediators . . . . . . . . . . . 22

2.5.2 FN model with fermionic and scalar mediators . . . . . . . . . 24

2.6 Experimental signatures of the mediators . . . . . . . . . . 26

2.6 .1 Flavor constraints . . . . . . . . . . . . . . 26

2.6.2 Relic abundance and direct detection . . . . . . . . . . . . 30

2.6 .3 Collider signatures . . . . . . . . . . . . . . . . . 31

2.7 Chapter summary . . . . . . . . . . . . . . . . . . . . . . . 35

3 Dark Matter And Gauged Flavor Symmetries $\quad 37$

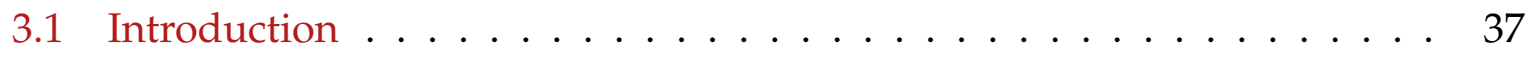

3.2 Stability of flavored dark matter . . . . . . . . . . . . . . . . 39

3.3 Gauged flavor interactions and dark matter . . . . . . . . . . . . 41

3.3.1 Fermionic flavored dark matter . . . . . . . . . . . . . . . . . 44

3.3.2 Scalar flavored dark matter . . . . . . . . . . . . . . . 48

3.4 Dark matter and new physics phenomenology . . . . . . . . . . . . 49

3.4 .1 Scan results . . . . . . . . . . . . . . . . 50

3.4 .2 Thermal relic . . . . . . . . . . . . . . . . 53 
3.4.2.1 Fermionic dark matter . . . . . . . . . . . . . 54

3.4.2.2 Scalar dark matter . . . . . . . . . . . . . . 56

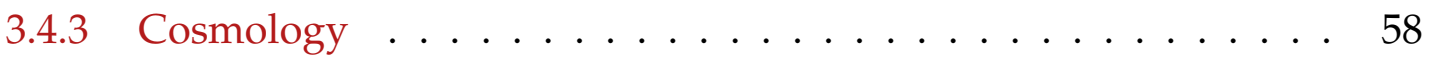

3.4.4 Direct and indirect dark matter searches . . . . . . . . . . . 61

3.4 .5 Searches at the LHC . . . . . . . . . . . . . . . . . 64

3.4 .6 Flavor constraints . . . . . . . . . . . . . . . . . 66

3.5 Benchmarks . . . . . . . . . . . . . . . . . . . . . . . . . 69

3.6 Chapter summary . . . . . . . . . . . . . . . . . 76

4 Nonstandard Yukawa couplings and Higgs portal dark matter $\quad \mathbf{8 0}$

4.1 Introduction . . . . . . . . . . . . . . . . . 80

4.2 Higgs portal with non-trivial flavor structure . . . . . . . . . . . . 82

4.2.1 Annihilation cross sections . . . . . . . . . . . . . . 84

4.2 .2 Relic abundance . . . . . . . . . . . . . . . . . 87

4.2 .3 Invisible decay width of the Higgs $\ldots \ldots \ldots \ldots \ldots$

4.2 .4 Indirect detection . . . . . . . . . . . . . . . . . . . 92

4.2 .5 Direct detection . . . . . . . . . . . . . . . . . . . . . . . 95

4.3 Changes to Yukawa couplings in new physics models . . . . . . . . . . 101

4.3.1 Dimension-Six Operators with Minimal Flavor Violation . . . . 102

4.3.2 Multi-Higgs-doublet model with natural flavor conservation . . 105

4.3.3 Type-II Two-Higgs-Doublet Model . . . . . . . . . . . . . . . . 107

4.3.4 Higgs-dependent Yukawa Couplings . . . . . . . . . . . . . . 108

4.3.5 Randall-Sundrum models . . . . . . . . . . . . . . . . . . . . . 110

4.3.6 Composite pseudo-Goldstone Higgs . . . . . . . . . . . . . . 112

4.4 Constraining the light-quark Yukawa couplings . . . . . . . . . . . . 114

4.5 Chapter summary . . . . . . . . . . . . . . . . . . . . 117

5 Probing CP Violation in $h \rightarrow \gamma \gamma$ with Converted Photons 119

5.1 Introduction . . . . . . . . . . . . . . . . . . . . . . . . 119

5.2 Higgs diphoton decay with CP violation . . . . . . . . . . . . . 123

5.2.1 Motivation for measuring CP-violation in di-photons . . . . . . 123

5.2 .2 Helicity interference . . . . . . . . . . . . . . . . . . 126

5.2.3 A thought experiment with polarizers . . . . . . . . . . . . 127

5.3 Bethe-Heitler photon conversion . . . . . . . . . . . . . . . . . . . . . 129

5.3.1 Photon propagation and conversion . . . . . . . . . . . . . 129

5.3.2 Angular resolution limitations . . . . . . . . . . . 130 
5.3.3 Nuclear form factor . . . . . . . . . . . . . . . . . . . . . . . 132

5.4 The Higgs-Bethe-Heitler process ． . . . . . . . . . . . . . . . . . 134

5.4.1 Amplitude and cross-section . . . . . . . . . . . . . 134

5.4 .2 Helicity structure . . . . . . . . . . . . . . . . . . . . 136

5.4.3 The Bethe-Heitler helicity amplitudes . . . . . . . . . . . . . 139

5.5 Sensitivity to CPV . . . . . . . . . . . . . . . . . . . . . . 142

5.5 .1 Differential scattering rate . . . . . . . . . . . . . . 143

5.5.2 Global sensitivity to CP violation . . . . . . . . . . . . . . . 144

5.5.3 Differential azimuthal scattering rate . . . . . . . . . . 146

5.5 .4 CPV enhancing cuts . . . . . . . . . . . . . . . . . 148

5.5.4.1 Cuts on collective kinematics . . . . . . . . . . . . . 149

5.5.4.2 Cuts on individual photon conversions . . . . . . . . 151

5.5.4.3 Cut scheme efficiencies . . . . . . . . . . . . . . 153

5.5 .5 Triple products . . . . . . . . . . . . . . . . 155

5.6 Chapter summary . . . . . . . . . . . . . . . . . . . . . . . 157

6 Probing the photon polarization in $B \rightarrow K^{*} \gamma$ with conversion 159

6.1 Introduction . . . . . . . . . . . . . . . . . . . . . . . 159

6.2 Amplitudes . . . . . . . . . . . . . . . . . . . . 162

6.2.1 Amplitude factorization . . . . . . . . . . . . . . 162

6.2 .2 Kinematics . . . . . . . . . . . . . . . . . . . . . . . . 164

6.2 .3 Helicity amplitude factors . . . . . . . . . . . . . . 165

6.2 .4 Full Amplitude . . . . . . . . . . . . . . . . . . . . . . . 169

6.3 Observables . . . . . . . . . . . . . . . . . . . 170

6.3.1 Differential rate . . . . . . . . . . . . . . . . . . . . 170

6.3.2 Polarization and weak phase observables . . . . . . . . . . . 173

6.3.3 Statistics and Sensitivity Enhancements . . . . . . . . . . . 175

6.4 Simulations . . . . . . . . . . . . . . . . . 177

6.4.1 Relative Oscillation Amplitude . . . . . . . . . . . . . . 177

6.4 .2 Statistics Enhancements . . . . . . . . . . . . . . . . . 179

6.5 Chapter summary . . . . . . . . . . . . . . . . . . . . . . 181

$\begin{array}{llr}7 & \text { Conclusion } & 183\end{array}$ 
A Appendix to Chapter $2 \quad 185$

A.I Operators in four component notation . . . . . . . . . . . . 185

A.II Asymmetric DM relic density . . . . . . . . . . . . . . . . . 186

A.III Calculation of the DM decay time . . . . . . . . . . . . . . . . . 191

A.IV Loop functions in neutral meson mixing . . . . . . . . . . . . . . . . . 194

B Appendix to Chapter $3 \quad 197$

B.I Minimal flavour violation with gauged flavor symmetries . . . . . . . . 197

B.II Thermal relic computation . . . . . . . . . . . . . . . . 200

B.III Higgs coupling Feynman rules . . . . . . . . . . . . . . . 205

C Appendix to Chapter 5 206

C.I Spinor-helicity formalism . . . . . . . . . . . . . . 206

C.II BH spin-helicity amplitudes . . . . . . . . . . . . . . . . . . 209

C.III Polarization-decomposed HBH rate . . . . . . . . . . . . . . . . . 212

C.IV Numerical simulations of Bethe-Heitler conversion . . . . . . . . . . . . 214

C.V Analysis for $q \bar{q} \rightarrow \gamma \gamma \ldots \ldots$. . . . . . . . . . . . . . 215

C.VI Monte Carlo numerical schemes . . . . . . . . . . . . . . . . . . 218

D Appendix to Chapter 6 222

D.I The $B \rightarrow K \pi e^{+} e^{-}$squared matrix element by polarization decomposition 222

D.II $B$ rapidity distribution . . . . . . . . . . . . . . . 223

D.III The Monte Carlo event generator . . . . . . . . . . . . . . . . . . . . . 224

Bibliography 259 


\section{List of Figures}

2.1 Contours of the ADM mass $m_{\chi}$ (in $\mathrm{GeV}$ ) required to obtain the correct $\mathrm{DM}$ relic density as a function of $\left[(B-L)^{2}\right]_{\mathrm{NP}},\left[Y^{2}\right]_{N P}$, i.e. the $(B-L)^{2}$, and $Y^{2}$ summed over effective d.o.f. in visible NP sector, while keeping $[Y(B-L)]_{N P}=\sqrt{\left[(B-L)^{2}\right]_{\mathrm{NP}}\left[Y^{2}\right]_{N P}}$ and $g(B-L)_{\chi}=4$. The visible sector with only the $\mathrm{SM}$, Eq. (2.2), is denoted by a star. . . . . . . . . .

2.2 Feynman diagram for the decay of DM with $B=1$ assuming MFV. This amplitude leads to the partial decay width $\Gamma_{\chi}^{(1)}$ in Eq. (2.13). . . . . . . .

2.3 The solid blue (red dashed) line denotes the $B=2$ DM lifetime as a function of $m_{\chi}$ for the MFV (FN) case, fixing the NP scale to $\Lambda=1(3) \mathrm{TeV}$. Assuming the dominance of one decay mode, the green (orange) line shows the constraint on the decay time from FERMI-LAT [70] for $b \bar{b}$ $\left(\mu^{+} \mu^{-}\right)$final states using the NFW profile. The dash-dotted red line shows the AMS-02 [71] constraint on $\chi \rightarrow \mu^{+} \mu^{-}$decay time derived in [72], while the light blue line shows the Super-Kamiokande [73] constraint on the $\chi \rightarrow \nu \bar{\nu}$ decay time obtained in [74]. The purple line shows the upper limit on $\chi \rightarrow u d s$ and $\chi \rightarrow c b s$ decay times (indistinguishable at the scale of the figure) obtained in [37]. . . . . . . . . . . .

2.4 The $\chi$ decay in the MFV mediator model through the off-shell scalar mediators $\phi_{L, R}, \varphi_{L}$ (left), and through the off-shell fermion $\psi$ and scalar $\phi$ mediators in the FN model (right). . . . . . . . . . . . . .

2.5 Box diagrams contributing to the neutral meson mixing. In the MFV model, there is also a contribution with both $\phi_{L}$ and $\varphi_{L}$ in the loop, while $\phi_{R}$ contributions are suppressed and can be ignored. . . . . . . . . .

2.6 Meson mixing constraints on the couplings $\kappa_{1,2}$ in the MFV mediator model (left) and $g_{q, d}$ in the FN model (right), taking $m_{\phi_{L}}=m_{\varphi_{L}}=500$ $\mathrm{GeV}$ and $m_{\phi}=200 \mathrm{GeV}, m_{\psi}=20 \mathrm{GeV}$ respectively. The excluded regions lie above and to the right of the curves. . . . . . . . . . . 
2.7 The $g g \rightarrow \phi \phi^{\dagger}$ (solid blue), $q q \rightarrow \phi \phi^{\dagger}$ (dot-dashed red) and $g q \rightarrow \phi j$ (solid light blue) contributions to the pair-production and single-production cross-section at the LHC with $\sqrt{s}=14 \mathrm{TeV}$ as a function of a mass of a color triplet scalar $\phi$, a mediator in the FN model. . . . . . . . . . . .

2.8 Constraints on the scalar mediator $\phi$ in the FN model, and $\phi_{L}, \varphi_{L}, \phi_{R}$ in the MFV model that follow from the CMS search for pair-produced dijet-resonances [84]. The states in the same flavor multiplet are taken to be mass-degenerate. . . . . . . . . . . . . . . . . . .

2.9 The 95\% exclusion limit on $\phi \phi^{\dagger}$ production in the FN model for the $b \bar{b} \psi \bar{\psi}$ final state, where $\psi$ escapes the detector and sbottom search applies [85]. The solid blue (dashed red) line is for $\phi \rightarrow b \psi$ branching ratios of 50\% and $100 \% \ldots \ldots \ldots \ldots \ldots \ldots \ldots$

3.1 Radiative corrections due to FGBs, $A_{Q}, A_{u}, A_{D}$, split the DM multiplet $\chi$.

3.2 Typical radiative splitting of the fermionic DM multiplet with $\Delta m_{31}\left(\Delta m_{32}\right)$ shown in red (blue) as a function of $g_{U}$, while all other parameters are kept fixed at $g_{Q}=0.4, g_{D}=0.5, M_{u}=600 \mathrm{GeV}, M_{d}=400 \mathrm{GeV}, \lambda_{u}=1$, $\lambda_{u}^{\prime}=0.5, \lambda_{d}=0.25, \lambda_{d}^{\prime}=0.3 . \ldots \ldots \ldots \ldots \ldots \ldots$

3.3 The results of the scan for fermionic DM with radiative mass splitting (upper left panel), in the large mass splitting limit (upper right panel) and scalar (lower panel) flavored DM. Constraints from perturbativity (grey), $t^{\prime}$ (dark magenta) and dijet resonance (orange) searches, flavor bounds (light magenta), early-time cosmology (blue) and direct DM detection (brown) are consecutively applied. Allowed parameter points are denoted by green. For scalar flavor DM (right) we show the LUX and perturbativity bounds as two grey bands. The four benchmark points for fermionic flavored DM are denoted by a diamond, a triangle, a hexagram and a pentagram. . . . . . . . . . . . .

3.4 The ratio of masses of the next-to-lightest to the lightest FGBs, $m_{A^{23}} / m_{A^{24}}$ for radiatively split DM multiplet (upper left panel), and for the large mass splitting limit (upper right panel), as functions of the DM mass, $m_{\chi_{1}}$, for the fermionic flavored DM. Lower panel shows the relative radiative mass splitting in the DM multiplet. The constraints due to perturbativity (grey), too large relic abundance (light blue), early cosmology (dark blue), flavor and direct bounds (dark red), are applied consecutively, leaving allowed points (green). . . . . . . . . . . . . 
3.5 The maximal decay time of the two heavy states in the DM multiplet as functions of DM mass (left) and the minimal mass splitting in the DM multiplet (right) for radiatively split fermionic flavored DM. The color coding is as in Fig. 3.4. . . . . . . . . . . . . . . . .

3.6 The Feynman diagrams for the dominant processes in the DM annihilation for fermionic (left) and scalar (right) flavored DM. For scalar DM only one representative diagram is shown; other relevant final states include $b \bar{b}, c \bar{c}, \tau \tau$ and $t \bar{t}, h h, Z Z$ (when kinematically allowed). . . . . . . 54

3.7 The Higgs-DM coupling, $\lambda_{H}$, as a function of DM mass that gives the correct relic abundance for the Higgs portal scalar DM (red band). The upper (lower) dashed edge corresponds to the limit where $\phi_{2,3}$ decay much after (before) the thermal freeze-out of $\phi_{1}$. The LUX bound, assuming correct relic abundance, is shown as a shaded grey region. . . .

3.8 The predicted spin-independent cross section for DM scattering on nuclei as a function of DM mass for radiatively split fermionic DM (left) and in the large mass-splitting limit (right). The LUX bound is the brown shaded region. The color coding for the points is as in Fig. 3.3. . . . . . 63

3.9 The dijet production cross section at $8 \mathrm{TeV}$ LHC as a function of the lightest FGB mass for radiatively split fermionic DM (left) and in the large mass splitting limit (right). The 95\% CL limit from Ref. [133] is denoted with a solid orange line. The color coding is the same as in Fig. 3.4. . . .

3.10 Mass spectrum and flavor decomposition (upper panel), DM relic density as a function of the DM mass with all other parameters fixed (lower left panel) and the pattern of effects in selected flavor observables (lower right panel) for the fermionic flavored DM benchmark 1 . The input benchmark-point parameters are listed in the center. See text for details. 71

3.11 Same as Fig. 3.10 for benchmark 2. . . . . . . . . . . . . . . 73

3.12 Same as Fig. 3.10 for benchmark 3. . . . . . . . . . . . . . . 74

3.13 Same as Fig. 3.10 for benchmark 4. . . . . . . . . . . . . 75

4.1 DM annihilation channels in the Higgs-portal models. . . . . . . . . . 85 
4.2 Bounds from LUX (blue band), XENON100 (green band) and the invisible Higgs decay width (black dashed line and grey region) on the Higgs-portal coupling $g_{\chi}$ for scalar DM, assuming vanishing (left) and SM (right) Yukawa couplings to $u, d, s$ quarks. The red line denotes $g_{\chi}$ as a function of DM mass, $m_{\chi}$, for which the correct relic abundance is obtained, while $g_{\chi}$ in the yellow region leads to non-perturbatively large Higgs decay width, $\Gamma_{h}>m_{h}$, and is excluded. Constraints from Fermi-LAT searches for DM annihilation in dwarf spheroidal galaxies are denoted by the orange band. . . . . . . . . . . . .

4.3 Bounds on the Higgs-portal coupling $g_{\chi}$ for scalar DM, assuming maximal allowed values for the Yukawa couplings to the $u, d, s$ quarks (left to right), keeping all the other couplings to their SM values. The color coding is the same as in Fig. 4.2. . . . . . . . . . . . . . .

4.4 Bounds on the Higgs-portal coupling for vector DM, assuming vanishing (left) and SM (right) Yukawa couplings to $u, d, s$ quarks. The color coding is the same as in Fig. 4.2. . . . . . . . . . . . . . . .

4.5 Bounds on the Higgs-portal coupling for vector DM, assuming maximal allowed values for the Yukawa couplings to the $u, d, s$ quarks (left to right), keeping all the other couplings to their SM values. The color coding is the same as in Fig. 4.2. . . . . . . . . . . . . . .

4.6 Bounds on the Higgs-portal coupling for Dirac DM, assuming $\Lambda=1 \mathrm{TeV}$ and vanishing (left) and SM (right) Yukawa couplings to $u, d, s$ quarks. The color coding is the same as in Fig. 4.2. . . . . . . . . . . .

4.7 Bounds on the Higgs-portal coupling for Dirac DM, assuming $\Lambda=1$ $\mathrm{TeV}$ and maximal allowed values for the Yukawa couplings to the $u, d, s$ quarks (left to right), keeping all the other couplings to their SM values. The color coding is the same as in Fig. 4.2. . . . . . . . . . . . .

4.8 Bound on the pseudoscalar Higgs-portal coupling for Dirac DM, assuming $\Lambda=1 \mathrm{TeV}$ and vanishing (left) and SM (right) Yukawa couplings to $u, d, s$ quarks. The color coding is the same as in Fig. 4.2. . . . . . . . .

4.9 Bounds on the pseudoscalar Higgs-portal coupling for Dirac DM, assuming $\Lambda=1 \mathrm{TeV}$ and maximal allowed values for the Yukawa couplings to the $u, d, s$ quarks (left to right), keeping all the other couplings to their SM values. The color coding is the same as in Fig. 4.2. . . . . . . 
4.10 The effect of large light-quark Yukawa couplings on the indirect detection bounds from Fermi-LAT observations of Milky Way dwarf spheroidal satellite galaxies $[130] . \ldots \ldots \ldots \ldots \ldots$

4.11 Left: The ratio of direct detection bounds on $g_{\chi}$ from Xenon target varying $\kappa_{u}$ (dark red), $\kappa_{d}$ (light red), or $\kappa_{s}$ (blue), and the bound on $g_{\chi}$ assuming SM Higgs Yukawa couplings. The LHC upper bounds on $\kappa_{i}$ are denoted by vertical dashed lines with shaded regions excluded. Right: the ratio of predicted scattering cross sections. The dotted lines correspond to negative values of $\kappa_{q} . \ldots \ldots \ldots \ldots$

4.12 The $\gamma$-ray excess in the recently discovered dwarf spheroidal galaxy Reticulum 2, interpreted as a signal of DM annihilating into $b \bar{b}$ pairs, is shown as the black $1 \sigma$ contour (see Ref. [231] for details). The orange lines show the 95\% CL exclusion limits at the 14-TeV LHC (solid line) and a prospective 100-TeV hadron collider (dashed line), obtained by rescaling the bounds given in Ref. [156]. The remaining color coding is the same as in Fig. 4.2. See text for more details. . . . . . . . . . . . . . 115

5.1 An illustration of an example of a CPV sensitive observable in $h \rightarrow$ $\gamma \gamma \rightarrow 4 e$. The Higgs decays to on-shell photons which convert in the detector. The distribution of the azimuthal angle $\varphi$ between the two planes formed by each positron and its parent photon depends on the Higgs couplings to $\mathrm{CP}$ even and odd operators. The electrons do not need to be co-planar with the corresponding photon-positron planes. The positron-photon plane is shown in magenta and the electron-photon plane in blue. For further details and subtleties see the main text. . . . . 121

5.2 A linear polarization thought experiment in Higgs rest frame. $\mathcal{P}_{1,2}$ are linear polarizers oriented orthogonal to the photon momentum direction. The angle $\phi$ is measured between the linear polarization vectors

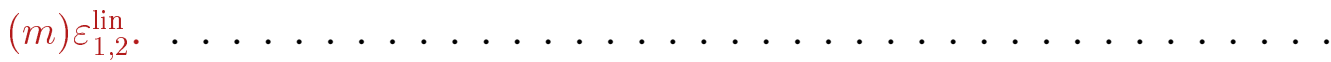

5.3 The contributions to photon cross-section on ${ }^{28} \mathrm{Si}, \sigma_{\gamma}\left({ }^{28} \mathrm{Si}\right)$, from $\mathrm{BH} e e^{+} e^{-}$ pair production in nuclear field (solid blue line), pair production due to scattering on electron cloud (red dashed), Compton scattering (dotdashed yellow) and Rayleigh scattering (magenta double dot-dashed), as a function of photon energy $E_{\gamma}$. Calculated using NIST's XCOM database [271]. 
5.4 Cumulative distributions of Bethe-Heitler conversion cross-section for a photon with $E_{\gamma}=60[\mathrm{GeV}]$ scattering on a ${ }^{28} \mathrm{Si}$ nucleus, with respect to various opening angle cuts. Three distributions are shown: $P\left(\theta_{\ell \ell}>\theta_{\text {cut }}\right)$, i.e. with photon-lepton opening angles, $\theta_{ \pm}$, unconstrained (blue line); $P\left(\theta_{+}\right.$and $\left.\theta_{\ell \ell}>\theta_{\text {cut }}\right)$ with electron-photon opening angle $\theta_{-}$ unconstrained (red dashed line); $P\left(\theta_{ \pm}\right.$and $\left.\theta_{\ell \ell}>\theta_{\text {cut }}\right)$ (black dot-dashed

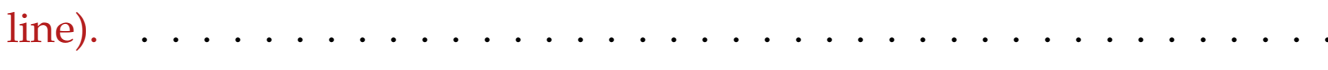

5.5 The elastic form factor $G_{2}^{\text {el }}\left(q^{2}\right)$. The dashed lines show the limiting behavior $G_{2}^{\text {eq }} \sim a^{4} q^{4}$ for $\left|a^{2} q^{2}\right| \ll 1$ (green dashed line) and $G_{2}^{\mathrm{el}} \sim 1$ for $\left|a^{2} q^{2}\right| \gg 1$ (red dashed line). The scale at which screening of the nucleus becomes important is denoted by $a$, which is smaller than the Si atomic radius, $r_{\text {atom. }}$. At scales well outside the atom, corresponding to small $-q^{2}$, nuclear conversion is suppressed by the form factor screening. . .

5.6 Definitions of local spherical polar angles $\left\{\theta_{i_{ \pm}}, \phi_{i_{ \pm}}\right\}$. Note in particular that azimuthal angles, $\phi_{i_{ \pm}}$are positively oriented with respect to their parent photons, and are defined with respect to an azimuth $(\hat{m}) x$ common to both branches. Polar angles are defined with respect to parent photon momentum (black dotted). . . . . . . . . . . . . . . . . 141

5.7 Top panel: $Z_{B}$ in the $c-\tilde{c}$ plane, with the SM point at $(c, \tilde{c})=(-0.81,0)$. Bottom panel: $Z_{B}^{c}$ as a function of $\xi=\tan ^{-1}(\tilde{c} / c)$. The scatter of the data points is a numerical artifact. . . . . . . . . . . . .

5.8 Left: Illustration of $\mathcal{O}(1)$ oscillations and phase shifts in the HBH differential rate for a sample coplanar kinematic configuration. The azimuthal angle $\varphi$ in this slice is defined as in Eq. (5.46). The kinematic configuration is: $E_{i_{+}}=E_{i_{-}}=m_{h} / 4, \theta_{i_{+}}=10^{-4}, \theta_{i_{-}}=2 \theta_{i_{+}}$so that $\gamma_{ \pm} \theta_{ \pm} \sim 10 \gg 1$ and $\gamma_{+}=\gamma_{-}$, cf. analysis of Eq. (5.51). Right: The azimuthal distribution $d \Gamma / d \varphi$ for $\xi=0$ and for $\xi=\pi / 4$ with a polar angle cut $\theta_{i_{ \pm}}>10^{-5}$ and $\theta_{\ell \ell}>10^{-5}$. The modulation amplitude is $2 \%$, but will grow to $\mathcal{O}(1)$ once optimization cuts are applied, see Sec. 5.5.4. . . . . . . . . . . . . 149 
5.9 The differential HBH rate $d \Gamma / d \varphi$ as a function of the azimuthal angle $\varphi$ between the positrons setting the CPV parameter to $\xi=0$ (blue histograms) and $\xi=\pi / 4$ (red histograms). The histograms are binned Monte Carlo events with different cuts on parameters $\mathcal{T}_{n}$, Eq. (5.52), as denoted in the panels. The solid blue (red dot-dashed) curves are the result of fitting the normalized binned events to Eq. (5.50) for the $\xi=0$ $(\xi=\pi / 4)$ cases with $\xi$ also floated in the fit. The angular resolution cut

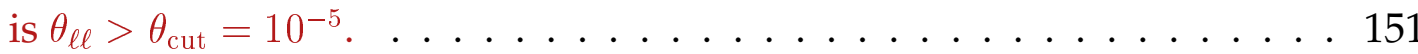

5.10 The same as in Fig. 5.9, but with the opening angle cut $\theta_{\ell \ell}>\theta_{\text {cut }}=10^{-4}$.

5.11 Top panels: The azimuthal distributions $d \Gamma / d \varphi$ for $\xi=0$ (grey histograms) and for $\xi=\pi / 20, \pi / 4$ (blue histograms, left and right top panels) with $\mathcal{S}_{1,2}>1$ on the domain $\delta_{1,2} \in[3 \pi / 4,5 \pi / 4]$. The solid (dashed) curves denote fits to Eq. (5.48), with $\xi$ a free parameter in the fits. The bottom left (right) panel shows the acoplanarity distributions, $d \Gamma / d \delta \phi$, $\delta \phi \equiv\left(\phi_{+}-\phi_{-}\right) \bmod 2 \pi$ for each photon branch, displayed by blue and gray histograms respectively, with $\xi=\pi / 4$ and no $\mathcal{S}$ cut $\left(\mathcal{S}_{1,2}>1\right)$. Note that the scale varies between these two plots. The corresponding cumulative acoplanarity distributions $\operatorname{cdf}(\delta \phi)$ for each branch are denoted by solid black and grey dashed lines on each plot. In all panels the angular resolution cut (5.38) of $\theta_{\text {cut }}=10^{-5}$ was applied. . . . . . . . . . . 154

5.12 Comparison of different sensitivity parameter cut schemes, in terms of cut efficiency, for various angular resolution cuts. The black data point on each plot denotes the efficiency and $\langle\mathcal{B}\rangle /\langle\mathcal{A}\rangle$ for its angular resolution cut alone, with no enhancements from sensitivity parameter cuts. . . . . 156

6.1 Kinematic configuration and coordinate choices. $B$ momentum is denoted by $p_{B}$, and azimuthal angles are defined with respect to the $K^{*}-\gamma$ decay plane (blue). This plane contains $(m) p$ and $(m) k((m) P$ and $(m) k)$ in the lab frame ( $K^{*}$ or $B$ rest frames); momenta lying in this plane in each frame are shown in gray. Left: Lepton polar angles $\theta_{ \pm}$and azimuthal angles $\phi_{ \pm}$in the lab frame. Middle: $\theta_{K}$ and $\phi_{K}$ polar angles in the $K^{*}$ rest frame. Right: The photon polar angle, $\theta_{\gamma}$, in the $B$ rest frame. 166 
6.2 Left: The fit value for $\mathcal{C}$ with the $\pm 1 \sigma$ error band as a function of the polar angle cuts $\theta_{\ell \ell, \pm}>\theta_{\mathrm{c}}$ (see Fig. 6.1). The peak value of $\mathcal{C}$ approximately coincides with the peak of the $\theta_{ \pm}$marginal distribution (see the left panel of Fig. D.2). Right: Normalized differential distribution $d \mathcal{R} / d \psi$ for four different $(r, \phi+\delta)$ couplets and $\theta_{\mathrm{c}}=10^{-6}$. Also shown are theory predictions (gray) for the input values of $(r, \phi+\delta)$ and the extracted value $\mathcal{C}\left[\theta_{c}=10^{-6}\right]$ in eq. $(6.41) \ldots \ldots \ldots \ldots \ldots \ldots \ldots$

6.3 Upper panels: The coefficient $\mathcal{C}$ as a function of the cut efficiency for the $\mathcal{S}$ (blue) and $\mathcal{T}$ (gold) kinematic cuts, with polar cuts $\theta_{\mathrm{c}}=10^{-6}$ (left) and $\theta_{\mathrm{c}}=5 \times 10^{-4}$ (right). The colored regions depict the $\pm 2 \sigma$ statistical error bands. The equivalent effective statistics curve $\Sigma=1$, i.e. $\mathcal{C}=\mathcal{C}_{0} / \sqrt{N / N_{0}}$, is also shown (gray). Lower panels: Statistics enhancement $\Sigma$ as a function of the cut efficiency. The maxima correspond to the optimum cuts $\mathcal{S}>\mathcal{S}_{\mathrm{c}}^{\mathrm{opt}}$ and $\mathcal{T}>\mathcal{T}_{\mathrm{c}}^{\mathrm{opt}}$ (colored dots in all panels). . . . .

6.4 The enhancement in $\mathcal{C}$ as a function of $\mathcal{S}$. The secondary $y$-axis shows the corresponding cut efficiency. The colored regions depict the $\pm 2 \sigma$ statistical error bands. . . . . . . . . . . . . . . . . .

A.1 Example Feynman diagrams for the decay of $B=2$ DM. The diagram on the left shows the tree level decay whereas the one on the right shows the loop-induced decay. . . . . . . . . . . . . . . . . . . .

B.1 The FGB contributions to $V-A$ current operator in the effective weak Hamiltonian. Left panel shows the values of the complex ratio $\delta C_{1}^{b d} / \delta C_{1}^{b s}$ for our scan points, with green points satisfying all constraints, magenta points excluded by flavor constraints and grey points by perturbativity considerations. The point $\delta C_{1}^{b d} / \delta C_{1}^{b s}=\left(V_{t d}^{*} / V_{t s}^{*}\right)^{2}$, obtained if the MFV operator with the smallest number of Yukawa insertions dominates, is denoted by a cross. The right panel shows the cumulative func-

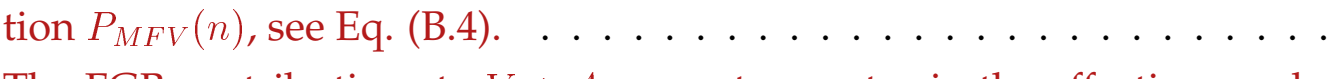
198

B.2 The FGB contributions to $V+A$ current operator in the effective weak Hamiltonian. Left panel shows the complex ratio $\delta \tilde{C}_{1}^{b d} / \delta \tilde{C}_{1}^{b s}$ for our scan points with the same color coding as in Fig. B.1. The point $\delta \tilde{C}_{1}^{b d} / \delta \tilde{C}_{1}^{b s}=$ $\left(m_{d} / m_{s}\right)^{2}\left(V_{t d}^{*} / V_{t s}^{*}\right)^{2}$, obtained if the MFV operator with the smallest number of Yukawa insertions dominates, is denoted by a cross. The right panel shows the cumulative function $\tilde{P}_{\mathrm{MFV}}(n)$, see Eq. (B.6). . . . . . . . 200 
B.3 The fraction of benchmarks as a function of the off-diagonal couplings of the heaviest and next-to-heaviest DM components to the lightest FGB $\left(A^{24}\right)$ normalized by the diagonal coupling of the heaviest component. . 202

C.1 Spectrum of the positron energy $\mathcal{E}_{+}=E_{+} / E_{\gamma}$. No opening angle cut was applied in the left hand figure and an opening angle cut of $10^{-4}$ was applied in the right hand one. The histograms were created with MC events and the solid curves are results of numerically integrating the differential cross section. The dashed curve in both figures is the result of numerically integrating the differential cross section over the entire range of $\delta \phi$ as opposed over the range $[0.6 \pi, 1.4 \pi] \ldots \ldots \ldots \ldots \ldots$

C.2 Polar angle distribution of the leptons. No opening angle cut was applied in the left hand figure and an opening angle cut of $10^{-4}$ was applied in the right hand one. The histograms were created with MC events and the solid curves are results of numerically integrating the differential rate expression. The small bump in the right hand figure $\left(\sim 10^{-4}\right)$ is a result of applying an opening angle cut. Its location is a function of the cut. . . . . . . . . . . . . . . . 216

C.3 Left: Distribution of the azimuthal angle between the leptons. The histograms were created with MC events and the solid curves are results of numerically integrating the differential rate expression. Right: The cumulative distribution function of the relative azimuthal orientation, $P(\delta \phi / \pi \in[1-\Delta, 1+\Delta])$, from numerical integration. . . . . . . . 217

D.1 Left: The cumulative distribution function (CDF) of the opening angle between the leptons. Right: The normalized distribution of the photon energy in units of the $B$ mass for two different $\theta_{\ell \ell}$ cuts. . . . . . . . 225

D.2 Left: The normalized polar angle distribution of the positron for two different values of the opening angle cut $\theta_{\ell \ell}$. Right: the positron energy as a fraction of the photon energy for two values of $\theta_{\ell \ell}$. The distribution exhibits the expected behavior for $\mathrm{BH}$ conversion. It is symmetric about $1 / 2$ and prefers that one lepton carry a larger fraction of the photon energy.226 


\section{List of Tables}

2.1 Leading decay modes for the $B=1,2,3$ ADM assuming MFV or FN flavor breaking. The dimensionality of the decaying operators are denoted in the 2nd column. With the suppression scales $\Lambda$ given in the 6th and 9th column the ADM decay time is $\tau \simeq 10^{26} \mathrm{~s}$. The $B=3$ ADM decays to quarks are kinematically forbidden. . . . . . . . . . . . . .

2.2 The gauge and global charge assignment for the three scalar mediators, $\phi_{L}, \varphi_{L}$ and $\phi_{R}$, in the first UV completion toy model for which we assume the MFV flavor breaking pattern. . . . . . . . . . . .

2.3 Gauge and $B-L$ charges of the mediators $\phi$ and $\psi$ in the second UV completion toy model. We also assume the FN flavor breaking pattern. . 24

2.4 The $95 \%$ C.L. bounds on the MFV and FN mediator models from meson mixing. Taking $m_{\phi_{L}}=m_{\varphi_{L}}=m_{\phi}=1 \mathrm{TeV}$ and $\kappa_{1}=\kappa_{2}\left(g_{q}=g_{d}\right)$ gives the upper bounds on the couplings in the 2nd(4th) column. Taking in turn $\kappa_{1,2}=g_{q, d}=1$ gives lower bounds on the mediator masses in the 3rd and 5th columns. The mass of the fermion in the FN model is fixed to $m_{\psi}=20 \mathrm{GeV}$ (see Sec. 2.6.3). The bounds are not very sensitive to $m_{\psi}$. .

4.1 Predictions for the flavor diagonal up-type Yukawa couplings in a number of new physics models (see text for details). . . . . . . . . . . . . 102

4.2 Predictions for the flavor diagonal down-type Yukawa couplings in a number of new physics models (see text for details). . . . . . . . . . . 103

4.3 Predictions for the flavor violating up-type Yukawa couplings in a number of new physics models (see text for details). In the SM, NFC and the tree-level MSSM the Higgs Yukawa couplings are flavor diagonal. The estimates of the $C P$-violating versions of the flavor-changing transitions, $\kappa_{i j} / \kappa_{t}$, are the same as the $C P$-conserving ones, apart from substituting "Im" for "Re" in the "MFV" row. . . . . . . . . . . . . . . 104 
4.4 Predictions for the flavor violating down-type Yukawa couplings in a number of new physics models (see text for details). In SM, NFC and tree level MSSM the Higgs Yukawa couplings are flavor diagonal. The estimates of the $C P$-violating versions of the flavor-changing transitions, $\kappa_{i j} / \kappa_{b}$, are the same as the $C P$-conserving ones, apart from substituting "Im" for "Re" in the "MFV" row. . . . . . . . . . . . . . . . . . . . . 104

5.1 Expected number of events after the application of $\mathcal{S}$ or $\mathcal{T}$ cuts with $\theta_{\ell \ell}>10^{-4}$ to obtain $\langle\mathcal{B}\rangle /\langle\mathcal{A}\rangle \sim 20 \%$. The Higgs production cross section includes the gluon fusion and VBF channels only and is taken from [287]. 155

6.1 Extracted values of $r$ and $(\phi+\delta)$ from an MC sample with input values $(0.2, \pi / 4) \ldots \ldots \ldots \ldots \ldots \ldots 18 \ldots \ldots \ldots$

A.1 Partial decay widths, $\Gamma_{i}$, and related decay times, $\tau_{i}=1 / \Gamma_{i}$, for representative decay channels above kinematical thresholds (1st column) assuming the MFV flavor breaking ansatz. The EFT scale is set to $\Lambda=1$ $\mathrm{TeV}$. The last column denotes whether the dominant amplitude is tree level or 1-loop, while the 2nd and the 3rd columns give the decay vertex transition and the partonic transition after the potential $W$ exchange, respectively. . . . . . . . . . . . . . . . . . 196

C.1 The details on the MC generation of $\mathrm{BH}$ events, with phase space variables (1st column) for $\mathrm{C}(\mathrm{C}++/$ Java) generated in the range given in the 2nd (4th) column according to the distribution given in the 3rd (5th) column (for details see text). . . . . . . . . . . . . . . . . . 220 


\section{Chapter 1}

\section{Introduction}

It is worth emphasizing a remarkable fact: we understand the physics of our Universe on distance scales that span 40 orders of magnitude. This understanding has been distilled through the scientific process into two phenomenological theories: the standard model of particle physics (SM), and the cold dark matter model with the addition of a cosmological constant $(\Lambda \mathrm{CDM})$. The former describes the interactions of the fundamental particles while the latter describes the evolution and dynamics of our Universe on the largest scales.

Thus far, all experimental tests of the SM are in good agreement with its predictions. There are, nonetheless, tantalizing experimental "anomalies" that are in tension with the SM predcitions. At the time of this writing the anomalies included: the muon $g-2$ anomaly $(3.6 \sigma)$, the $B$ meson anomaly in $B^{0} \rightarrow K^{*} \mu^{+} \mu^{-}(2.9 \sigma)^{1}$, and a putative signal of flavor violation in the Higgs decay $h \rightarrow \mu \tau(2.6 \sigma)$ to name just a few. Such deviations could be signals of new physics beyond the SM (BSM). Moreover, the SM leaves some unanswered questions which include

- The origin of neutrino masses

\footnotetext{
${ }^{1}$ Taking the [4-6] GeV ${ }^{2}$ bin only.
} 
- The hierarchy problem

- Dark matter

- The flavor puzzle

- The origin of the matter/anti-matter asymmetry

The answer to some of these questions could, and in some cases must, lie in BSM physics.

In this thesis, we will focus on BSM physics related to DM and CP violation (CPV) which is related to the matter/anti-matter asymmetry. In particular, we will explore two DM model building scenarios where the DM stability derives from flavor symmetries. Then, on a more speculative note, we will exploit the Higgs portal DM model to constrain the light Yukawa couplings. Finally, we will develop a technique to probe CPV in Higgs couplings and show that this technique could also be useful to probe BSM physics effects in radiative rare $B$ meson decays.

\section{Dark matter stability and the SM flavor structure}

The SM Lagrangian $\left(\mathscr{L}_{\mathrm{SM}}\right)$ possesses an enhanced $U(3)^{5}$ symmetry in the high energy limit where all the fermion masses are negligible in comparison with the energy scale $\left(m_{f} \rightarrow 0\right)$. Focusing on the quark sector, the subgroup

$$
\mathcal{G}_{F} \equiv S U(3)_{Q} \otimes S U(3)_{U} \otimes S U(3)_{D} \subset U(3)^{3}
$$

is only broken by the SM Yukawa interactions. However, $\mathscr{L}_{\text {SM }}$ can be made formally invariant under $\mathcal{G}_{F}$ if the Yukawa matrices are promoted to spurions (spurious field degrees of freedom) which transform non-trivially under $\mathcal{G}_{F}$. This is a useful way to parametrize the breaking of the flavor symmetry $\mathcal{G}_{F}$ in BSM models. Further, asserting 
that the only source of flavor breaking is from the SM Yukawas goes by the name of minimal flavor violation (MFV).

As we will see in chapter 2, invoking the MFV hypothesis in a BSM model leads to additional suppression in the Wilson coefficient of the effective operators. This suppression will afford us a metastable $\mathrm{DM}$ candidate with $\mathrm{TeV}$ scale mediators. The $\mathrm{TeV}$ scale is significant for two reasons. Firstly, because $\mathrm{TeV}$ scale mediators are accessible at the large hadron collider (LHC) and hence allow us to discover or exclude the proposed toy models. Second, solutions to the hierarchy problem which was briefly mentioned above typically require new physics at the $\mathrm{TeV}$ scale to cancel the quadratic divergence of the Higgs mass.

If $\mathcal{G}_{F}$ is broken by spurions that have zero triality ${ }^{2}$, there is a discrete $Z_{3}$ subgroup that is left unbroken. This is useful because there exists a subgroup of the direct product $Z_{3} \otimes Z_{3}^{c}$ which we call $Z_{3}^{\chi}$ that is consequently left unbroken ${ }^{3}$. Not only that, but all SM fields are singlets under $Z_{3}^{\chi}$. Thus, the lightest state that carries a charge under $Z_{3}^{\chi}$ is automatically stable and could therefore be a DM candidate. We will explore the phenomenology (DM and flavor) in a flavor model where $\mathcal{G}_{F}$ is fully gauged.

\section{Matter/anti-matter asymmetry and CPV in the SM}

There is observational evidence that the Universe is made up entirely of matter and not antimatter.Antimatter appears to be predominantly a secondary by-product of particle collisions, e.g. at particle accelerators or between cosmic rays and the interstellar medium (ISM) or the earth's atmosphere. There are three required conditions in order for the Universe to evolve a net baryon asymmetry - the Sakharov conditions [3]: i) Baryon number violation, ii) $\mathrm{C}$ and $\mathrm{CP}$ violation, and iii) departures from thermal equi-

\footnotetext{
${ }^{2}$ See, e.g., [1] for a definition of triality for $S U(3)$ tensors and [2] for the definition of flavor triality.

${ }^{3}$ Here, $Z_{3}^{c}$ is the center group of the QCD gauge group $S U(3)_{c}$.
} 
librium. Here, $\mathrm{C}$ is charge conjugation and $\mathrm{CP}$ is charge conjugation combined with a parity transformation. The SM does provide all the necessary ingredients. However, the parameters of the SM are not sufficient for successful baryogenesis. For instance, the only $\mathrm{CP}$ violating parameter in the $\mathrm{SM}$ is the phase of the CKM matrix and it is not sufficient to produce the observed asymmetry. On the other hand, the departure from local thermal equilibrium condition could be fulfilled by the phase transition between the broken and unbroken phases of the electroweak gauge group. Unfortunately, the parameters of the Higgs potential (the cubic and quartic couplings) only allow for weak first order phase transition which is insufficient to satisfy the third condition Sakharov condition.

Another possibility for BSM sources of CP violation (CPV) could be in the Higgs sector. Since its discovery, the experimental constraints on the couplings of the Higgs boson to the SM gauge bosons and third generation fermions have become statistically significant. This does not preclude couplings between the Higgs boson and new heavy fermions for example. In general, such couplings could be $\mathrm{CP}$ violating. If these fermions are too heavy to be probed directly at the LHC, they could still be discovered via quantum effects. For example, if they are colored, they would contribute to gluon fusion which is the main production mode of the Higgs at the LHC. Further, if they are electrically charged, they would contribute to the $h \rightarrow \gamma \gamma$ process which is one of the cleanest signals of the Higgs due to low backgrounds. This possibility is investigated in detail in chapter 5 where we develop observables sensitive to CPV in the Higgs coupling to photons.

\section{Organization}

The thesis is organized as follows. In chapter 2, we investigate a model of asymmetric dark matter where the DM is neutral under the SM flavor group but its couplings 
are proportional to flavor breaking. The DM in this case is metastable and its lifetime is bounded by indirect searches from $\gamma$-ray telescopes. Flavor suppression, however, allows the EFT scale to be at the $\mathrm{TeV}$ and thus have interesting signatures at the LHC. In chapter 3, we turn our attention to flavored DM that is charged under the SM flavor group. Here, the DM is absolutely stable due to a remnant $Z_{3}$ subgroup that survives flavor breaking. We then discuss the phenomenology of the DM and the mediators in the context of a non-MFV gauged flavor model. In chapter 4, we discuss the possibility of constraining the light-quark Yukawa couplings in the case of a Higgs portal DM. While current and future colliders are not sensitive to these small Yukawas, DM direct detection rates are. By exploiting the complementarity between different searches, we point out that if Higgs portal DM is discovered, we would obtain a non-trivial constraint to the light Yukawas. In chapter 5, we switch our focus to CPV in Higgs decays to two photons and we develop observables sensitive to CPV. Finally, in chapter 6, we exploit the same technique developed in chapter 5 to extract the photon helicity fraction and weak and strong phases in the $B \rightarrow K^{*} \gamma$ radiative decay. An order $\sim 1$ helicity fraction or weak phase would be a clear signal of new physics. 


\section{Chapter 2}

\section{Continuous Flavor Symmetries and the Stability of Asymmetric Dark Matter}

\subsection{Introduction}

Dark matter (DM) is stable on cosmological time-scales. A principal question about the nature of DM is: what mechanism ensures its stability? Commonly, this is assumed to be a result of an exact symmetry (for a concise review of proposed stabilization mechanisms see, e.g., [4]). One possibility is that the stability of DM is ensured by a gauge symmetry mimicking the way QED gauge invariance ensures the stability of the electron in the standard model [5-7]. A more frequent choice is to introduce a $Z_{2}$ symmetry by hand. A prominent example is $R$-parity in the MSSM which both stabilizes DM and ensures the stability of the proton [8-10]. An exact $Z_{2}$ symmetry can be generated dynamically, e.g., as a remnant of a spontaneously broken $\mathrm{U}(1)$ gauge symmetry, such as $U(1)_{B-L}$ [11-13]. An attractive possibility is that $Z_{2}$, and consequently the DM stability, is an accidental symmetry. Examples include minimal DM [14,15], hidden vector DM [16], and weakly interacting stable pions [17].

In this chapter, we explore a possibility that the discrete $Z_{2}$ that ensures the stability 
of DM is both accidental and approximate. As a result, the DM is metastable with decay times potentially close to the present observational bound of $\tau \gtrsim 10^{26} \mathrm{~s}$. We focus on a particular subset of asymmetric DM models [18] where DM carries baryon number. For recent reviews of asymmetric DM, see $[19,20]$. Our working assumptions are

- Baryon number is a conserved quantum number (it could, for instance, be gauged at high scales).

- There is a sector that efficiently annihilates away the symmetric component. The exact form is not directly relevant for our discussion.

- The observed flavor structure in the quark sector is explained by flavor dynamics in the UV while DM is not charged under flavor.

The flavor dynamics fixes the flavor structure of dark sector couplings to the visible sector in the same way that it fixes the structure of the SM Yukawa interactions. This has two important consequences. First, the exchange of DM in the loops does not generate dangerously large Flavor Changing Neutral Currents (FCNCs). Secondly, and most importantly, a flavor singlet DM is stable on cosmological timescales even for $\mathrm{TeV}$ scale mediators between the dark and visible sectors. In this case, the nature of DM stability can even be probed directly at the LHC.

The underlying flavor symmetry is crucial for the stability of DM. We will demonstrate this for two realizations of flavor physics: the Minimal Flavor Violation (MFV) hypothesis and for abelian horizontal symmetries in the case where DM carries baryon number 2. In this case the mediators leading to the decay of DM can be at $\mathcal{O}(100 \mathrm{GeV})$. In contrast, for completely anarchic flavor couplings where DM couples to all quark flavors with $\mathcal{O}(1)$ couplings, the indirect DM bounds would require the mediators to have masses in the $\mathcal{O}(10 \mathrm{TeV})$ range.

The implications of continuous flavor symmetries for DM interactions have also 
been explored in [2,21-30]. Our analysis differs from these studies in that we are assuming that DM is a flavor singlet (as is the case in most models of DM). This, along with its small mass and conserved baryon number, also ensures that DM is metastable in our setup. The stability of symmetry-less DM in the context of discrete flavor groups has been discussed in [31] (for the potential relation of discrete flavor groups in the leptonic sector and the stability of DM, see also [32-34]). Furthermore, the stability of asymmetric DM due to a mirror baryon number was explored in [35] or due to fractional baryon number in [36]. The decaying DM in the context of ADM models was explored in [37-40].

The chapter is structured as follows. In Sec. 2.2, we review the relation between DM mass and relic abundance in asymmetric DM models. In Sec. 2.3, we give two examples of flavor breaking models at the level of Effective Field Theory (EFT) analysis that can lead to metastable asymmetric DM. In Sec. 2.4, we derive the indirect detection bounds on the two EFT set-ups. In Sec. 2.5, we give two examples of mediators that would lead to the EFT set-ups discussed in Sec. 2.4. The relevant bounds on the mediator masses and couplings, including collider signatures, are derived in Sec. 2.6. Conclusions are given in Sec. 2.7, while appendices contain technical details.

\subsection{Dark matter mass in asymmetric dark matter models}

Asymmetric Dark Matter (ADM) models [18,41-54] address the question of why the DM density, $\Omega_{\chi}$, and the baryon density in the universe, $\Omega_{B}$, are so close to each other, $\Omega_{\chi} \simeq 5.3 \Omega_{\mathrm{B}}$ [55]. In the standard weakly interacting massive particle (WIMP) models of DM this is to some extent pure coincidence. In this case DM is a thermal relic and

$$
\left(\frac{\Omega_{\chi}}{0.265}\right)\left(\frac{h}{0.673}\right)^{2} \sim \frac{3 \times 10^{-27} \mathrm{~cm}^{3} \mathrm{~s}^{-1}}{\langle\sigma v\rangle}
$$


with $\langle\sigma v\rangle$ the thermally averaged DM annihilation cross section. The coincidence $\Omega_{\chi} \sim \Omega_{B}$ then arises due to a fortuitous size of the annihilation cross section for a weakly coupled weak scale DM - the WIMP miracle.

In contrast, in ADM models the observed DM is not a thermal relic. Its relic abundance reflects the asymmetry in DM, $\chi$, and anti-DM, $\chi^{\dagger}$, densities in the early universe. The $\chi$ and $\chi^{\dagger}$ annihilate away, and only the asymmetric component remains. The coincidence of $\Omega_{\chi}$ and $\Omega_{B}$ is then due to the fact that the DM relic abundance has the same origin as the baryon asymmetry. The difference between $\Omega_{\chi}$ and $\Omega_{\mathrm{B}}$ is simply due to the fact that the DM particle is more massive than a proton by a factor of a few. More precisely, to explain the observed $\Omega_{\chi}$ the DM's mass needs to be (see Appendix A.II)

$$
m_{\chi}=N_{0} m_{p} \frac{\Omega_{\chi}}{\Omega_{B}} \frac{1}{(B-L)_{\chi}},
$$

where $m_{p}$ is the proton mass. Here $(B-L)_{\chi}$ is the $B-L$ charge of the $\chi$ field. The exact value of numerical prefactor $N_{0} \simeq \mathcal{O}(1)$ depends on when the operators transferring the baryon asymmetry between the visible and the dark sector decouple. For decoupling temperature above electroweak phase transition, and assuming that there are only the SM fields in the visible sector, gives $N_{0}=1.255$ for DM that is a complex scalar or a Dirac fermion. In this case the required DM mass is

$$
m_{\chi}=(6.2 \pm 0.4) \mathrm{GeV} \frac{1}{(B-L)_{\chi}},
$$

where the error reflects the errors on $\Omega_{\chi}=0.265 \pm 0.011$ and $\Omega_{B}=0.0499 \pm 0.0022[55$, 56]. We thus have

$$
m_{\chi}=\{6.2,3.1,2.1\} \mathrm{GeV}, \quad \text { for } \quad(B-L)_{\chi}=\{1,2,3\} \text {, }
$$

where we only quote the central values. Deviations from the above relations are possi- 


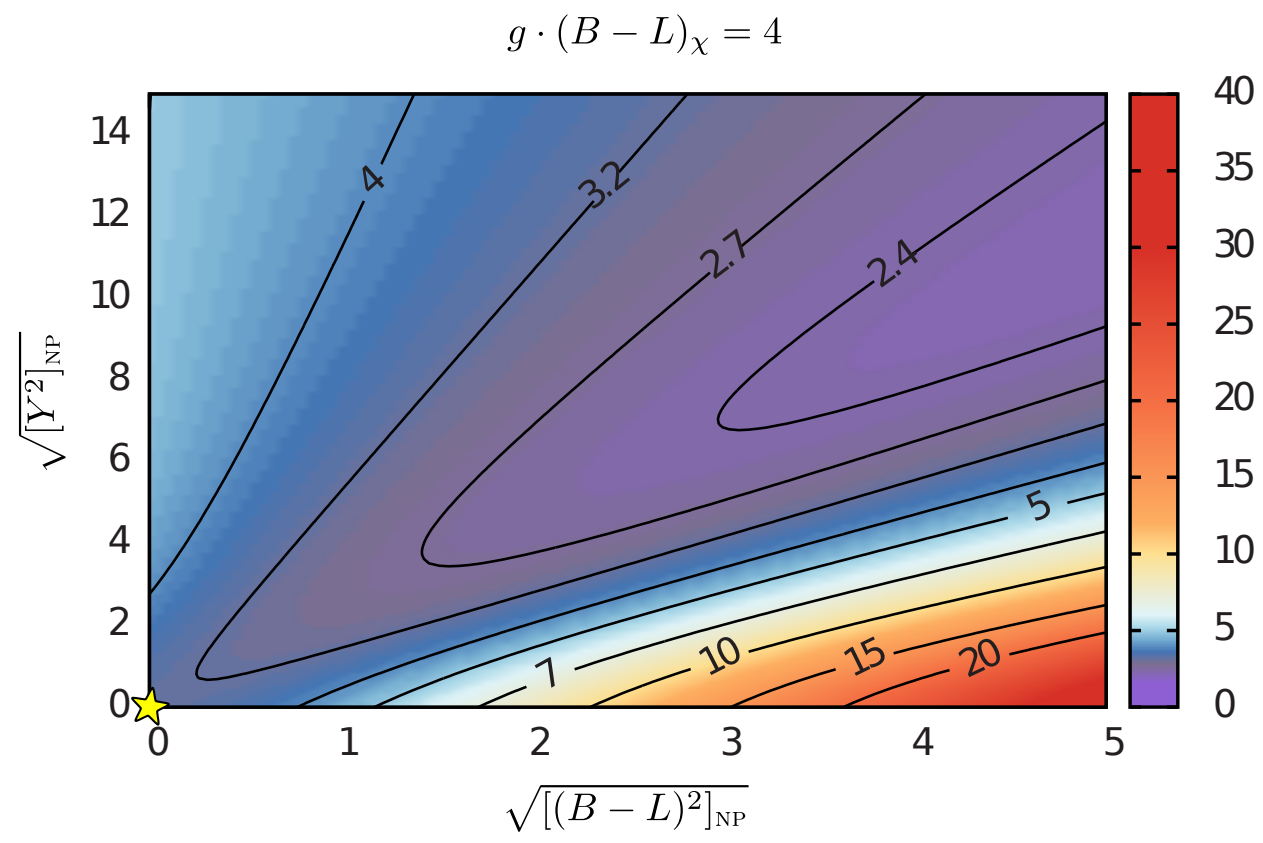

Figure 2.1: Contours of the ADM mass $m_{\chi}$ (in $\mathrm{GeV}$ ) required to obtain the correct DM relic density as a function of $\left[(B-L)^{2}\right]_{N P},\left[Y^{2}\right]_{N P}$, i.e. the $(B-L)^{2}$, and $Y^{2}$ summed over effective d.o.f. in visible NP sector, while keeping $[Y(B-L)]_{N P}=\sqrt{\left[(B-L)^{2}\right]_{\mathrm{NP}}\left[Y^{2}\right]_{N P}}$ and $g(B-L)_{\chi}=4$. The visible sector with only the SM, Eq. (2.2), is denoted by a star.

ble if for instance the visible sector contains additional degrees of freedom beyond the SM. In that case, $m_{\chi}$ in $(2.2)$ is a function of $\left[(B-L)^{2}\right]_{N P},\left[Y^{2}\right]_{N P}$, and $[Y(B-L)]_{N P \text {, }}$ i.e., the $(B-L)^{2}, Y^{2}$ and $Y(B-L)$ summed over effective degrees of freedom in the visible NP sector, cf. Eq. (A.23). The $m_{\chi}$ required to obtain the correct relic abundance is shown in Fig. 2.1. For illustration, we set $[Y(B-L)]_{N P}=\sqrt{\left[(B-L)^{2}\right]_{\mathrm{NP}}\left[Y^{2}\right]_{N P}}$ in the plot and assume that DM is a complex scalar with $(B-L)_{\chi}=2$. We see that for $\left[(B-L)^{2}\right]_{\mathrm{NP}} \sim\left[Y^{2}\right]_{N P} \sim[Y(B-L)]_{N P}$ the deviations from (2.4) are modest, of $\mathcal{O}(1)$. Further deviations from Eqs. (2.2) and (2.4) are possible in more general frameworks such as ADM from leptogenesis [57] or dynamically induced mass mixing [58]. Henceforth, we will assume that $m_{\chi}$ is given by Eqs. (2.2) and (2.4). Our results can be trivially adjusted if this is not the case.

For concreteness we assume in this chapter a thermal history of the universe that 
closely resembles the one in [18] which has several distinct epochs relevant for the ADM relic density. At high temperatures, a $B-L$ asymmetry is generated, e.g., via GUT-like baryogenesis [18] or via leptogenesis [57]. The $B-L$ asymmetry is efficiently transferred between the visible and the DM sectors through asymmetric interactions. We do not require a discrete $Z_{n}$ symmetry in the dark sector so that, unlike [18], the asymmetric interactions can involve just a single $\chi$ field. At low energies, they have a schematic form

$$
\mathcal{O}_{\text {asymm. }} \sim \frac{C}{\Lambda^{6}} \chi(q q)^{3},
$$

taking $(B-L)_{\chi}=2$ complex scalar DM as an example. Here, $C$ is a flavor-dependent coefficient. The asymmetric interactions freeze out at temperature $T_{f} \sim \Lambda \gg m_{\chi}$, below which the $B-L$ asymmetries in the visible and dark sectors are separately conserved. If the flavor breaking is due to a spontaneously broken horizontal symmetry (see Sec. 2.3.2), the freeze out temperature for the above dimension 10 operator in Eq. (2.5) is, using Naive Dimensional Analysis (NDA),

$$
T_{f} \sim\left(1.66 \times \sqrt{g_{*}}\left(16 \pi^{2}\right)^{3} \frac{8 \pi}{C^{2}} \frac{\Lambda^{12}}{m_{\mathrm{PL}}}\right)^{1 / 11} \simeq 480 \mathrm{GeV}
$$

In the numerical evaluation, we used the lower bound $\Lambda=\Lambda_{*}=1.9 \mathrm{TeV}$ from indirect detection Eq. (2.22), taken the effective number of relativistic d.o.f. to be $g_{*}=$ 108.75, corresponding to the SM with a complex scalar DM, and set $C=1$ which is appropriate for the $\chi b \rightarrow b s c t b$ transition dominance (with any permutation of the flavors). Note that $T_{f}$ is above the electroweak phase transition temperature $T_{\text {ew }} \sim 170$ $\mathrm{GeV}$. It is also well below $\Lambda$ so that the use of EFT is justified. If the mediator scale were too low, $\Lambda \lesssim 730 \mathrm{GeV}$ (or $\Lambda \lesssim 400 \mathrm{GeV}$ for MFV breaking), the asymmetric operator would not freeze out before electroweak phase transition started. Consequently, the DM quantum number would not be conserved and the DM density would be washed out. This places a lower bound on the asymmetric mediator masses to be above a few 
hundred GeV.

Finally, at temperatures below DM mass the bulk of the DM efficiently annihilates back to the visible sector through symmetric interactions leaving only the small asymmetric component. We have nothing new to say about this mechanism and refer the reader to a set of model building ideas already present in the literature $[19,59-62]$.

\subsection{Metastability and flavor breaking}

We show next that the DM in ADM models can be stable on cosmological time-scales without invoking discrete $Z_{n}$ symmetries. We assume that the SM quark flavor structure is explained by a continuous flavor group and that the DM carries nonzero baryon number. This is a crucial ingredient in the argument. Since DM is not charged under the flavor group, while the SM fields are, there are no interactions between DM and the SM in the limit that the flavor group is unbroken (all flavor singlet interactions are

forbidden by baryon number conservation). All the interactions between DM and the visible sector thus have to be flavor breaking and this leads to a significant suppression of the DM decay time.

We show this explicitly for two examples of flavor breaking: i) the MFV ansatz, where all the flavor breaking is assumed to be due to the SM Yukawas, and ii) the spontaneously broken horizontal $U(1)$ symmetries. Integrating out the NP fields gives the effective DM decay Lagrangian

$$
\mathcal{L}=\sum_{i} \frac{C_{i}}{\Lambda^{\left(D_{i}-4\right)}} \mathcal{O}_{i}
$$

The sizes of the Wilson coefficients, $\mathcal{C}_{i}$, are fixed by the assumed flavor generating mechanism. We consider the case of DM, $\chi$, that is a SM gauge singlet but carries nonzero baryon number, $B \neq 0$. The lowest dimensional asymmetric local operators 
thus have the generic form

$$
\mathcal{O}_{i}=\chi\left[u^{c}\right]^{n_{u}}\left[d^{c}\right]^{n_{d}}\left[q^{*}\right]^{n_{q}}
$$

where we do not show the contractions of SM gauge indices. Here $\left(n_{u}+n_{d}+n_{q}\right)$ $\bmod 3=0$ since DM is a color singlet. Note that DM needs to carry an integer baryon number in order not to forbid all the asymmetric interactions with the visible sector. Above, $u^{c}, d^{c}$ are the electroweak singlets and $q$ represents the electroweak doublet left-handed quark fields in two component notation, with $q^{*}$ being the corresponding complex conjugated Weyl spinor, see App. A.I. In the down-quark mass basis they are

$$
u^{c} \rightarrow u_{\mathrm{MASS}}^{c}, \quad d^{c} \rightarrow d_{\mathrm{MASS}}^{c}, \quad q=\left(\begin{array}{l}
u \\
d
\end{array}\right) \rightarrow\left(\begin{array}{c}
V_{\mathrm{CKM}} u_{\mathrm{MASS}} \\
d_{\mathrm{MASS}}
\end{array}\right) .
$$

The SM Yukawa matrices are then

$$
Y_{D} \rightarrow Y_{D}^{\text {diag }}, \quad Y_{U} \rightarrow V_{\text {СКм }} Y_{U}^{\text {diag }},
$$

with $Y_{D, U}^{\text {diag }}$ the diagonal Yukawa matrices.

As an example, let us consider fermionic $B=1 \mathrm{DM}$. Two distinct types of operators are allowed

$$
\begin{aligned}
& \mathcal{O}_{1}^{(B=1)}=\left(\chi u^{c}\right)\left(d^{c} d^{c}\right) \rightarrow\left(\chi u_{\mathrm{MASS}}^{c}\right)\left(d_{\mathrm{MASS}}^{c} d_{\mathrm{MASS}}^{c}\right), \\
& \mathcal{O}_{2}^{(B=1)}=\left(\chi q_{\rho}^{*}\right)\left(d^{c} q_{\sigma}^{*}\right) \epsilon^{\rho \sigma} \rightarrow\left(\chi u_{\mathrm{MASS}}^{*} V_{\mathrm{CKM}}\right)\left(d_{\mathrm{MASS}}^{c} d_{\mathrm{MASS}}^{*}\right),
\end{aligned}
$$

where $\rho, \sigma$ are $S U(2)_{L}$ indices while the $S U(3)_{C}$ and flavor indices are implicit and we have chosen one possible Lorentz contraction denoted by the parentheses. 


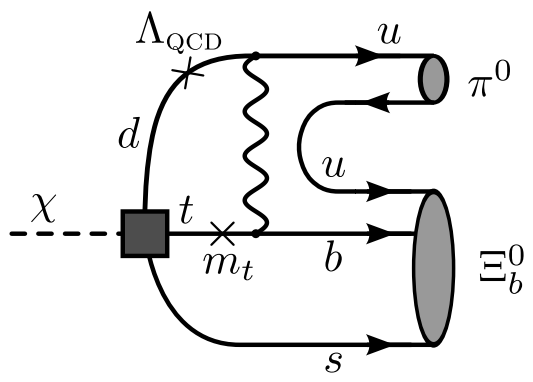

Figure 2.2: Feynman diagram for the decay of DM with $B=1$ assuming MFV. This amplitude leads to the partial decay width $\Gamma_{\chi}^{(1)}$ in Eq. (2.13).

\subsubsection{Minimal Flavor Violation}

The MFV assumption is that, also in the NP sector, the flavor is broken only by the SM Yukawas $Y_{U, D}$ [63-67]. The MFV assumption can be most succinctly cast in the spurion language [64]. In the limit of vanishing quark masses the SM quark sector enjoys an enhanced flavor symmetry $G_{F}=S U(3)_{Q} \times S U(3)_{U} \times S U(3)_{D}$. The Yukawa interactions $u^{c} Y_{U}^{\dagger} q H, d^{c} Y_{D}^{\dagger} q H^{c}$ are formally invariant under $G_{F}$, if $Y_{U, D}$ are promoted to spurions, i.e. if they are assumed to transform under $G_{F}$ as $Y_{U} \rightarrow Y_{U}^{\prime}=U_{Q} Y_{U} U_{U}^{\dagger}$, $Y_{D} \rightarrow Y_{D}^{\prime}=U_{Q} Y_{D} U_{D}^{\dagger}$. Here $U_{Q, U, D}$ are transformations from $S U(3)_{Q, U, D}$, respectively.

This means that the low energy operators in (2.7) also need to be formally $G_{F}$ invariant. Keeping only the minimal insertion of Yukawas, the operators $\mathcal{O}_{1,2}$ in Eq. (2.11) for $B=1 \mathrm{DM}$ are

$$
\begin{gathered}
\mathcal{O}_{1}^{(B=1)}=\left(\chi u_{\alpha}^{c} Y_{U}^{\dagger} Y_{D}\right)_{K}\left(d_{N \beta}^{c} d_{M \gamma}^{c}\right) \epsilon^{K N M} \epsilon^{\alpha \beta \gamma} \\
\rightarrow\left(\chi u_{\mathrm{MASS}}^{c} Y_{U}^{\mathrm{diag} \dagger} V_{\mathrm{CKM}}^{\dagger} Y_{D}^{\mathrm{diag}}\right)_{K \alpha}\left(\left[d_{\mathrm{MASS}}^{c}\right]_{N \beta}\left[d_{\mathrm{MASS}}^{c}\right]_{M \gamma}\right) \epsilon^{K N M} \epsilon^{\alpha \beta \gamma}, \\
\mathcal{O}_{2}^{(B=1)}=\left(\chi q_{K \alpha i}^{*}\right)\left(\left[d_{\beta}^{c} Y_{D}^{\dagger}\right]_{N} q_{M \gamma j}^{*}\right) \epsilon^{i j} \epsilon^{K N M} \epsilon^{\alpha \beta \gamma} \\
\rightarrow\left(\chi u_{\mathrm{MASS}}^{*} V_{\mathrm{CKM}}^{\dagger}\right)_{K \alpha}\left(\left[d_{\mathrm{MASS}}^{c} Y_{D}^{\mathrm{diag} \dagger}\right]_{N \beta}\left[d_{\mathrm{MASS}}^{*}\right]_{M \gamma}\right) \epsilon^{K N M} \epsilon^{\alpha \beta \gamma},
\end{gathered}
$$

where $\alpha, \beta, \gamma$ are the color indices, and $K, N, M$ run over the quark generations.

The two operators lead to the $\chi \rightarrow$ bus decay at the partonic level which is the least 
suppressed kinematically allowed transition. For the operator $\mathcal{O}_{1}$, this transition arises at 1-loop and requires two chirality flips, see Fig. 2.2. The decay amplitude scales as $\sim y_{t} y_{b}$ with an extra loop factor and a chirality flip suppression $\sim m_{t} \Lambda_{\mathrm{QCD}} / m_{W}^{2}$. To be conservative, we count the chirality flip suppression due to the light $u, d, s$ quarks as proportional to $\Lambda_{\mathrm{QCD}}$ and not to the much smaller quark masses. The operator $\mathcal{O}_{2}$ leads to the decay $\chi \rightarrow$ bus at tree level with the decay amplitude suppressed by $\sim y_{b} V_{u b}$. Once the quarks hadronize, the decays appear as $\chi \rightarrow \Xi_{b} \pi$, or $\chi \rightarrow \Lambda_{b} K$, with any number of pions. Using NDA to estimate the decay width gives (setting $V_{t b} \simeq V_{u d} \simeq 1$ )

$$
\begin{aligned}
& \Gamma_{\chi}^{(1)} \sim \frac{\left(y_{t} y_{b}\right)^{2}}{8 \pi}\left(\frac{m_{\chi}}{\Lambda}\right)^{4}\left(\frac{1}{16 \pi^{2}} \frac{m_{t} \Lambda_{\mathrm{QCD}}}{m_{W}^{2}}\right)^{2} \frac{m_{\chi}}{16 \pi^{2}}=6.6 \cdot 10^{-51} \mathrm{GeV}\left(\frac{y_{b}}{0.024}\right)^{2}\left(\frac{4.0 \cdot 10^{6} \mathrm{TeV}}{\Lambda}\right)^{4}, \\
& \Gamma_{\chi}^{(2)} \sim \frac{\left|y_{b} V_{u b}\right|^{2}}{8 \pi}\left(\frac{m_{\chi}}{\Lambda}\right)^{4} \frac{m_{\chi}}{16 \pi^{2}}=6.6 \cdot 10^{-51} \mathrm{GeV}\left(\frac{y_{b}}{0.024}\right)^{2}\left(\frac{4.3 \cdot 10^{7} \mathrm{TeV}}{\Lambda}\right)^{4},
\end{aligned}
$$

for the case where $\mathcal{O}_{1}$ and $\mathcal{O}_{2}$ dominate the decay, respectively. The last $1 / 16 \pi^{2}$ factor is due to three body final state and is required to obtain the correct estimate for the inclusive decay width as can be seen from the optical theorem and the use of the OPE. In the numerics, we use $m_{t}=173 \mathrm{GeV}, m_{\chi}=6.2 \mathrm{GeV},\left|V_{u b}\right|=0.00415$. The numerical prefactor $6.6 \cdot 10^{-51} \mathrm{GeV}=1 /\left(10^{26} s\right)$ is chosen to make contact with the bounds on the DM lifetime from indirect DM searches.

Note that MFV leads to two sources of suppression. First, there is the suppression of the Wilson coefficients due to Yukawa insertions, $y_{b} \sim 0.024$ for $\mathcal{O}_{1}$ and $y_{b} V_{u b} \sim$ $10^{-4}$ for $\mathcal{O}_{2}$. In addition, there is a loop suppression for $\mathcal{O}_{1}$ where the decay has to proceed through an off-shell top quark. Without these additional suppressions, the bounds from indirect DM detection would require about two orders larger NP scale, $\Lambda \gtrsim 4.3 \cdot 10^{9} \mathrm{TeV}$.

The suppression factors are much larger for $B=2 \mathrm{DM}$, in which case the $\mathrm{DM}$ is a 
scalar, and the asymmetric operators start at dimension 10 . We investigate in detail the operator

$$
\begin{aligned}
\mathcal{O}_{1}^{(B=2)}=\chi\left(d_{K \alpha}^{c} d_{N \beta}^{c}\right) & \left(\left[q^{*} Y_{D}\right]_{M \alpha^{\prime}} q_{K^{\prime} \beta^{\prime}}^{*}\right)\left(q_{N^{\prime} \gamma^{\prime}}^{*} q_{M^{\prime} \gamma}^{*}\right) \epsilon^{K N M} \epsilon^{K^{\prime} N^{\prime} M^{\prime}} \epsilon^{\alpha \beta \gamma} \epsilon^{\alpha^{\prime} \beta^{\prime} \gamma^{\prime}} \\
\rightarrow & \chi\left(\left[d_{\mathrm{MASS}}^{c}\right]_{K \alpha}\left[d_{\mathrm{MASS}}^{c}\right]_{N \beta}\right)\left(\left[u_{\mathrm{MASS}}^{*} V_{\mathrm{CKM}}^{\dagger} Y_{D}^{\mathrm{diag}}\right]_{M \alpha^{\prime}}\left[d_{\mathrm{MASS}}^{*}\right]_{K^{\prime} \beta^{\prime}}\right) \\
& \times\left(\left[u_{\mathrm{MASS}}^{*} V_{\mathrm{CKM}}^{\dagger}\right]_{N^{\prime} \gamma^{\prime}}\left[d_{\mathrm{MASS}}^{*}\right]_{M^{\prime} \gamma}\right) \epsilon^{K N M} \epsilon^{K^{\prime} N^{\prime} M^{\prime}} \epsilon^{\alpha \beta \gamma} \epsilon^{\alpha^{\prime} \beta^{\prime} \gamma^{\prime}}
\end{aligned}
$$

that gives the least suppressed decay amplitude. Above, we chose one of the possible color contractions, implicitly assumed contractions of weak indices within brackets, and only kept the weak contraction leading to the largest decay rate in the second line.

The correct relic abundance requires a DM mass of $m_{\chi}=3.1 \pm 0.2 \mathrm{GeV}$, assuming the SM field content at the time of the decoupling of the asymmetric operators. We assume that $m_{\chi}<m_{\Lambda_{c}^{+}}+m_{\Sigma^{-}}=3.48 \mathrm{GeV}$, and thus below the threshold for the $\chi \rightarrow \Lambda_{c}^{+} \Sigma^{-}$decay, kinematically forbidding the $\chi \rightarrow u d c d d s$ partonic transition. The least suppressed partonic level transition is therefore $\chi \rightarrow u d s$ uds resulting, after hadronization, in the decays $\chi \rightarrow \Lambda^{0} \Lambda^{0}, \Sigma^{-} \Sigma^{+}, \Xi^{-} p, \Xi^{0} n, \ldots$ The NDA estimate of the $\chi$ decay width is then

$$
\Gamma_{\chi}^{(1)} \sim \frac{\left|y_{b} V_{u b}^{2}\right|^{2}}{8 \pi}\left(\frac{m_{\chi}}{\Lambda}\right)^{12} \frac{m_{\chi}}{\left(16 \pi^{2}\right)^{4}}=6.6 \cdot 10^{-51} \mathrm{GeV}\left(\frac{y_{b}}{0.024}\right)^{2}\left(\frac{0.63 \mathrm{TeV}}{\Lambda}\right)^{12}
$$

The MFV assumption results in the $y_{b} V_{u b}^{2}$ suppression of the Wilson coefficient. The $1 /\left(16 \pi^{2}\right)^{4}$ factor reflects the fact that, in the OPE, the leading contribution starts at 5 loops. The use of the OPE may be suspect for such low $m_{\chi}$ masses and one could expect $\mathcal{O}(1)$ corrections to the above estimate from additional soft gluon loops.

Indirect DM searches require the NP scale to be $\Lambda \gtrsim 0.49 \mathrm{TeV}$. This corresponds to the bounds on the masses of the mediators between the dark and the visible sectors, 


\begin{tabular}{ccccccccc}
\hline \hline \multicolumn{3}{c}{ ADM model } & \multicolumn{3}{c}{ MFV } & \multicolumn{3}{c}{ FN } \\
$B$ & Dim. & $m_{\chi}[\mathrm{GeV}]$ & decay & $\tau[\mathrm{s}]$ & $\Lambda[\mathrm{TeV}]$ & decay & $\tau[\mathrm{s}]$ & $\Lambda[\mathrm{TeV}]$ \\
\hline 1 & 6 & 6.2 & $\chi \rightarrow$ bus & $10^{26}$ & $4.0 \times 10^{6}$ & $\chi \rightarrow$ bus & $10^{26}$ & $8.1 \times 10^{8}$ \\
2 & 10 & 3.1 & $\chi \rightarrow$ udsuds & $10^{26}$ & 0.63 & $\chi \rightarrow$ udsuds & $10^{26}$ & 2.5 \\
3 & 15 & 2.1 & forbidden & $\infty$ & - & forbidden & $\infty$ & - \\
\hline \hline
\end{tabular}

Table 2.1: Leading decay modes for the $B=1,2,3$ ADM assuming MFV or FN flavor breaking. The dimensionality of the decaying operators are denoted in the 2nd column. With the suppression scales $\Lambda$ given in the 6th and 9th column the ADM decay time is $\tau \simeq 10^{26} \mathrm{~s}$. The $B=3 \mathrm{ADM}$ decays to quarks are kinematically forbidden.

$m_{\text {mediator }} \gtrsim 490 \mathrm{GeV}, m_{\text {mediator }} \gtrsim 210 \mathrm{GeV}$, and $m_{\text {mediator }} \gtrsim 90 \mathrm{GeV}$, if the operator (2.14) arises at tree level, 1-loop, or 2-loops, respectively. The mediators can thus be searched for at the LHC as discussed in Sec. 2.6.3. Note that the flavor suppression was essential to have such a low bound on the NP scale $\Lambda$. Without it, and taking the Wilson coefficient to be 1 , the indirect bounds on the stability of DM would require $\Lambda \gtrsim 7.3 \mathrm{TeV}$, implying that the mediators were most likely out of reach of the LHC.

The bound on the NP scale $\Lambda$ is quite sensitive to the actual value of $m_{\chi}$. For larger values of $m_{\chi}$, the $\chi$ can decay to top and bottom quarks reducing the loop and CKM suppression of the decay width. This is illustrated in Fig. 2.3, where the NP scale is fixed to $\Lambda_{\mathrm{MFV}}=1 \mathrm{TeV}$ and $m_{\chi}$ is varied. As the kinematic thresholds for the $\chi$ decays to $c$ or $b$ quarks are reached, this results in a change of several orders of magnitude in the predicted decay time.

\subsubsection{Spontaneously broken horizontal symmetries}

The suppression we found above using the MFV ansatz is model dependent. To illustrate this point we turn to $U(1)$ Frogatt-Nielsen (FN) models of spontaneously broken horizontal symmetries [68]. The suppression of the Wilson coefficients in the effective Lagrangian (2.7) is then given by the horizontal charges of the quarks in the operators. 
For instance, for the two $B=1$ DM operators in (2.7)

$$
\begin{aligned}
& \mathcal{O}_{1}^{(B=1)}=\left(\chi d_{K}^{c}\right)\left(u_{N}^{c} d_{M}^{c}\right) \rightarrow\left(\chi\left[d_{\mathrm{MASS}}^{c}\right]_{K}\right)\left(\left[u_{\mathrm{MASS}}^{c}\right]_{N}\left[d_{\mathrm{MASS}}^{c}\right]_{M}\right), \\
& \mathcal{O}_{2}^{(B=1)}=\left(\chi q_{K i}^{*}\right)\left(d_{N}^{c} q_{M j}^{*}\right) \epsilon^{i j} \rightarrow\left(\chi\left[u_{\mathrm{MASS}}^{*}\right]_{K}\right)\left(\left[d_{\mathrm{MASS}}^{c}\right]_{N}\left[d_{\mathrm{MASS}}^{*}\right]_{M}\right),
\end{aligned}
$$

the Wilson coefficients are

$$
C_{1} \sim \lambda^{\left|H\left(d_{K}^{c}\right)+H\left(u_{N}^{c}\right)+H\left(d_{M}^{c}\right)\right|}, \quad C_{2} \sim \lambda^{\left|-H\left(q_{K}\right)+H\left(d_{N}^{c}\right)-H\left(q_{M}\right)\right|} .
$$

Here $H\left(u_{K}^{c}\right), \ldots$, with $H\left(q_{K}^{*}\right)=-H\left(q_{K}\right)$, are the horizontal $U(1)$ charges of the quarks, and $\lambda \sim 0.2$ is the expansion parameter. The dependence of the operators and Wilson coefficients on the generational indices $K N M$ is implicit as are color, weak, and Lorentz contractions in (2.16).

An example of a horizontal charge assignment that gives phenomenologically satisfactory quark masses and CKM matrix elements is [69],

$$
H\left(q, d^{c}, u^{c}\right) \Rightarrow d^{c}\left(\begin{array}{ccc}
1 & 2 & 3 \\
3 & 2 & 0 \\
3 & 2 & 2 \\
3 & 1 & 0
\end{array}\right),
$$

where the column labels $\{1,2,3\}$ correspond to the first, second, and third generations of quarks.

Since the heavier flavors carry smaller charges the DM preferentially decays into the heaviest accessible states. As in MFV, the dominant decay is $\chi \rightarrow b u s$, except that the $y_{b} V_{u b} \sim \lambda^{5}$ suppression gets replaced by a much more modest $\sim \lambda^{\left|-H\left(q_{1}\right)+H\left(s^{c}\right)-H\left(q_{3}\right)\right|}=$ $\lambda$. This is the largest scaling allowed by FN charges. In concrete UV mediator models the suppression can, in fact, be much more severe as we will see explicitly in the next 
Section.

For $B=2 \mathrm{DM}$ the least suppressed operator is

$$
\begin{aligned}
\mathcal{O}_{1}^{(B=2)}=\chi\left(d_{K}^{c} d_{N}^{c}\right) & \left(q_{M}^{*} q_{K^{\prime}}^{*}\right)\left(q_{N^{\prime}}^{*} q_{M^{\prime}}^{*}\right) \\
& \rightarrow \chi\left(\left[d_{\text {MASS }}^{c}\right]_{K}\left[d_{\text {MASS }}^{c}\right]_{N}\right)\left(\left[u_{\text {MASS }}^{*}\right]_{M}\left[d_{\text {MASS }}^{*}\right]_{K^{\prime}}\right)\left(\left[u_{\text {MASS }}^{*}\right]_{N^{\prime}}\left[d_{\text {MASS }}^{*}\right]_{M^{\prime}}\right)
\end{aligned}
$$

suppressing, again, the color and weak contractions. The corresponding Wilson coefficient is suppressed by

$$
C_{1} \sim \lambda^{\left|H\left(d_{K}^{c}\right)+H\left(d_{N}^{c}\right)-H\left(q_{M}\right)-H\left(q_{K^{\prime}}\right)-H\left(q_{N^{\prime}}\right)-H\left(q_{M^{\prime}}\right)\right|} .
$$

At the partonic level, the dominant decay is $\chi \rightarrow$ uss uds with a Wilson coefficient that is of parametric size $\sim \lambda^{\left|H\left(d^{c}\right)+H\left(s^{c}\right)-2 H\left(q_{2}\right)-2 H\left(q_{1}\right)\right|}=\lambda^{5}$. Note that in MFV this process proceeded through 2 loops so that the suppression was much more severe, $\sim V_{t s} V_{u b} /\left(16 \pi^{2}\right)^{2} \sim \lambda^{5} /\left(16 \pi^{2}\right)^{2}$ at the amplitude level. While the suppression in the FN case is much less than in the MFV case, it is still nontrivial. It lowers the scale of NP allowed by indirect DM searches from $\Lambda \gtrsim 7.3 \mathrm{TeV}$, in the case of no flavor structure, to $\Lambda \gtrsim 2.5 \mathrm{TeV}$ in the FN case. Taking the bound from DM indirect detection searches gives $\Lambda \gtrsim 1.9 \mathrm{TeV}$. If the operator arises at tree level, 1-loop or 2-loops, this corresponds

to mediator masses, $m_{\text {mediator }} \gtrsim 1.9 \mathrm{TeV}, m_{\text {mediator }} \gtrsim 830 \mathrm{GeV}$, and $m_{\text {mediator }} \gtrsim 360 \mathrm{GeV}$, respectively.

\subsection{Indirect detection}

The asymmetric operators discussed in the previous section lead to a decaying DM which can be potentially seen in indirect DM searches. In our models, the $\chi$ decays hadronicaly. The decay products thus contain a number of charged particles and photons. The flavor composition of the final state depends on the mass, $m_{\chi}$, and also on 


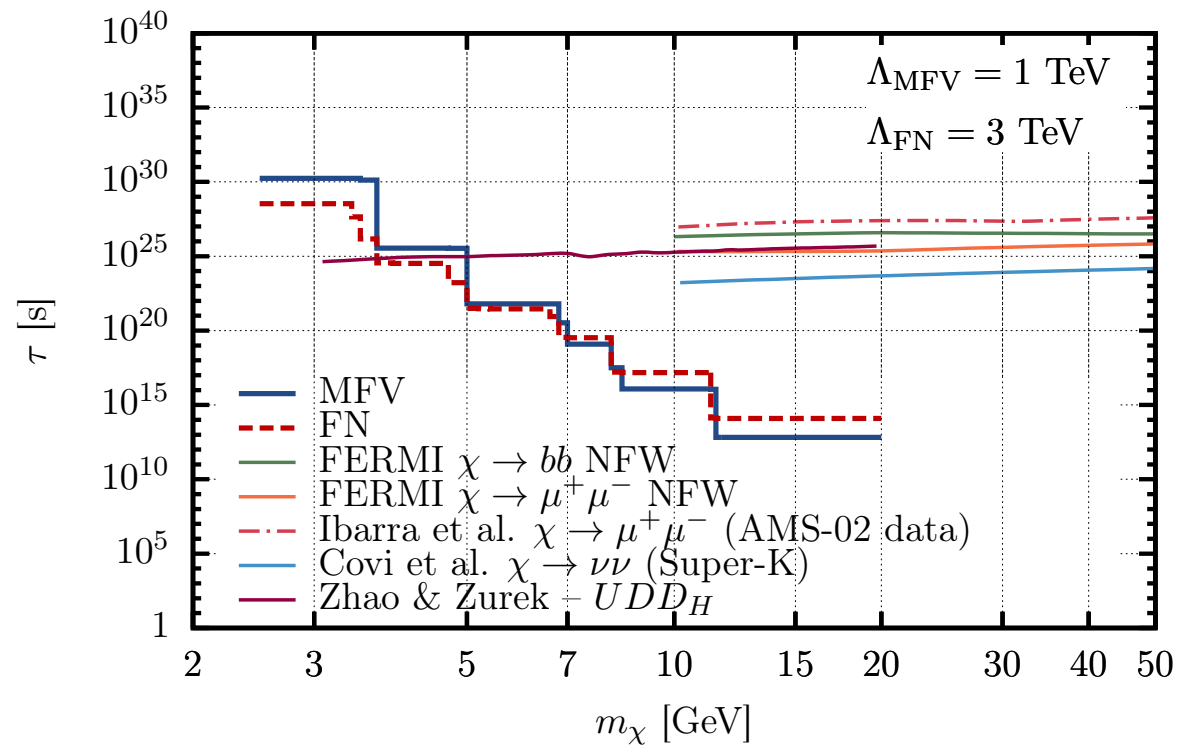

Figure 2.3: The solid blue (red dashed) line denotes the $B=2 \mathrm{DM}$ lifetime as a function of $m_{\chi}$ for the MFV (FN) case, fixing the NP scale to $\Lambda=1(3) \mathrm{TeV}$. Assuming the dominance of one decay mode, the green (orange) line shows the constraint on the decay time from FERMI-LAT [70] for $b \bar{b}\left(\mu^{+} \mu^{-}\right)$final states using the NFW profile. The dashdotted red line shows the AMS-02 [71] constraint on $\chi \rightarrow \mu^{+} \mu^{-}$decay time derived in [72], while the light blue line shows the Super-Kamiokande [73] constraint on the $\chi \rightarrow \nu \bar{\nu}$ decay time obtained in [74]. The purple line shows the upper limit on $\chi \rightarrow u d s$ and $\chi \rightarrow c b s$ decay times (indistinguishable at the scale of the figure) obtained in [37].

the assumed flavor breaking pattern. In Section 2.3, we discussed in detail the case of 6.2 GeV $B=1 \mathrm{DM}$, which decays through $\chi \rightarrow$ bus and a $3.1 \mathrm{GeV} B=2 \mathrm{DM}$ that decays through $\chi \rightarrow u d s u d s$. After hadronization, these result in the decays $\chi \rightarrow \Xi_{b}^{0} \pi^{0}$ and $\chi \rightarrow \Lambda^{0} \Lambda^{0}$, respectively. The dominant decays for other DM masses, assuming the MFV or FN flavor breaking patterns, are given in Appendix A.III. The DM lifetime dependence on $m_{\chi}$ is shown in Fig. 2.3 after fixing the NP scale to be $\Lambda=1(3) \mathrm{TeV}$ for the MFV (FN) flavor breaking.

To guide the eye, we also show in Fig. 2.3 the following bounds from indirect DM searches. The green (orange) line shows the constraint on the DM decay time from FERMI-LAT [70] for $\chi \rightarrow b \bar{b}\left(\mu^{+} \mu^{-}\right)$decays using the NFW profile. The dash-dotted 
light red line shows the results of an analysis [72] based on AMS-02 [71] and assuming $\chi \rightarrow \mu^{+} \mu^{-}$. The light blue line shows the result of an analysis [74] assuming $\chi \rightarrow$ $\bar{\nu} \nu$ decay based on Super-Kamiokande [73] bounds. The purple line is an exclusion curve from [37] based on galactic and extragalactic gamma ray flux measurements by Fermi [75-77]. The authors in [37] consider $\chi \rightarrow u d s$ and $\chi \rightarrow c b s$ decays as two extreme choices for the flavor structure of the final states. The derived bounds on the $\chi$ lifetime differ by less then a factor of 2 such that the two bounds overlap on the scale of Fig. 2.3. The decays we consider fall between these two extreme choices with potentially weakened bounds in our cases above $m_{\chi} \gtrsim \mathcal{O}(10) \mathrm{GeV}$ due to the increased multiplicity of final states. The bounds cross the expected $\chi$ decay times at $m_{\chi} \sim 5 \mathrm{GeV}$ for $\Lambda_{\mathrm{MFV}}=1 \mathrm{TeV}$ suppression scale in the case of MFV flavor breaking and at $m_{\chi} \sim 4$ $\mathrm{GeV}$ for $\Lambda_{\mathrm{FN}}=3 \mathrm{TeV}$ suppression scale in the case of FN flavor breaking.

For the $3.1 \mathrm{GeV} B=2 \mathrm{DM}$, we thus find that, for the MFV case, the indirect detection requires

$$
\Lambda_{\mathrm{MFV}} \gtrsim 0.49 \mathrm{TeV}
$$

where the dominant operator is given in (2.14). For the FN case the bound is

$$
\Lambda_{\mathrm{FN}} \gtrsim 1.9 \mathrm{TeV}
$$

where the least suppressed operator is given in (2.19).

\subsection{Mediator models}

The EFT analysis of metastable ADM using asymmetric operators is an appropriate approach to derive the indirect DM detection signatures as we did in the previous section. However, for DM direct detection searches and the DM production at colliders, the dominant signals are due to either a single mediator exchange or from direct pro- 


\begin{tabular}{cccccc}
\hline \hline Field & $S U(3)_{C}$ & $S U(2)_{L}$ & $U(1)_{Y}$ & $G_{F}$ & $U(1)_{B-L}$ \\
\hline$\phi_{L}$ & $\overline{\mathbf{3}}$ & $\mathbf{1}$ & $1 / 3$ & $(\mathbf{6}, \mathbf{1}, \mathbf{1})$ & $2 / 3$ \\
$\varphi_{L}$ & $\mathbf{6}$ & $\mathbf{1}$ & $1 / 3$ & $(\overline{\mathbf{3}}, \mathbf{1}, \mathbf{1})$ & $2 / 3$ \\
$\phi_{R}$ & $\overline{\mathbf{3}}$ & $\mathbf{1}$ & $-2 / 3$ & $(\overline{\mathbf{3}}, \mathbf{1}, \mathbf{1})$ & $2 / 3$ \\
\hline \hline
\end{tabular}

Table 2.2: The gauge and global charge assignment for the three scalar mediators, $\phi_{L}$, $\varphi_{L}$ and $\phi_{R}$, in the first UV completion toy model for which we assume the MFV flavor breaking pattern.

duction of the mediators. To assess the reach of these DM searches, the UV completions to our models are therefore needed.

We introduce two toy model UV completions that can generate the dimension 10 effective operators; that is, the operator in Eq. (2.14) for the MFV case and the operator in Eq. (2.19) for the FN case. The EFT operators are generated when the $\sim \mathrm{TeV}$ mediators are integrated out. In our first model, all the mediators are scalars, while in the second model there is also a fermionic mediator. The flavor structure in either of the two models could be of the MFV or of the FN type. For concreteness we fix the first model to have the MFV flavor breaking, and the second model to have the FN flavor breaking.

\subsubsection{MFV model with scalar mediators}

The SM is extended by the DM, $\chi$, and three flavor multiplets of scalar mediators - a color anti-triplet $\phi_{L}$ and a color sextet $\varphi_{L}$, both with hypercharge $1 / 3$, and a color sextet $\phi_{R}$ with hypercharge $-2 / 3$ (see Table 2.2). They transform under the flavor group $G_{F}$ as $(\mathbf{6}, \mathbf{1}, \mathbf{1}),(\overline{\mathbf{3}}, \mathbf{1}, \mathbf{1})$, and $(\overline{\mathbf{3}}, \mathbf{1}, \mathbf{1})$, respectively. The interaction Lagrangian between 
mediators and the SM is thus given by

$$
\begin{aligned}
\mathcal{L}_{\mathrm{INT}} & \supset \frac{\kappa_{1}}{2} \bar{K}_{I}^{A B}\left[\phi_{L}\right]_{\gamma}^{I}\left(q_{A, \alpha i}^{*} q_{B, \beta j}^{*}\right) \epsilon^{i j} \epsilon^{\alpha \beta \gamma}+\frac{\kappa_{2}}{2} \bar{K}_{\lambda}^{\alpha \beta}\left[\varphi_{L}\right]_{A}^{\lambda}\left(q_{B, \alpha i}^{*} q_{C, \beta j}^{*}\right) \epsilon^{i j} \epsilon^{A B C} \\
& +\frac{\kappa_{3}}{2}\left[Y_{D}\right]_{X}^{A}\left[\phi_{R}\right]_{A, \alpha}\left(d_{Y, \beta}^{c} d_{Z, \gamma}^{c}\right) \epsilon^{\alpha \beta \gamma} \epsilon^{X Y Z}+\kappa_{4} \bar{K}_{I}^{A B} \bar{K}_{\lambda}^{\alpha \beta} \chi^{\dagger}\left[\phi_{L}\right]_{\alpha}^{I}\left[\varphi_{L}\right]_{A}^{\lambda}\left[\phi_{R}\right]_{B, \beta}+\text { h.c. },
\end{aligned}
$$

where the flavor indices $A, B, C$ belong to $S U(3)_{Q}$ and $X, Y, Z$ to $S U(3)_{D}$. The QCD indices are $\alpha \beta \gamma$, while the weak isospin indices are denoted by $i, j$. The flavor index $I$ and color index $\lambda$ run from 1 to 6 . The matrices of the Clebsch-Gordan coefficients, $\bar{K}_{I}^{A B}$ and $\bar{K}_{\lambda}^{\alpha \beta}$, are the same as in [78] and satisfy the completeness relation $\left(\bar{K}_{I}^{A B}\right)^{*} \bar{K}_{I}^{C D}=\frac{1}{2}\left(\delta_{A}^{D} \delta_{B}^{C}+\delta_{A}^{C} \delta_{B}^{D}\right)$, and similary for $\bar{K}_{\lambda}^{\alpha \beta}$. In the second line of (2.23), the down Yukawa insertions make the interaction term with right-handed down quarks formally invariant under $G_{F}$.

Integrating out the mediators $\phi_{L, R}, \varphi_{L}$, gives the $\chi$ decay operator (2.14), with the Wilson coefficient

$$
\frac{\mathcal{C}_{1}}{\Lambda^{6}}=-\frac{1}{8} \frac{\kappa_{1} \kappa_{2} \kappa_{3} \kappa_{4}}{m_{\phi_{L}}^{2} m_{\varphi_{L}}^{2} m_{\phi_{R}}^{2}} .
$$

For $\kappa_{1}=\kappa_{2}=\kappa_{3}=\kappa_{4}=1$ the bounds from indirect DM searches thus require $m_{\phi_{L}, \phi_{R}, \varphi_{L}} \gtrsim 450 \mathrm{GeV}$, if all the mediator masses are the same. This should be appropriately rescaled if either $\kappa_{i}$ have smaller values or if all masses are not the same. For instance, for $\kappa_{i}=0.3$ the mass degenerate case of the mediators is bounded from below by $m_{\phi_{L}, \phi_{R}, \varphi_{L}} \gtrsim 200 \mathrm{GeV}$. Since the mediators carry color charges, they can be searched for at the LHC as discussed in Section 2.6.3 below.

Note that, for the Lagrangian in Eq. (2.23) the common scenario where the symmetric component of $\chi$ density annihilates through a dark photon [19,59-61] is phenomenologically not viable. In this case, at least some of the SM quark fields would need to carry a dark $U(1)$ charge in conflict with the low energy constraints if dark photon is light. A viable possibility, on the other hand, is the annihilation of $\chi \chi^{\dagger}$ to a 

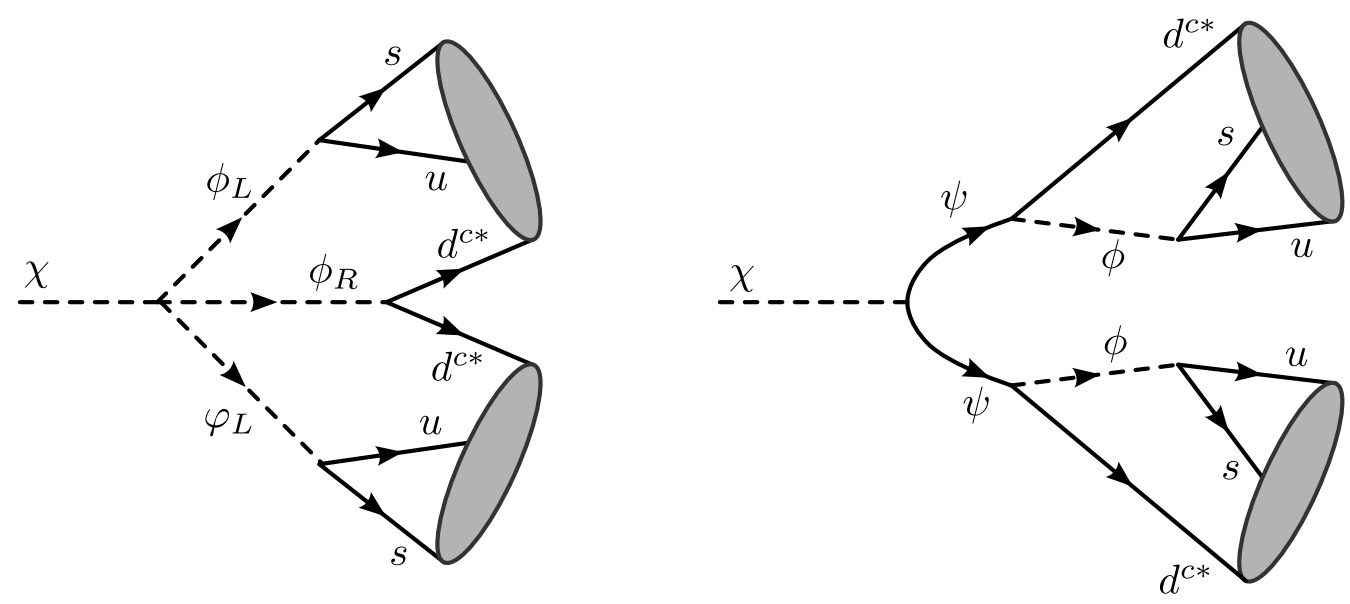

Figure 2.4: The $\chi$ decay in the MFV mediator model through the off-shell scalar mediators $\phi_{L, R}, \varphi_{L}$ (left), and through the off-shell fermion $\psi$ and scalar $\phi$ mediators in the FN model (right).

pair of light scalars along the lines of Ref. [79].

\subsubsection{FN model with fermionic and scalar mediators}

In the second model the SM is supplemented with a DM scalar $\chi$, a Dirac fermion $\psi$ and a complex scalar $\phi$ with SM gauge assignments as in Table 2.3. The relevant terms in the baryon number conserving interaction Lagrangian are

$$
\mathcal{L}_{\mathrm{INT}} \supset \frac{g_{q, A B}}{2} \phi_{\gamma}\left(q_{A, \alpha i}^{* j} q_{B, \beta j}^{* k}\right) \epsilon^{i j} \epsilon^{\alpha \beta \gamma}+g_{d, A} \phi^{* \alpha}\left(d_{A, \alpha}^{c} \psi\right)+\frac{g_{\chi}}{2} \chi\left(\psi^{c} \psi^{c}\right)+\text { h.c. },
$$

\begin{tabular}{ccccc}
\hline \hline Field & $S U(3)_{C}$ & $S U(2)_{L}$ & $U(1)_{Y}$ & $U(1)_{B-L}$ \\
\hline$\phi$ & $\overline{\mathbf{3}}$ & $\mathbf{1}$ & $1 / 3$ & $2 / 3$ \\
$\psi$ & $\mathbf{1}$ & $\mathbf{1}$ & 0 & 1 \\
\hline \hline
\end{tabular}

Table 2.3: Gauge and $B-L$ charges of the mediators $\phi$ and $\psi$ in the second UV completion toy model. We also assume the FN flavor breaking pattern. 
where, for the couplings $g_{q}, g_{d}$, we also denote the flavor dependence. If the flavor breaking is of the FN type and the mediators do not carry a horizontal charge, then

$$
g_{q, A B} \sim g_{q} \lambda^{\left|H\left(q_{A}\right)+H\left(q_{B}\right)\right|}, \quad g_{d, A} \sim g_{d} \lambda^{\left|H\left(d_{A}\right)\right|},
$$

where $g_{q, d} \sim \mathcal{O}(1)$.

Integrating out the mediators generates the operator (2.19) with the Wilson coefficient

$\frac{\mathcal{C}}{\Lambda^{6}} \simeq \frac{1}{8 m_{\psi}^{2} m_{\phi}^{4}} g_{\chi} g_{q, M, K^{\prime}} g_{q, N^{\prime} M^{\prime}} g_{d, K} g_{d, N} \sim \frac{1}{8 m_{\psi}^{2} m_{\phi}^{4}} \lambda^{\left|H\left(d_{K}^{c}\right)\right|+\left|H\left(d_{N}^{c}\right)\right|+\left|H\left(q_{M}\right)+H\left(q_{K^{\prime}}\right)\right|+\left|H\left(q_{N}^{\prime}\right)+H\left(q_{M^{\prime}}\right)\right|}$

Note that the flavor suppression here is parametrically different than in (2.20) which was obtained by assuming that the FN scale is close to the $\mathrm{TeV}$ scale and that the interactions of DM with the visible sector involve the FN fields. In the above model, however, the FN scale can be arbitrarily high and only fixes the flavor interactions between the mediator and the SM fields. Consequently, the leading decay is now $\chi \rightarrow$ ussuds where the suppression for the amplitude is $\sim \lambda^{\left|H\left(d^{c}\right)\right|+\left|H\left(s^{c}\right)\right|+2\left|H\left(q_{2}\right)+H\left(q_{1}\right)\right|} \sim \lambda^{15}$, to be compared with the $\lambda^{4}$ suppression in the more conservative case considered in Section 2.3.2 where the leading decay is $\chi \rightarrow u d s u d s$. The indirect detection bound (2.22) thus translates in our toy mediator model to $m_{\phi, \psi} \gtrsim 130 \mathrm{GeV}$ for mass degenerate $\phi$ and $\psi$. However, since the coupling to the third generation quarks is $\mathcal{O}(1)$, the scalar mediators should in fact be heavier than the top quark in order not to modify its total decay width.

The scaling (2.26) changes if the mediators carry nonzero horizontal charges. For instance, if the horizontal charge of $\phi$ is nonzero, $H(\phi) \neq 0$, one has $g_{q, A B} \sim \lambda^{\left|H\left(q_{A}\right)+H\left(q_{B}\right)-H(\phi)\right|}$, $g_{d, A} \sim \lambda^{\left|H\left(d_{A}\right)+H(\phi)\right|}$. In this case, the indirect detection bounds need to be appropriately rescaled. For $-2 \leq H(\phi) \leq 5$ the Wilson coefficient is still given by (2.27) and thus 
$m_{\phi, \psi} \gtrsim 130 \mathrm{GeV}$ from indirect bounds as before. For other values of $H(\phi)$, the bound becomes even weaker.

As far as the annihilation of symmetric part of the $\chi$ relic density is concerned, similar comments as for the MFV model in Section 2.5.1 apply. The dark U(1) is phenomenologically not viable, while annihilation to light scalars is. Furthermore, if $\psi$ has a mass within $\mathcal{O}(10 \%)$ of $m_{\chi}$, the process $\chi \chi^{\dagger} \rightarrow \psi \psi^{\dagger}$, which is forbidden at zero temperature but allowed for nonzero temperatures at the freeze-out, can efficiently annihilate away the symmetric component of $\chi$ without any need for additional states.

\subsection{Experimental signatures of the mediators}

Now we turn to the experimental signatures of weak scale mediators, the flavor constraints, direct DM detection, and DM production at the LHC.

\subsubsection{Flavor constraints}

The two mediator models from Sec. 2.5 do not lead to tree level flavor changing neutral currents (FCNCs). These are first generated at 1-loop, see Fig. 2.5. For real couplings $\kappa_{i}$ and $g_{q / d}$ in Eqs. (2.23) and (2.25), the constraints from $K^{0}-\bar{K}^{0}, D^{0}-\bar{D}^{0}$ and $B_{(s)}^{0}-\bar{B}_{(s)}^{0}$ mixing require the mediators masses to be generically above several hundred $\mathrm{GeV}$ as we show below. For related analyses of flavor constraints on diquarks, see, e.g., [80,81].

The $\Delta F=2$ effective weak Hamiltonian is

$$
\mathcal{H}_{\mathrm{eff}}^{\Delta F=2}=\sum_{i} \mathcal{C}_{i} \mathcal{O}_{i}+\sum_{i} \tilde{\mathcal{C}}_{i} \tilde{\mathcal{O}}_{i}
$$

where $i=1, \ldots, 5$ runs over the dimension six operators (we use the notation in [82]). Integrating out the mediators and the $W$ at the weak scale gives, at leading order, a 

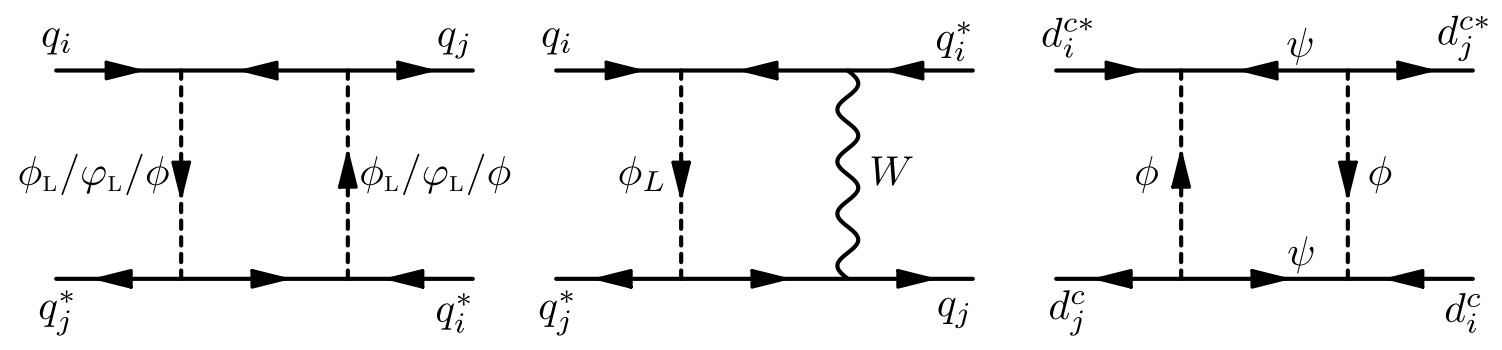

Figure 2.5: Box diagrams contributing to the neutral meson mixing. In the MFV model, there is also a contribution with both $\phi_{L}$ and $\varphi_{L}$ in the loop, while $\phi_{R}$ contributions are suppressed and can be ignored.

nonzero Wilson coefficient for the operator

$$
\mathcal{O}_{1}=\left(\bar{S}^{\alpha} \gamma_{\mu} P_{L} D^{\alpha}\right)\left(\bar{S}^{\beta} \gamma^{\mu} P_{L} D^{\beta}\right)=\left(s^{* \alpha} \bar{\sigma}^{\mu} d^{\alpha}\right)\left(s^{* \beta} \bar{\sigma}_{\mu} d^{\beta}\right),
$$

in the case of the MFV model, and for both $\mathcal{O}_{1}$ and its parity conjugate operator

$$
\tilde{\mathcal{O}}_{1}=\left(\bar{S}^{\alpha} \gamma_{\mu} P_{R} D^{\alpha}\right)\left(\bar{S}^{\beta} \gamma^{\mu} P_{R} D^{\beta}\right)=\left(s^{c \alpha} \bar{\sigma}^{\mu} d^{c \alpha *}\right)\left(s^{c \beta} \bar{\sigma}_{\mu} d^{c \beta *}\right),
$$

in the case of the FN model. Above, we first give the operators in the 4-component notation and then also in the 2-component notation (for our notation see Appendix A.I).

In the matching there are two types of contributions: in the first, only the mediators run in the loop whereas in the second, both the scalar mediator and the $W$ boson run in the loop, see Fig. 2.5. For the MFV model, these give for the $K^{0}-\bar{K}^{0}, D^{0}-\bar{D}^{0}$, and 


$$
\begin{aligned}
B_{(s)}-\bar{B}_{(s)} & \text { mixing } \\
\mathcal{C}_{1 K}^{\mathrm{MFV}}= & \frac{1}{64 \pi^{2} m_{\phi}^{2}}\left\{\left(V_{c s} V_{c d}^{*}\right)^{2}\left[\left(\kappa_{1}^{4}+3 \kappa_{2}^{4}-2 \kappa_{1}^{2} \kappa_{2}^{2}\right) F\left(x_{c}\right)+4 g_{w}^{2} \kappa_{2}^{2} G\left(x_{w}, x_{c}\right)\right]+c \rightarrow t\right. \\
& \left.-2 V_{c s} V_{c d}^{*} V_{t s} V_{t d}^{*}\left[\left(\kappa_{1}^{4}+3 \kappa_{2}^{4}-2 \kappa_{1}^{2} \kappa_{2}^{2}\right) F^{F}\left(x_{c}, x_{t}\right)+4 g_{w}^{2} \kappa_{2}^{2} G^{F}\left(x_{w}, x_{c}, x_{t}\right)\right]\right\} \\
\mathcal{C}_{1 D}^{\mathrm{MFV}}= & \frac{1}{64 \pi^{2} m_{\phi}^{2}}\left\{\left(V_{u s} V_{c s}^{*}\right)^{2}\left[\left(\kappa_{1}^{4}+3 \kappa_{2}^{4}-2 \kappa_{1}^{2} \kappa_{2}^{2}\right) F\left(x_{s}\right)+4 g_{w}^{2} \kappa_{2}^{2} G\left(x_{w}, x_{s}\right)\right]+s \rightarrow b\right. \\
& \left.-2 V_{u s} V_{c s}^{*} V_{u b} V_{c b}^{*}\left[\left(\kappa_{1}^{4}+3 \kappa_{2}^{4}-2 \kappa_{1}^{2} \kappa_{2}^{2}\right) F^{F}\left(x_{s}, x_{b}\right)+4 g_{w}^{2} \kappa_{2}^{2} G^{F}\left(x_{w}, x_{s}, x_{b}\right)\right]\right\} \\
\mathcal{C}_{1 B_{q}}^{\mathrm{MFV}=} & \frac{1}{64 \pi^{2} m_{\phi}^{2}}\left(V_{t b} V_{t q}^{*}\right)^{2}\left[\left(\kappa_{1}^{4}+3 \kappa_{2}^{4}-2 \kappa_{1}^{2} \kappa_{2}^{2}\right) F\left(x_{t}\right)+4 g_{w}^{2} \kappa_{2}^{2} G\left(x_{w}, x_{t}\right)\right],
\end{aligned}
$$

where $q=d, s, x_{i}=\left(m_{i} / m_{\phi}\right)^{2}$, and we have set $m_{u}=m_{d}=0$ and assumed, for simplicity, that the $\phi$ and $\varphi$ are mass degenerate. The loop functions $F(x), F^{F}\left(x_{1}, x_{2}\right), G\left(x_{1}, x_{2}\right)$, and $G^{F}\left(x_{1}, x_{2}, x_{3}\right)$ are given in Appendix A.IV. As in the SM, the largest contribution to the $K^{0}-\bar{K}^{0}$ mixing is due to the charm-charm loop while, for $B_{q}^{0}-\bar{B}_{q}{ }^{0}$ mixing, the top loop dominates as expected.

For the FN model, the Wilson coefficients are given by

$$
\begin{aligned}
\mathcal{C}_{1 K}^{\mathrm{FN}} \sim \frac{\lambda^{10} g_{q}^{4}}{16 \pi^{2} m_{\phi}^{2}}\left[H\left(x_{t}\right)+2 \lambda^{4} H^{F}\left(x_{c}, x_{t}\right)\right], & \tilde{\mathcal{C}}_{1 K}^{\mathrm{FN}} \sim \frac{\lambda^{10} g_{d}^{4}}{16 \pi^{2} m_{\phi}^{2}} H\left(x_{\psi}\right), \\
\mathcal{C}_{1 B_{d}}^{\mathrm{FN}} \sim \frac{\lambda^{6} g_{q}^{4}}{16 \pi^{2} m_{\phi}^{2}}\left[H\left(x_{t}\right)+2 \lambda^{4} H^{F}\left(x_{c}, x_{t}\right)\right], & \tilde{\mathcal{C}}_{1 B_{d}}^{\mathrm{FN}} \sim \frac{\lambda^{10} g_{d}^{4}}{16 \pi^{2} m_{\phi}^{2}} H\left(x_{\psi}\right), \\
\mathcal{C}_{1 B_{s}}^{\mathrm{FN}} \sim \frac{\lambda^{4} g_{q}^{4}}{16 \pi^{2} m_{\phi}^{2}}\left[H\left(x_{t}\right)+2 \lambda^{4} H^{F}\left(x_{c}, x_{t}\right)\right], & \tilde{\mathcal{C}}_{1 B_{d}}^{\mathrm{FN}} \sim \frac{\lambda^{8} g_{d}^{4}}{16 \pi^{2} m_{\phi}^{2}} H\left(x_{\psi}\right),
\end{aligned}
$$

while $\mathcal{C}_{1 D}^{\mathrm{FN}}=\mathcal{C}_{1 K}^{\mathrm{FN}}$ and $\tilde{\mathcal{C}}_{1 D}^{\mathrm{FN}}=0$. Above, we have indicated the scaling of different contributions to the Wilson coefficient in terms of $\lambda=0.2$, cf. Sec. 2.3.2. In the numerics we use the equality sign. The loop functions $H(x)$ and $H^{F}\left(x_{1}, x_{2}\right)$ are given in Appendix A.IV . 


\begin{tabular}{ccccc}
\hline \hline & \multicolumn{2}{c}{ MFV } & \multicolumn{2}{c}{ FN } \\
& $\kappa_{1,2}<$ & $m_{\phi_{L}, \varphi_{L}}>$ & $g_{q, d}<$ & $m_{\phi}>$ \\
\hline$K^{0}-\bar{K}^{0}$ & 0.33 & $2.9 \mathrm{TeV}$ & 0.63 & $570 \mathrm{GeV}$ \\
$B_{d}-\bar{B}_{d}$ & 1.3 & $710 \mathrm{GeV}$ & 0.54 & $1 \mathrm{TeV}$ \\
$B_{s}-\bar{B}_{s}$ & 1.3 & $780 \mathrm{GeV}$ & 0.59 & $840 \mathrm{GeV}$ \\
$D^{0}-\bar{D}^{0}$ & 30 & $34 \mathrm{GeV}$ & 4.3 & $56 \mathrm{GeV}$ \\
\hline \hline
\end{tabular}

Table 2.4: The $95 \%$ C.L. bounds on the MFV and FN mediator models from meson mixing. Taking $m_{\phi_{L}}=m_{\varphi_{L}}=m_{\phi}=1 \mathrm{TeV}$ and $\kappa_{1}=\kappa_{2}\left(g_{q}=g_{d}\right)$ gives the upper bounds on the couplings in the 2 nd(4th) column. Taking in turn $\kappa_{1,2}=g_{q, d}=1$ gives lower bounds on the mediator masses in the 3 rd and 5th columns. The mass of the fermion in the FN model is fixed to $m_{\psi}=20 \mathrm{GeV}$ (see Sec. 2.6.3). The bounds are not very sensitive to $m_{\psi}$.

Note that the above Wilson coefficients contain $\log \left(m_{i} / m_{\phi}\right)$ that can become large for $m_{\phi} \gg m_{i}$. We do not attempt to resum these logarithms which also means that we treat all the NP contributions as local. We expect that our numerical results can receive $\mathcal{O}(1)$ corrections due to neglected terms. This is within the precision required for our analysis. Though, we do include the usual RGE effects due to the NLO QCD running of the effective weak Hamiltonian from the weak scale to the low energy. For constraints from $K^{0}-\bar{K}^{0}$ and $B_{(s)}-\bar{B}_{(s)}$ mixing we use the recent results of a fit to the mixing parameters in [83]. The constraints from $D^{-} \bar{D}^{0}$ mixing are obtained by assuming that the NP contribution saturates $\Delta m_{D}$ so that in the equation $x_{D}=$ $2\left|\left\langle\bar{D}^{0}\left|\mathcal{H}_{\text {eff }}^{\Delta C=2}\right| D^{0}\right\rangle\right| / \Gamma_{D}$, valid in the limit of no CP violation, we only include the NP contribution [82]. The resulting bounds on couplings and masses are shown in Tab. 2.4. In the case of the MFV model, the most severe bound comes from $K^{0}-\bar{K}^{0}$ and is due to $\epsilon_{K}$. Since we assume that all the $\kappa_{i}$ in (2.23) are real, the NP contribution does carry a weak phase due to the $V_{t s} V_{t d}^{*} \mathrm{CKM}$ factors and does contribute to $\epsilon_{K}$. In contrast, in the FN model the NP contributions to the mixing do not carry a weak phase and thus do not have an effect on $\epsilon_{K}$. Therefore, the bounds from $K^{0}-\bar{K}^{0}$ mixing are much less 

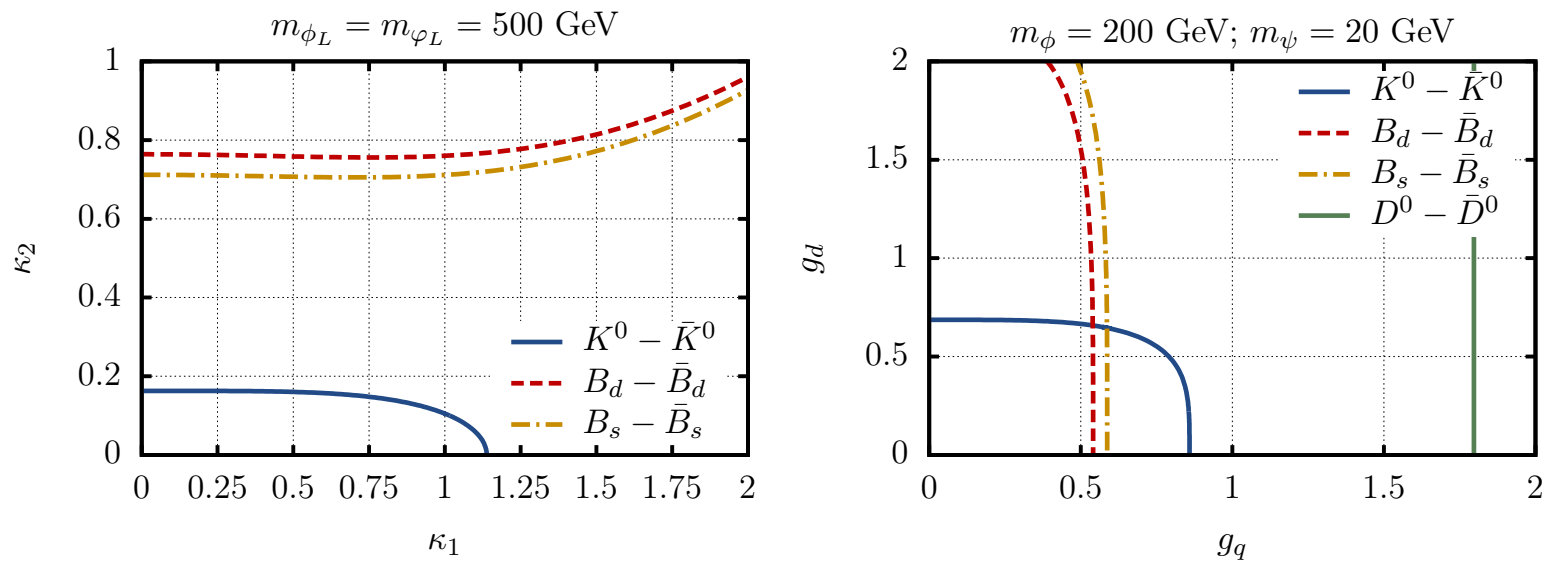

Figure 2.6: Meson mixing constraints on the couplings $\kappa_{1,2}$ in the MFV mediator model (left) and $g_{q, d}$ in the FN model (right), taking $m_{\phi_{L}}=m_{\varphi_{L}}=500 \mathrm{GeV}$ and $m_{\phi}=200$ $\mathrm{GeV}, m_{\psi}=20 \mathrm{GeV}$ respectively. The excluded regions lie above and to the right of the curves.

severe.

In Fig. 2.6, we show the constraint on the couplings $\kappa_{1,2}$ in the MFV model, fixing $m_{\phi_{L}}=m_{\varphi_{L}}=500 \mathrm{GeV}$ (left figure), and the constraints on $g_{q, d}$ in the FN model, fixing $m_{\phi}=200 \mathrm{GeV}, m_{\psi}=20 \mathrm{GeV}$ (right figure). Since, in the case of MFV, the largest contribution to $K^{0}-\bar{K}^{0}$ is from the mediator- $W$ loop, the $\epsilon_{K}$ bound places a stringent constraint on $\kappa_{2}$. Since the NP contributions to the meson mixing were assumed to be $\mathrm{CP}$ conserving in the case of the FN model, the couplings $g_{d, q} \sim \mathcal{O}(1)$ are allowed even for $m_{\phi}$ as low as $200 \mathrm{GeV}$.

\subsubsection{Relic abundance and direct detection}

We note in passing that the virtual exchanges of the mediators generate contact operators of the schematic form $\chi^{\dagger} \chi \bar{q} q$ that contribute to the $\chi \chi^{\dagger}$ annihilation cross section and to the cross section for DM scattering on nuclei. The symmetric couplings of DM and the mediators, of schematic form $\chi \chi^{\dagger} \phi \phi^{\dagger}$, do not suffice to create large enough annihilation cross sections that would annihilate away the symmetric component of DM relic abundance. 
As an example, consider the MFV model with scalar mediators, Eq. (2.23), and assume that the lightest mediator is $\phi_{L}$. It can have a symmetric coupling to DM of the form

$$
\mathcal{L} \supset \kappa^{\prime}\left[\phi_{L}\right]_{\gamma}^{I}\left[\phi_{L}^{\dagger}\right]_{\gamma}^{I} \chi^{\dagger} \chi
$$

At 1-loop, this generates a contact interaction $\chi^{\dagger} \partial_{\mu} \chi \bar{q} \gamma^{\mu} q$, which leads to an annihilation cross section $\langle\sigma v\rangle \sim \mathcal{O}\left(10^{-28} \mathrm{~cm}^{3} / \mathrm{s}\right)\left(100 \mathrm{GeV} / m_{\phi_{L}}\right)^{4}$ for $\mathcal{O}(1)$ couplings. This annihilation cross section is more than three orders of magnitude too small to obtain the observed relic density and satisfy CMB constraints for $s$-wave annihilation [59]. Thus, the symmetric component of the DM needs to annihilate away through a different mechanism as discussed at the end of Sec. 2.5.1.

\subsubsection{Collider signatures}

In both the MFV and FN flavor breaking scenarios, the mediator models involve colored scalars. These can be searched for at the LHC through the gluon initiated pair production or through a single production. We use our two mediator models to estimate the LHC reach. The MFV mediator model, Eq. (2.23), contains three colored scalars that are either triplets or sextets of the color and flavor groups, see Tab. 2.2. The FN model, Eq. (2.25), contains a colored scalar and a neutral fermion, see Table 2.3.

Pair production of colored scalars is the dominant production mechanism of the mediators for the masses of interest, below $\mathcal{O}(\mathrm{TeV})$. We illustrate this in Fig. 2.7 for the color triplet $\phi$ in the FN model where we compare the pair production cross section from gluon fusion and from quark-guon fusion, and the single production of $\phi$ in association with a jet. Gluon fusion clearly dominates in the mass range of interest.

The signatures of pair produced colored scalars depend on their decay modes. In our two models they decay either directly to two SM quarks or, alternatively, first to two lighter scalars that then in turn decay to two jets each. In the FN model the decay 


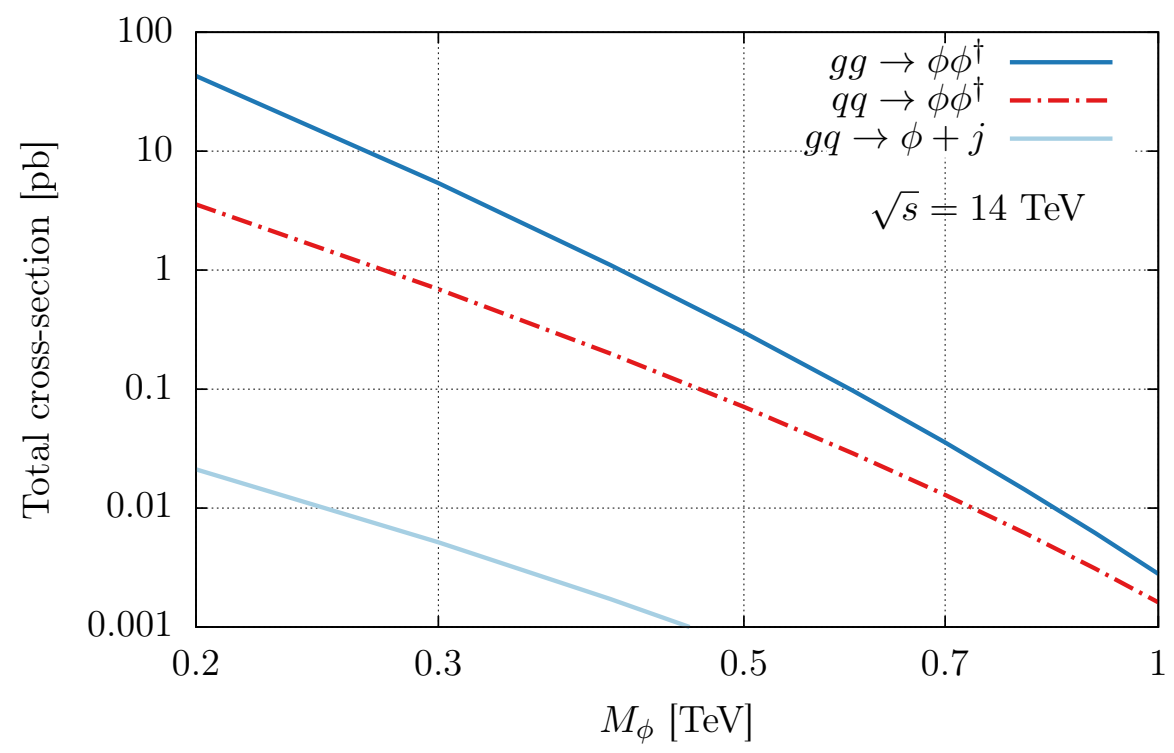

Figure 2.7: The $g g \rightarrow \phi \phi^{\dagger}$ (solid blue), $q q \rightarrow \phi \phi^{\dagger}$ (dot-dashed red) and $g q \rightarrow \phi j$ (solid light blue) contributions to the pair-production and single-production cross-section at the LHC with $\sqrt{s}=14 \mathrm{TeV}$ as a function of a mass of a color triplet scalar $\phi$, a mediator in the FN model.

$\phi \rightarrow j \psi$ is also possible. The flavor composition of the jets depends on the flavor quantum numbers of the scalar. For instance, the states in the $\phi_{L}$ flavor multiplet can decay either predominantly through $\phi_{L} \rightarrow t b, \phi_{L} \rightarrow b j$, or $\phi_{L} \rightarrow j j$, depending on the flavor numbers of $\phi_{L}$ (and similarly for $\varphi_{L}$ ), see Eq. (2.23). The scalars in the $\phi_{R}$ flavor multiplet, on the other hand, decay through $\phi_{R} \rightarrow b j$ or $\phi_{R} \rightarrow j j$, again depending on the flavor index carried by the $\phi_{R}$ state. In the FN model one needs to require $m_{\phi}>m_{t}$ in order not to modify the total decay width of the top quark, see Sec. 2.5. Then, the dominant decay is either $\phi \rightarrow \bar{b} \psi$ or $\phi \rightarrow t b$, depending on the relative sizes of the two couplings, while the other decays are suppressed by additional powers of $\lambda$.

To get a rough estimate of the LHC sensitivity we treat all the decay modes as two-jet final states (this overestimates the reach slightly since, for the $t j$ final state, the real efficiency is expected to be lower). The strongest constraint on pair-production of the lightest scalar mediators then comes from the search for pair-produced dijet 


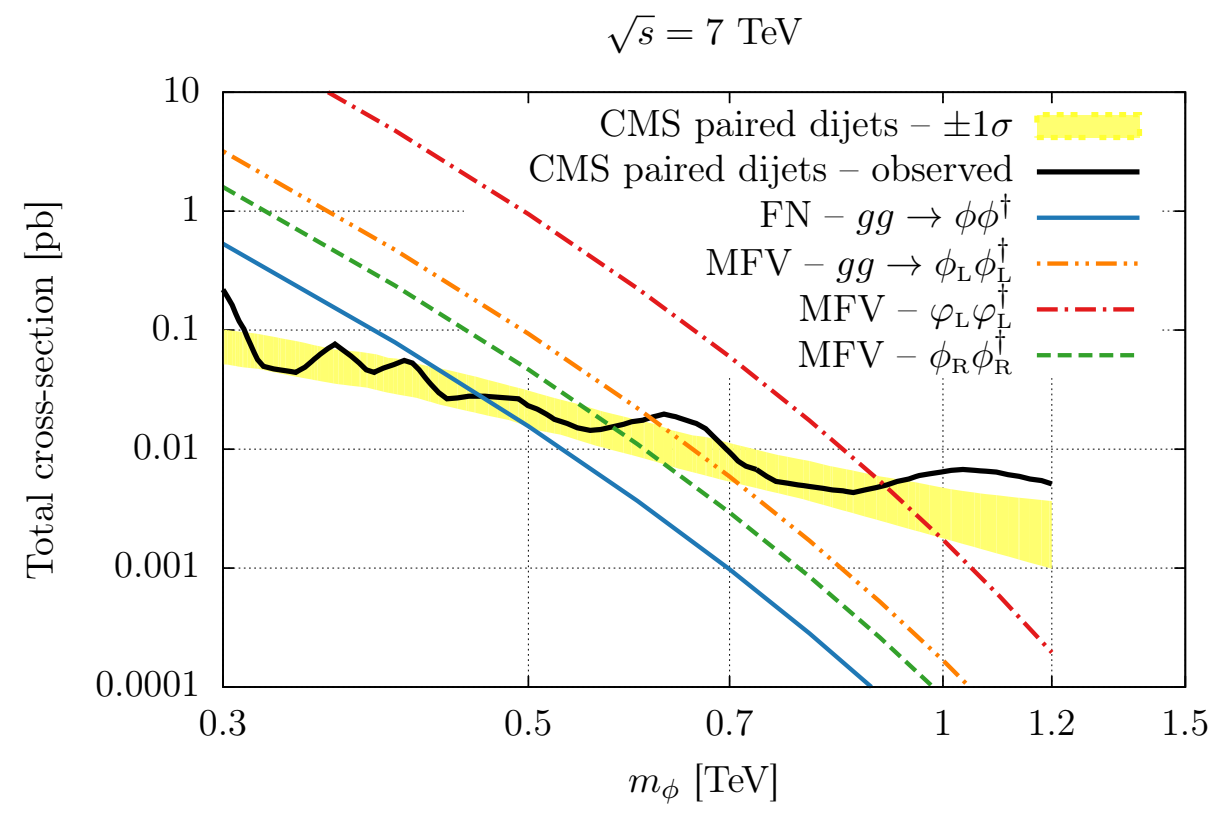

Figure 2.8: Constraints on the scalar mediator $\phi$ in the FN model, and $\phi_{L}, \varphi_{L}, \phi_{R}$ in the MFV model that follow from the CMS search for pair-produced dijet-resonances [84]. The states in the same flavor multiplet are taken to be mass-degenerate.

resonances from CMS at $7 \mathrm{TeV} \mathrm{LHC}$ with integrated luminosity of $5 \mathrm{fb}^{-1}$ [84]. This places the bounds $m_{\phi} \gtrsim 470 \mathrm{GeV}$ in the case of FN model assuming that $\phi \rightarrow \bar{b} \psi$ decay is negligible, and $m_{\phi_{L}} \gtrsim 620 \mathrm{GeV}, m_{\varphi_{L}} \gtrsim 910 \mathrm{GeV}, m_{\phi_{R}} \gtrsim 580 \mathrm{GeV}$ in the case of MFV flavor breaking as shown in Fig. 2.8. Note that when all three mediators are degenerate in mass, the color sextet scalar has the largest pair production cross section due to the large color factor.

In the FN model, a new experimental signature is obtained in the limit $g_{d} \gg \lambda^{2} g_{q}$. Then the dominant decay of $\phi$ is $\phi \rightarrow \bar{b} \psi$. In order not to have fast decaying DM $m_{\psi}>m_{\chi} / 2$. Using NDA the $\psi$ decay length is

$$
c \tau(\psi \rightarrow b b c) \sim\left(g_{q}^{2} g_{d}^{2} \lambda^{8} \frac{1}{8 \pi} \frac{1}{16 \pi^{2}} \frac{m_{\psi}^{5}}{m_{\phi}^{4}}\right)^{-1} \sim 30 \mathrm{~m}\left(\frac{20 \mathrm{GeV}}{m_{\psi}}\right)^{5}\left(\frac{m_{\phi}}{750 \mathrm{GeV}}\right)^{4}\left(\frac{0.03}{g_{q} g_{d}}\right)^{2} .
$$

For light enough $\psi$ (or heavy enough $\phi$ ), the fermion $\psi$ does not decay in the detec- 


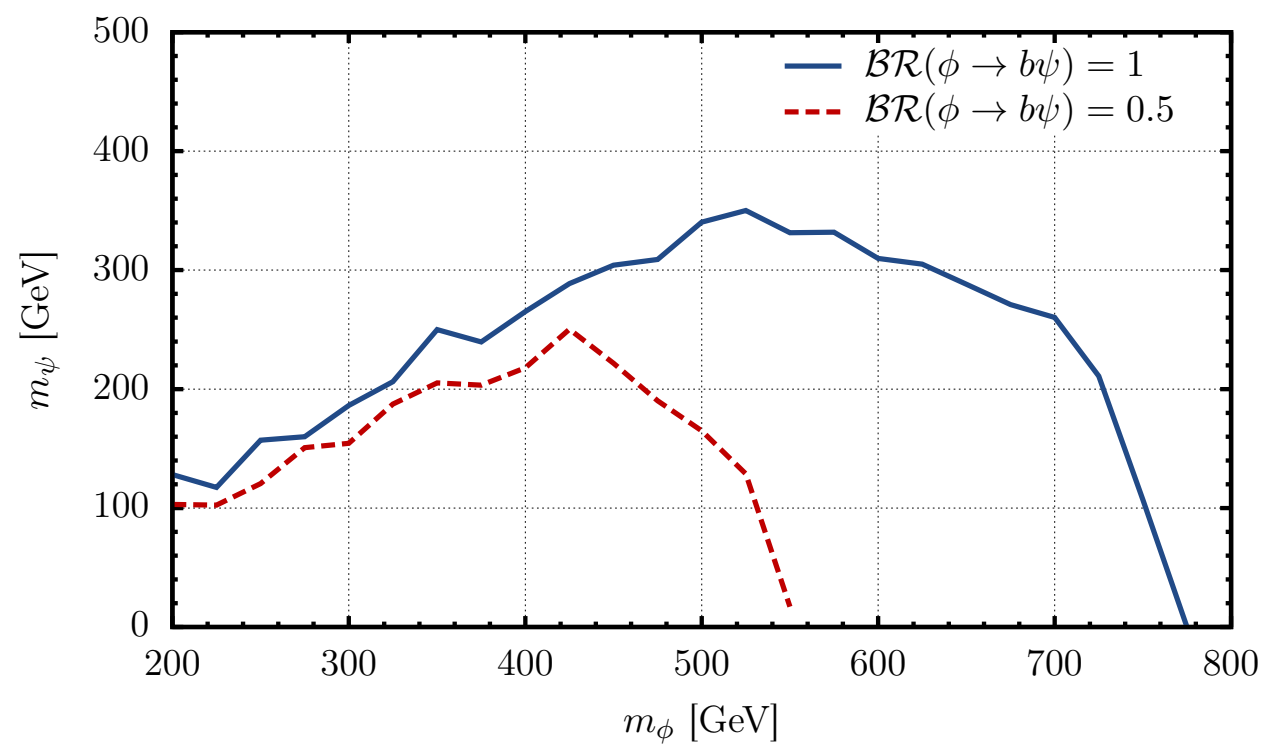

Figure 2.9: The 95\% exclusion limit on $\phi \phi^{\dagger}$ production in the FN model for the $b \bar{b} \psi \bar{\psi}$ final state, where $\psi$ escapes the detector and sbottom search applies [85]. The solid blue (dashed red) line is for $\phi \rightarrow b \psi$ branching ratios of $50 \%$ and $100 \%$.

tor and appears as $\mathscr{E}_{T}$. The $p p \rightarrow \phi \phi^{\dagger}$ pair production then results in $2 j+\mathscr{F}_{T}$ or $2 b+\mathscr{F}_{T}$ final state, and is bounded from sbottom searches as shown in Fig. 2.9. The resulting bound is $m_{\phi}>550(760) \mathrm{GeV}$ for $m_{\psi}=20 \mathrm{GeV}$ and $\mathcal{B R}(\phi \rightarrow \psi b)=0.5(1.0)$. The choice $g_{q}=g_{d}=0.03$ in Eq. (2.38) gives $\mathcal{B R}(\phi \rightarrow b \psi) \approx \mathcal{B R}(\phi \rightarrow s \psi)=0.33$. For the same input parameters, the single production of $\psi$ in association with $b, t$, or $\phi$ has a cross section $\sim 7 \cdot 10^{-2} \mathrm{fb}$ while the pair production is dominated by the process $s s \rightarrow \psi \psi$ and has a negligible cross section of $\sim 4 \cdot 10^{-4} \mathrm{fb}$.

The single production of mediators, e.g., $u d \rightarrow \phi, u d \rightarrow \phi_{L}, u d \rightarrow \varphi_{L}, d s \rightarrow \phi_{R}$, is suppressed due to the small couplings of the mediators to the first and the second generation quarks. Similarly, the single production from heavy quarks in the initial state suffers from the PDF suppression.

For single top production, the MFV model gives the largest contribution with a cross-section of $\sigma\left(u d \rightarrow \phi_{L} \rightarrow t b\right)_{\mathrm{MFv}}=7.6 \times 10^{-6} \mathrm{pb}$ for $m_{\phi_{L}}=500 \mathrm{GeV}$ and $\sqrt{s}=8$ 
$\mathrm{TeV}$. This is well below the SM production cross section. Thus, the ATLAS and CMS combined measurement of the single top cross section at $\sqrt{s}=8 \mathrm{TeV}, 85 \pm 12 \mathrm{pb}$ [86] and so does not impose any limits on the mediator model.

The production of the DM, $\chi$, can occur from the decay of heavier mediators. For instance, for $\kappa_{4} \sim \kappa_{3}$ and $\phi_{R}$ heavy enough, the dominant decay mode of $\phi_{3}$ is $\phi_{3} \rightarrow$ $\chi \varphi_{L}^{\dagger} \phi_{L}^{\dagger}$. Pair production $p p \rightarrow \phi_{R} \phi_{R}^{\dagger}$ would thus result in $8 j+\mathbb{F}_{T}$ signature where paired dijets would reconstruct $\phi_{L}$ and $\varphi_{L}$ mass peaks (depending on the flavor assignments some of the jets can be replaced by $t$ of $b$ jets).

\subsection{Chapter summary}

In this chapter, we have shown that for asymmetric DM (ADM) models, the stability of $\mathrm{DM}$ on cosmological time scales may be purely accidental. We did not require that the DM to be charged under an ad-hoc conserved $Z_{n}$ symmetry. Rather, we assumed that such a discrete symmetry is explicitly broken by the mediator interactions that transfer the $B-L$ between the DM sector and the visible sector in the early universe. Such asymmetric interactions are necessary in all models of ADM though they may be made to obey a $Z_{4}$ symmetry (i.e. one can demand that they involve only the $\chi \chi \rightarrow$ visible or $\chi^{\dagger} \chi^{\dagger} \rightarrow$ visible transitions instead of $\chi \rightarrow$ visible transitions as in our case).

At low energies, the DM then carries a conserved $\chi$ charge that is broken only by the higher dimensional operators obtained by integrating out the mediators. Such operators also lead to DM decays. In this chapter, we explored the role of continuous flavor symmetries for the properties of such decaying DM focusing on the case where $\mathrm{DM}$ that carries nonzero baryon number. For $B=1 \mathrm{DM}$, the direct detection bounds are evaded if the mediators are above $\sim 4 \cdot 10^{9} \mathrm{TeV}$ assuming $\mathcal{O}(1)$ couplings. However, if quark flavor breaking is of the MFV type, the mediators can be lighter by around two orders of magnitude. For $B=2 \mathrm{DM}$, the scale of the mediators can be much lighter 
$(\mathcal{O}(8 \mathrm{TeV})$ for $\mathcal{O}(1)$ couplings). This is then lowered by an order of magnitude if quark flavor breaking is of the MFV or Froggatt-Nielsen type. The mediators that would lead to indirect DM signals in the next generation of experiments can thus be, at the same time, searched for at the LHC.

We have explored this possibility by constructing two mediator models, one with assumed MFV and one with a FN flavor breaking pattern. The MFV mediator model (Eq. (2.23)) contains three colored scalars that are either triplets or sextets of the color and the flavor groups, see Table 2.2. The FN model (Eq. (2.25)), on the other hand, contains one colored scalar and one neutral fermion, see Table 2.3. These mediators generate FCNCs at 1-loop. While this leads to nontrivial constraints on their masses and couplings, the mediators can still be as light as a few $\times 100 \mathrm{GeV}$ with $\mathcal{O}(1)$ couplings. Since the mediators are charged under QCD, they can be singly or pair-produced at the LHC with large cross sections. This means that the searches at the LHC can lead to interesting constraints or discoveries. The signatures depend on how the mediators decay. In the FN model, for instance, the decay to heavy quarks, $\phi \rightarrow t b$, is favored. Modifying the paired dijet searches to the $p p \rightarrow \phi \phi \rightarrow t b \bar{t} \bar{b}$ signal could thus enhance the reach of the LHC in the search for these mediators. In the MFV model, on the other hand, paired light dijets, paired $t b$, and paired $b j$ are possible. Other signatures are discussed in Sec. 2.6.3.

In conclusion, $\mathrm{ADM}$ can quite generically be metastable with a possibility of complementary signals in indirect detection and at the LHC. 


\section{Chapter 3}

\section{Dark Matter And Gauged Flavor Symmetries}

\subsection{Introduction}

The stability of dark matter (DM) is commonly assumed to be due to an exact discrete symmetry, $\mathcal{Z}_{n}$. This can either be imposed by hand or have a dynamical origin. Examples include $R$-parity in the MSSM [87], and flavor symmetries in the leptonic sector [33,34, 88-90]. Here, we explore the intriguing possibility raised in Refs. [2, 25] that the stability of DM is due to the $\mathcal{Z}_{3}$ center group of the global $\mathcal{G}_{F}^{\mathrm{SM}} \equiv S U(3)_{Q} \times$ $S U(3)_{U} \times S U(3)_{D}$ quark flavor symmetry. While $\mathcal{G}_{F}^{\mathrm{SM}}$ is broken by the SM Yukawa interactions, its subgroup $\mathcal{Z}_{3}$ remains unbroken in the SM. More generally, it remains exact also in the presence of New Physics (NP), if the flavor breaking is of Minimally Flavor Violating (MFV) type, i.e. only due to the SM Yukawas. The lightest neutral state that is odd under $\mathcal{Z}_{3}$ is therefore stable and is a DM candidate. This is the idea behind MFV dark matter [2,23,25].

Requiring MFV is sufficient, but not necessary. In this chapter we formulate a general condition for flavored DM using flavor triality (see Eq. (3.3) below). For example, 
any spurion in the bifundamental of $\mathcal{G}_{F}^{\mathrm{SM}}$ leaves the above $\mathcal{Z}_{3}$ unbroken. The flavor breaking can thus be quite far from MFV and still have stability of DM guaranteed by the flavor dynamics. To illustrate this point we consider the model of Ref. [91] where the flavor-breaking spurions have the form $Y_{u, d}^{-1}$ and are thus canonically not of the MFV type. In this model the SM quark flavor symmetry $\mathcal{G}_{F}^{\mathrm{SM}}$ is fully gauged giving rise to flavor-gauge bosons, the Yukawas are promoted to physical scalar fields (flavons) transforming under flavor, and in addition there is a set of chiral fermions that cancel the anomalies in the flavor-gauge sector.

Using this renormalizable model we show below that a thermal relic DM can be in a nontrivial representation of $\mathcal{G}_{F}^{\mathrm{SM}}$. There are two conflicting constraints on this setup. On the one hand, Flavor Changing Neutral Current (FCNC) constraints impose lower bounds on the masses of states in nontrivial flavor representations. On the other hand, a DM relic density consistent with observations requires large enough DM annihilation cross section so that some of these same particles need to be sufficiently light. Both of these requirements are satisfied for $\mathcal{O}(\mathrm{TeV}) \mathrm{DM}$ mass. This is low enough that it may be tested by direct and indirect DM detection experiments and searched for at highenergy particle colliders.

While the phenomenology of flavored DM models can be found in Refs. [21,24$26,28,29,92-97]$, the construction of an explicit renormalizable model with inclusion of flavor-gauge interactions is new. Within our framework, the constraints on DM are more severe compared to a generic Effective Field Theory (EFT) analysis [2, 23]. In particular, the flavor constraints from new fermionic states, and the fact that the vacuum expectation values (vevs) of the flavon fields need to reproduce the quark masses, makes the structure of the theory much more rigid and predictive.

This chapter is structured as follows. In Sec. 3.3 we derive general conditions for DM to be stabilized by the exact accidental flavor symmetry of the SM (flavor triality). An explicit realization of this possibility is introduced in Sec. 3.3 in the form of a 
model with fully gauged $\mathcal{G}_{F}^{\mathrm{SM}}$. The resulting DM, flavor, and collider phenomenology is analyzed in detail in Sec. 3.4. We summarize our conclusions in Sec. 3.6, while more technical details of some of our computations are relegated to the Appendices.

\subsection{Stability of flavored dark matter}

We start by formulating the general conditions required for flavored DM to be stable due to flavor triality. The SM exhibits a large global flavor symmetry $U(3)_{Q} \times U(3)_{U} \times$ $U(3)_{D} \times U(3)_{L} \times U(3)_{E}$ in the limit of vanishing Yukawa interactions. In this chapter we focus on the quark sector. This has the global symmetry $\mathcal{G}_{F}^{\mathrm{SM}} \times U(1)_{Y} \times U(1)_{B} \times U(1)_{\mathrm{PQ}}$. The three $U(1)$ factors are the hypercharge, baryon number $(B)$, and the Peccei-Quinn symmetry, respectively. The remaining semisimple group is $\mathcal{G}_{F}^{\mathrm{SM}}=S U(3)_{Q} \times S U(3)_{U} \times$ $S U(3)_{D}$. The SM quarks transform under it as

$$
Q_{L} \sim(3,1,1), \quad U_{R} \sim(1,3,1), \quad D_{R} \sim(1,1,3) .
$$

The $\mathcal{G}_{F}^{\mathrm{SM}}$ global symmetry is broken by the SM Yukawa terms

$$
\mathcal{L}_{Y}=\bar{Q}_{L} \tilde{H} y_{u} U_{R}+\bar{Q}_{L} H y_{d} D_{R}+\text { h.c. },
$$

where $\tilde{H}=i \sigma_{2} H^{*} . \mathcal{L}_{Y}$ is formally invariant under $\mathcal{G}_{F}$ if $y_{u, d}$ are promoted to spurions that transform as $(3, \overline{3}, 1)$ and $(3,1, \overline{3})[63,64,66,67,98-100]$. NP is of MFV type if $y_{u, d}$ are the only flavor-breaking spurions also in the NP sector.

The SM Yukawa couplings in Eq. (3.2) break $U(1)_{\mathrm{PQ}}$ and break $\mathcal{G}_{F}^{\mathrm{SM}}$ to its center group $\mathcal{Z}_{3}^{U D Q}$, under which all three generations of quarks transform as

$$
\left\{U_{R}, D_{R}, Q_{L}\right\} \rightarrow e^{i 2 \pi / 3}\left\{U_{R}, D_{R}, Q_{L}\right\} \text {. In the SM the } \mathcal{Z}_{3}^{U D Q} \text { is identical to a subgroup }
$$


of $U(1)_{B}$. This is no longer true in the presence of NP. In MFV for instance, $\mathcal{Z}_{3}^{U D Q}$ remains exact, while $U(1)_{B}$ can be broken, e.g., by dimension-9 operators [101] (see also [25]).

The $\mathcal{Z}_{3}^{U D Q}$ may be the underlying reason for the stability of DM. To make this explicit it is useful to introduce $\mathcal{Z}_{3}^{\chi}$, a diagonal subgroup of $\mathcal{Z}_{3}^{U D Q} \times \mathcal{Z}_{3}^{c}$. Here, $\mathcal{Z}_{3}^{c}$ is the center group of color $S U(3)_{c}$, under which $\left\{U_{R}, D_{R}, Q_{L}\right\} \rightarrow e^{-i 2 \pi / 3}\left\{U_{R}, D_{R}, Q_{L}\right\}$. All the SM fields are thus $\mathcal{Z}_{3}^{\chi}$ singlets. In MFV NP $\mathcal{Z}_{3}^{\chi}$ is exact, so that the lightest $\mathcal{Z}_{3}^{\chi}$ odd particle is stable and can be a DM candidate [25].

We generalize this observation beyond MFV. To this end, we introduce the notion of flavor triality [25]. Consider a field $X$ in the $\mathcal{G}_{F}^{\mathrm{SM}}$ representation $X \sim\left(n_{Q}^{X}, m_{Q}^{X}\right) \times$ $\left(n_{U}^{X}, m_{U}^{X}\right) \times\left(n_{D}^{X}, m_{D}^{X}\right)$, where $n_{i}^{X}, m_{i}^{X}$ are the Dynkin coefficients of the corresponding $S U(3)_{i}$ group. We call flavor triality the quantity

$$
\left(n_{X}-m_{X}\right) \bmod 3
$$

where $n_{X}=n_{Q}^{X}+n_{U}^{X}+n_{D}^{X}$ and $m_{X}=m_{Q}^{X}+m_{U}^{X}+m_{D}^{X}$.

The basic requirements for flavored DM to be stable due to $\mathcal{Z}_{3}^{\chi}$ are the following. First of all, $\mathcal{G}_{F}^{\mathrm{SM}}$ needs to be a good symmetry in the UV. Secondly, $\mathcal{G}_{F}^{\mathrm{SM}}$ needs to be broken only by spurions $\langle\Phi\rangle$ with zero flavor triality, $\left(n_{\langle\Phi\rangle}-m_{\langle\Phi\rangle}\right) \bmod 3=0$. This ensures that $\mathcal{Z}_{3}^{\chi}$ is unbroken. (The spurions $\langle\Phi\rangle$ need to be color singlets in order not to break color.) The lightest $\mathcal{Z}_{3}^{\chi}$ odd state is then stable. If it is a color singlet it is a potential DM candidate. This also means that DM is in a nontrivial flavor representation with nonzero flavor triality, $\left(n_{\chi}-m_{\chi}\right) \bmod 3 \neq 0$.

The above shows that models with flavored DM can deviate significantly from MFV. In particular, $\mathcal{Z}_{3}^{\chi}$ is not broken by a vev of any field that is in an adjoint or in a bifundamental of $\mathcal{G}_{F}^{\mathrm{SM}}$. Specifically, any function $f\left(y_{u}, y_{d}\right)$ leaves $\mathcal{Z}_{3}^{\chi}$ unbroken. More generally, additional flavor-breaking sources that transform as $(8,1,1),(1,3, \overline{3}), \ldots$ may 
be present without spoiling DM stability. While the flavor structure of such NP models is not of MFV type in general, the stability of DM is still a consequence of an unbroken flavor subgroup. DM is in a nontrivial representation of the flavor group, leading to distinct phenomenology depending on the nature of the flavor breaking and on which flavor multiplet $\chi$ belongs to.

An important starting point in the above discussion was the assumption that $\mathcal{G}_{F}^{\mathrm{SM}}$ is a good symmetry in the UV. This is most easily achieved, if $\mathcal{G}_{F}^{S M}$ is gauged. We explore this possibility in the remainder of the chapter.

\subsection{Gauged flavor interactions and dark matter}

We gauge the full SM quark-flavor symmetry $\mathcal{G}_{F}^{\mathrm{SM}}$. The fermionic sector is extended to cancel the anomalies of the new gauge sector. We use the model of Ref. [91] that allows for $\mathcal{O}(\mathrm{TeV})$ flavor gauge bosons (FGBs). The SM Yukawas, $y_{u, d}$, arise from the vevs of new scalar fields transforming as

$$
Y_{u} \sim(\overline{3}, 3,1), \quad Y_{d} \sim(\overline{3}, 1,3),
$$

under $\mathcal{G}_{F}^{\mathrm{SM}}$. The minimal set of chiral fermions that ensures anomaly cancellation of the new gauged sector is

$$
\Psi_{u R} \sim(3,1,1), \quad \Psi_{d R} \sim(3,1,1), \quad \Psi_{u L} \sim(1,3,1), \quad \Psi_{d L} \sim(1,1,3),
$$

where the index $L$ and $R$ represents their chirality. Together with the SM fermions they, therefore, form vector-like representations of $\mathcal{G}_{F}^{\mathrm{SM}}$. The SM gauge quantum numbers of $\Psi_{u R}, \Psi_{d R}, \Psi_{u L}, \Psi_{d L}$ are the same as for $U_{R}, D_{R}, U_{R}, D_{R}$, respectively, i.e., they are $S U(2)_{L}$ singlets but charged under $U(1)_{Y}$. Because the new fermions are vector-like under the 
SM, e.g, $\Psi_{u R}$ transforms like $\Psi_{u L}$ under the SM, all anomalies in the SM sector cancel. Remarkably, with the above fermionic content also all mixed gauge anomalies cancel. In fact, one could also gauge two additional flavor diagonal $U(1)^{\prime} \mathrm{s}, U(1)_{U}$ and $U(1)_{D}$, as well as $U(1)_{B-L}$, a possibility that we do not pursue further, but is discussed in Ref. [91].

The Yukawa and relevant mass terms in the Lagrangian are [91]

$$
\begin{aligned}
\mathcal{L}_{\text {mass }} & \supset \lambda_{u} \bar{Q}_{L} \tilde{H} \Psi_{u R}+\lambda_{u}^{\prime} \bar{\Psi}_{u L} Y_{u} \Psi_{u R}+M_{u} \bar{\Psi}_{u L} U_{R} \\
& +\lambda_{d} \bar{Q}_{L} H \Psi_{d R}+\lambda_{d}^{\prime} \bar{\Psi}_{d L} Y_{d} \Psi_{d R}+M_{d} \bar{\Psi}_{d L} D_{R}+\text { h.c. },
\end{aligned}
$$

where $\lambda_{u, d}^{(\prime)}$ are flavor-universal coupling constants and $M_{u, d}$ flavor-universal mass parameters. The mass terms in Eq. (3.6) mix the states $\Psi_{u L, u R}$ and $U_{L, R}$ forming mass eigenstates $u_{i}$ and $u_{i}^{\prime}$, where $i=1,2,3$ is the generation index (and similarly for downquark states). After electroweak symmetry breaking the masses for the two masseigenstate sets are, in the limit $m_{u_{i}^{\prime}} \gg m_{u_{i}}$ [91, 102]

$$
m_{u^{i}} \approx \frac{v}{\sqrt{2}} \frac{\lambda_{u} M_{u}}{\lambda_{u}^{\prime}\left\langle Y_{u}\right\rangle_{i}}, \quad m_{u_{i}^{\prime}} \approx \lambda_{u}^{\prime}\left\langle Y_{u}\right\rangle_{i}
$$

The mass matrix for the FGBs, $A_{A}^{a}, A=Q, U, D$ and $a=1, \ldots, 8$, is governed by the vevs of the $Y_{u, d}$ scalar fields and the gauge couplings, [91,102]

$$
\left(\mathcal{M}_{A B}^{2}\right)_{a b}=\frac{1}{4} g_{A} g_{B} \operatorname{Tr}\left[\left\langle Y_{u}\right\rangle\left\{\lambda^{a}, \lambda^{b}\right\}\left\langle Y_{u}\right\rangle^{\dagger}\right]\left(\delta_{A B} \delta_{A Q}-2 \delta_{A Q} \delta_{B U}+Q \leftrightarrow U\right)+U, u \leftrightarrow D, d,
$$

with $\lambda^{a}$ the Gell-Mann $S U(3)$ matrices. The mass matrix is $24 \times 24$ dimensional. We denote the mass-ordered eigenstates by $A^{m}, m=1, \ldots, 24$, where $A^{24}$ is the lightest one. The lightest gauge boson is found to be along the three diagonal $\lambda_{8}$ directions. This is a consequence of $\left\langle Y_{u}\right\rangle$ and $\left\langle Y_{d}\right\rangle$ being almost aligned and with very hierarchical eigenvalues, where the $\left\langle Y_{u}\right\rangle_{33}$ and $\left\langle Y_{d}\right\rangle_{33}$ are the smallest eigenvalues. 
The SM Yukawas, $y_{u, d}$, are generated after $Y_{u, d}$ obtain a vev and the $\Psi_{i}$ fields are integrated out. To first order in $M_{u, d} /\left\langle Y_{u, d}\right\rangle$, this gives

$$
y_{u} \simeq \frac{\lambda_{u} M_{u}}{\lambda_{u}^{\prime}\left\langle Y_{u}\right\rangle}, \quad y_{d} \simeq \frac{\lambda_{d} M_{d}}{\lambda_{d}^{\prime}\left\langle Y_{d}\right\rangle}
$$

The SM Yukawas, $y_{u, d}$, are non-analytic functions of the spurions $\left\langle Y_{u, d}\right\rangle$, which signals that the theory is strictly speaking not MFV. Analogously, the NP states, $u_{i}^{\prime}, d_{i}^{\prime}$ and $A^{m}$, have masses that are non-analytic in terms of the SM Yukawas. However, the lowenergy observables, with only the SM fields on the external legs can be MFV-like. If the $M_{u, d} /\left\langle Y_{u, d}\right\rangle$ suppressed terms are kept in Eq. (3.9), the $y_{u, d}$ become more involved functions of $\left\langle Y_{u, d}\right\rangle^{-1}$. These are analytic in $\left\langle Y_{u, d}\right\rangle^{-1}$ since the effects of NP states decouple in the $\left\langle Y_{u, d}\right\rangle \rightarrow \infty$ limit. Similarly, the NP contributions to the low-energy observables $C_{i}$ take the form $\delta C_{i}=F\left(\left\langle Y_{u}\right\rangle^{-1},\left\langle Y_{d}\right\rangle^{-1}\right)=\tilde{F}\left(y_{u}, y_{d}\right)$, with $F, \tilde{F}$ analytic functions. One can thus expand $\delta C_{i}=a_{1} y_{u} y_{u}^{\dagger}+a_{2}\left(y_{u} y_{u}^{\dagger}\right)^{2}+b_{1} y_{u} y_{u}^{\dagger} y_{d} y_{d}^{\dagger}+\cdots$, where we assumed for illustration that the transition is due to the left-handed quark current. As long as there are no large flavor-conserving ratios, i.e., as long as $\left(\lambda_{u} M_{u} / \lambda_{u}^{\prime}\right) /\left(\lambda_{d} M_{d} / \lambda_{d}^{\prime}\right) \ll 1 /\left|V_{c b}\right|$, the Taylor expansion can be truncated after a few terms (see Ref. [100] for a more detailed discussion). In this limit, the low-energy effects are of the MFV type, suppressing the FCNCs to acceptable levels already for NP states at the electroweak scale. In a numerical analysis that we perform in Appendix B.I, we find that the expansion of the effective weak Hamiltonian in terms of SM Yukawa couplings can indeed still be performed for FGB contributions.

Since $\left\langle Y_{u, d}\right\rangle$ are in the bifundamental representation of $\mathcal{G}_{F}^{\mathrm{SM}}$, the $\mathcal{Z}_{3}^{\chi}$ remains unbroken. As argued above the $\mathcal{Z}_{3}^{\chi}$ can be used to make flavored DM candidates stable. We consider two examples: i) a fermionic DM in a vector-like representation of $\mathcal{G}_{F}^{\mathrm{SM}}$ that thermalizes with the visible sector through FGBs, and ii) a scalar flavored DM, that interacts with the visible sector by exchanging FGBs as well as the Higgs. 


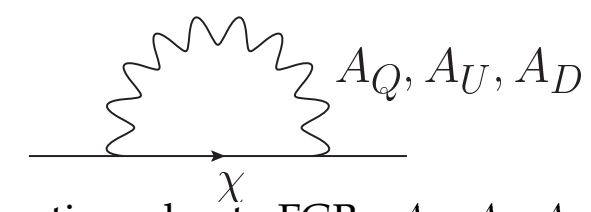

Figure 3.1: Radiative corrections due to FGBs, $A_{Q}, A_{u}, A_{D}$, split the DM multiplet $\chi$.

\subsubsection{Fermionic flavored dark matter}

The DM in the first model is a massive Dirac fermion, $\chi$, in a vector-like representation of $S U(3)_{U}$,

$$
\chi \sim(1,3,1)
$$

so that no gauge anomalies are induced. Its mass term is

$$
\mathcal{L}_{\text {mass }}^{\chi}=m_{\chi}^{0} \bar{\chi} \chi
$$

Since $\chi$ is charged under $\mathcal{Z}_{3}^{\chi}$, the lightest member of the $\chi$ triplet is stable. Note that we could also gauge a larger global group $\mathcal{G}_{F}^{S M} \times S U(3)_{\chi}$, with $\chi$ transforming under $S U(3)_{\chi}$. That we identify $S U(3)_{\chi}$ with $S U(3)_{U}$ is a model-building choice.

The DM flavor triplet, $\chi$, is split by radiative corrections due to the exchanges of FGBs, see Fig. 3.1. In the $m_{\chi}^{0} \ll m_{A}$ limit, the DM mass splitting at one-loop is given by

$$
\Delta m_{\chi}=-\frac{3}{4} \frac{g_{U}^{2}}{16 \pi^{2}} m_{\chi}^{0}\left(\Xi-\frac{1}{3} \operatorname{Tr} \Xi\right)
$$

where $\Delta m_{\chi}$ is a $3 \times 3$ matrix and so is $\Xi \equiv \lambda^{a}\left(\log \mathcal{M}_{A}^{2} / \mu^{2}\right)^{a b} \lambda^{b}$. The FGB mass matrix $\mathcal{M}_{A}^{2}$ is given in Eq. (3.8), while the $a, b$ indices run only over the eight $S U(3)_{U}$ generators. The unphysical $\mu$ dependence cancels in the r.h.s. of Eq. (3.12). The $\chi_{i}, i=1,2,3$, mass eigenstates are obtained by diagonalizing the mass matrix $\Delta m_{\chi}$. And $\Xi$ is a function of $Y_{u}^{\dagger} Y_{u}$ and $Y_{u}^{\dagger} Y_{d} Y_{d}^{\dagger} Y_{u}$ vev combinations, making the $\chi$ mass eigen-basis slightly misaligned with respect to the up-quark one. The $\chi_{1}$ mass receives contributions from the 


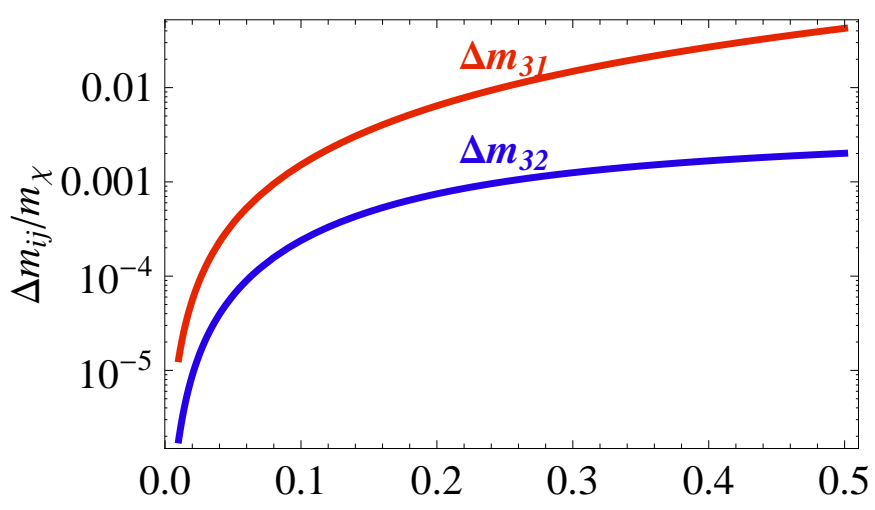

Figure 3.2: Typical radiative splitting of the ferifhionic DM multiplet with $\Delta m_{31}\left(\Delta m_{32}\right)$ shown in red (blue) as a function of $g_{U}$, while all other parameters are kept fixed at $g_{Q}=0.4, g_{D}=0.5, M_{u}=600 \mathrm{GeV}, M_{d}=400 \mathrm{GeV}, \lambda_{u}=1, \lambda_{u}^{\prime}=0.5, \lambda_{d}=0.25, \lambda_{d}^{\prime}=0.3$.

heaviest FGBs (cf. section 3.5). The lightest state is thus $\chi_{1}$, i.e., with the predominantly up-quark flavor, while the heaviest is the top-flavored state, $\chi_{3}$.

In the numerics we use the exact one-loop expressions for the DM mass splitting,

$$
\Xi=\frac{3}{2} \lambda^{a} \mathcal{W}^{a+8, m}\left[B_{1}\left(m_{\chi}^{2}, m_{A_{m}}^{2}, m_{\chi}^{2}\right)-B_{0}\left(m_{\chi}^{2}, m_{A_{m}}^{2}, m_{\chi}^{2}\right)\right] \mathcal{W}^{\dagger^{m, c+8}} \lambda^{c} .
$$

Summation over FGB mass eigenstate indices $m=1, \ldots, 24$ and over $a, c=1, \ldots, 8$ is understood. The $24 \times 24$ dimensional matrix $\mathcal{W}$ diagonalizes the gauge-boson mass matrix and $B_{0,1}$ are the Veltman-Passarino functions. Typical values of the mass splitting as a function of $g_{U}$ are shown in Fig. 3.2. Denoting $\Delta m_{i j} \equiv m_{\chi_{i}}-m_{\chi_{j}}$, we see that $\Delta m_{32} \ll \Delta m_{31}$, so that the lightest state $\chi_{1}$ is split away from $\chi_{2}$ and $\chi_{3}$, with the latter approximately degenerate, $m_{\chi_{2}} \simeq m_{\chi_{3}}$. This is very different from MFV DM $[2,23,25]$ where the DM mass splitting is assumed to be expandable in the SM Yukawa couplings. In that case one has an approximate $U(2)$ symmetry for the first two generations giving $m_{\chi_{1}} \simeq m_{\chi_{2}}$, while the top-flavored DM, $\chi_{3}$, is split away from the first two generations, and can be either significantly heavier or lighter.

The relation $\Delta m_{32} \ll \Delta m_{31}$ signifies that our flavored DM is non-MFV. The flavor 
gauge group $S U(3)_{U}$ is broken by the FGB vevs $\left\langle Y_{u}\right\rangle$. This breaking is larger in the first two generations. Since the quark masses are inversely proportional to $\left\langle Y_{u}\right\rangle$, this leads to an approximate global $U(2)_{U}$ symmetry in the quark sector. Such an approximate symmetry is not found for the radiative corrections to DM masses, $m_{\chi_{i}}$. The DM multiplet has a chiral symmetry in the limit $m_{\chi} \rightarrow 0$ ensuring that the radiative corrections are proportional to $m_{\chi}$ and only log-dependent on FGB masses. The splitting does not vanish in the $\left\langle Y_{u}\right\rangle \rightarrow \infty$ limit (or, equivalently, $y_{u} \rightarrow 0$ limit), since in this limit the $S U(3)_{U}$ gauge group is still completely broken. Numerically, for $m_{\chi} \sim \mathrm{TeV}$ the splittings are $\Delta m_{31} \sim \mathcal{O}(10 \mathrm{GeV})$ and $\Delta m_{32} \sim \mathcal{O}(1 \mathrm{GeV})$ for $g_{U} \sim 0.4$ and can be less than a pion mass for an order of magnitude smaller $g_{U}$.

The DM multiplet can be split more significantly if there is flavor breaking beyond $\left\langle Y_{u}\right\rangle,\left\langle Y_{d}\right\rangle$. As an example we consider an additional scalar field in the adjoint of $S U(3)_{U}, \Phi_{U} \sim(1,8,1)$. The DM mass Lagrangian now reads

$$
\mathcal{L}_{\text {mass }}^{\prime}=m_{\chi}^{0} \bar{\chi}_{L} \chi_{R}+\lambda_{\chi} \bar{\chi}_{L} \Phi_{U} \chi_{R}+\text { h.c. },
$$

and yields DM masses that are split already at tree level, $\Delta m_{\chi}=\lambda_{\chi}\left\langle\Phi_{U}\right\rangle$. We assume that $\left\langle\Phi_{U}\right\rangle$ is aligned with $Y_{U}^{\dagger} Y_{U}$. Then the two diagonalize in the same basis giving $\mathcal{O}(1)$ splitting between all three members of the multiplet. The alignment is not needed in general, but does simplify our analysis. For the same reason, we also take the first state to be the lightest one, $m_{\chi_{1}}<m_{\chi_{2,3}}$.

The $\chi_{i}$ interact with the SM through FGBs. This also induces the decay of the heavier two states in the DM multiplet, $\chi_{2,3}$, to $\chi_{1}$. We parametrize the relevant interactions 
with

$$
\begin{aligned}
\mathcal{L}_{\chi} & \supset\left(\hat{g}_{\chi}^{m}\right)_{i j} \bar{\chi}_{i} \gamma^{\mu} \chi_{j} A_{\mu}^{m} \\
& +\bar{u}_{k} \gamma^{\mu}\left(\left(\hat{\mathcal{G}}_{R}^{u}\right)_{k l, m} P_{R}+\left(\hat{\mathcal{G}}_{L}^{u}\right)_{k l, m} P_{L}\right) u_{l} A_{\mu}^{m} \\
& +\bar{d}_{k} \gamma^{\mu}\left(\left(\hat{\mathcal{G}}_{R}^{d}\right)_{k l, m} P_{R}+\left(\hat{\mathcal{G}}_{L}^{d}\right)_{k l, m} P_{L}\right) d_{l} A_{\mu}^{m},
\end{aligned}
$$

where $P_{R, L} \equiv \frac{1}{2}\left(1 \pm \gamma^{5}\right)$ and $k, l=1, \ldots, 6$. The couplings of $\chi_{i}$ to the gauge bosons are

$$
\left(\hat{g}_{\chi}^{m}\right)_{j i}=\left(-\frac{1}{2} g_{U} U^{\dagger} \lambda_{n}^{U} \mathcal{W}^{n m} U\right)_{j i}
$$

where $U$ diagonalizes the $m_{\chi}$ mass matrix, $U^{\dagger} m_{\chi} U=\hat{m}_{\chi}$, and $\mathcal{W}$ diagonalizes the gauge-boson mass matrix. The explicit form of FGB couplings to exotic and SM quarks, $\left(\hat{\mathcal{G}}_{L / R}^{u / d}\right)_{k l, m}$, can be found in Appendix A.2 of Ref. [102].

The partial decay width for $\chi_{i} \rightarrow \chi_{j} q_{l} \bar{q}_{k}$ is, neglecting hadronization effects,

$$
\Gamma\left(\chi_{i} \rightarrow \chi_{j} q_{l} \bar{q}_{k}\right)=\frac{3}{(2 \pi)^{3}} \frac{\Delta m_{i j}^{5}}{15}\left[\left|\sum_{m}\left(\hat{g}_{\chi}^{m}\right)_{i j} \frac{1}{m_{A^{m}}^{2}}\left(\hat{\mathcal{G}}_{L}^{u}\right)_{k l, m}\right|^{2}+L \rightarrow R\right]
$$

where the sum runs over the FGB mass eigenstates $m=1, \ldots, 24$. Expression (3.17) is valid in the $\left|\Delta m_{i j}\right| \ll m_{\chi_{i}}$ limit, and neglecting the quark masses. The above approximations are valid for all the values of parameter for which the correct relic abundance is obtained and the FCNC, collider and direct DM detection constraints are satisfied.

If the mass splitting is less than the pion mass the decay $\chi_{i} \rightarrow \chi_{j} q_{l} \bar{q}_{k}$ is kinematically not allowed. The heavier $\chi_{i}$ states then decay radiatively to $\chi_{i} \rightarrow \chi_{j} \gamma \gamma$. For our purposes an order of magnitude estimate of the decay width suffices. The naive 
dimensional analysis estimate gives

$\Gamma\left(\chi_{i} \rightarrow \chi_{j} \gamma \gamma\right) \sim \frac{\Delta m_{i j}^{9}}{8 \pi} \frac{1}{16 \pi^{2}}\left(\frac{\alpha}{4 \pi}\right)^{2}\left[\left|\sum_{m, f} \frac{\left(\hat{g}_{\chi}^{m}\right)_{i j}}{m_{A^{m}}^{2}} \frac{Q_{u}^{2}}{m_{f}^{2}}\left(\left(\hat{\mathcal{G}}_{L}^{u}\right)_{f f, m}-\left(\hat{\mathcal{G}}_{R}^{u}\right)_{f f, m}\right)\right|^{2}+u \rightarrow d\right]$

where $Q_{u}=2 / 3$ and $Q_{d}=-1 / 3$ are the electromagnetic charges of up and down quarks. The sum over $m$ runs over the FGB mass eigenstates, while the sum over $f$ is

over the SM quarks and exotic states, of mass $m_{f}$ (for up, down and strange quarks this needs to be replaced with $\left.\Lambda_{\mathrm{QCD}}\right)$.

\subsubsection{Scalar flavored dark matter}

The second model has scalar DM, $\phi$, in a fundamental representation of $S U(3)_{U}$

$$
\phi \sim(1,3,1)
$$

The main difference with the fermionic flavored DM from the previous subsection is that the scalar DM interacts with the visible sector via two different types of interactions. The first is its couplings to the FGBs, which is similar to the case of the fermionic DM. The second is a direct coupling to the Higgs

$$
\mathcal{L}_{\text {int }}^{\mathrm{DM}}=\lambda_{H}\left(\phi^{\dagger} \phi\right)\left(H^{\dagger} H\right)
$$

For a thermal relic the DM annihilations proceed predominantly through the Higgs portal. The interactions via FGBs are subdominant except if $m_{\phi} \simeq m_{A}^{a} / 2$ for some $A^{a}$. Unless this is the case, the fact that the DM carries a flavor quantum number is exhibited only through the multiplicity of the states. 
After electroweak symmetry breaking, the DM-Higgs interactions are given by

$$
\mathcal{L}_{\text {int }}^{\mathrm{DM}} \supset \lambda_{H}\left(v h+v^{2} / 2\right) \phi^{\dagger} \phi,
$$

and the DM mass term $m_{0}^{2} \phi^{\dagger} \phi$ is shifted by the Higgs condensate to give the DM mass of

$$
m_{\phi}^{2}=m_{0}^{2}+v^{2} / 2 .
$$

The vevs of the flavons, $\left\langle Y_{u}\right\rangle$ and $\left\langle Y_{d}\right\rangle$, split the DM multiplet at tree level through

$$
\mathcal{L} \supset \kappa_{1}\left(\phi^{\dagger} \lambda^{a} \phi\right) \operatorname{Tr}\left(Y_{u}^{\dagger} \lambda^{a} Y_{u}\right)+\kappa_{2}\left(\phi^{\dagger}\left\{\lambda^{a}, \lambda^{b}\right\} \phi\right) \operatorname{Tr}\left(Y_{u}^{\dagger}\left\{\lambda^{a}, \lambda^{b}\right\} Y_{u}\right) .
$$

The spectrum is also split by radiative corrections due to FGBs. These are quadratically divergent and proportional to the square of the FGB mass. In principle, it is possible to fine tune the tree-level and loop contributions to give almost degenerate DM flavor multiplet. However, given the hierarchical FGB masses, it is more likely that the DM multiplet is split completely, and only the lightest state is relevant for DM phenomenology. Depending on the signs of $\kappa_{i}$ in Eq. (3.23) the lightest $\phi$ component can be either top-quark or up-quark flavored. We choose the latter option in the numerics for easier comparison with the fermionic case.

\subsection{Dark matter and new physics phenomenology}

We turn next to the phenomenology of the flavored DM models. We perform a scan over the parameters of the models and show that the lowest DM states, both for the fermionic DM, $\chi$, and the scalar DM, $\phi$, can be thermal relics. To make the scan numerically tractable we rely on several approximations in calculating the relic density, which we explain below. We also discuss the predictions for direct DM detection, and 
the constraints from FCNCs and collider searches.

\subsubsection{Scan results}

In the scan we fix $\lambda_{u}=1$ and vary $\lambda_{d} \in[1 /(4 \pi), 1]$. The range is chosen with the expectation that one will be able to accommodate both the SM top and bottom quark Yukawas as well as satisfy electroweak precision constraints and direct $t^{\prime}$ and $b^{\prime}$ searches [91]. In addition we vary conservatively $\lambda_{u, d}^{\prime} \in\left[1 /(4 \pi)^{2}, 4 \pi\right], g_{Q, U, D} \in\left[1 /(4 \pi)^{2}, 4 \pi\right]$, and $M_{u, d} \in[0.2,20] \mathrm{TeV}$. To a good approximation, the variation of $M_{u}$ effectively compensates the fact that we do not vary $\lambda_{u}$ as seen from Eq. (3.9). We have verified that further extending these parameter ranges does not extend the viable DM-model parameter space. For instance, the upper ranges of $g_{Q, U, D}$ and $\lambda_{u, d}^{\prime}$ already lie in the nonperturbative regime. To ensure perturbative control we require that all the FGB decay widths satisfy $\Gamma_{A^{m}}<0.5 m_{A^{m}}$, and that the radiative mass splitting for the fermionic $\mathrm{DM}$ is $\left|\Delta m_{i j}\right|<0.5 m_{\chi}$. This imposes upper bounds on $g_{Q, U, D}$ that are typically close to $\sqrt{4 \pi}$. Similar constraints on $\lambda_{u, d}^{\prime}$ are expected to follow from analogous considerations in the flavored Higgs sector, i.e., by requiring the total widths of the flavored scalars $Y_{u, d}$ to be small compared to their masses.

The results of the scan are shown in Figs. 3.3, 3.4, 3.5. Fig. 3.3 (upper panels) show the results of the scan for fermionic DM model with radiative (left) and large treelevel mass splitting (right). Fig. 3.3 (lower panel) instead shows the results of the scan for scalar DM. All the points shown in Fig. 3.3 give the correct relic DM abundance, $\Omega_{\mathrm{DM}}$. Different colors denote consecutively applied constraints. The grey points fail the perturbativity requirement, $\Gamma_{A^{m}}<0.5 m_{A^{m}},\left|\Delta m_{i j}\right|<0.5 m_{\chi}$ discussed above. The points in brown are excluded by direct DM detection, the points in dark magenta by $t^{\prime}$ direct searches and the points in orange by dijet resonance searches. The flavor bounds

exclude points in light magenta, while cosmological considerations - mainly from big 
Figúre 3.3: The resplitursedich the scan for fermionic DM with radiative mass splitting (upper left panథd), in panel) flefored DM Ceonstraints from perturbativity (grey), $t^{\prime}$ (dark magenta) and dijet reson (blue) and direct DM detetion (brown) are consecutively applied. Allowed parameter points are glenoted by green. For scalar flavor DM (right) we show the LUX and perturbativity bounds as two grey bands. The four benchmark points for fermionic

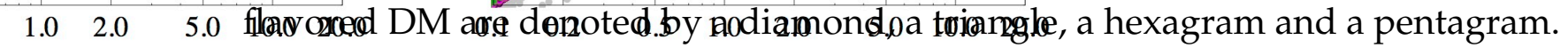

$$
m_{\chi_{1}}[\mathrm{TeV}] \quad m_{\chi_{1}}[\mathrm{TeV}]
$$

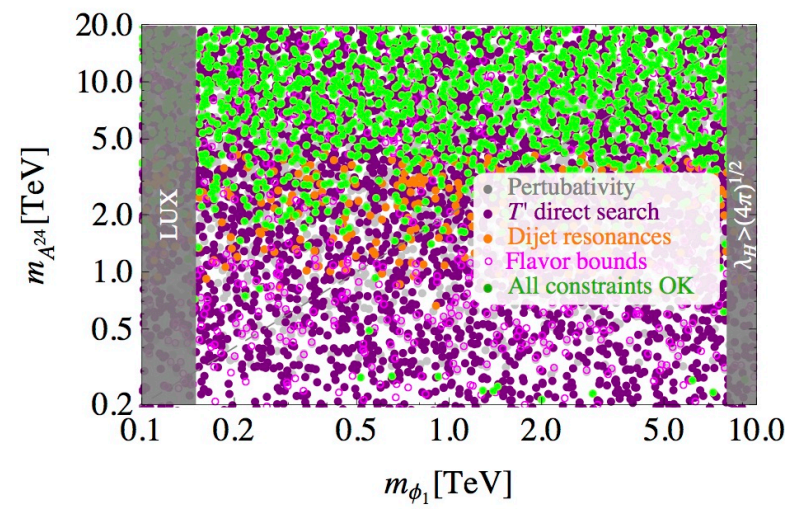



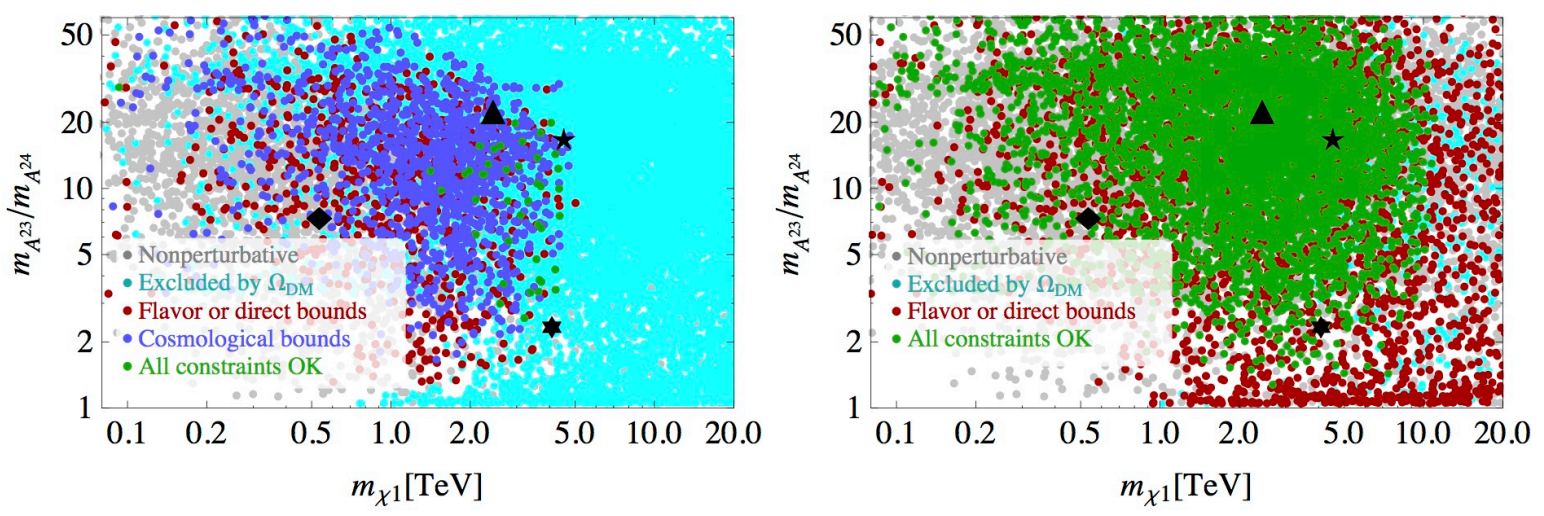

Figure 3.4: The ratio of masses of the next-to-lightest to the lightest FGBs, $m_{A^{23}} / m_{A^{24}}$ for radiatively split DM multiplet (upper left panel), and for the large mass splitting limit (upper right panel), as functions of the DM mass, $m_{\chi_{1}}$, for the fermionic flavored DM. Lower panel shows the relative radiative mass splitting in the DM multiplet. The constraints due to perturbativity (grey), too large relic abundance (light blue), early cosmology (dark blue), flavor and direct bounds (dark red), are applied consecutively, leaving allowed points (green).

bang nucleosynthesis - exclude points in dark blue. The green points are allowed by all constraints. In Figs. 3.4 and 3.5 we also show the points where it is not possible to obtain the.çorrect relic abundance (denoted by light blue), and denote by dark red the

₹ 0.010 Nonperturbative For fermionic DM the observed relic abundance requires resonantly enhanced an$0.005 \because$ Flavor or direct bounds 0.001

$\because$ Cosmolosicthithation through $s$-channel exchange of the lightest FGB, $A^{24}$, see Fig. 3.6 (left). This

$0.1 \quad 0.2$ ledefs to the corréfation $\pi_{\chi_{i}}^{0.0} \simeq m_{A^{24}} / 2$ shown in Fig. 3.3 (upper panels). It is possible $m_{\chi 1}[\mathrm{TeV}]$

to obtain the correct relic abundance also if the DM mass is only approximately half 
Figure 3.5: The maximal decay time of the two heavy states in the DM multiplet as functions of DM mass (left) and the minimal mass splitting in the DM multiplet (right) for radiatively split fermionic flavored DM. The color coding is as in Fig. 3.4.

of the lightest FGB (points away from the diagonal in Fig. 3.3 (upper panels)). These points require at least some of the couplings to be large and are excluded by flavor, collider, direct detection, or perturbativity constraints. For the allowed points the relation $m_{\chi_{i}} \simeq m_{A^{24}} / 2$ is satisfied to within a few decay widths of $A^{24}$, i.e. to within $\mathcal{O}(10 \%)$. The scalar flavored DM, $10^{1}$ on the other hand, predominantly annihilates through the

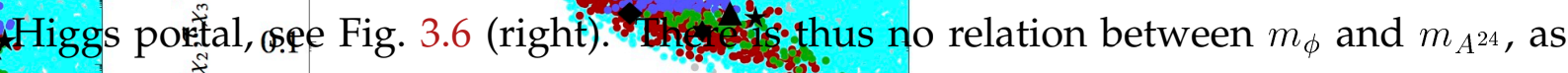

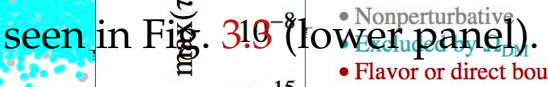

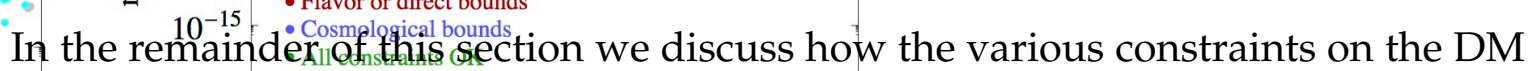
$10^{-22}$

5mođle. 6 were obtaihed. $1 \quad 10 \quad 100 \quad 1000$ $\min \left(\Delta m_{21}, \Delta m_{31}\right)[\mathrm{GeV}]$

\subsubsection{Thermal relic}

For the calculation of the DM relic density we follow Refs. [103, 104]. To speed-up the numerical scan we work in the non-relativistic limit, using the freeze-out approximation. The details of the calculation are given in Appendix B.II. Among viable parameter points we choose four benchmarks that satisfy all other experimental constraints. For the benchmark points we verify the DM relic abundance calculation using the MadDM [105] package. We computed the required Feynman rules using the Feyn- 

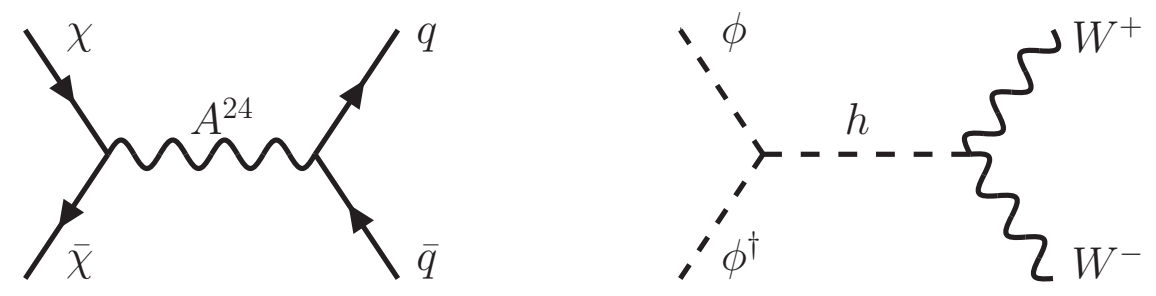

Figure 3.6: The Feynman diagrams for the dominant processes in the DM annihilation for fermionic (left) and scalar (right) flavored DM. For scalar DM only one representative diagram is shown; other relevant final states include $b \bar{b}, c \bar{c}, \tau \tau$ and $t \bar{t}, h h, Z Z$ (when kinematically allowed).

rules [106] package.

\subsubsection{Fermionic dark matter}

In the fermionic DM model the DM annihilation to quarks is dominated by s-channel exchange of the lightest FGB, $A^{24}$, see Fig. 3.6 (left panel). The $\chi_{i} \bar{\chi}_{i} \rightarrow \bar{u}_{j} u_{j}$ annihilation cross section is given by

$$
\sigma\left(\chi_{i} \bar{\chi}_{i} \rightarrow \bar{u}_{j} u_{j}\right) \simeq \frac{\left(\hat{g}_{\chi}^{24}\right)_{i i}^{2}}{4 \pi} \frac{s^{1 / 2}\left(s+2 m_{\chi_{i}}^{2}\right)}{\sqrt{s-4 m_{\chi_{i}}^{2}}} \frac{\left(\hat{\mathcal{G}}_{V}^{u}\right)_{j j, 24}^{2}+\left(\hat{\mathcal{G}}_{A}^{u}\right)_{j j, 24}^{2}}{\left(s-m_{A^{24}}^{2}\right)^{2}+m_{A^{24}}^{2} \Gamma_{A^{24}}^{2}}
$$

where

$$
\hat{\mathcal{G}}_{V, A}^{u}=\left(\hat{\mathcal{G}}_{L}^{u} \pm \hat{\mathcal{G}}_{R}^{u}\right) / 2,
$$

$\sqrt{s}$ is the center of mass energy and $\Gamma_{A^{24}}, m_{A^{24}}$ are the decay width and mass of the lightest FGB, respectively. In Eq. (3.24) we have neglected quark masses; the full expression is given in Eq. (B.14). The $\chi_{i} \bar{\chi}_{i} \rightarrow \bar{d}_{j} d_{j}$ annihilation cross section follows from Eq. (3.24) by replacing $u \rightarrow d$. The full decay width of the lightest FGB is the sum of all partial decay widths for kinematically allowed channels,

$$
\Gamma\left(A^{24} \rightarrow \bar{u}_{j} u_{j}\right) \simeq \frac{m_{A}^{24}}{4 \pi}\left(\left(\hat{\mathcal{G}}_{V}^{u}\right)_{j j, 24}^{2}+\left(\hat{\mathcal{G}}_{A}^{u}\right)_{j j, 24}^{2}\right) .
$$


In the above expression we have neglected the quark masses for simplicity, with the full expression given in Eq. (B.15). The rates for $A^{24} \rightarrow \chi_{i} \bar{\chi}_{i}, \bar{d}_{j} d_{j}$ are obtained by trivial coupling replacements and by correcting the color multiplicity factors.

The correct relic abundance requires resonant annihilation, $m_{\chi} \simeq m_{A^{24}} / 2$, see Fig. 3.3 (upper panels). This implies an upper bound on the DM mass through the following argument. The thermally averaged DM annihilation cross section scales in the narrow width approximation as

$$
\langle\sigma v\rangle \propto \frac{g_{A^{24}}^{4}}{m_{A^{24}} \Gamma_{A^{24}}}+\mathcal{O}\left(\Gamma_{A^{24}} / m_{A^{24}}\right) \sim \frac{1}{\langle Y\rangle_{A^{24}}^{2}} .
$$

Here, we used the approximate scaling for the FGB masses and decay widths, $m_{A^{24}} \sim$ $\langle Y\rangle_{A^{24}} g_{A^{24}}, \Gamma_{A^{24}} \sim\left(g_{A^{24}}\right)^{2} m_{A^{24}}$. The $\langle Y\rangle_{A^{24}}$ and $g_{A^{24}}$ are, respectively, the projections of the $Y_{u, d}$ vevs and $g_{Q, U, D}$ couplings onto the lightest FGB, $A^{24}$. The DM relic abundance is $\Omega_{\mathrm{DM}} \propto 1 /\langle\sigma v\rangle \propto\langle Y\rangle_{A^{24}}^{2}$ and thus depends predominantly only on the flavon vevs. Not exceeding the relic abundance puts an upper bound $\langle Y\rangle_{A^{24}} \lesssim \mathcal{O}($ few $100 \mathrm{GeV})$, almost independent of the DM mass. Since $m_{A^{24}} \sim\langle Y\rangle_{A^{24}} g_{A^{24}}$, and $g_{A^{24}} \lesssim \sqrt{4 \pi}$ for the theory to be perturbative, this also sets an upper bound on the lightest FGB mass. This in turn implies an upper bound on the DM mass through the relation $m_{\chi} \simeq m_{A^{24}} / 2$.

In the limit where only $\chi_{1}$ contributes to the DM relic abundance we find, using the scan, an upper bound $m_{\chi_{1}} \lesssim 10 \mathrm{TeV}$. The approximation is valid if $\chi_{2,3}$ states decay well before $\chi_{1}$ freezes out (i.e. $\tau_{\chi_{2,3}} \lesssim 10^{-11} \mathrm{~s}$ for $m_{\chi} \sim 1 \mathrm{TeV}$ ). For purely radiative DM mass splitting this is never the case (c.f. Fig. 3.5). Instead, if $\chi_{2,3}$ decay after decoupling, they will also contribute to the final DM relic abundance and one needs to sum all three contributions. In this case, the constraints on the mass spectrum become much more severe. In particular, in order for all $\chi$ components to annihilate efficiently their masses need to be within a few decay widths away from the lightest FGB (LFGB) mass. This in turn implies that the (radiative) DM mass splitting has to be of the order of the 
LFGB width. Since the splitting increases with $g_{U}$ we expect these effects to decrease the effective thermal DM annihilation cross section much before the theory becomes non-perturbative. Indeed we find, using the scan, an upper bound $m_{\chi_{1}} \lesssim 5 \mathrm{TeV}$.

Fig. 3.4 (upper panels) shows the ratio of masses of the next-to-lightest and the lightest FGB, $m_{A^{23}} / m_{A^{24}}$, as a function of DM mass $m_{\chi_{1}}$ for radiatively split DM multiplet (left) and in the large mass splitting limit (right). In most of the parameter space satisfying the $\Omega_{\mathrm{DM}}$ constraint $A^{23}$ is much heavier than $A^{24}$ so that the effects of higher resonances are indeed negligible. This justifies the use of only the lightest FGB when calculating the DM density in the scan.

Fig. 3.4 (lower panel) shows the relative radiative mass splitting $\Delta m_{21} / m_{\chi_{1}}$ and $\Delta m_{31} / m_{\chi_{1}}$ as a function of the DM mass, $m_{\chi_{1}}$ (both splittings are shown in one plot). In most cases the mass splitting is below $\mathcal{O}(10 \%)$ in order for all $\chi$ components to lie close to the resonant condition, as anticipated.

\subsubsection{Scalar dark matter}

For scalar DM the interactions with the visible sector are mainly due to the Higgsportal operator, $\lambda_{H}\left(\phi^{\dagger} \phi\right)\left(H^{\dagger} H\right)$, in Eq. (3.20). The interactions due to the exchanges of FGBs are subleading except for the resonant annihilation regions $m_{\phi_{1}} \simeq m_{A^{24}} / 2$. By adjusting the value of $\lambda_{H}$ one can obtain the correct relic abundance for any mass of $m_{\phi_{1}}$ irrespective of the lightest FGB mass, $m_{A^{24}}$, see Fig. 3.3 (bottom panel). In the calculation of the thermal relic abundance we include the following annihilation channels: $\phi_{1}^{\dagger} \phi_{1} \rightarrow \bar{b} b, \bar{c} c, \tau^{+} \tau^{-}, W^{+} W^{-}, Z Z, h h$ and $\bar{t} t$, see Fig. 3.6. The annihilation cross 
sections are

$$
\begin{gathered}
\sigma\left(\phi_{1}^{\dagger} \phi_{1} \rightarrow \bar{f} f\right)=\frac{\lambda_{H}^{2} m_{f}^{2} N_{c}\left(1-4 m_{f}^{2} / s\right)^{3 / 2}}{8 \pi \sqrt{1-4 m_{\phi_{1}}^{2} / s}\left[\left(m_{h}^{2}-s\right)^{2}+m_{h}^{2} \Gamma_{h}^{2}\right]}, \\
\sigma\left(\phi_{1}^{\dagger} \phi_{1} \rightarrow V V\right)=\frac{c_{V} \lambda_{H}^{2}}{16 \pi s} \frac{\sqrt{1-4 m_{V}^{2} / s}\left(12 m_{V}^{4}-4 m_{V}^{2} s+s^{2}\right)}{\sqrt{1-4 m_{\phi_{1}}^{2} / s}\left[\left(m_{h}^{2}-s\right)^{2}+m_{h}^{2} \Gamma_{h}^{2}\right]},
\end{gathered}
$$

where $c_{W}=1, c_{Z}=1 / 2$ and

$$
\sigma\left(\phi_{1}^{\dagger} \phi_{1} \rightarrow h h\right)=\frac{\lambda_{H}^{2}}{32 \pi s} \frac{\sqrt{1-4 m_{h}^{2} / s}\left[\left(2 m_{h}^{2}+s\right)^{2}+m_{h}^{2} \Gamma_{h}^{2}\right]}{\sqrt{1-4 m_{\phi_{1}}^{2} / s}\left[\left(m_{h}^{2}-s\right)^{2}+m_{h}^{2} \Gamma_{h}^{2}\right]} .
$$

The thermally averaged cross sections and relic abundances are computed following the prescription described in Appendix B.II. The results of the scan are given in Fig. 3.3 (bottom panel). In Fig. 3.7 we plot the coupling $\lambda_{H}$ necessary to obtain correct DM relic density as a function of the DM mass, $m_{\phi_{1}}$. As commented in Sec. 3.3.2 the flavon vevs split the lightest DM state $\phi_{1}$ from the heavier ones, such that only $\phi_{1}$ contributes to $\Omega_{\mathrm{DM}}$ (lower dashed line). Instead, if the splitting is too small for $\phi_{2,3}$ to decay before freeze-out, all three components contribute (upper dashed line). In both cases, requiring the Higgs-portal coupling $\lambda_{H}<\sqrt{4 \pi}$, such that the relic-abundance calculation is well in the perturbative regime, limits the DM mass $m_{\phi_{1}} \lesssim 8 \mathrm{TeV}$.

Note that the role of the Higgs portal may be played by other light scalars. In Ref. [97] the flavon field of the Abelian horizontal symmetry was used to enhance the DM annihilation cross section. If the flavons are light, they can also modify the phenomenology of the fermionic flavored DM, allowing DM annihilation into flavons. In this case the DM phenomenology of the fermionic flavored DM would be closer to the one of our scalar DM model. 


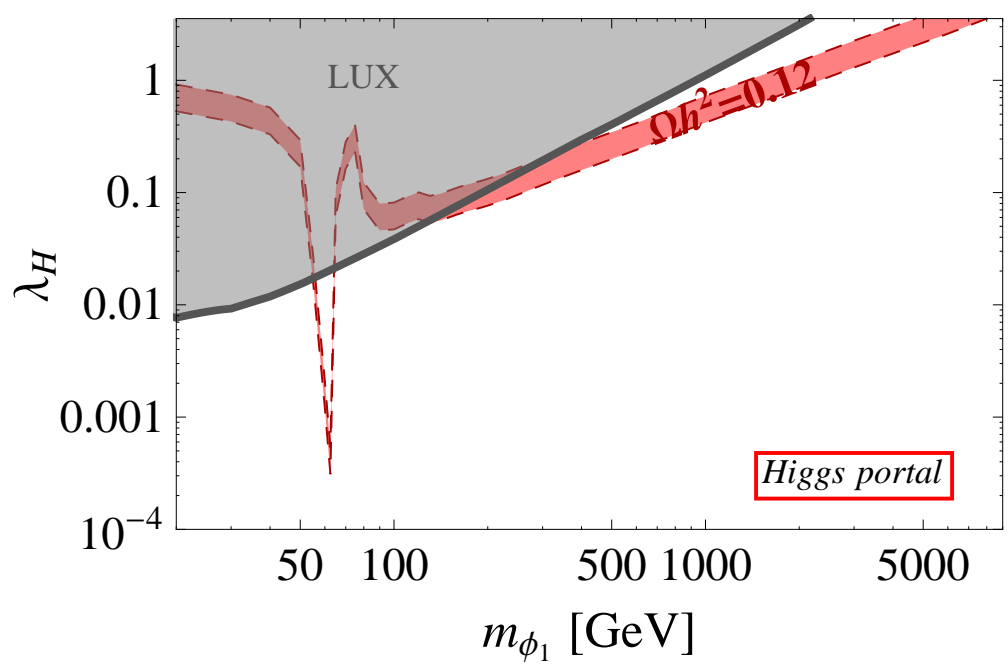

Figure 3.7: The Higgs-DM coupling, $\lambda_{H}$, as a function of DM mass that gives the correct relic abundance for the Higgs portal scalar DM (red band). The upper (lower) dashed edge corresponds to the limit where $\phi_{2,3}$ decay much after (before) the thermal freeze-out of $\phi_{1}$. The LUX bound, assuming correct relic abundance, is shown as a shaded grey region.

\subsubsection{Cosmology}

The heavier flavored DM states, both for the fermionic DM, $\chi_{2,3}$, and scalar DM, $\phi_{2,3}$, are unstable. They decay through the $\chi_{i} \rightarrow \chi_{j} \bar{q} q^{\prime}$ transition when the mass splitting is larger than the pion mass, and through the $\chi_{i} \rightarrow \chi_{j} \gamma \gamma$ otherwise, cf. Eqs. (3.17), (3.18). The SM particles in the final state of these decays can have various observable effects in cosmology and astrophysics.

The two relevant sets of parameters are the lifetimes of the two heavy states, $\tau_{\chi_{2,3}}$, and the related mass splittings of the DM multiplet (with respect to the lightest state), $\Delta m_{31}, \Delta m_{21}$. The lifetimes determine at which cosmological epoch the heavy states decay. The mass splittings control the released combined electromagnetic and hadronic energy, $E_{\mathrm{vis}} \simeq \Delta m_{21,31}$. They also determine the relic abundances of the heavy states. Generically, near the degenerate limit each state contributes roughly a third of the total 
DM relic abundance, $\Omega_{\mathrm{DM}}$. Close to the resonant condition $m_{\chi} \simeq m_{A^{24}} / 2$ the $\chi_{1,2,3}$ relic abundances may differ from $\Omega_{\mathrm{DM}} / 3$, depending on the common DM mass and relative mass splittings.

For the scalar DM the mass splitting is expected to be large. The $\phi_{2,3}$ therefore decay before primordial nucleosynthesis. The decays yield negligible entropy release due to the small $\phi_{2,3}$ abundances. Such scenarios are basically unconstrained by current cosmological observations. The same is true for the fermionic DM if additional spurions split the DM multiplet at tree level.

If the fermionic DM multiplet is split solely by radiative corrections, the $\chi_{2,3}$ and $\chi_{1}$ are generically much more degenerate, cf. Fig. 3.4 (right). The $\chi_{2,3}$ states are then potentially long lived. For $\tau_{2,3} \sim \mathcal{O}\left(10^{-1} \mathrm{~s}-10^{12} \mathrm{~s}\right)$ the decays may affect the primordial generation of light nuclear elements [107]. For longer lifetimes, $\tau_{2,3} \sim \mathcal{O}\left(10^{10} \mathrm{~s}-10^{13} \mathrm{~s}\right)$, the $\chi_{2,3}$ decays distort the thermalization of the CMB by injecting high-energy photons into the plasma before recombination, which is strongly constrained $[108,109]$. The $\chi_{2,3}$ states with lifetimes longer than $\tau_{2,3} \gtrsim 10^{10} \mathrm{~s}$ are ruled out, if the injected photons carry energy above the thresholds of the efficient thermalization processes. Typically this is a fraction of the electron mass. For even longer lifetimes, $\tau_{2,3} \gtrsim 10^{13} \mathrm{~s}$, the $\chi_{2,3}$ states decay after recombination. This results in photons that free-stream to us and can be searched for in diffuse galactic and extra-galactic gamma and X-ray spectra. A combination of measurements excludes scenarios with $\tau_{2,3} \lesssim 10^{26} \mathrm{~s}$ all the way down to $\Delta m_{21,31} \gtrsim \mathcal{O}(10) \mathrm{keV}[110]$.

In the remainder of this section we consider in more detail the region $\tau_{\chi 2,3} \sim 10^{-1} \mathrm{~s}-$ $10^{12} \mathrm{~s}$, where the big bang nucleosynthesis $(\mathrm{BBN})$ provides the most stringent constraints $[107,111]$. The injection of energetic photons or hadrons from $\chi_{2,3}$ decays during or after BBN adds an additional non-thermal component to the plasma that can modify the abundances of the light elements [112-116]. The bounds differ depending on whether the decays result in hadronic or electromagnetic showers in the plasma. 
The most stringent bounds are for a relic that produces mostly hadronic showers. This is because the electromagnetically interacting particles such as photons and electrons thermalize very quickly by interacting with the tail of the $\mathrm{CMB}$ distribution until the universe is $10^{6} \mathrm{~s}$ old. In our case, the decays $\chi_{2,3} \rightarrow \chi_{1} q \bar{q}^{\prime}$ are always kinematically allowed for $\tau_{\chi_{2,3}}<10^{12} \mathrm{~s}$. The $\chi_{2,3}$ decays thus predominantly produce a small number of hadronic jets with a combined released hadronic energy $E_{\mathrm{had}} \simeq E_{\mathrm{vis}}$.

There are three distinct ranges of lifetimes [117]. For $0.1 \mathrm{~s} \lesssim \tau_{\chi_{2,3}} \lesssim 100 \mathrm{~s}$ the dominant effect is the inter-conversion between protons and neutrons, which overproduces the ${ }^{4} \mathrm{He}$ abundance. For longer lifetimes, $100 \mathrm{~s} \lesssim \tau_{\chi_{2,3}} \lesssim 10^{7} \mathrm{~s}$, hadro-dissociation is the most efficient process and the bounds come from the non-thermal production of $\mathrm{Li}$ and D. At late times, $10^{7} \mathrm{~s} \lesssim \tau_{\chi 2,3} \lesssim 10^{12} \mathrm{~s}$, photo-dissociation caused by direct electromagnetic showers or by electromagnetic showers from daughter hadrons can lead to overproduction of ${ }^{3} \mathrm{He}$.

We impose the ${ }^{4} \mathrm{He}, \mathrm{D}$ and ${ }^{3} \mathrm{He}$ constraints ${ }^{1}$ using the results in Ref. [117]. The visible energy release in the decays is $E_{\mathrm{vis}} \sim \Delta m_{21,31}$. For $100 \mathrm{GeV}<\Delta m_{21,31}<10 \mathrm{TeV}$ the constraints derived from the three relic mass benchmarks in Ref. [117] are well approximated by a power-law scaling with $E_{\mathrm{vis}}^{-\eta_{i}}$. The exponents for the three constraints are $\eta_{4} \mathrm{He} \approx 1 / 3, \eta_{\mathrm{D}} \approx 1 / 2$ and $\eta_{3} \mathrm{He} \approx 1$. For inter-conversion and hadro-dissociation the power-law scaling is expected to break down at energies below $\mathcal{O}(10) \mathrm{GeV}$ due to the presence of hadronic thresholds [117]. We thus do not extrapolate the fit results for ${ }^{4} \mathrm{He}$ and $\mathrm{D}$ for $\Delta m_{21,31}$ below $10 \mathrm{GeV}$. We assume that the photo-dissociation effects retain approximate power law behavior for $E_{\mathrm{vis}}$ large compared to the binding energies of the light nuclei, which is of the order of few tens of MeV. In our model for $\tau_{\chi 2,3}<10^{2(12)} \mathrm{s}$, the mass splitting, $\Delta m_{21,31}$, is always above $10(0.1) \mathrm{GeV}$. Our approximations are thus

\footnotetext{
${ }^{1}$ The measured ${ }^{4} \mathrm{He}$ abundance has shifted upwards significantly since the publicaton of Ref. [117]. This should weaken the constraints for $\tau_{2,3} \lesssim 100 \mathrm{~s}$. The upward shift has no consequences for our conclusions since we find that the ${ }^{4} \mathrm{He}$ constraint from Ref. [117] is already never important in excluding the viable parameter space in our models.
} 
always valid for ranges of lifetimes for which the ${ }^{3} \mathrm{He}$ constraints are the most strin-

gent. For the deuterium bound, on the other hand, the power-law scaling is expected to fail for part of the parameter space where the bound is the most stringent, since $\Delta m_{21,31}$ can be as low as a few $\mathrm{GeV}$. We have checked using the power-law derived bound that these regions are excluded by several orders of magnitude. This gives us confidence to conclude that they remain excluded even with a more faithful treatment of hadro-dissociation effects.

In Fig. 3.5 we show the distribution of $\chi_{2,3}$ lifetimes in the viable parameter space of the fermionic DM model. The cosmological constraints rule out all points with $\tau_{2,3} \gtrsim 100 \mathrm{~s}$, which is the range of lifetimes for which the deuterium bound becomes effective. The points with lifetimes $\tau_{2,3} \lesssim 100 \mathrm{~s}$, on the other hand, are never excluded by cosmological constraints. This is the range of lifetimes where the most strigent bound comes from the ${ }^{4} \mathrm{He}$ abundance, which, however, is not sufficient to exclude any of our fermionic DM model points.

\subsubsection{Direct and indirect dark matter searches}

Both fermionic and scalar flavored DM can produce direct detection signal from DM scattering on nuclei. For fermionic DM the scattering is due to $t$-channel exchanges of FGBs. For scalar DM the scattering is dominated by the Higgs exchange in the $t$ channel, while the contribution of FGBs is in general negligible.

The spin-independent interactions with the nucleons for the fermionic flavored DM are described by the following effective Lagrangian $[118,119]$

$$
\mathcal{L}_{\text {dir. }}=f_{p}\left(\bar{\chi} \gamma_{\mu} \chi\right)\left(\bar{p} \gamma^{\mu} p\right)+f_{n}\left(\bar{\chi} \gamma_{\mu} \chi\right)\left(\bar{n} \gamma^{\mu} n\right)
$$

The Wilson coefficients $f_{p}$ and $f_{n}$ are the couplings to protons and neutrons, respec- 
tively,

$$
f_{p}=\sum_{m}\left(\hat{g}_{\chi}^{m}\right)_{11} \frac{2\left(\hat{\mathcal{G}}_{V}^{u}\right)_{11, m}+\left(\hat{\mathcal{G}}_{V}^{d}\right)_{11, m}}{m_{A^{m}}^{2}} \quad \text { and } \quad f_{n}=\sum_{m}\left(\hat{g}_{\chi}^{m}\right)_{11} \frac{\left(\hat{\mathcal{G}}_{V}^{u}\right)_{11, m}+2\left(\hat{\mathcal{G}}_{V}^{d}\right)_{11, m}}{m_{A^{m}}^{2}} .
$$

$\hat{\mathcal{G}}_{V}^{u, d}$ are the vectorial couplings of FGBs to quarks, defined in Eq. (3.25). The spinindependent DM-nucleon cross section as measured by the LUX experiment [120] is

$$
\sigma_{\chi N}^{\mathrm{SI}}=\left[1+\frac{Z}{A}\left(\frac{f_{p}}{f_{n}}-1\right)\right]^{2} \frac{\mu_{\chi n}^{2} f_{n}^{2}}{\pi}
$$

where $\mu_{\chi n}$ is the reduced mass of the $(\chi, n)$ system. The Xenon atomic and mass numbers are denoted by $Z$ and $A$, respectively. We thus have $Z=54$, while $A$ varies between 128 and 134. With the above relations we calculate the DM-nucleon cross section and compare it with the current best limits reported by the LUX experiment [120]. The results of the scan are shown in Fig. 3.8. Most of the points lie well below the present LUX bound. This is a consequence of the fact that the relic abundance is given by the $s$-channel resonant annihilation, while the direct detection scattering is due to $t$-channel exchanges of FGBs and thus not resonantly enhanced.

For scalar flavored DM the dominant scattering is through $t$-channel Higgs-boson exchange. This leads to the spin-independent scattering on nucleon $N=n, p,[121,122]$

$$
\sigma_{\chi N}^{\mathrm{SI}}=\frac{\lambda_{H}^{2} f_{N, h}^{2}}{4 \pi}\left(\frac{m_{\phi_{1}} m_{N}}{m_{\phi_{1}}+m_{N}}\right)^{2} \frac{m_{N}^{2}}{m_{h}^{4} m_{\phi_{1}}^{2}} .
$$

The Higgs-nucleon coupling is

$$
f_{N, h}=\frac{2}{9}+\frac{7}{9} \sum_{q} f_{q}^{(N)}
$$

where the sum runs over the light quarks, $q=u, d, s . f_{q}^{(N)}$ are defined by the matrix 


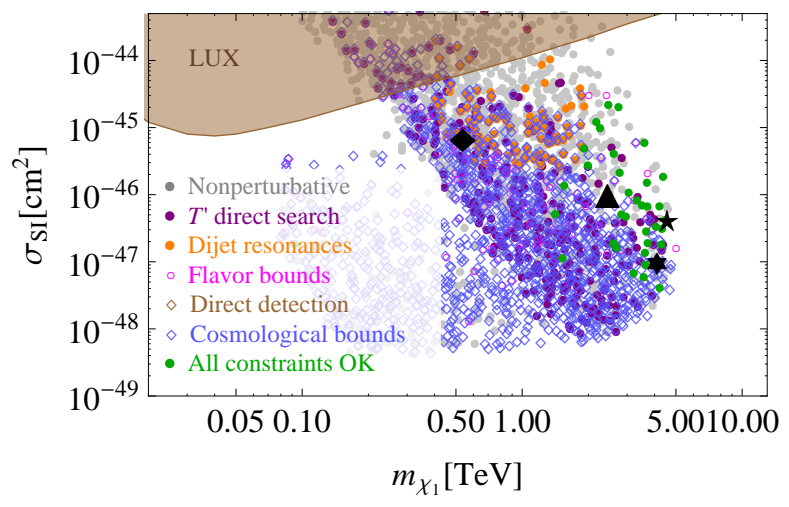

Figure 3.8: The predicted spin-independent cross section for DM scattering on nuclei as a function of DM mass for radiatively split fermionic DM (left) and in the large masssplitting limit (right). The LUX bound is the brown shaded region. The color coding for the points is as in Fig. 3.3.

elements of the light-quark scalar currents, $m_{N} f_{q}^{(N)} \equiv\left\langle N\left|m_{q} \bar{q} q\right| N\right\rangle$. For the $s$ quark we use the lattice determination $f_{s}^{(N)}=0.043 \pm 0.011$ [123]. The matrix elements for $u$ and $d$ quarks depend strongly on $\pi N$-scattering data. A Baryon Chiral Perturbation Theory (BoPA) analysis of the $\pi N$-scattering data gives $\sigma_{\pi N}=59(7) \mathrm{MeV}$ [124]. This

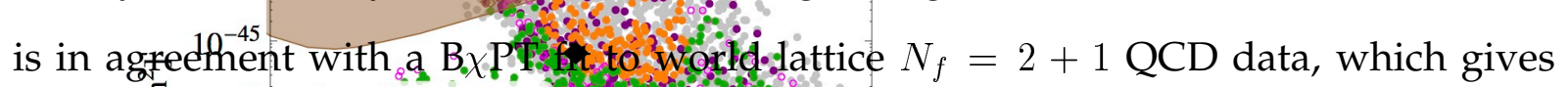

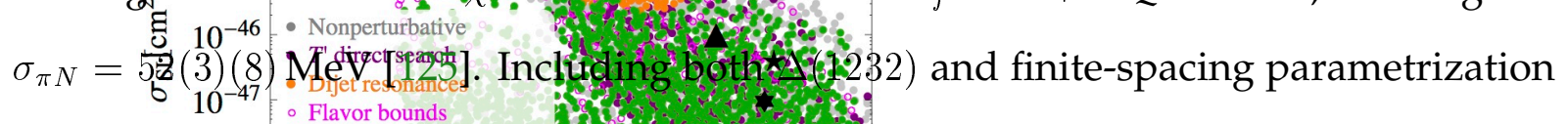
in the fit shifts thiecterntral value to $\sigma=4 . \mathrm{MeV}$. To be conservative we use $\sigma_{\pi N}=$

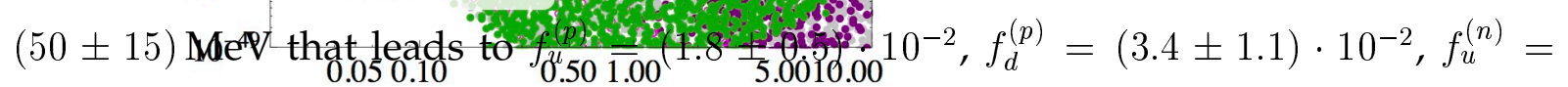
$\left.(1.6 \pm 0.5) \cdot 10^{-2}, f_{d}^{(n)}=\left(3.8 n+\frac{w_{+}}{\text {T TeY }}\right)\right] \cdot 10^{-2}$ by using expressions in Refs. [126, 127]. This results in $f_{N, h}=(29.7 \pm 1.3) \cdot 10^{-2}$ where we averaged over Higgs couplings to proton and neutron (the difference is an order of magnitude smaller than the quoted error). The resulting bound from the LUX experiment, assuming correct relic abundance, is shown in Fig. 3.7 and constrains $m_{\phi_{1}} \gtrsim 150 \mathrm{GeV}$.

Finally, we discuss the constraints from indirect DM searches. The flavored DM annihilates to quarks so that the most constraining indirect DM searches are due to the photon and antiproton cosmic-ray fluxes. The constraints from the antiproton flux 
Figure 3.9: The dijet production cross section at $8 \mathrm{TeV}$ LHC as a function of the lightest FGB mass for radiatively split fermionic DM (left) and in the large mass splitting limit (right). The 95\% CL limit from Ref. [133] is denoted with a solid orange line. The color coding is the same as in Fig. 3.4.

are quite dependent on the propagation model. This can lead to almost an order of magnitude difference in uncertainty on the value of the excluded annihilation cross section [128].

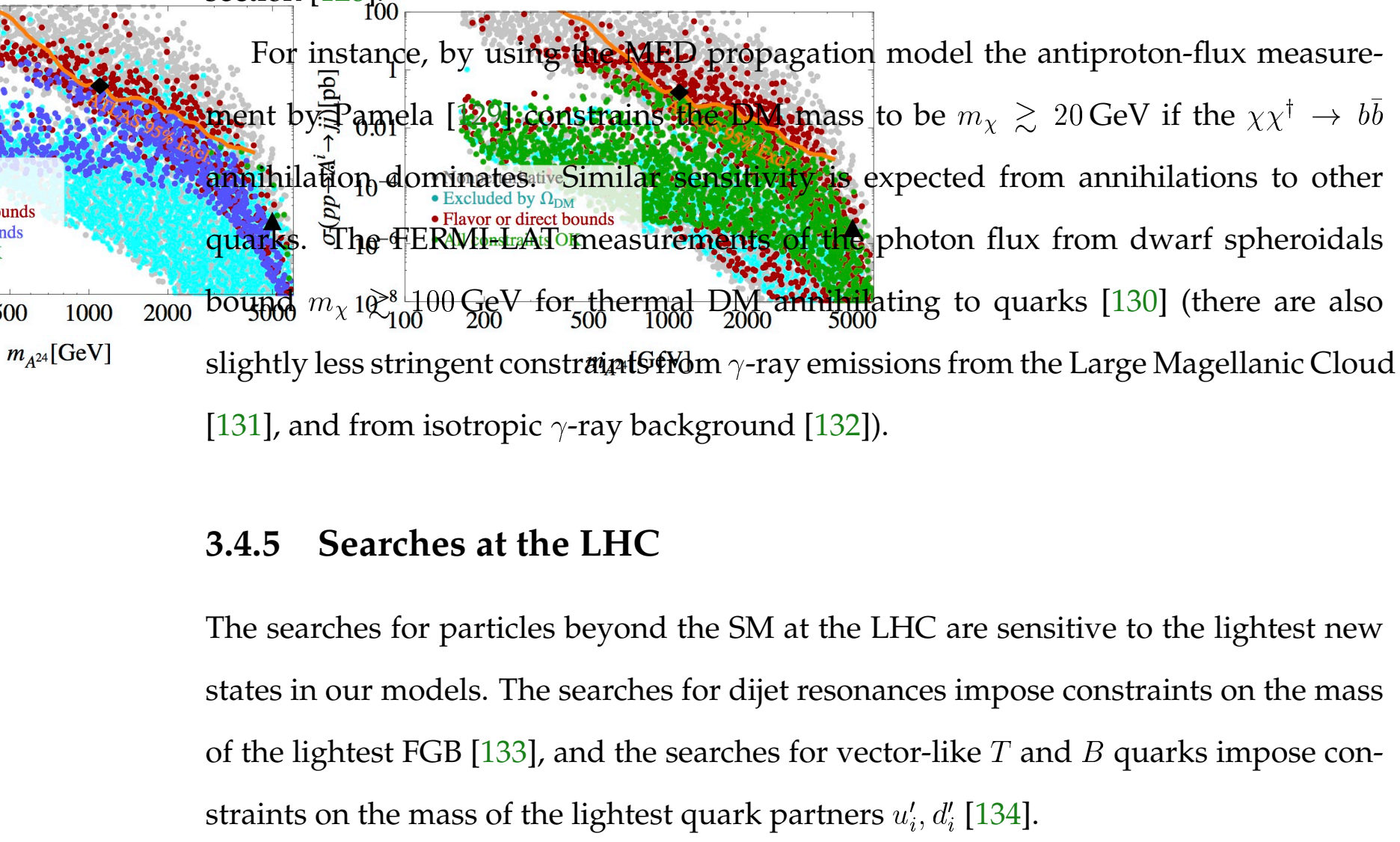

\subsubsection{Searches at the LHC}

The searches for particles beyond the SM at the LHC are sensitive to the lightest new states in our models. The searches for dijet resonances impose constraints on the mass of the lightest FGB [133], and the searches for vector-like $T$ and $B$ quarks impose constraints on the mass of the lightest quark partners $u_{i}^{\prime}, d_{i}^{\prime}$ [134]. 
The FGBs are narrow resonances that have flavor-conserving as well as flavorviolating couplings to the SM quarks, $u_{i}, d_{i}$, and to the quark partners, $u_{i}^{\prime}, d_{i}^{\prime}$. Since the FGBs are not colored they do not directly couple to gluons. At the partonic level the production process is dominated by $q_{i} \bar{q}_{j} \rightarrow A^{m} \rightarrow q_{k} \bar{q}_{l}$. The FGBs would then appear as resonances in the dijet invariant-mass spectrum. For the most part, the LHC dijet resonance searches are relevant only for the lightest FGB which has, to a very good approximation, flavor-diagonal couplings to quarks. In this case, the cross section for $p p \rightarrow j j$ is given by $[135,136]$

$$
\sigma(p p \rightarrow q \bar{q})=\sum_{i, j} \int_{4 m_{j}^{2}}^{s} \frac{d \mathscr{M}^{2}}{s} \int_{-Y_{B}}^{Y_{B}} d y_{B} \int_{-z_{o}}^{z_{o}} d z f_{i}\left(\sqrt{\tau} e^{y_{B}}\right) f_{\bar{i}}\left(\sqrt{\tau} e^{-y_{B}}\right) \frac{1}{2} \frac{d}{d z} \hat{\sigma}_{i j} .
$$

The partonic differential cross section is given by

$$
\begin{aligned}
\frac{d}{d z} \hat{\sigma}_{i j} & =\frac{1}{32 \pi} \beta_{f} \frac{\mathscr{M}^{2}}{\left(\mathscr{M}^{2}-m_{A^{m}}^{2}\right)^{2}+m_{A^{m}}^{2} \Gamma_{A^{m}}^{2}}\left(\left|\hat{\mathcal{G}}_{V}^{u, d}\right|_{i i, m}^{2}+\left|\hat{\mathcal{G}}_{A}^{u, d}\right|_{i i, m}^{2}\right) \\
& \times\left[\left(\left|\hat{\mathcal{G}}_{V}^{u, d}\right|_{j j, m}^{2}+\left|\hat{\mathcal{G}}_{A}^{u, d}\right|_{j j, m}^{2}\right)\left(1+\beta_{f}^{2} z^{2}\right)+4\left(\left|\hat{\mathcal{G}}_{V}^{u, d}\right|_{j j, m}^{2}-\left|\hat{\mathcal{G}}_{A}^{u, d}\right|_{j j, m}^{2}\right) \frac{m_{j}^{2}}{\mathscr{M}^{2}}\right],
\end{aligned}
$$

where, in the partonic center of mass frame, $\mathscr{M}$ is the total energy, $\beta_{f}$ is the velocity of the final-state quarks, $z=\cos \theta^{*}$ is the cosine of the polar angle of the outgoing quark w.r.t. the direction of the incoming quark, and the couplings $\hat{\mathcal{G}}_{V}, \hat{\mathcal{G}}_{A}$ of FGBs to quarks were defined in Eq. (3.25). We have only included the $s$-channel contribution that dominates on the FGB resonance peak. Terms odd in $z$ were dropped in the differential cross section since they vanish after integration. The predicted dijet cross sections at the LHC with $\sqrt{s}=8 \mathrm{TeV}$ are shown in Fig. 3.9, where the 95\% CL exclusion from Ref. [133] is denoted with a solid orange line. This mostly excludes the points where the lightest FGB has large couplings to the quarks. Such points are in fact already mostly excluded either by the perturbativity requirement or from flavor constraints. 
The quark partners, $u_{i}^{\prime}, d_{i}^{\prime}$, have an inverted mass hierarchy w.r.t. the SM quarks so that in most of our scan points the $t^{\prime}$ is the lightest state. The bound on the $t^{\prime}$ mass depends on the $t^{\prime} \rightarrow b W, t Z$, and $t h$ branching ratios. The respective partial decay widths are given by

$$
\begin{aligned}
& \Gamma\left(t^{\prime} \rightarrow b W\right)= \frac{g_{w}^{2}}{64 \pi}\left|s_{u_{L} 3} V_{33} c_{d_{L} 3}\right|^{2} \frac{m_{t^{\prime}}^{3}}{m_{W}^{2}}\left(1-x_{W}^{2}\right)^{2}\left(1+2 x_{W}^{2}\right) \\
& \Gamma\left(t^{\prime} \rightarrow t Z\right)=\frac{g_{w}^{2}}{128 \pi}\left(c_{u_{L} 3} s_{u_{L} 3}\right)^{2} \frac{m_{t^{\prime}}^{3}}{m_{W}^{2}} \sqrt{\left[1-\left(x_{Z}+x_{t}\right)^{2}\right]\left[1-\left(x_{Z}-x_{t}\right)^{2}\right]} \\
& \times\left[\left(1-x_{Z}^{2}\right)\left(1+2 x_{Z}^{2}-x_{t}^{2}\right)-x_{t}^{2}\left(1-x_{t}^{2}\right)\right] \\
& \Gamma\left(t^{\prime} \rightarrow t h\right)=\frac{\lambda_{u}^{2}}{64 \pi} m_{t^{\prime}} \sqrt{\left[1-\left(x_{h}+x_{t}\right)^{2}\right]\left[1-\left(x_{h}-x_{t}\right)^{2}\right]} \\
& \quad \times\left[\left(s_{u_{R} 3}^{2} s_{u_{L} 3}^{2}+c_{u_{R} 3}^{2} c_{u_{L} 3}^{2}\right)\left(1+x_{t}^{2}-x_{h}^{2}\right)-4 s_{u_{R} 3} s_{u_{L} 3} c_{u_{R} 3} c_{u_{L} 3} x_{t}\right]
\end{aligned}
$$

where $x_{i}=m_{i} / m_{t^{\prime}}$ and $s_{i}, c_{i}$ are the sines and cosines of the mixing angles between the SM and exotic quarks, while $V$ is a unitary matrix describing the misalignment of the $Y_{u}$ and $Y_{d}$ vevs. Their definitions can be found in Ref. [102], where also the relevant Feynman rules are given. (We present the relevant Higgs Feynman rules in App. B.III, correcting an obvious typographical error of Ref. [102].) In Eq. (3.38) we took the limit $x_{b} \rightarrow 0$ that is justified since $m_{t^{\prime}} \gg m_{b}$. We use the above expressions for the $t^{\prime} \rightarrow b W, t Z, t h$ branching ratios to obtain the $95 \%$ confidence-level bound on the $t^{\prime}$ mass by interpolating between quoted observed-limits table in [134].

\subsubsection{Flavor constraints}

The model of gauged-flavor symmetries in Eq. (3.6) was designed to be compatible with new $\mathrm{TeV}$-scale dynamics and at the same time satisfy the tight flavor constraints from FCNC observables. The FCNCs induced by the exchange of new states are thus 
relatively mild. The light flavor-violating gauge bosons mediate $\Delta \mathrm{F}=2$ transitions at the tree-level, while the light exotic quarks modify the loop-induced SM process. These modifications are large enough that they restrict the parameter space of the model [102]. All the flavor-violating parameters in the model are fixed by requiring $\left\langle Y_{u}\right\rangle$ and $\left\langle Y_{d}\right\rangle$ to reproduce the observed masses and mixings in the quark sector. The size of the induced FCNCs thus depend only on a relatively small set of flavor conserving parameters in the model, the flavor symmetric masses and couplings. Following the analysis in Ref. [102] we focus on $\Delta \mathrm{F}=2$ observables in the neutral $B$ and $K$ sectors, and on $\bar{B}_{s} \rightarrow X_{s} \gamma$.

In our analysis we include the mass differences in the neutral $K^{0}, B_{s}^{0}$, and $B_{d}^{0}$ sectors, $\Delta m_{K}, \Delta m_{B_{d}}$, and $\Delta m_{B_{s}}$, respectively. We also include the indirect CP violation in the kaon sector, $\varepsilon_{K}$, and the mixing-induced CP asymmetries $S_{\psi K_{s}}$ and $S_{\psi \phi}$. The corresponding mixing amplitude

$$
2 m_{M}\left(M_{12}^{M}\right)^{*}=\left\langle\bar{M}\left|\mathcal{H}_{\mathrm{eff}}^{\Delta M=2}\right| M\right\rangle,
$$

where $M=K^{0}, B_{d}^{0}, B_{s}^{0}$, controls all of these observables.

Two NP contributions to $M_{12}^{M}$ dominate. These are the tree-level exchanges of FGBs and the loop-induced SM-like contribution with internal up-type quarks, including exotic quarks. For the later contribution we first integrate out at the EW scale, $\mu_{W} \simeq m_{W}$, the exotic quarks together with the $W$ and the top quark. In this step we ignore the hierarchy of masses between the exotic quarks and top. This is a good approximation for the dominant contribution that comes from $t^{\prime}$. The theory matches onto the EFT with the SM effective weak operators. We perform the Renormalization Group (RG) of the Wilson coefficients to the low scale at which the hadronic matrix elements are evaluated on the lattice. For the tree-level FGB exchanges the hard scale is given by the corresponding gauge-boson masses. We integrate out the FGB at the correspond- 
ing hard scale and RG evolve the Wilson coefficients to the hadronic scale. The FGB exchanges generate four-fermion operators with the Dirac structures that differ from the SM one, namely $\left(\bar{f}_{i} \gamma_{\mu} P_{R} f_{j}\right)\left(\bar{f}_{j} \gamma^{\mu} P_{R} f_{i}\right)$ and $\left(\bar{f}_{i} \gamma_{\mu} P_{R} f_{j}\right)\left(\bar{f}_{j} \gamma^{\mu} P_{L} f_{i}\right)$, where $i, j$ are the flavor indices. The RG evolution is implemented following Ref. [137] (for further details and the dependence of the numerical relevance with the scale see also Ref. [102]). For the non-perturbative inputs, the decay constants and the bag parameters, we use the current lattice averages [138].

The mass difference in the neutral kaon sector, $\Delta m_{K}$, and the $\mathrm{CP}$-violating parameter $\varepsilon_{K}$ are given by

$$
\Delta M_{K}=2 \operatorname{Re}\left(M_{12}^{K^{0}}\right), \quad \varepsilon_{K}=\frac{\kappa_{\varepsilon} e^{i \varphi_{\varepsilon}}}{\sqrt{2} \Delta M_{K}^{\exp }} \operatorname{Im}\left(M_{12}^{K^{0}}\right),
$$

with $\varphi_{\varepsilon}=(43.51 \pm 0.05)^{\circ}$ and $\kappa_{\varepsilon}=0.923 \pm 0.006$, which includes long-distance effects in both $\operatorname{Im} M_{12}^{K^{0}}$ [139] and in the decay, i.e. $\operatorname{Im} \Gamma_{12}^{K^{0}}$ [140]. Our SM expectation for $\varepsilon_{K}$ incorporates the known Next-to-Next-to-Leading-Order (NNLO) QCD corrections due to the charm [141] and charm-top [142] contributions.

The mass differences in the neutral $B$ sectors are given by

$$
\Delta M_{B_{q}}=2\left|M_{12}^{B_{q}}\right|, \quad \text { with } q=d, s .
$$

The $\mathrm{CP}$ violation in the neutral $B$ sector is probed by the time-dependent asymmetries in the decays $B_{d}^{0} \rightarrow \psi K_{S}$ and $B_{s}^{0} \rightarrow \psi \phi$ that define the observables

$$
S_{\psi K_{s}}=\sin \left(2 \beta+2 \phi_{B_{d}}\right) \quad \text { and } \quad S_{\psi \phi}=\sin \left(2\left|\beta_{s}\right|+2 \phi_{B_{s}}\right),
$$

respectively. In the conventional parametrization of the CKM matrix the SM phases are given by $V_{t d}^{\mathrm{SM}}=\left|V_{t d}^{\mathrm{SM}}\right| e^{-i \beta}$ and $V_{t s}^{\mathrm{SM}}=-\left|V_{t s}^{\mathrm{SM}}\right| e^{-i \beta_{s}}$. The NP phases are defined through the relation $M_{12}^{B_{q}}=\left|M_{12}^{B_{q}}\right| e^{2 i\left(\beta_{q}+\phi_{B_{q}}\right)}$. The tree level exchanges of FGBs induce 
such new phases in $\Delta F=2$ matrix elements. These are thus constrained both by the $S_{\psi K_{S}}$ and $S_{\psi \phi}$ asymmetries, and by $\varepsilon_{K}$ in the kaon sector.

The rate of $\bar{B} \rightarrow X_{s} \gamma$ is also modified by the presence of exotic up-type quarks. These can only enhance the $\bar{B} \rightarrow X_{s} \gamma$ rate with respect to the SM expectations [91]. The contributions of FGBs are loop-suppressed. Even though they may be enhanced by $m_{b^{\prime}}$ they are negligible in models with a seesaw-like mass generation for quarks, like the model we consider [143]. The SM prediction for the rate in our analysis includes the known NNLO corrections [144-146].

In our numerical scan we mark parameter space points to have passed the flavor constraints only if the predictions for all our observables lie within three standard deviations of the corresponding experimental values. Whenever theoretical uncertainties are relevant, we include them in quadrature with the experimental ones.

The deviations of the selected FCNC observables from the SM predictions for the four benchmark points are shown in Figs. 3.10 to 3.12 .

\subsection{Benchmarks}

To illustrate the most relevant phenomenology of fermionic flavored DM we select four representative benchmark points. The main features of the four benchmarks are summarized in Figs. 3.10-3.13. The upper panels in the figures show the spectra for the FGBs, $A^{m}$, the quark partners, $u_{i}^{\prime}, d_{i}^{\prime}$, and the DM multiplet, $\chi_{i}$. Each FGB is represented by four shaded $3 \times 3$ rasters. The shade of the entries in the rasters is approximately logarithmically proportional to the size of the couplings to $u_{R}, d_{R}, u_{L}$ and $d_{L}$, respectively (from left to right). The DM relic abundances as functions of the $\chi_{1}$ mass are shown in the bottom left panels. The lines correspond to our approximate calculation

for a radiatively split DM multiplet (red solid line) and for a DM multiplet with large mass splittings (black dashed line). The open diamonds (circles) correspond to the 
solutions of the coupled Boltzmann equations for the radiative (large) splitting cases which were calculated in MadDM. The approximate and MadDM relic-abundance calculations are in very good agreement for this small subset of benchmarks. In general, however, a disagreement of up $\mathcal{O}(30 \%)$ could be expected due to the approximations (see App. B.II for a more detailed discussion). The bottom right panels show the pull in selected flavor observables, i.e., the differences between theoretical predictions and measurements normalized to the 1- $\sigma$ uncertainties. The uncertainties were obtained by adding in quadrature the theory and experimental errors. The four benchmark points are also marked in Figs. 3.3, 3.4, and 3.5 with a diamond (1), a five-point star (2), a triangle (3), and a six-point star (4).

"Benchmark 1" is an example of fermionic flavored DM, where the DM multiplet is light, with mass below $1 \mathrm{TeV}$. The mass of the lightest state in the DM multiplet is $m_{\chi_{1}} \simeq$ $520(540) \mathrm{GeV}$ if it lies just below (above) the LFGB resonance. If the mass splitting between $\chi_{1}, \chi_{2}$ and $\chi_{3}$ is solely due to radiative corrections, $\chi_{2}$ and $\chi_{3}$ are almost mass degenerate with masses roughly $10 \mathrm{GeV}$ above $m_{\chi_{1}}$, and $\chi_{3}$ is about $100 \mathrm{MeV}$ heavier than $\chi_{2}$. The lightest quark partner is $t^{\prime}$ with a mass $m_{t^{\prime}} \simeq 1.3 \mathrm{TeV}$. The lightest FGB has a mass $m_{A^{24}} \simeq 1.1 \mathrm{TeV}$. All the remaining NP states are above $7 \mathrm{TeV}$. This benchmark point demonstrates that even parameter regions with low lying FGBs can be consistent with both the resonance searches and the FCNC bounds. The most robust constraints in this parameter region come from cosmology (in case of radiatively-split DM masses) and dijet-resonance searches (see Figs. 3.5 and 3.9). Note in particular that for the completely (mass) decoupled fermionic DM scenario, in which cosmology bounds are absent, all experimental constraints can be satisfied even for DM (and LFGB) masses below few $100 \mathrm{GeV}$.

The bottom left panel in Fig. 3.10 shows the predicted relic abundance for this benchmark, if only the DM mass is varied, which also modifies the splitting within the DM triplet. Relic abundance consistent with observations is obtained for a mass of 


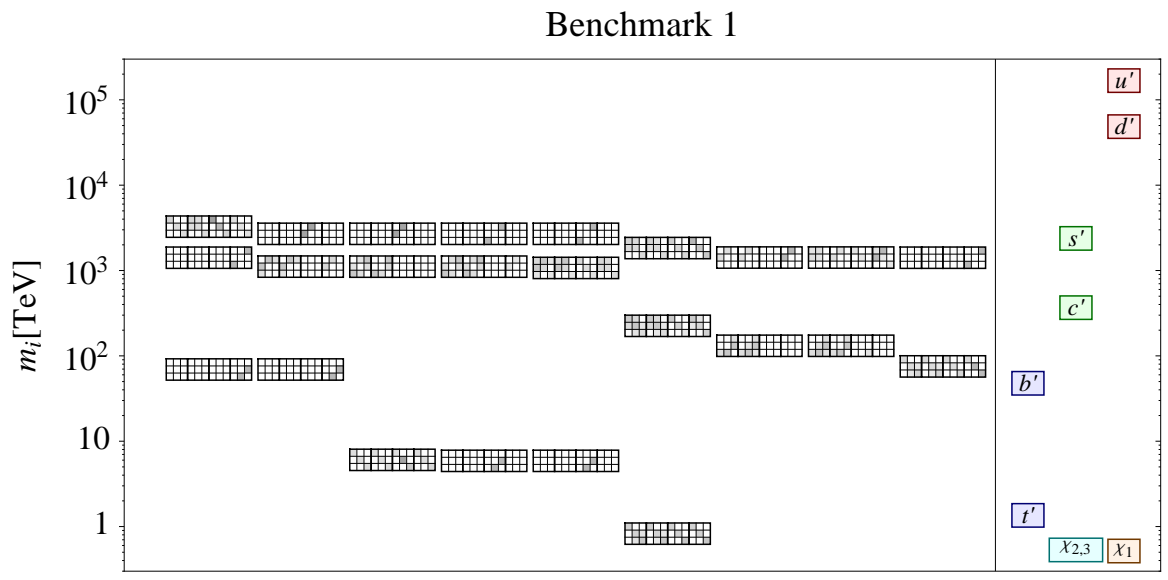

$M_{u}=980 . \mathrm{GeV}, \lambda_{u}=1 ., \lambda_{u}^{\prime}=11.8, M_{d}=700 . \mathrm{GeV}, \lambda_{d}=0.82, \lambda_{d}^{\prime}=3.8, g_{Q}=0.126, g_{U}=0.41, g_{D}=0.237$
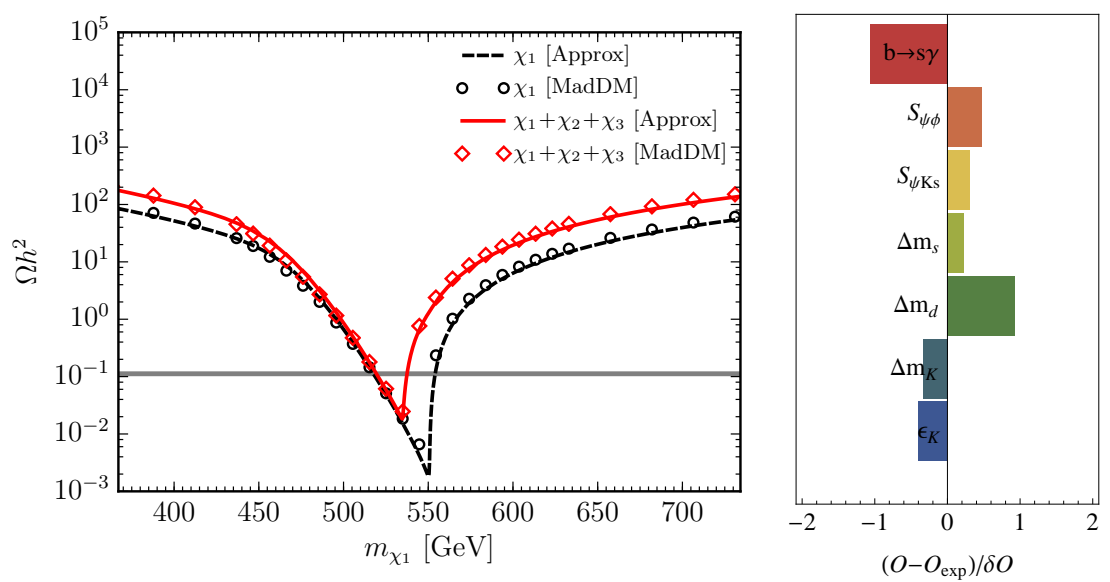

Figure 3.10: Mass spectrum and flavor decomposition (upper panel), DM relic density as a function of the DM mass with all other parameters fixed (lower left panel) and the pattern of effects in selected flavor observables (lower right panel) for the fermionic flavored DM benchmark 1. The input benchmark-point parameters are listed in the center. See text for details. 
DM close to half of the mass of the lightest FGB, in which case the annihilation cross section is resonantly enhanced. To saturate the observed DM relic density, two solutions for $m_{\chi_{1}}$ are obtained, with $m_{\chi_{1}}$ either above or below the resonant peak. We see that for radiatively split DM masses, where all $\chi_{i}$ components contribute to the DM relic density, $m_{\chi_{i}}$ need to lie within $\mathcal{O}(3 \%)$ of the resonant peak for the annihilation to be strong enough. For completely decoupled DM multiplet the resonant condition is relaxed and needs to be satisfied to $\mathcal{O}(5 \%)$.

In Fig. 3.10 (upper panel) we show the spectrum for the lower mass solution and radiative DM multiplet splitting. We see that the quark partners of the lighter generations are heavier than the partners of the third generation quarks. Similarly, the FGBs that couple more strongly to the first two generations are typically heavier than the ones that couple preferably to the third generation. The couplings of the lightest FGB to the light quarks have the form $\hat{\mathcal{G}}_{L}^{u} \simeq \hat{\mathcal{G}}_{R}^{u} \simeq \hat{\mathcal{G}}_{L}^{d} \simeq \hat{\mathcal{G}}_{R}^{d} \propto \lambda^{8}$, where the relative corrections to this relation are below the percent level. This means that the lightest FGB couples to the light quarks vectorially, $\hat{\mathcal{G}}_{A}^{u, d} \ll \hat{\mathcal{G}}_{V}^{u, d}$, to a very good approximation. The same is true for the majority of parameter-space points passing flavor constraints.

The largest effects in flavor physics are in the mixing observables, the mass splittings $\Delta m_{d, s}$ in $B_{d}-\bar{B}_{d}$ and $B_{s}-\bar{B}_{s}$ systems, respectively, and the mass splitting in the $K-\bar{K}$ mixing, $\Delta m_{K}$, and the related CP violating parameter $\epsilon_{K}$. The pulls in $b \rightarrow s \gamma$ and $B_{d}-\bar{B}_{d}$ mixing are due to the fact that the measurements agree with the SM prediction only at $1-\sigma$ level and the contribution to them from new states is very small.

"Benchmark 2" is an example of a generic parameter-space region, but towards the upper end of the perturbatively allowed region. The DM has a mass $m_{\chi_{1}} \simeq 4.5 \mathrm{TeV}$, while the heavier states in the DM multiplet have masses $120 \mathrm{GeV}$ and $150 \mathrm{GeV}$ above $m_{\chi_{1}}$ (for the case of only radiative mass splitting). The lightest exotic quark is the top partner with mass $m_{t^{\prime}} \simeq 1.7 \mathrm{TeV}$, while the mass of the lightest FGB is $m_{A^{24}} \simeq 9.2 \mathrm{TeV}$. 


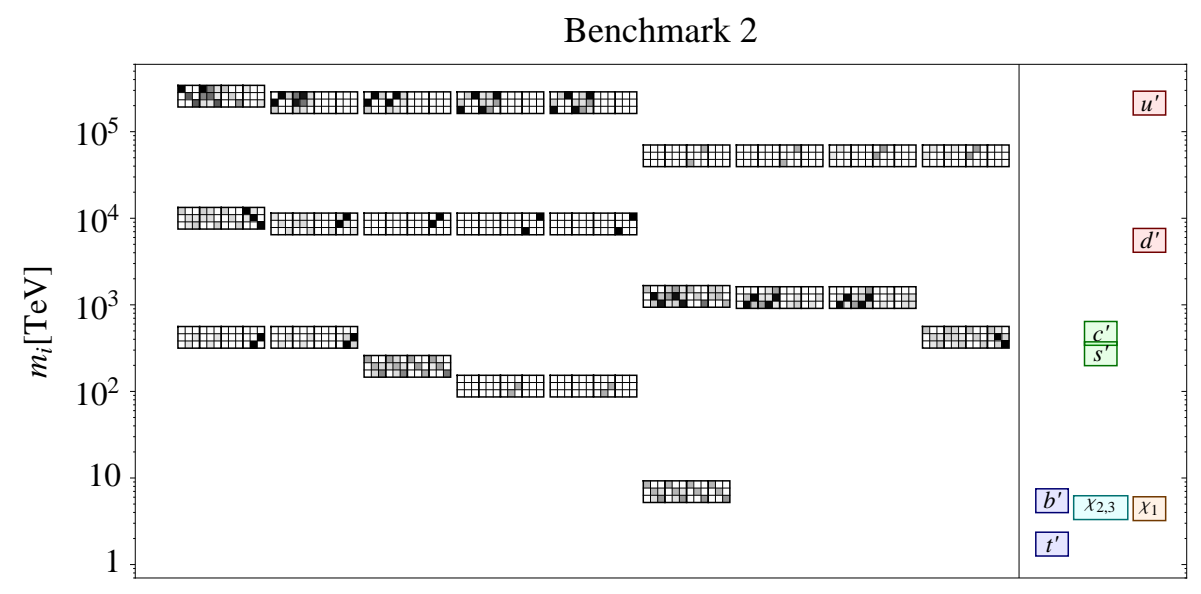

$M_{u}=980 . \mathrm{GeV}, \lambda_{u}=1 ., \lambda_{u}^{\prime}=0.79, M_{d}=360 . \mathrm{GeV}, \lambda_{d}=0.146, \lambda_{d}^{\prime}=0.91, g_{Q}=1.93, g_{U}=0.47, g_{D}=3.4$
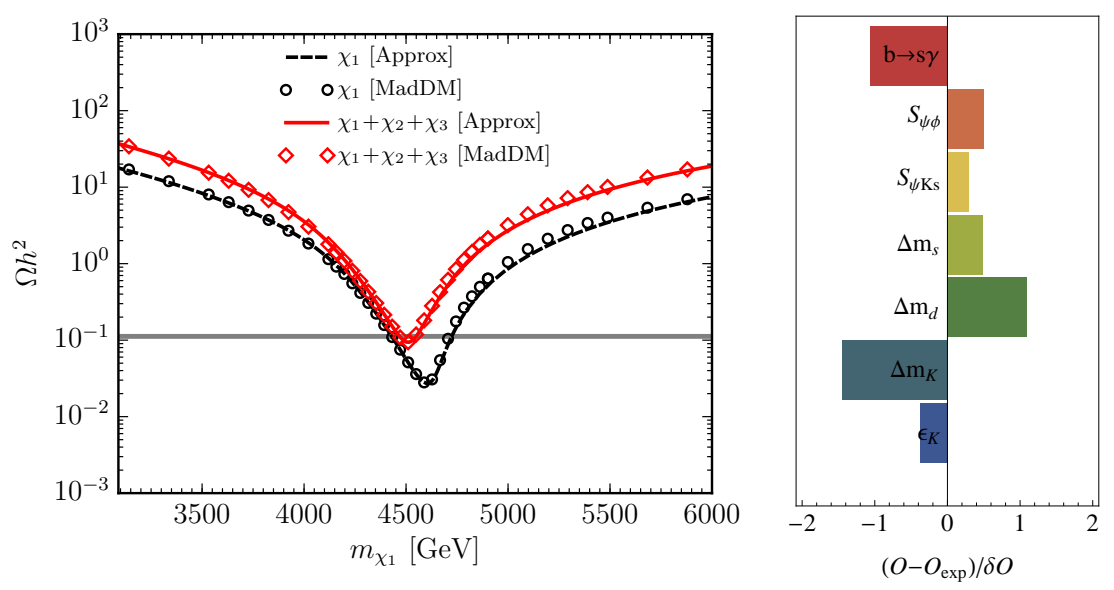

Figure 3.11: Same as Fig. 3.10 for benchmark 2. 


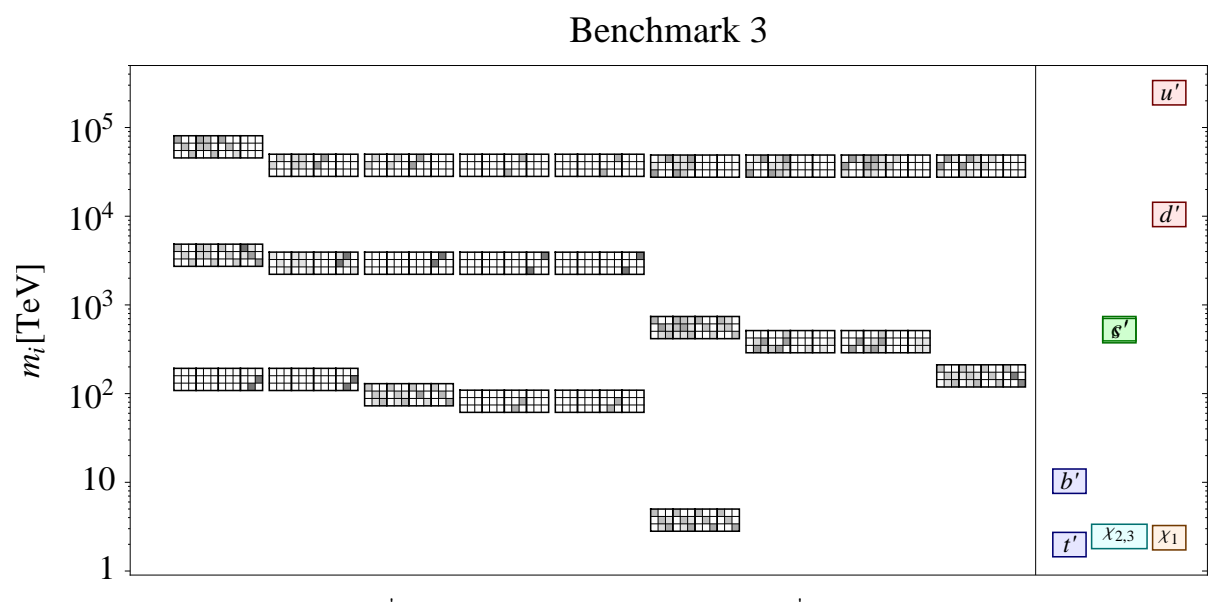

$M_{u}=1210 . \mathrm{GeV}, \lambda_{u}=1 ., \lambda_{u}^{\prime}=1.3, M_{d}=293 . \mathrm{GeV}, \lambda_{d}=0.36, \lambda_{d}^{\prime}=1.24, g_{Q}=0.45, g_{U}=0.46, g_{D}=0.82$
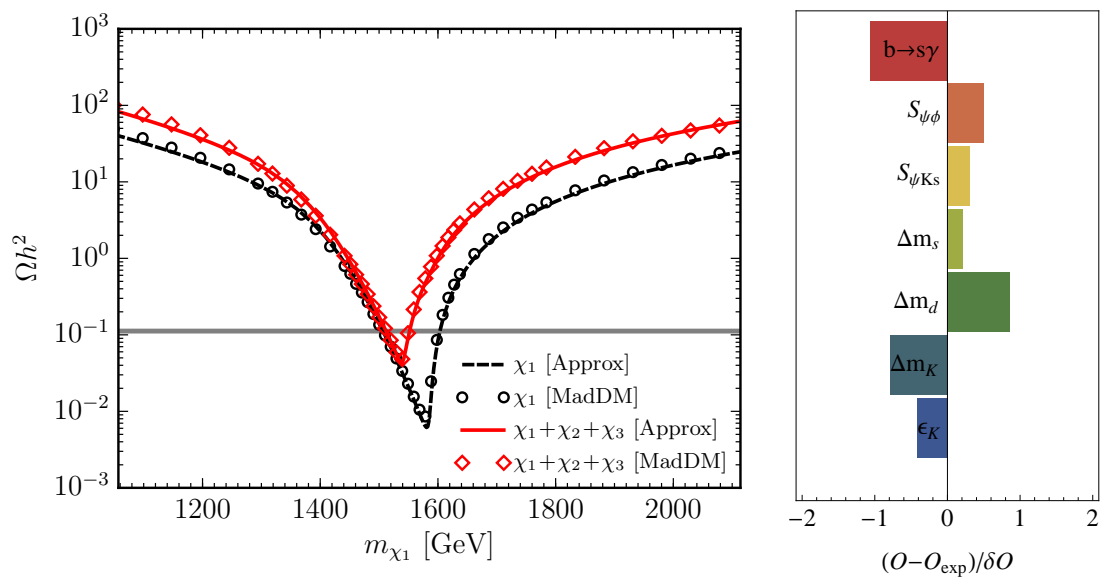

Figure 3.12: Same as Fig. 3.10 for benchmark 3.

For such high DM masses it is barely possible to obtain the correct relic abundance (see the lower left panel in Fig. 3.11). Therefore, the DM mass is finely tuned to be exactly on the resonant peak (see the lower left panel in Fig. 3.11), so $m_{\chi_{i}} \simeq m_{A^{24}} / 2$. Because of the high masses of the NP states the direct searches (direct DM detection, $t^{\prime}$ searches and dijet resonance searches) as well as the indirect flavor constraints are easily avoided, although $K-\bar{K}$ mixing does receive non-negligible contributions.

Also in this case, the couplings of the lightest FGB to the light quarks have the form $\hat{\mathcal{G}}_{L}^{u} \simeq \hat{\mathcal{G}}_{R}^{u} \simeq \hat{\mathcal{G}}_{L}^{d} \simeq \hat{\mathcal{G}}_{R}^{d} \propto \lambda^{8}$, so the couplings of the lightest FGB to quarks are to a good extent vectorial. 


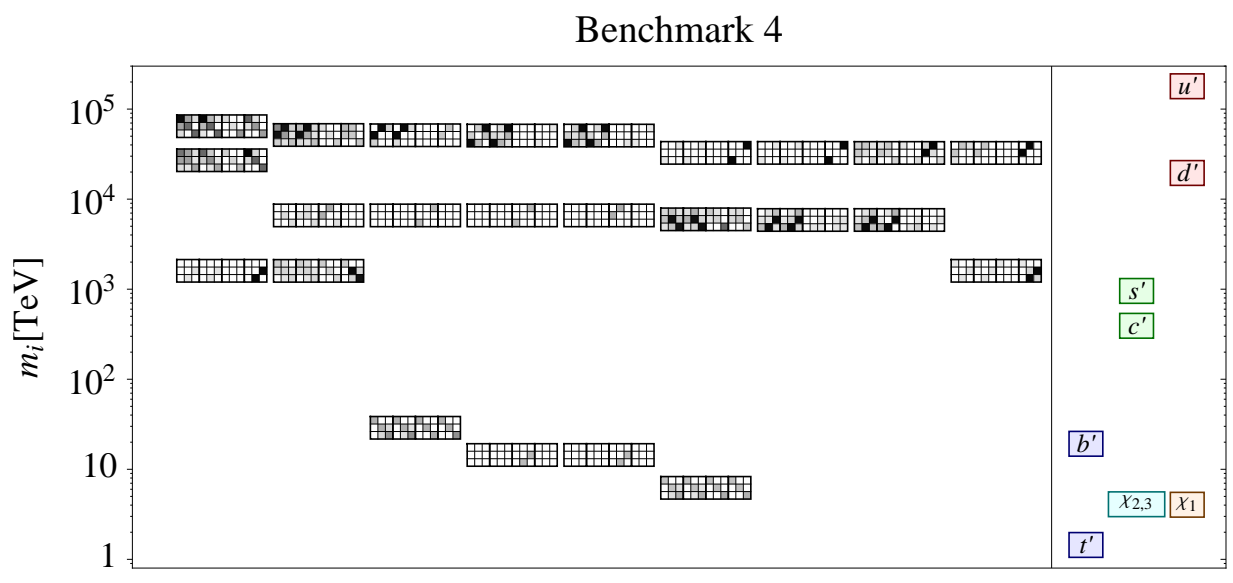

$M_{u}=830 . \mathrm{GeV}, \lambda_{u}=1 ., \lambda_{u}^{\prime}=3.5, M_{d}=1920 . \mathrm{GeV}, \lambda_{d}=0.096, \lambda_{d}^{\prime}=0.48, g_{Q}=1.89, g_{U}=0.312, g_{D}=1.93$
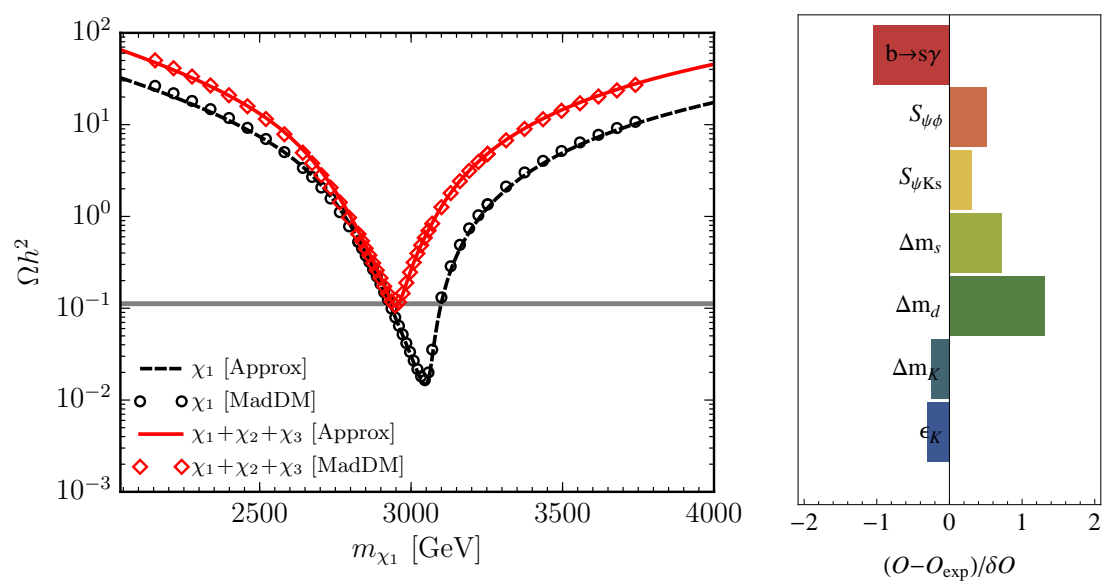

Figure 3.13: Same as Fig. 3.10 for benchmark 4.

"Benchmark 3" is an example of a generic parameter space in which all the couplings of the model are well below the pertubativity bounds. The lightest FGB has a mass $m_{A^{24}} \simeq 5 \mathrm{TeV}$ while all other FGBs have masses above $100 \mathrm{TeV}$. The lightest partner quark is $t^{\prime}$ with mass $m_{t^{\prime}} \simeq 2 \mathrm{TeV}$. The DM states have masses $m_{\chi_{1}} \simeq 2.4 \mathrm{TeV}$ and $m_{\chi_{2}} \simeq m_{\chi_{3}}=2.5 \mathrm{TeV}$ (for radiative mass splitting). All direct experimental constraints as well indirect flavor bounds are easily satisfied in this case. For radiatively DM mass splitting the cosmological constraints are the most constraining. In particular, requir$\operatorname{ing}$ small enough $\tau_{\chi_{2,3}}$ (or equivalently large enough $\Delta m_{21,31}$ ) typically imposes a lower bound on $g_{U}$. 
"Benchmark 4" is an example of the case in which the next-to-lightest FGBs have masses not too far from the lightest FGB. In the benchmark point the lightest FGB has a mass $m_{A^{24}} \simeq 8.3 \mathrm{TeV}$, while the next to lightest FGBs have masses $m_{A^{23}} \simeq m_{A^{22}} \simeq$ $19 \mathrm{TeV}, m_{A^{21}} \simeq 39 \mathrm{TeV}$. In this case the deviations from the $\hat{\mathcal{G}}_{L}^{u} \simeq \hat{\mathcal{G}}_{R}^{u} \simeq \hat{\mathcal{G}}_{L}^{d} \simeq \hat{\mathcal{G}}_{R}^{d} \propto \lambda^{8}$ relation for the lightest FGB coupling to quarks are of $\mathcal{O}(10 \%)$. Nonetheless, this does not have a significant effect on the computation of the DM relic abundance. This is demonstrated in Fig. 3.13 bottom left panel where a comparison is shown between the relicabundance calculation neglecting (dashed lines, labelled "Approx") and including (full lines, using MadDM) flavor off-diagonal lightest FGB couplings and contributions of heavier FGBs. For a more detailed discussion of these effects see Appendix B.II.

The lightest quark partner is $t^{\prime}$ with mass $m_{t^{\prime}} \simeq 1.4 \mathrm{TeV}$, and is significantly lighter than all FGBs and also DM. The DM states are degenerate to a good approximation, with masses $m_{\chi_{1}}=4.1 \mathrm{TeV}$ and $m_{\chi_{2}} \simeq m_{\chi_{3}} \simeq 4.2 \mathrm{TeV}$. Because of the light $t^{\prime}$ the flavor constraints are nontrivial, and there are visible effects in $B_{d}$ and $B_{s}$ mixing observables.

\subsection{Chapter summary}

We investigated the possibility that DM is in a nontrivial representation of the continuous flavor group $\mathcal{G}_{F}^{\mathrm{SM}}=S U(3)_{Q} \times S U(3)_{U} \times S U(3)_{D}$. The two main results are that (i) one can have a viable model of DM where DM is stable because it is charged under $\mathcal{Z}_{3}^{\chi}$ - a discrete central subgroup of $\mathcal{G}_{F}^{\mathrm{SM}}$ and color $S U(3)$, and (ii) that the DM spectrum can be very non-MFV like, while all the low-energy constraints will appear MFV-like.

$\mathcal{Z}_{3}^{\chi}$ is exactly conserved in many models of flavor. For instance, it remains unbroken for MFV new physics. More generally, $\mathcal{Z}_{3}^{\chi}$ remains exact if the flavor group $\mathcal{G}_{F}^{\mathrm{SM}}$ is broken only by the vevs of scalar fields, or condensates, with zero flavor triality. Examples of zero flavor triality fields are scalars in bifundamental or in adjoint representations of the flavor $S U(3)^{\prime}$ s. The basic requirement for this set-up is that $\mathcal{G}_{F}^{\mathrm{SM}}$ is a 
good symmetry in the UV. This is achieved, if $\mathcal{G}_{F}^{\mathrm{SM}}$ is fully gauged in the UV, which is the possibility we explored. The DM is then stable because it is $\mathcal{Z}_{3}^{\chi}$ odd, while all SM fields are $\mathcal{Z}_{3}^{\chi}$ even.

We investigated two different types of flavored DM models: (i) models in which the leading interaction of the DM with the visible sector is through the flavored gauge bosons (FGBs), and (ii) models in which the contributions from the FGB exchanges are subleading.

As an example of the first type of models we considered a Dirac fermion DM that is in a fundamental representation of $S U(3)_{U}$. The relic abundance is fixed by the resonant DM annihilation to SM particles through the $s$-channel exchange of the lightest FGB. The DM is thus required to have a mass of about half of the lightest FGB's mass. This in turn implies that the FGBs cannot be arbitrarily heavy, but at most $\mathcal{O}(10 \mathrm{TeV})$. Such light FGBs are possible, if the masses of FGBs are inversely proportional to the corresponding quark masses. That is, if the FGBs that couple most strongly to light quarks are also the heaviest. To achieve this we adopted the model of Ref. [91] in which the inverse proportionality is achieved by introducing a set of quark partners also necessary to cancel gauge anomalies. The same quark partners also mix with the SM quarks and lead to the mass hierarchy of the SM quark masses.

The flavor and collider phenomenology of the model is very similar to the case where DM is not considered. The fact that the first-generation quark partners are the heaviest and that the spectrum is completely split, signals the non-MFV character of the model. However, the low-energy consequences are MFV-like (see Appendix B.I). The flavor constraints are satisfied even with FGBs and the top-quark partner, with masses potentially well below the $\mathrm{TeV}$ scale. The relevant direct collider searches are the searches for dijet resonances and $t^{\prime}$ searches. They exclude part of the available parameter space. Requiring that there is a thermal relic DM introduces new constraints. Because DM is part of a flavor multiplet the heavier DM states need to decay before 
big bang nucleosynthesis. In the case of radiatively-split fermionic DM this excludes a large part of the parameter space. The remaining points are mostly safe from directdetection bounds. The fact that the DM mass is related to the FGB mass by the requirement of almost resonant annihilation sets both lower and upper bounds on the DM mass. Requiring that the theory is perturbative also puts an upper bound on the DM mass, $m_{\chi_{1}} \lesssim 5 \mathrm{TeV}$. On the other hand, requiring that the FGBs satisfy flavor and direct constraints and that DM is simultaneously in accordance with cosmological constraints leads to a lower bound on the DM mass, $m_{\chi_{1}} \gtrsim 500 \mathrm{GeV}$. Improved bounds on dijet resonances at the LHC are expected to further strengthen this constraint (see Fig. 3.9).

We have also considered the possibility that the DM multiplet is split due to an extra source of flavor breaking. Also in this case, the correct relic abundance requires resonant annihilation. The DM mass is in thus still roughly equal to half of the mass of the lightest FGB. However, the heavier DM states decay well before big bang nucleosynthesis so that a much wider range of DM masses is phenomenologically viable. In our scan this includes DM masses as light as $100 \mathrm{GeV}$ (with very small couplings to FGBs) and up to $10 \mathrm{TeV}$.

A possible signal of the gauged flavor model with fermionic DM at the LHC are mono-jets, where the lightest FGB is produced associated with initial state radiation and decays to $\chi_{1}$ pairs. The $\chi_{1} s$ are expected to be non-relativistic in the lightest FGB's rest frame, as their combined mass needs to be close to the FGB mass to fulfill the resonance condition for relic DM abundance. In the event that such a signal would eventually emerge, the corresponding dijet-resonance signal is generically also expected in the model. A final possibility in the case of radiatively split DM mass spectrum is that some of the lightest FGBs decay to slow-moving $\chi_{2,3}$. They in turn decay within the detector, leaving (highly) displaced vertices, isolated hits in the calorimeter or in the muon chambers. Unfortunately, in most of the parameter space $\chi_{2,3}$ are expected to decay well outside the detectors, see Fig. 3.5, leaving mono-jets as the only signal. 
In the second type of models, where FGB exchanges give only subleading contributions, the only visible consequence of the flavor dynamics on the DM is that DM is stable. The DM mass and the mass of the lightest FGB are no longer connected. We show this in the example of scalar flavored DM, in which the dominant interactions with the visible sector are through the Higgs portal operator. In this case the phenomenology of the DM is to a very good approximation the same as in the Higgs-portal scalar DM, while the dynamics of FGBs and quark partners is unrelated to DM.

In short, we have shown, using an explicit renormalizable model, that it is possible for flavored DM to be a thermal relic. The considered model is not the only choice. One could consider DM in other representations of $\mathcal{G}_{F}^{\mathrm{SM}}$. Our analysis can be extended also in other ways: for instance, by enlarging the global symmetry as in Ref. [24] and subsequently gauging it. For instance, with our field content the global group is $\mathcal{G}_{F}^{\mathrm{SM}} \times$ $S U(3)_{\chi}$, where $\chi$ is in the fundamental of $S U(3)_{\chi}$. In our work we have identified $S U(3)_{\chi}$ with $S U(3)_{U}$, but other choices could be made. Yet another possibility is to gauge only part of $\mathcal{G}_{F}^{\mathrm{SM}}$, for instance a $U(2)^{3} \subset \mathcal{G}_{F}^{\mathrm{SM}}$. Note that for fermionic DM, $\mathcal{Z}_{3}$ is part of an accidental $U(1)_{\chi}$ acting in the dark sector. The $U(1)_{\chi}$ can be broken by the dimension-7 operator $L H \chi \chi \chi$, but is exact in our renormalizable model. It can in principle be gauged and lead to additional phenomenology. If DM is a scalar, $U(1)_{\chi}$ can be broken already at the renormalizable level, leaving only $\mathcal{Z}_{3}$ exact. 


\section{Chapter 4}

\section{Nonstandard Yukawa couplings and}

\section{Higgs portal dark matter}

\subsection{Introduction}

In Higgs-portal models [147-156] of dark matter (DM) the Higgs is usually assumed to be completely Standard Model (SM) like apart from its couplings to DM. Experimentally, only the couplings of the Higgs to the heaviest particles of the SM are currently well constrained. The couplings to gauge bosons are found to be in agreement with the SM predictions at the $\mathcal{O}(20 \%)$ level, while the constraints on the couplings to third-generation fermions are somewhat weaker [157, 158]. Much less is known experimentally about the couplings of the Higgs to the first two generations of fermions. The couplings to $u, d, s$, and $c$ quarks could be as large as the SM bottom Yukawa coupling or be absent altogether [159-162]. The Higgs couplings to top and bottom quarks will be quite well known by the end of the high-luminosity LHC run. Some progress is also expected on the measurements of Higgs couplings to charm and strange quarks [159-161].

Large $u-, d-$, and $s$-quark Yukawa couplings, comparable in size to the $b$-quark 
Yukawa, generically require fine-tuning. A large Yukawa coupling implies a large contribution to the quark mass from the Higgs vacuum expectation value (vev). This would then need to be cancelled by a different contribution to the $u-, d-$, and $s$-quark masses, unrelated to the Higgs vev. The opposite limit, where the observed Higgs does not couple to the light quarks at all is easier to entertain. It simply requires a separate source of the light-quark masses (for an extreme example see, e.g., [163]).

Modified light-quark Yukawa couplings could, in principle, have important implications for DM phenomenology. In this article we investigate how the Higgs-portal DM predictions change if the Higgs couplings to the light quarks differ from the SM expectations. We first allow for an arbitrary flavor structure of the Higgs Yukawa couplings, only requiring that they satisfy the current experimental bounds. In Section 4.2 we derive the implications for direct DM detection, indirect DM detection and the collider searches. We show that vanishing couplings of the Higgs to light quarks only have a relatively small impact on these observables. Saturating the loose current bounds on the light-quark Yukawa couplings would, on the other hand, lead to drastically enhanced scattering cross sections on nuclei while leaving the relic density and annihilation cross sections

Clearly, an enhancement of the light-quark Yukawas by factors of $\mathcal{O}(100)$ or more, as allowed by current data, requires considerable fine tuning of the quark-mass terms and hence seems quite unlikely. In Section 4.3 we, therefore, explore the deviations in the Higgs Yukawa couplings for a number of beyond-the-SM scenarios and flavor models. This leads to more realistic expectations as to how large the deviations in the direct DM detection rates can be due to the poorly known Higgs couplings to the light quarks. Note that we assume the DM to be a flavor singlet and that the new flavor structure of the interactions with the visible sector is only due to the modification of the SM Higgs couplings. DM that is in a nontrivial flavor multiplet has been investigated in $[21,23-26,29,39,94,96,97,164,165]$, while our study is closer in spirit to the work 
in $[28,93,166]$ where the flavor dependence of the DM signals for flavor-singlet DM has been explored.

A somewhat surprising result of our investigation is that, if DM is discovered and turns out to be a thermal relic predominantly interacting through a Higgs portal, it could be used to constrain the light-quark Yukawa couplings. This is discussed in more detail in Section 4.4.

We summarize the results of this chapter in Section 4.5.

\subsection{Higgs portal with non-trivial flavor structure}

We assume that DM and the SM fields are the only light degrees of freedom. The remaining new physics (NP) particles can be integrated out so that one can use an Effective Field Theory (EFT) approach. The couplings of DM to the SM are given by the Higgs-portal Lagrangian

$$
\mathcal{L}_{\chi}= \begin{cases}g_{\chi} \chi^{\dagger} \chi H^{\dagger} H, & \text { scalar DM; } \\ g_{\chi} \frac{1}{\Lambda} \bar{\chi} \chi H^{\dagger} H+i \tilde{g}_{\chi} \frac{1}{\Lambda} \bar{\chi} \gamma_{5} \chi H^{\dagger} H, & \text { fermion DM; } \\ \frac{g_{\chi}}{2} \chi^{\mu} \chi_{\mu} H^{\dagger} H, & \text { vector DM. }\end{cases}
$$

Above, the fermion DM can be either a Dirac or Majorana fermion (in either case we use four component notation). After electroweak symmetry breaking (EWSB) we have

$$
H^{\dagger} H=\frac{1}{2}\left(v_{W}^{2}+2 v_{W} h+h^{2}\right)
$$

where $v_{W}=246 \mathrm{GeV}$ is the vacuum expectation value (vev) of the Higgs field. The above interactions therefore lead to annihilation of DM into both single Higgs, $\chi \bar{\chi} \rightarrow h$, and double Higgs, $\chi \bar{\chi} \rightarrow h h$, final states.

The Higgs-portal operator for fermionic DM has mass dimension five and is sup- 
pressed by the new physics scale $\Lambda$. The Higgs-portal interaction for fermionic DM can also be re-written as $\mathcal{L}_{\chi}=\left(g_{\chi}+i \tilde{g}_{\chi}\right) \bar{\chi}_{L} \chi_{R} H^{\dagger} H / \Lambda+$ h.c.. For $\tilde{g}_{\chi} \neq 0$ the interaction is thus both $P$ - and $C P$-violating. The interaction for vector DM is most probably also due to a higher-dimensional operator in the full theory. For instance, if $\chi_{\mu}$ arises from a spontaneously broken gauge symmetry in the dark sector, then $g_{\chi} \sim v_{D}^{2} / \Lambda^{2}$, where $v_{D}$ is the vev of the field that breaks the dark sector gauge invariance, while $\Lambda$ is the mass of the mediator between DM and the Higgs.

The relevant terms, after EWSB, in the effective Lagrangian for the Higgs couplings to the SM particles are given by

$$
\begin{aligned}
\mathcal{L}_{\text {eff }} & =-\kappa_{q} \frac{m_{q}}{v_{W}} \bar{q} q-\kappa_{\ell} \frac{m_{\ell}}{v_{W}} \bar{\ell} \ell h+\kappa_{V}\left(\frac{2 m_{W}^{2}}{v_{W}} W^{+\mu} W_{\mu}^{-}+\frac{m_{Z}^{2}}{v_{W}} Z^{\mu} Z_{\mu}\right) h \\
& -\kappa_{\lambda} \frac{m_{h}^{2}}{2 v_{W}} h^{3}+\kappa_{g}^{\mathrm{NP}} \frac{\alpha_{s}}{12 \pi v_{W}} h G_{\mu \nu}^{a} G^{a \mu \nu}
\end{aligned}
$$

where the $\kappa_{i}$ are real. A sum over the SM quarks, $q=u, d, s, c, b, t$, and charged leptons, $\ell=e, \mu, \tau$ is implied, and we have assumed custodial symmetry. The $h \rightarrow \gamma \gamma$ coupling is not relevant for DM phenomenology, since its effects are suppressed compared to the Higgs couplings to gluons. ${ }^{1}$ The couplings are normalized such that $\kappa_{q}=\kappa_{\ell}=\kappa_{V}=$ $\kappa_{\lambda}=1$ correspond to the SM. The experimental constraints on the couplings of the light quarks to the Higgs, obtained from a global fit to current data, are $\left|\kappa_{u}\right|<0.98 m_{b} / m_{u}$, $\left|\kappa_{d}\right|<0.93 m_{b} / m_{d},\left|\kappa_{s}\right|<0.70 m_{b} / m_{d}$, where only one of the light Yukawa couplings was left to float in the fit, while all the other Higgs couplings are set to the SM values [159]. Higgs couplings to the light quarks of a size comparable to the coupling to the $b$ quark are thus still allowed. In (4.3) we do not allow for flavor violating Higgs couplings, since these are already tightly constrained from both Higgs decays and low-energy observables [167-170].

\footnotetext{
${ }^{1}$ It could be relevant for direct detection if the scattering on electrons dominates. This requires very light DM, of order the electron mass. Such light Higgs-portal DM is excluded by the constraints on the Higgs invisible branching ratio.
} 
In the SM, keeping the Higgs on shell, the $h G G$ coupling arises predominantly from the one-loop top-quark contribution. The $\kappa_{g}^{\mathrm{NP}}$ in (4.3) encodes only the potential NP contributions, and vanishes in the SM. In the global fits a parameter $\kappa_{g}$ is introduced that gives the total $h \rightarrow g g$ amplitude, including the SM contributions $[157,158]$. We have (see, e.g., [171])

$$
\kappa_{g} \simeq 1.03 \kappa_{t}+\kappa_{g}^{\mathrm{NP}}
$$

At present, significant $C P$-violating Higgs couplings to fermions and gluons are still allowed experimentally (see, e.g., [172]), so we also discuss their effect on the Higgs interactions with DM:

$$
\mathcal{L}_{\mathrm{eff}, \mathrm{CPV}}=-i \tilde{\kappa}_{q} \frac{m_{q}}{v_{W}} \bar{q} \gamma_{5} q h-i \tilde{\kappa}_{\ell} \frac{m_{\ell}}{v_{W}} \bar{\ell} \gamma_{5} \ell h+\tilde{\kappa}_{g}^{\mathrm{NP}} \frac{\alpha_{s}}{8 \pi v_{W}} h G_{\mu \nu}^{a} \widetilde{G}^{a \mu \nu}
$$

Here, the $\tilde{\kappa}_{i}$ are real parameters; in the SM, we have $\tilde{\kappa}_{i}=0$. Moreover, $G_{\mu \nu}^{a}$ is the gluon field-strength tensor and $\widetilde{G}^{a, \mu \nu}=\frac{1}{2} \epsilon^{\mu \nu \alpha \beta} G_{\alpha \beta}^{a}$ its dual. The normalization of the $h G \tilde{G}$ term is chosen such that integrating out the top at one loop one obtains $\tilde{\kappa}_{g}=\tilde{\kappa}_{t}+\tilde{\kappa}_{g}^{N P}$. Accordingly, we have $\operatorname{Br}(h \rightarrow g g) \propto \kappa_{g}^{2}+\left(3 \tilde{\kappa}_{g} / 2\right)^{2}$. The $C P$-violating couplings of the Higgs to $Z Z$ and $W W$ are already well constrained, and we thus set them to zero. In the numerical analysis below, we will also assume $\tilde{\kappa}_{i}=0$, for simplicity.

The modified Higgs couplings change the usual Higgs-portal predictions for DM annihilation rates, the relic abundance, and direct detection rates. In the following, we discuss these modifications in detail.

\subsubsection{Annihilation cross sections}

The dominant DM annihilation cross sections in the Higgs-portal models are $\chi \bar{\chi} \rightarrow$ $b \bar{b}, W^{+} W^{-}, Z Z, t \bar{t}$, and $\chi \bar{\chi} \rightarrow h h$. The first four proceed through the $s$-channel Higgs exchange, while $\chi \bar{\chi} \rightarrow h h$ receives additional contributions from $t$ - and $u$-channel $\chi$ 

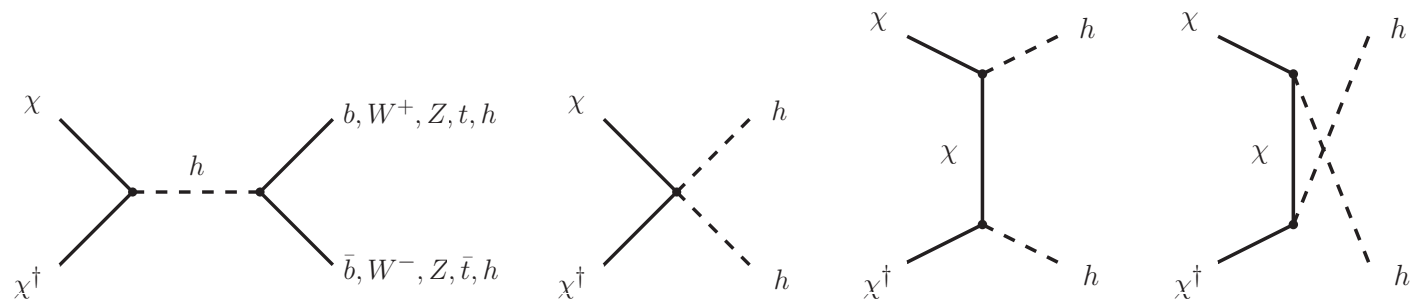

Figure 4.1: DM annihilation channels in the Higgs-portal models.

exchange as well as from the four-point contact interaction (cf. Fig. 4.1). The $\chi \bar{\chi} \rightarrow b \bar{b}$ channel is only relevant if the other channels are not kinematically allowed, i.e. for light DM masses, $m_{\chi}<m_{W}$.

The $\chi \bar{\chi} \rightarrow \bar{b} b$ annihilation cross section assuming SM Higgs couplings is given for scalar $(S)$, Dirac fermion $(D F)$, and vector $(V)$ DM by

$$
\begin{aligned}
\left(\sigma_{b \bar{b}}^{S} v_{\mathrm{rel}}\right)_{S M} & =\frac{N_{c}}{4 \pi} \frac{g_{\chi}^{2} m_{b}^{2} \beta_{b}^{3}}{\left(s-m_{h}^{2}\right)^{2}+m_{h}^{2} \Gamma_{h}^{2}}, \\
\left(\sigma_{b \bar{b}}^{D F} v_{\text {rel }}\right)_{S M} & =\frac{N_{c}}{8 \pi} \frac{m_{b}^{2}}{\Lambda^{2}} \frac{g_{\chi}^{2}\left(s-4 m_{\chi}^{2}\right)+\tilde{g}_{\chi}^{2} s}{\left(s-m_{h}^{2}\right)^{2}+m_{h}^{2} \Gamma_{h}^{2}} \beta_{b}^{3}, \\
\left(\sigma_{b \bar{b}}^{V} v_{\mathrm{rel}}\right)_{S M} & =\frac{N_{c}}{9} \frac{g_{\chi}^{2}}{16 \pi} \frac{m_{b}^{2}}{m_{\chi}^{4}} \beta_{b}^{3}\left(1-r_{\chi}+\frac{3}{4} r_{\chi}^{2}\right) \frac{s^{2}}{\left(s-m_{h}^{2}\right)^{2}+m_{h}^{2} \Gamma_{h}^{2}}
\end{aligned}
$$

where, here and below, $\sqrt{s}$ is the center-of-mass energy, $r_{k}=4 m_{k}^{2} / s, \beta_{k}=\sqrt{1-r_{k}}$ is the velocity of particle $k$, and $v_{\text {rel }}=2 \beta_{\chi}$ is the relative velocity of the DM particles. If the Higgs coupling to the $b$-quarks differs from the SM value, the annihilation cross section is rescaled as

$$
\sigma_{b \bar{b}}=\left(\kappa_{b}^{2}+\tilde{\kappa}_{b}^{2} / \beta_{b}^{2}\right) \sigma_{b \bar{b}}^{\mathrm{SM}} .
$$

The annihilation cross sections $\sigma_{f \bar{f}}$ to the other fermions are obtained with the obvious replacement $b \rightarrow f$ in the above expressions. Since the Higgs couplings to the light quarks are poorly constrained experimentally, the DM annihilation to two light quarks can be comparable to $\chi \bar{\chi} \rightarrow b \bar{b}$ and can be important for light DM, $m_{\chi}<m_{W}$. 
For heavy DM, $m_{\chi}>m_{W}$, the annihilation cross-sections into a pair of $W$ or $Z$ bosons are

$$
\sigma_{V V}=\kappa_{V}^{2} \sigma_{V V}^{\mathrm{SM}},
$$

$V=W, Z$. The annihilation cross sections assuming the SM Higgs couplings to $W$ are given by

$$
\begin{aligned}
\left(\sigma_{W W}^{S} v_{\mathrm{rel}}\right)_{\mathrm{SM}} & =\frac{g_{\chi}^{2}}{8 \pi} \beta_{W}\left(1-r_{W}+\frac{3}{4} r_{W}^{2}\right) \frac{s}{\left(s-m_{h}^{2}\right)^{2}+m_{h}^{2} \Gamma_{h}^{2}} \\
\left(\sigma_{W W}^{D F} v_{\mathrm{rel}}\right)_{\mathrm{SM}} & =\frac{1}{16 \pi \Lambda^{2}} \beta_{W}\left(1-r_{W}+\frac{3}{4} r_{W}^{2}\right) \frac{s\left[g_{\chi}^{2}\left(s-4 m_{\chi}^{2}\right)+\tilde{g}_{\chi}^{2} s\right]}{\left(s-m_{h}^{2}\right)^{2}+m_{h}^{2} \Gamma_{h}^{2}} \\
\left(\sigma_{W W}^{V} v_{\mathrm{rel}}\right)_{\mathrm{SM}} & =\frac{g_{\chi}^{2}}{288 \pi} \frac{s}{m_{\chi}^{4}} \beta_{W}\left(1-r_{W}+\frac{3}{4} r_{W}^{2}\right)\left(1-r_{\chi}+\frac{3}{4} r_{\chi}^{2}\right) \frac{s^{2}}{\left(s-m_{h}^{2}\right)^{2}+m_{h}^{2} \Gamma_{h}^{2}}
\end{aligned}
$$

for scalar, Dirac fermion, and vector $\mathrm{DM}$, respectively. The $\chi \bar{\chi} \rightarrow Z Z$ annihilation cross section is obtained by replacing $W \rightarrow Z$, and multiplying all expressions by an extra factor of $1 / 2$ since one has two indistinguishable particles in the final state.

The $\chi \bar{\chi} \rightarrow h h$ annihilation cross sections for scalar, Dirac fermion, and vector DM, are given by

$$
\begin{aligned}
\sigma_{h h}^{S} v_{\text {rel }}= & \frac{\beta_{h} g_{\chi}^{2}}{64 \pi m_{\chi}^{2}}\left[1+\frac{3 \kappa_{\lambda} M_{h}^{2}}{4 m_{\chi}^{2}-M_{h}^{2}}-\frac{2 v_{W}^{2} g_{\chi}}{M_{h}^{2}-2 m_{\chi}^{2}}\right]^{2} \\
\sigma_{h h}^{D F} v_{\text {rel }}= & \frac{\beta_{h}\left(\tilde{g}_{\chi}^{2}+g_{\chi}^{2} \beta_{\chi}^{2}\right)}{32 \pi \Lambda^{2}}\left[1+\frac{3 \kappa_{\lambda} M_{h}^{2}}{4 m_{\chi}^{2}-M_{h}^{2}}+\frac{4 g_{\chi} m_{\chi} v_{W}^{2}}{\Lambda\left(2 m_{\chi}^{2}-M_{h}^{2}\right)}\right]^{2} \\
\sigma_{h h}^{V} v_{\text {rel }}=\frac{\beta_{h}}{576 \pi m_{\chi}^{2}} & {\left[3 g_{\chi}^{2}\left(\frac{3 \kappa_{\lambda} M_{h}^{2}}{4 m_{\chi}^{2}-M_{h}^{2}}+1\right)^{2}+\frac{4 g_{\chi}^{4} v_{W}^{4}}{\left(2 m_{\chi}^{2}-M_{h}^{2}\right)^{2}}\left(6-\frac{4 M_{h}^{2}}{m_{\chi}^{2}}+\frac{M_{h}^{4}}{m_{\chi}^{4}}\right)\right.} \\
& \left.+\frac{16 g_{\chi}^{3} v_{W}^{2}}{2 m_{\chi}^{2}-M_{h}^{2}}\left(\frac{3 \kappa_{\lambda} M_{h}^{2}}{4 m_{\chi}^{2}-M_{h}^{2}}+1\right)\left(1-\frac{M_{h}^{2}}{4 m_{\chi}^{2}}\right)\right] .
\end{aligned}
$$

In this result we display only the leading terms in the expansion in powers of $\beta_{\chi}$. The 

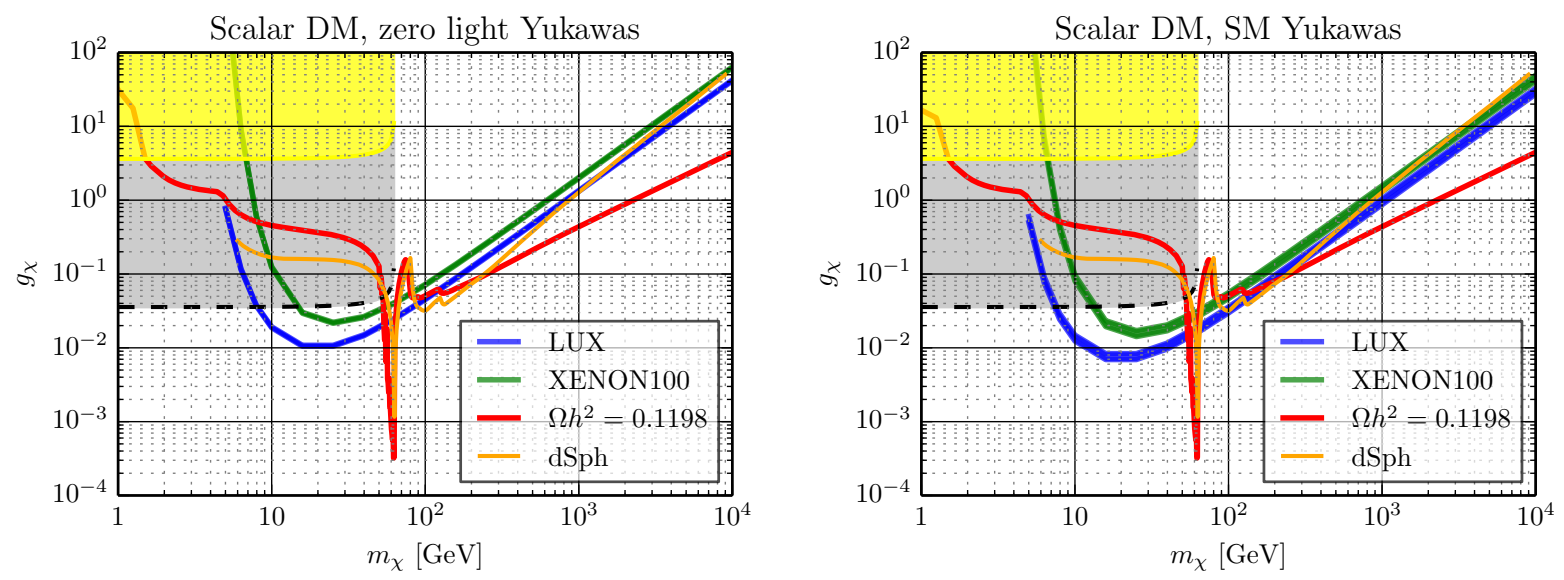

Figure 4.2: Bounds from LUX (blue band), XENON100 (green band) and the invisible Higgs decay width (black dashed line and grey region) on the Higgs-portal coupling $g_{\chi}$ for scalar DM, assuming vanishing (left) and SM (right) Yukawa couplings to $u, d$, $s$ quarks. The red line denotes $g_{\chi}$ as a function of DM mass, $m_{\chi}$, for which the correct relic abundance is obtained, while $g_{\chi}$ in the yellow region leads to non-perturbatively large Higgs decay width, $\Gamma_{h}>m_{h}$, and is excluded. Constraints from Fermi-LAT searches for DM annihilation in dwarf spheroidal galaxies are denoted by the orange band.

contribution of the $s$-channel Higgs exchange diagram is proportional to the rescaling of the trilinear Higgs coupling, $\kappa_{\lambda}$. The latter is completely unknown experimentally, at present, but can be measured to $\mathcal{O}(20 \%)$ at the end of the LHC [173,174]. Since we are mostly interested in the effects of flavor modifications we will set it to the SM value, $\kappa_{\lambda}=1$, in the numerics below. All cross sections for Majorana DM can be obtained by multiplying the corresponding Dirac DM cross sections by a factor of 4 .

\subsubsection{Relic abundance}

The DM relic abundance $\Omega_{D M}$ is proportional to $1 / \sigma v_{\text {rel }}$, where $\sigma$ is the annihilation cross section. Assuming that the DM in our scenario accounts for all of the observed relic density, the measured value $\Omega_{D M} h^{2}=0.1198(26)$ [55] fixes $g_{\chi}$ for a given value of $m_{\chi}$. The resulting constraint in the $m_{\chi}-g_{\chi}$ plane is denoted for the different cases 


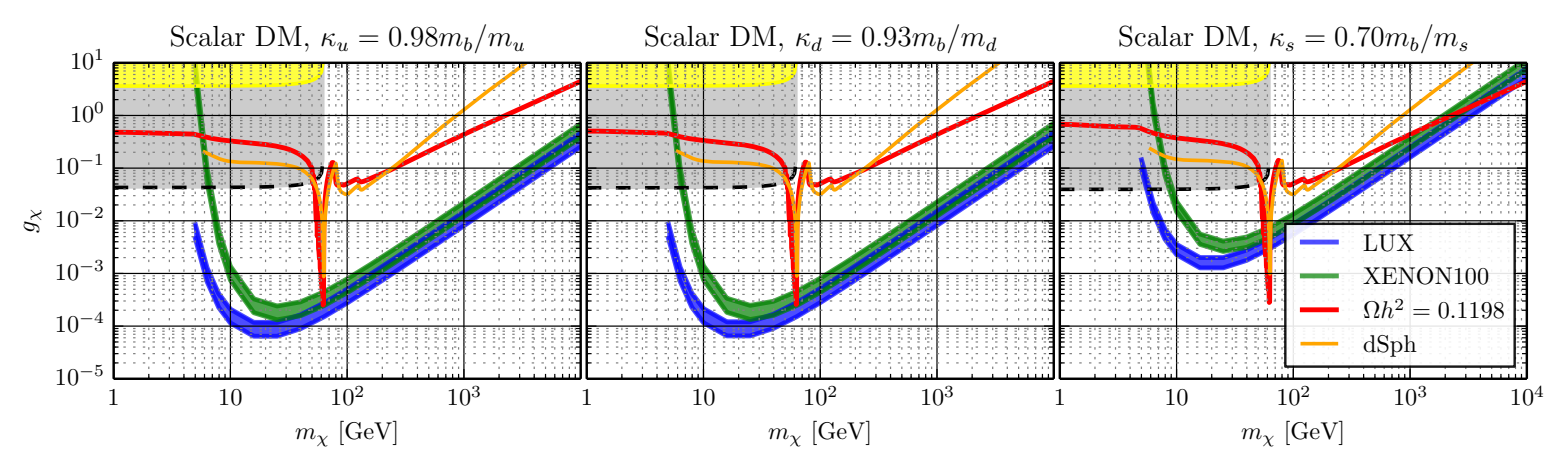

Figure 4.3: Bounds on the Higgs-portal coupling $g_{\chi}$ for scalar DM, assuming maximal allowed values for the Yukawa couplings to the $u, d, s$ quarks (left to right), keeping all the other couplings to their SM values. The color coding is the same as in Fig. 4.2.

by a red line in Figs. 4.2 to 4.9. In Fig. 4.2, we compare two limits of the Higgs portal for the scalar DM: the case where the Higgs does not couple to the light quarks at all (left panel) to the case where the Higgs has SM Yukawa couplings (right panel). The two relic abundance curves coincide apart from very light DM, with $m_{\chi}$ below the charm and tau threshold. If such light DM did not couple to the $u, d$ and $s$ quarks, this would result in noticeably reduced annihilation cross sections and, thus, in larger relic abundance. In both cases, the dominant annihilation process is still given by $\chi \bar{\chi} \rightarrow$ $h^{*} \rightarrow g g$. For very light DM the correct relic abundance is obtained only if the coupling of the Higgs to DM, $g_{\chi}$, is nonperturbatively large. The yellow regions in Fig. 4.2 denote the value of $g_{\chi}$ for which the total Higgs decay width would be larger than its mass, $\Gamma_{h}>m_{h}$, and are thus excluded.

The same comments apply to the case of vector DM, shown in Fig. 4.4, $C P$-conserving Dirac fermion DM, shown in Fig. 4.6, $C P$-violating Dirac fermion DM, shown in Fig. 4.8, and also for Majorana fermion DM. For light DM, $m_{\chi} \lesssim 30 \mathrm{GeV}$, the correct relic density requires a non-perturbatively large coupling $g_{\chi}$ so that the predictions should be taken only as $\mathcal{O}(1)$ estimates in that region. Note that all these non-perturbative regions are, in addition, excluded by bounds on the decay width of the Higgs into invisible final 

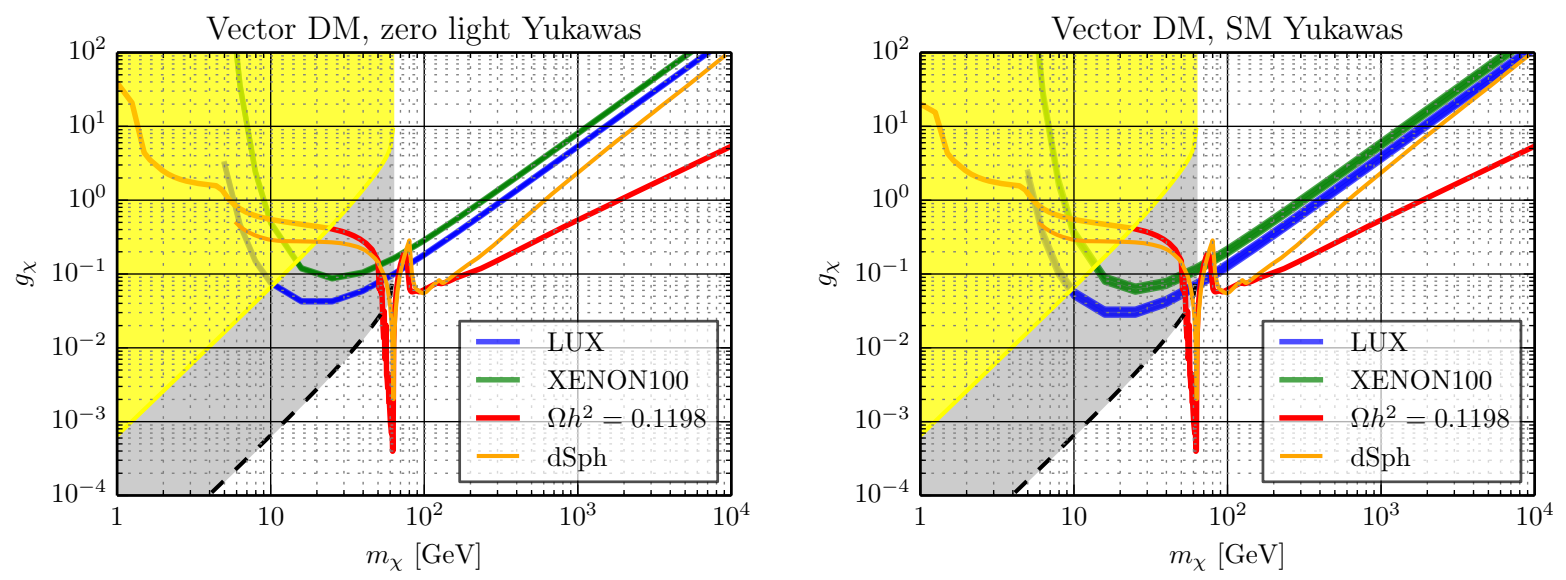

Figure 4.4: Bounds on the Higgs-portal coupling for vector DM, assuming vanishing (left) and SM (right) Yukawa couplings to $u, d, s$ quarks. The color coding is the same as in Fig. 4.2.

states (see the discussion in Section 4.2.3).

In Figs. 4.3, 4.5, 4.7, and 4.9, we show the relic abundance curves for $g_{\chi}$ as a function of $m_{\chi}$ for the case where the light Yukawa couplings saturate their upper experimental bound. The left panels show the case where $\kappa_{u}=0.98 m_{b} / m_{u}$ and all the other couplings at their SM values, the middle panels the case where $\kappa_{d}=0.93 m_{b} / m_{d}$, and the right panels the case where $\kappa_{s}=0.70 m_{b} / m_{s}$. In all of these cases the cross section for DM annihilation to light jets, $\sigma(\chi \bar{\chi} \rightarrow j j)$, coming from DM annihilating to light quarks, is comparable to the annihilation cross section to $b$-jets, $\sigma(\chi \bar{\chi} \rightarrow b \bar{b})$. For $m_{\chi} \lesssim m_{W}$ these are the two dominant annihilation modes. Since the annihilation cross sections to $b$-jets and light jets are comparable, the relic abundance curve show only a small change in $g_{\chi}$ when the $b$-quark threshold is reached. This should be compared with the case of the SM Yukawa couplings shown in the right panels of Figs. 4.2, 4.4, 4.6, and 4.8. In this case the $\chi \bar{\chi} \rightarrow j j$ annihilation is almost exclusively due to DM annihilating to two gluons, so that $\sigma(\chi \bar{\chi} \rightarrow j j) \ll \sigma(\chi \bar{\chi} \rightarrow b \bar{b})$, while the annihilation into two light quarks is negligible. For $m_{\chi} \lesssim m_{W}$ and SM Yukawas, the required $g_{\chi}$ is thus bigger by $30 \%-40 \%$ than in the case of light Yukawas at their present experimental 


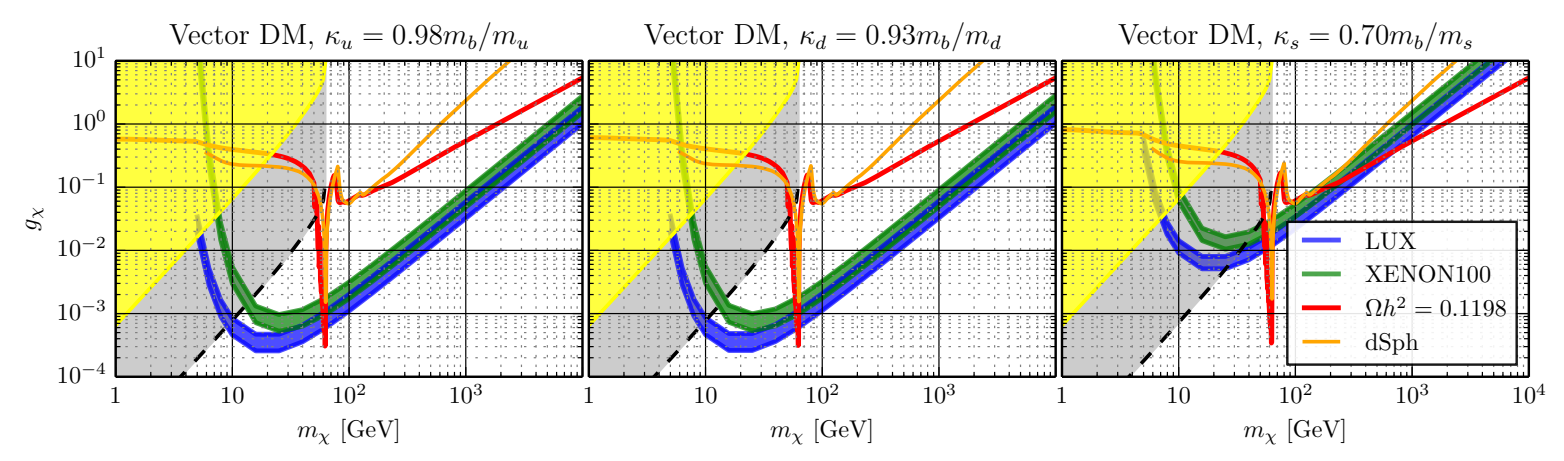

Figure 4.5: Bounds on the Higgs-portal coupling for vector DM, assuming maximal allowed values for the Yukawa couplings to the $u, d, s$ quarks (left to right), keeping all the other couplings to their SM values. The color coding is the same as in Fig. 4.2.

limits, and exhibits a significant jump below the $b$-quark threshold.

\subsubsection{Invisible decay width of the Higgs}

The bounds on the invisible decay width of the Higgs boson provide stringent constraints on Higgs-portal DM [153]. The partial $h \rightarrow \chi \bar{\chi}$ decay widths are given by

$$
\begin{aligned}
\Gamma_{\chi \chi}^{S} & =\frac{g_{\chi}^{2}}{16 \pi} \frac{v_{W}^{2}}{M_{h}} \beta_{\chi}, \\
\Gamma_{\chi \chi}^{D F} & =\frac{g_{\chi}^{2}}{8 \pi} M_{h} \frac{v_{W}^{2}}{\Lambda^{2}} \beta_{\chi}^{3}+\frac{\tilde{g}_{\chi}^{2}}{8 \pi} M_{h} \frac{v_{W}^{2}}{\Lambda^{2}} \beta_{\chi}, \\
\Gamma_{\chi \chi}^{V} & =\frac{g_{\chi}^{2}}{128 \pi} \frac{M_{h}^{3} v_{W}^{2}}{m_{\chi}^{4}} \beta_{\chi}\left(1-r_{\chi}+\frac{3}{4} r_{\chi}^{2}\right),
\end{aligned}
$$

where $r_{\chi}=4 m_{\chi}^{2} / M_{h}^{2}$ and $\beta_{\chi}=\sqrt{1-r_{\chi}}$.

The current best limits on the invisible branching fraction of the SM Higgs are obtained from $Z h$ production. The CMS collaboration gives a 95\% CL limit of $\operatorname{Br}(h \rightarrow$ inv $)<0.58$ for $M_{h}=125 \mathrm{GeV}$ [175] and ATLAS finds $\operatorname{Br}(h \rightarrow$ inv $)<0.75$ for $M_{h}=125.5$

$\mathrm{GeV}$ [176]. Note that the increased light-quark Yukawa couplings, at their presently allowed values, do not appreciably change the Higgs production cross section [159]. Their main effect is to increase the total decay width of the Higgs and thus reduce the 

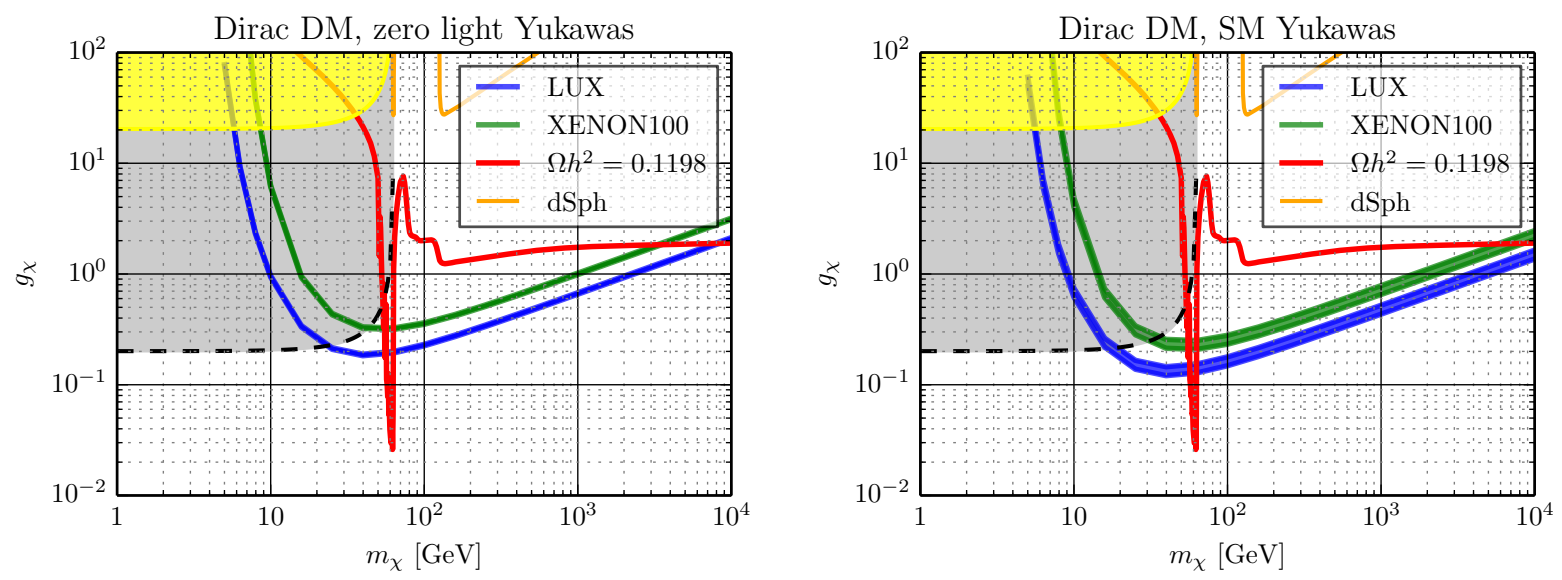

Figure 4.6: Bounds on the Higgs-portal coupling for Dirac DM, assuming $\Lambda=1 \mathrm{TeV}$ and vanishing (left) and SM (right) Yukawa couplings to $u, d, s$ quarks. The color coding is the same as in Fig. 4.2.

branching ratios to the other decay modes:

$$
\operatorname{Br}(h \rightarrow \chi \bar{\chi})=\frac{\Gamma(h \rightarrow \chi \bar{\chi})}{\Gamma(h \rightarrow \chi \bar{\chi})+\Gamma_{h}^{\text {tot }} \times\left[1+\sum_{q}\left(\kappa_{q}^{2}-1\right) \operatorname{Br}_{\mathrm{SM}}(h \rightarrow q \bar{q})\right]}
$$

In Figs. 4.2 to 4.9 we denote the bound on $g_{\chi}$ corresponding to the ATLAS upper limit on $\operatorname{Br}(h \rightarrow$ inv $)$ with a dashed black line and grey out the excluded region in the $g_{\chi}$ vs. $m_{\chi}$ plane. We see that the light DM Higgs portal, $m_{\chi} \lesssim m_{h} / 2$ is excluded by the Higgs invisible decay width.

Vector boson fusion, gluon fusion and $t \bar{t} H$ production, with the off-shell Higgs going to two DM particles, can provide some limited sensitivity to DM masses above $m_{h} / 2$. A combination of the searches in the three channels at a $100 \mathrm{TeV}$ collider could exclude the scalar thermal relic DM Higgs portal for DM masses in parts of the $m_{h} / 2 \lesssim$ $m_{\chi} \lesssim m_{W}$ interval at 95\% C.L. [156] (these result receives only a negligible correction if light quark Yukawa couplings are enhanced). For $m_{\chi}<m_{h} / 2$ the invisible Higgs decay width is, however, always the most constraining [177]. 


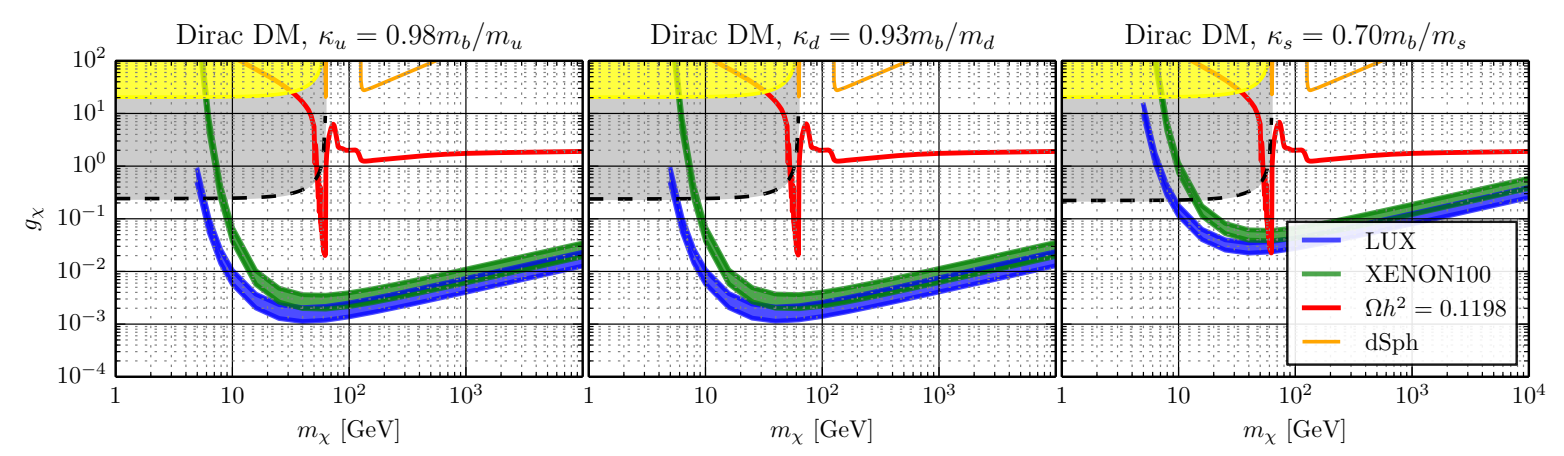

Figure 4.7: Bounds on the Higgs-portal coupling for Dirac DM, assuming $\Lambda=1 \mathrm{TeV}$ and maximal allowed values for the Yukawa couplings to the $u, d, s$ quarks (left to right), keeping all the other couplings to their SM values. The color coding is the same as in Fig. 4.2.

\subsubsection{Indirect detection}

In indirect signals of DM annihilation, the effect of changing the light-quark Yukawa couplings within the presently experimentally allowed ranges leads to at most $\mathcal{O}(1)$ effects. Further, the effect is present only for DM masses below the $W$ threshold where the dominant annihilation channel is into the $b \bar{b}$ final state. For example, Fig. 4.10 shows the recast of the Fermi-LAT bound from dwarf spheroidals [130] for scalar DM, following the procedure outlined below.

Photon flux measurements with $\gamma$-ray telescopes can put a strong bound on the annihilation cross-section of the DM. The strongest bound in the DM mass range of interest has been recently released by the Fermi-LAT collaboration [130] based on Pass 8 observation data of the Milky Way dwarf spheroidal satellite galaxies (dSphs). There is also a recent analysis based on the Dark Energy Survey (DES) dSph candidates using the Fermi-LAT data [178]. While this bound is competitive with the one from the known dSphs, it is still weaker on its own. One could also consider the bounds from the isotropic gamma ray background (IGRB) [132]. In our analysis, we recast the FermiLAT bound on the $b \bar{b}$ final state using a simple re-weighting procedure of the photon 

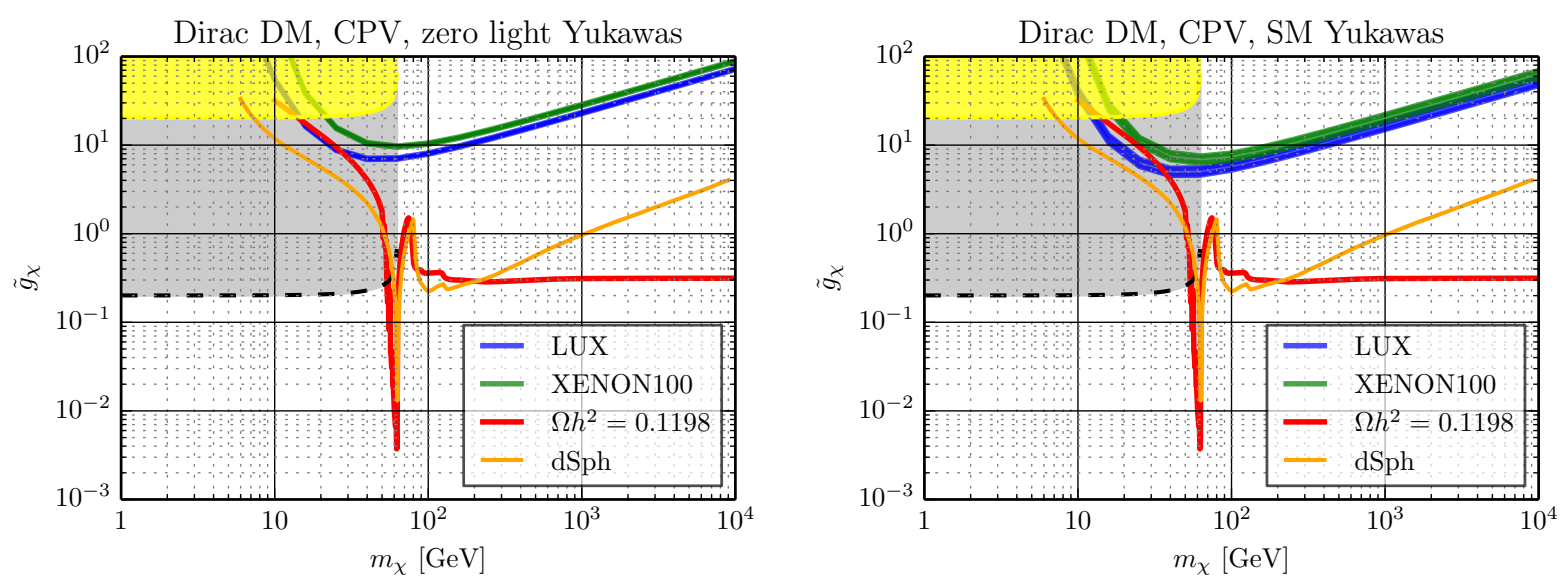

Figure 4.8: Bound on the pseudoscalar Higgs-portal coupling for Dirac DM, assuming $\Lambda=1 \mathrm{TeV}$ and vanishing (left) and SM (right) Yukawa couplings to $u, d, s$ quarks. The color coding is the same as in Fig. 4.2.

spectra which will be discussed below.

The observed differential photon flux from the annihilation of dark matter is given by

$$
\frac{d \Phi}{d E_{\gamma} d \Omega}=\frac{1}{4 \pi} \frac{1}{2 m_{\chi}^{2}} J\left[\sum_{f}\langle\sigma v\rangle_{f} \frac{d N_{\gamma}^{f}}{d E}\right],
$$

where $J$ is an astrophysical factor which depends on the distance to the source and the dark matter density profile. The factor in the brackets is the one most interesting for our purposes. It depends on the velocity-averaged cross-section and photon spectrum per DM annihilation.

The Fermi-LAT analysis gives bounds for the different final states separately while we have an admixture of final states. In order to recast the bound, we rely on the observation that for heavy DM the photon spectra from DM annihilation into quarks, gauge bosons, and the Higgs boson all peak at approximately the same photon energy and have approximately the same shape. Therefore, to extract the bound on the DM 


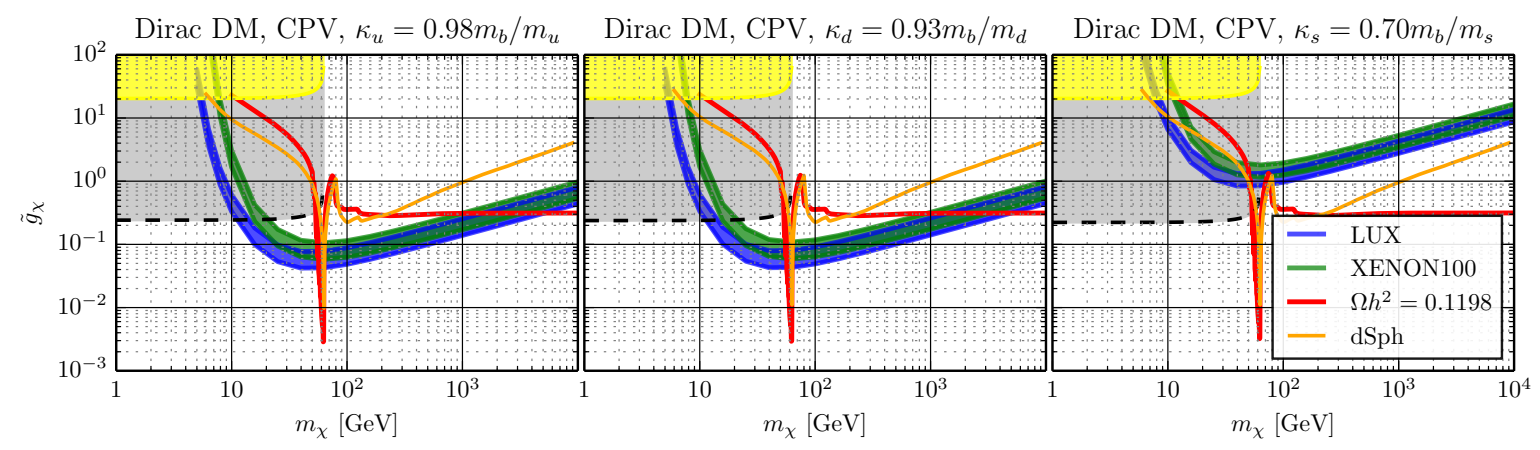

Figure 4.9: Bounds on the pseudoscalar Higgs-portal coupling for Dirac DM, assuming $\Lambda=1 \mathrm{TeV}$ and maximal allowed values for the Yukawa couplings to the $u, d, s$ quarks (left to right), keeping all the other couplings to their SM values. The color coding is the same as in Fig. 4.2.

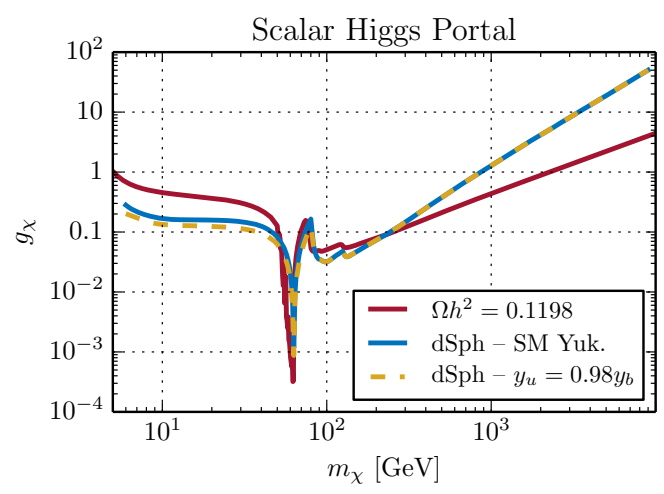

Figure 4.10: The effect of large light-quark Yukawa couplings on the indirect detection bounds from Fermi-LAT observations of Milky Way dwarf spheroidal satellite galaxies [130].

Higgs portal coupling $g_{\chi}$, it is sufficient to find the zeros of the polynomial

$$
f\left(g_{\chi}\right)=\sum_{f}\langle\sigma v\rangle_{f}\left(\frac{d N_{\gamma}^{f}}{d E}\right)^{\mathrm{PEAK}}-\langle\sigma v\rangle_{b}^{\mathrm{FERMI}}\left(\frac{d N_{\gamma}^{b}}{d E}\right)^{\mathrm{PEAK}}
$$

where in the last term, $\langle\sigma v\rangle_{b}^{\mathrm{FERMI}}$ is the bound from the Fermi-LAT analysis on the velocity-averaged cross-section. The photon spectra were obtained from the interpolation tables provided in the PPPC4DMID package [179]. In all cases except for the $h h$ final 
state, $f\left(g_{\chi}\right)$ has only one zero up to a sign ambiguity. For the $h h$ final state, however, the zero of $f\left(g_{\chi}\right)$ closest to the $g_{\chi}$ corresponding to $\chi \bar{\chi} \rightarrow b \bar{b}$ is the one used to rescale the Fermi-LAT bound on $g_{\chi}$ as a function of $m_{\chi}$. The resulting bounds are shown in Figs. 4.2 to 4.9 .

\subsubsection{Direct detection}

We have seen so far that most DM observables exhibit only a weak dependence on the light-quark Yukawas. This is not the case for the direct DM detection. In fact, modifying the light-quark Yukawa couplings can significantly change the predictions for DM - nucleus scattering cross sections.

The differential cross section for spin-independent DM scattering on a nucleus is given by

$$
\frac{d \sigma}{d E_{R}}=\frac{m_{A}}{\mu_{\chi A}^{2} v_{\mathrm{rel}}^{2}} \frac{\overline{|\mathcal{M}|^{2}}}{32 \pi s},
$$

where $E_{R}$ is the nuclear recoil energy, $m_{A}$ is the mass of the nucleus, $\mu_{\chi A} \equiv m_{\chi} m_{A} /\left(m_{\chi}+\right.$ $\left.m_{A}\right)$ is the reduced mass of the DM - nucleus system, $s=\left(m_{\chi}+m_{A}\right)^{2}$ is the centerof-mass energy, $v_{\text {rel }}$ is the DM velocity in the detector rest frame, and $\overline{|\mathcal{M}|^{2}}$ is the spinaveraged squared matrix element.

The matrix element $\mathcal{M}$ depends on the effective Higgs couplings to the nucleus. Since the momentum exchanges in DM scattering on nuclei are much smaller than the Higgs mass, we can calculate $\overline{|\mathcal{M}|^{2}}$ by first integrating out the Higgs and the heavy quarks $(t, b, c)$. This gives an EFT with light quarks and gluons interacting with DM 
through local operators, described by the effective Lagrangians

$$
\begin{aligned}
\mathcal{L}_{S} & =\frac{g_{\chi} v_{W}}{m_{h}^{2}}\left(\chi^{\dagger} \chi\right) \mathcal{S}_{q}, \\
\mathcal{L}_{F} & =\frac{1}{\Lambda} \frac{g_{\chi} v_{W}}{m_{h}^{2}}(\bar{\chi} \chi) \mathcal{S}_{q}+\frac{1}{\Lambda} \frac{\tilde{g}_{\chi} v_{W}}{m_{h}^{2}}\left(\bar{\chi} i \gamma_{5} \chi\right) \mathcal{S}_{q} . \\
\mathcal{L}_{V} & =\frac{g_{\chi} v_{W}}{2 m_{h}^{2}}\left(\chi_{\mu} \chi^{\mu}\right) \mathcal{S}_{q},
\end{aligned}
$$

for scalar, fermion, and vector DM, respectively. The scalar current is the same in all three cases:

$$
\mathcal{S}_{q}=\sum_{q} \kappa_{q} \frac{m_{q}}{v_{W}} \bar{q} q-\mathcal{C}_{g} \frac{\alpha_{s}}{12 \pi v_{W}} G_{\mu \nu}^{a} G^{a \mu \nu}+\sum_{q} i \tilde{\kappa}_{q} \frac{m_{q}}{v_{W}} \bar{q} \gamma_{5} q-\tilde{\mathcal{C}}_{g} \frac{\alpha_{s}}{8 \pi v_{W}} G_{\mu \nu}^{a} \widetilde{G}^{a \mu \nu}
$$

Here, the last two terms arise from $C P$-violating Higgs couplings. The sums are over the light quarks $q=u, d, s$. The heavy quarks are integrated out and contribute only via the gluonic terms in the current. For the two corresponding dimensionless Wilson coefficients we have

$$
\mathcal{C}_{g}=\kappa_{g}^{\mathrm{NP}}+\kappa_{t}+\kappa_{b}+\kappa_{c}, \quad \tilde{\mathcal{C}}_{g}=\tilde{\kappa}_{g}^{\mathrm{NP}}+\tilde{\kappa}_{t}+\tilde{\kappa}_{b}+\tilde{\kappa}_{c},
$$

where the first contribution is from tree-level matching, and the remaining from oneloop matching, working in the limit of heavy quarks. This is well justified for top and bottom quarks. For scattering on heavy nuclei, e.g., on Xe or W, the maximal momentum exchanges for $\mathrm{DM}$ with mass above approximately $1 \mathrm{TeV}$ may, however, start to become comparable to the charm-quark mass. We neglect these effects, while they may need to be included in the future if such heavy DM is discovered.

$C P$-violating Higgs couplings to light quarks lead to spin-dependent interactions of DM with the target nuclei. The corresponding scattering rates are suppressed relative to the spin-independent interaction rates from $C P$-conserving Higgs couplings. We 
will therefore neglect the $C P$-violating interactions in our numerical analysis of direct detection scattering rates; i.e., we will set $\tilde{\kappa}_{q}=0, \tilde{\mathcal{C}}_{q}=0$ from now on.

The nucleon matrix elements of the remaining terms in the scalar current $\mathcal{S}_{q}$ are conventionally parametrized by (see, e.g., [87]),

$$
\begin{aligned}
\left\langle N\left|m_{q} \bar{q} q\right| N\right\rangle & =m_{N} f_{T q}^{(N)}, \\
\left\langle N\left|\frac{\alpha_{s}}{12 \pi} G_{\mu \nu} G^{\mu \nu}\right| N\right\rangle & =-\frac{2}{27} m_{N} f_{T G}^{(N)} .
\end{aligned}
$$

In the heavy-quark limit for $t, b, c$ the trace anomaly equation leads to the relation [87, 180]

$$
f_{T G}^{(N)}=1-\sum_{q=u, d, s} f_{T q}^{(N)} .
$$

We can also define the effective Higgs coupling to nucleon as the expectation value of the scalar current,

$$
f_{\mathcal{S}}^{(N)} \equiv\left\langle N\left|\mathcal{S}_{q}\right| N\right\rangle=\frac{m_{N}}{v_{W}}\left[\frac{2}{27} \mathcal{C}_{g}+\sum_{q}\left(\kappa_{q}-\frac{2}{27} \mathcal{C}_{g}\right) f_{T q}^{(N)}\right]
$$

The exclusion curves from LUX [120] and Xenon100 [181], assuming a local DM density of $0.3 \mathrm{GeV} / \mathrm{cm}^{3}$, are shown in Figs. 4.2 to 4.9 as blue and red bands, respectively. The width of the exclusion curves represents the uncertainties in the hadronic matrix elements of the light-quark scalar currents. For the $s$ quark we use the lattice determination $f_{T s}^{(N)}=0.043 \pm 0.011$ [123]. The matrix elements for $u$ and $d$ quarks can be related to the $\sigma_{\pi N}$ term. A Baryon Chiral Perturbation Theory (B $\chi \mathrm{PT}$ ) analysis of the $\pi N$ scattering data gives $\sigma_{\pi N}=59(7) \mathrm{MeV}$ [124]. This is in agreement with $\mathrm{B} \chi \mathrm{PT}$ fit to world lattice $N_{f}=2+1$ QCD data, which gives $\sigma_{\pi N}=52(3)(8) \mathrm{MeV}$ [125]. Including both $\Delta(1232)$ and finite spacing parametrization in the fit shifts the central value to $\sigma_{\pi N}=44 \mathrm{MeV}$. To be conservative we use $\sigma_{\pi N}=(50 \pm 15) \mathrm{MeV}$, which 
gives $f_{T u}^{(p)}=(1.8 \pm 0.5) \times 10^{-2}, f_{T d}^{(p)}=(3.4 \pm 1.1) \times 10^{-2}, f_{T u}^{(n)}=(1.6 \pm 0.5) \times 10^{-2}$, $f_{T d}^{(n)}=(3.8 \pm 1.1) \times 10^{-2}$, using the expressions in $[126,127]$. For the effective Higgs coupling to nucleons this gives

$$
\begin{aligned}
f_{\mathcal{S}}^{(p)}=\frac{m_{W}}{v_{W}}[ & (1.8 \pm 0.5) \kappa_{u}+(3.4 \pm 1.1) \kappa_{d}+(4.3 \pm 1.1) \kappa_{s} \\
& \left.+(6.70 \pm 0.12)\left(\kappa_{c}+\kappa_{b}+\kappa_{t}+\kappa_{g}^{\mathrm{NP}}\right)\right] \times 10^{-2} \\
f_{\mathcal{S}}^{(n)}=\frac{m_{W}}{v_{W}}[ & (1.6 \pm 0.5) \kappa_{u}+(3.8 \pm 1.1) \kappa_{d}+(4.3 \pm 1.1) \kappa_{s} \\
& \left.+(6.69 \pm 0.12)\left(\kappa_{c}+\kappa_{b}+\kappa_{t}+\kappa_{g}^{\mathrm{NP}}\right)\right] \times 10^{-2} .
\end{aligned}
$$

We use the results in [182] to relate the nuclear matrix elements to actual scattering rates on nuclei via nuclear form factors.

We show the direct detection exclusion limits for SM $\left(\kappa_{u, d, s}=1\right)$ or vanishing $\left(\kappa_{u, d, s}=0\right)$ light-quark Yukawa couplings in the right and left panels in Figs. 4.2, 4.4, 4.6, and 4.8 , respectively. The exclusion limits are approximately two times weaker in the latter case; the constraint does not vanish because, for small values of the light-quark Yukawas, the scattering cross section is dominated by the gluon part of the scalar current, Eq. (4.25). When the light-quark Yukawas are taken to be at the upper limit of their experimentally allowed range, i.e. comparable to the SM bottom Yukawa, the direct detection bounds on $g_{\chi}$ become significantly stronger, by a factor of about $m_{b} / m_{q}$ (Figs. 4.3, 4.5, 4.7, and 4.9).

It is interesting to note that, because of the dominance of the gluon contribution, for small light-quark Yukawas the theory uncertainty in the exclusion bands is significantly smaller than if the light Yukawa couplings are allowed to saturate the present experimental bounds. (The nuclear matrix element of the effective gluon term has smaller relative uncertainties than the corresponding matrix elements of $m_{q} \bar{q} q$ since $f_{T G}^{(N)} \gg f_{T q}^{(N)}$.) 

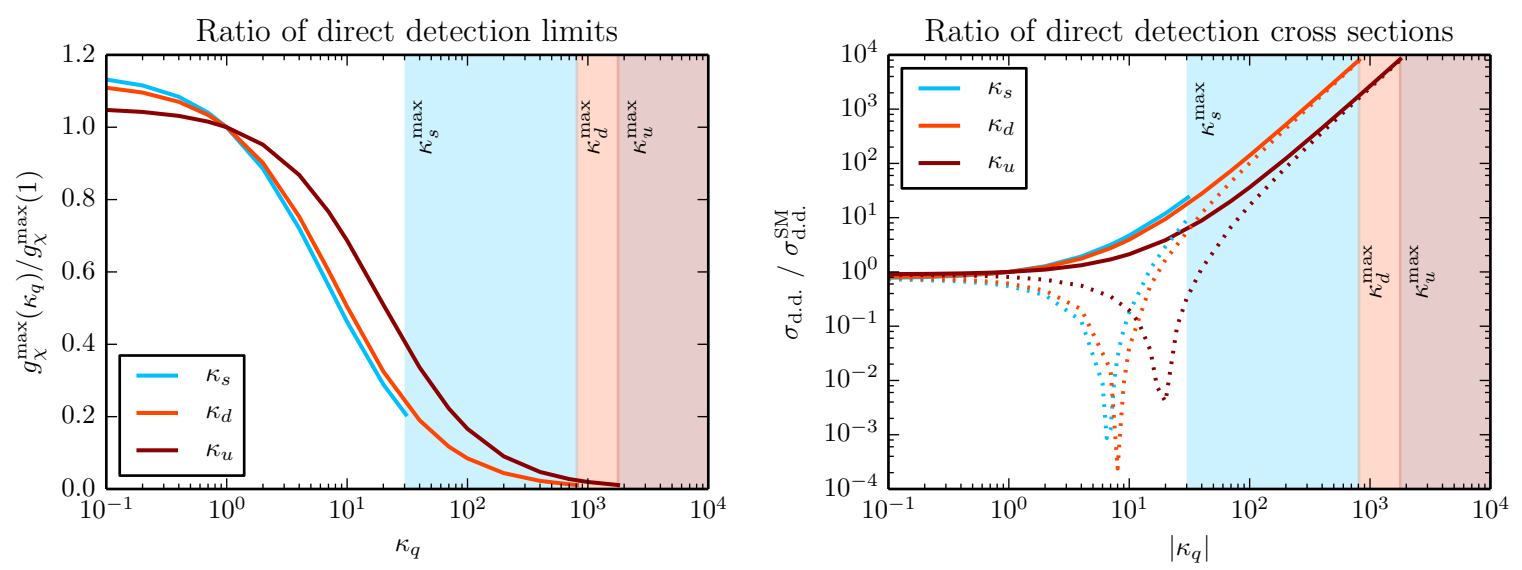

Figure 4.11: Left: The ratio of direct detection bounds on $g_{\chi}$ from Xenon target varying $\kappa_{u}$ (dark red), $\kappa_{d}$ (light red), or $\kappa_{s}$ (blue), and the bound on $g_{\chi}$ assuming SM Higgs Yukawa couplings. The LHC upper bounds on $\kappa_{i}$ are denoted by vertical dashed lines with shaded regions excluded. Right: the ratio of predicted scattering cross sections. The dotted lines correspond to negative values of $\kappa_{q}$.

For $m_{\chi}$ smaller than a few $\mathrm{TeV}$, the $\mathrm{DM}$ direct detection bounds are compatible with the thermal relic Higgs-portal DM only if light quark Yukawas are well below the present experimental bounds (the exception is a pseudoscalar fermion DM with enhanced strange Yukawa, where the bound is $m_{\chi} \gtrsim m_{h} / 2$, see Fig. 4.9). This means that if thermal relic DM is discovered, it would immediately place an upper bound on $\kappa_{u}, \kappa_{d}, \kappa_{s}$, assuming Higgs-portal mediation (unless in the case of fermion DM that has purely pseudoscalar couplings). We comment in more detail on that observation in Section 4.4.

Since the DM - nucleus scattering cross section is the only DM observable that exhibits a rather pronounced dependence on the values of the light-quark Yukawas, we study this dependence in more detail.

In Fig. 4.11 (left) we show how the direct detection bounds on $g_{\chi}$ are affected by 
changes in the values of the light-quark Yukawas. We plot the ratio

$$
\xi_{g_{\chi}}=\frac{g_{\chi}^{\max }\left(\kappa_{q}\right)}{g_{\chi}^{\max }(1)}
$$

where $g_{\chi}^{\max }\left(\kappa_{q}\right)$ is the upper bound on $g_{\chi}$ obtained from direct detection experiments for a given value of $\kappa_{q}$, with $q=u, d, s$. Hence, $g_{\chi}^{\max }(1)$ is the bound obtained assuming SM Yukawa couplings. Its value depends on $m_{\chi}$, on whether DM is a scalar, fermion, or vector, and on the experiment that measured the bounds. Similarly, also $g_{\chi}^{\max }\left(\kappa_{q}\right)$ depends on $m_{\chi}$, the spin of DM, and the experiment; however, all these dependences cancel in the ratio $\xi_{g_{\chi}}$. The ratio $\xi_{g_{\chi}}$ thus only depends on $\kappa_{q}$ and on which target material was used to derive the direct detection bounds. In Fig. 4.11 we show $\xi_{g_{\chi}}$ for a Xenon target, varying in turn $\kappa_{u}$ (dark red line), $\kappa_{d}$ (light red) and $\kappa_{s}$ (blue), while keeping all other parameters fixed to their SM values. We set the hadronic matrix element $f_{T q}^{(N)}$ to their present central values, anticipating that in the future their uncertainties will be further reduced. In Fig. 4.11 (right) we show a closely related quantity - the ratio of the scattering cross sections with varied $\kappa_{u, d, s}$ and the scattering cross section with SM Yukawa couplings, $\sigma_{\text {d.d. }} / \sigma_{\text {d.d. }}^{\mathrm{SM}}$.

Fig. 4.11 illustrates clearly that the difference between the bounds where one of the light quark Yukawa couplings is taken to be small or vanish completely, and the bounds where all the couplings are SM-like, is very small, $\mathcal{O}(10 \%)$. Saturating the present experimental bounds on $\kappa_{u}$ or $\kappa_{d}$, the allowed value of $g_{\chi}$ could lie two orders of magnitude below what one obtains for the case of SM Yukawa couplings. Such large values for the light-quark Yukawas are not very likely to be realized in a concrete model, as we will discuss in the next section. However, it is very interesting to observe that even a moderate increase of the values of the light-quark Yukawa couplings to only a few times their SM values can have a significant effect on the direct detection bounds, enhancing the scattering cross sections by up to a factor of ten. 
Finally, in the right panel of Fig. 4.11 we show the cross section ratios for negative values of the $\kappa_{q}$ (dotted lines). We see that the interference of the light-quark contributions with the effective gluon interaction can, in principle, lead to a strong reduction of the scattering cross section.

\subsection{Changes to Yukawa couplings in new physics models}

So far we allowed the Yukawa couplings of the Higgs to quarks to have arbitrary values, only restricting them to lie within the bounds obtained from global fits to LHC data. For simplicity, we also neglected flavor violation and $C P$ violation when discussing their impact on the DM interactions.

Of course, changes of the Yukawa couplings by several orders of magnitude, as allowed by current experimental constraints on the light-quark Yukawas, are not very likely to be realized in a complete model, and might require significant fine tuning of the corresponding quark masses. In this section we investigate how large the deviations from the SM Yukawa interactions can be in popular models of NP with viable flavor structures.

Tables 4.1 and 4.2 summarize the predictions for the effective Yukawa couplings in the Standard Model (SM), in multi-Higgs-doublet models (MHDM) with natural flavor conservation (NFC) $[183,184]$, in the MSSM at tree level, the Giudice-Lebedev model of quark masses (GL) [185], in NP models with minimal flavor violation (MFV) [64], in Randall-Sundrum models (RS) [186], and in models with a composite Higgs, realized as a pseudo-Nambu-Goldstone boson (pNGB) [187-190]. For completeness, we include both the flavor-conserving and flavor-violating Yukawa interactions, and allow for $C P$ violation. The Higgs couplings to quarks are thus described by

$$
\mathcal{L}_{\text {eff, }, \mathrm{q}}=-\kappa_{q} \frac{m_{q}}{v_{W}} \bar{q} q h-i \tilde{\kappa}_{q} \frac{m_{q}}{v_{W}} \bar{q} \gamma_{5} q h-\left[\left(\kappa_{q q^{\prime}}+i \tilde{\kappa}_{q q^{\prime}}\right) \bar{q}_{L} q_{R}^{\prime} h+\text { h.c. }\right]
$$




\begin{tabular}{lcccc}
\hline \hline Model & $\kappa_{t}$ & $\kappa_{c(u)} / \kappa_{t}$ & $\tilde{\kappa}_{t} / \kappa_{t}$ & $\tilde{\kappa}_{c(u)} / \kappa_{t}$ \\
\hline SM & 1 & 1 & 0 & 0 \\
NFC & $V_{h u} v_{W} / v_{u}$ & 1 & 0 & 0 \\
MSSM & $\cos \alpha / \sin \beta$ & 1 & 0 & 0 \\
GL & $1+\mathcal{O}\left(\epsilon^{2}\right)$ & $\simeq 3(7)$ & $\mathcal{O}\left(\epsilon^{2}\right)$ & $\mathcal{O}\left(\kappa_{c(u)}\right)$ \\
GL2 & $\cos \alpha / \sin \beta$ & $\simeq 3(7)$ & $\mathcal{O}\left(\epsilon^{2}\right)$ & $\mathcal{O}\left(\kappa_{c(u)}\right)$ \\
MFV & $1+\frac{\operatorname{Re}\left(a_{u} v_{W}^{2}+2 b_{u} m_{t}^{2}\right)}{\Lambda^{2}}$ & $1-\frac{2 \operatorname{Re}\left(b_{u}\right) m_{t}^{2}}{\Lambda^{2}}$ & $\frac{\Im\left(a_{u} v_{W}^{2}+2 b_{u} m_{t}^{2}\right)}{\Lambda^{2}}$ & $\frac{\Im\left(a_{u} v_{W}^{2}\right)}{\Lambda^{2}}$ \\
RS & $1-\mathcal{O}\left(\frac{v_{W}^{2}}{m_{K K}^{2}} \bar{Y}^{2}\right)$ & $1+\mathcal{O}\left(\frac{v_{W}^{2}}{m_{K K}^{2}} \bar{Y}^{2}\right)$ & $1+\mathcal{O}\left(\frac{v_{W}^{2}}{m_{K K}^{2}} \bar{Y}^{2}\right)$ & $1+\mathcal{O}\left(\frac{v_{W}^{2}}{m_{K K}^{2}} \bar{Y}^{2}\right)$ \\
pNGB & $1+\mathcal{O}\left(\frac{v_{W}^{2}}{f^{2}}\right)+\mathcal{O}\left(y_{*}^{2} \lambda^{2} \frac{v_{W}^{2}}{M_{*}^{2}}\right)$ & $1+\mathcal{O}\left(y_{*}^{2} \lambda^{2} \frac{v_{W}^{2}}{M_{*}^{2}}\right)$ & $\mathcal{O}\left(y_{*}^{2} \lambda^{2} \frac{v_{W}^{2}}{M_{*}^{2}}\right)$ & $\mathcal{O}\left(y_{*}^{2} \lambda^{2} \frac{v_{W}^{2}}{M_{*}^{2}}\right)$ \\
\hline \hline
\end{tabular}

Table 4.1: Predictions for the flavor diagonal up-type Yukawa couplings in a number of new physics models (see text for details).

where a sum over the SM quark fields is understood. The first two terms are flavor diagonal, with the first term $C P$ conserving and the second term $C P$ violating, and coincide with the definitions in eqs. (4.3) and (4.5), respectively. The terms in square brackets are flavor violating, with the real (imaginary) part of the coefficient $C P$ conserving (violating). In the SM we have $\kappa_{q}=1$, while $\tilde{\kappa}_{q}=\kappa_{q q^{\prime}}=\tilde{\kappa}_{q q^{\prime}}=0$. The flavor-violating couplings in the above set of NP models are collected in Tables 4.3 and 4.4. These tables complement the analyses in [191-193] (see also [194], where implications of a negative top-quark Yukawa were explored, and [195] for an indirect bound on the down-quark Yukawa in alignment models).

\subsubsection{Dimension-Six Operators with Minimal Flavor Violation}

We start our discussion by considering dimension-six operators arising from integrating out NP at a high scale $\Lambda$. In addition, we assume that the flavor breaking in the NP sector is only due to the SM Yukawas, i.e. that NP satisfies the Minimal Flavor Violation (MFV) hypothesis [63,64,66,67,98-100]. Integrating out the new physics states 


\begin{tabular}{lcccc}
\hline \hline Model & $\kappa_{b}$ & $\kappa_{s(d)} / \kappa_{b}$ & $\tilde{\kappa}_{b} / \kappa_{b}$ & $\tilde{\kappa}_{s(d)} / \kappa_{b}$ \\
\hline SM & 1 & 1 & 0 & 0 \\
NFC & $V_{h d} v_{W} / v_{d}$ & 1 & 0 & 0 \\
MSSM & $-\sin \alpha / \cos \beta$ & 1 & 0 & 0 \\
GL & $\simeq 3$ & $\simeq 5 / 3(7 / 3)$ & $\mathcal{O}(1)$ & $\mathcal{O}\left(\kappa_{s(d)} / \kappa_{b}\right)$ \\
GL2 & $-\sin \alpha / \cos \beta$ & $\simeq 3(5)$ & $\mathcal{O}\left(\epsilon^{2}\right)$ & $\mathcal{O}\left(\kappa_{s(d)} / \kappa_{b}\right)$ \\
MFV & $1+\frac{\operatorname{Re}\left(a_{d} v_{W}^{2}+2 c_{d} m_{t}^{2}\right)}{\Lambda^{2}}$ & $1-\frac{2 \operatorname{Re}\left(c_{d}\right) m_{t}^{2}}{\Lambda^{2}}$ & $\frac{\Im\left(a_{d} v_{W}^{2}+2 c_{d} m_{t}^{2}\right)}{\Lambda^{2}}$ & $\frac{\Im\left(a_{d} v_{W}^{2}+2 c_{d}\left|V_{t s(t d)}\right|^{2} m_{t}^{2}\right)}{\Lambda^{2}}$ \\
RS & $1-\mathcal{O}\left(\frac{v_{W}^{2}}{m_{K K}^{2}} \bar{Y}^{2}\right)$ & $1+\mathcal{O}\left(\frac{v_{W}^{2}}{m_{K K}^{2}} \bar{Y}^{2}\right)$ & $1+\mathcal{O}\left(\frac{v_{W}^{2}}{m_{K K}^{2}} \bar{Y}^{2}\right)$ & $1+\mathcal{O}\left(\frac{v_{W}^{2}}{m_{K K}^{2}} \bar{Y}^{2}\right)$ \\
pNGB & $1+\mathcal{O}\left(\frac{v_{W}^{2}}{f^{2}}\right)+\mathcal{O}\left(y_{*}^{2} \lambda^{2} \frac{v_{W}^{2}}{M_{*}^{2}}\right)$ & $1+\mathcal{O}\left(y_{*}^{2} \lambda^{2} \frac{v_{W}^{2}}{M_{*}^{2}}\right)$ & $\mathcal{O}\left(y_{*}^{2} \lambda^{2} \frac{v_{W}^{2}}{M_{*}^{2}}\right)$ & $\mathcal{O}\left(y_{*}^{2} \lambda^{2} \frac{v_{W}^{2}}{M_{*}^{2}}\right)$ \\
\hline \hline
\end{tabular}

Table 4.2: Predictions for the flavor diagonal down-type Yukawa couplings in a number of new physics models (see text for details).

gives for the Higgs couplings to quarks

$$
\mathcal{L}_{\mathrm{EFT}}=Y_{u} \bar{Q}_{L} H^{c} u_{R}+Y_{d} \bar{Q}_{L} H d_{R}+\frac{Y_{u}^{\prime}}{\Lambda^{2}} \bar{Q}_{L} H^{c} u_{R}\left(H^{\dagger} H\right)+\frac{Y_{d}^{\prime}}{\Lambda^{2}} \bar{Q}_{L} H d_{R}\left(H^{\dagger} H\right)+\text { h.c. },
$$

where $\Lambda$ is the scale of new physics and $H^{c}=i \sigma_{2} H^{*}$. We identify the NP scales in the up- and down-quark sectors for simplicity. There are also modifications of quark kinetic terms through dimension-six derivative operators. These can be absorbed in (4.35) using equations of motion [196]. The quark mass matrices and Yukawa couplings after EWSB are thus

$$
M_{q}=\frac{v_{W}}{\sqrt{2}}\left(Y_{q}+Y_{q}^{\prime} \frac{v_{W}^{2}}{2 \Lambda^{2}}\right), \quad y_{q}=Y_{q}+3 Y_{q}^{\prime} \frac{v_{W}^{2}}{2 \Lambda^{2}}, \quad q=u, d
$$

Because $Y_{q}$ and $Y_{q^{\prime}}$ appear in two different combinations in $M_{q}$ and $y_{q}$, the two, in general, cannot be made diagonal in the same basis.

In MFV the coefficients of the dimension-six operators can be expanded in terms of 


\begin{tabular}{lccc}
\hline \hline Model & $\kappa_{c t(t c)} / \kappa_{t}$ & $\kappa_{u t(t u)} / \kappa_{t}$ & $\kappa_{u c(c u)} / \kappa_{t}$ \\
\hline GL \& GL2 & $\epsilon\left(\epsilon^{2}\right)$ & $\epsilon\left(\epsilon^{2}\right)$ & $\epsilon^{3}$ \\
MFV & $\frac{\operatorname{Re}\left(c_{u} m_{b}^{2} V_{c b}^{(*)}\right)}{\Lambda^{2}} \frac{\sqrt{2} m_{t(c)}}{v_{W}}$ & $\frac{\operatorname{Re}\left(c_{u} m_{b}^{2} V_{u b}^{(*)}\right)}{\Lambda^{2}} \frac{\sqrt{2} m_{t(u)}}{v_{W}}$ & $\frac{\operatorname{Re}\left(c_{u} m_{b}^{2} V_{u b(c b)} V_{c b(u b)}^{*}\right)}{\Lambda^{2}} \frac{\sqrt{2} m_{c(u)}}{v_{W}}$ \\
RS & $\sim \lambda^{(-) 2} \frac{m_{t(c)}}{v_{W}} \bar{Y}^{2} \frac{v_{W}^{2}}{m_{K K}^{2}}$ & $\sim \lambda^{(-) 3} \frac{m_{t(u)}}{v_{W}} \bar{Y}^{2} \frac{v_{W}^{2}}{m_{K K}^{2}}$ & $\sim \lambda^{(-) 1} \frac{m_{c(u)}}{v_{W}} \bar{Y}^{2} \frac{v_{W}^{2}}{m_{K K}^{2}}$ \\
pNGB & $\mathcal{O}\left(y_{*}^{2} \frac{m_{t}}{v_{W}} \frac{\lambda_{L(R), 2} \lambda_{L(R), 3} m_{W}^{2}}{M_{*}^{2}}\right)$ & $\mathcal{O}\left(y_{*}^{2} \frac{m_{t}}{v_{W}} \frac{\lambda_{L(R), 1} \lambda_{L(R), 3} m_{W}^{2}}{M_{*}^{2}}\right)$ & $\mathcal{O}\left(y_{*}^{2} \frac{m_{c}}{v_{W}} \frac{\lambda_{L(R), 1} \lambda_{L(R), 2} m_{W}^{2}}{M_{*}^{2}}\right)$ \\
\hline \hline
\end{tabular}

Table 4.3: Predictions for the flavor violating up-type Yukawa couplings in a number of new physics models (see text for details). In the SM, NFC and the tree-level MSSM the Higgs Yukawa couplings are flavor diagonal. The estimates of the $C P$-violating versions of the flavor-changing transitions, $\kappa_{i j} / \kappa_{t}$, are the same as the $C P$-conserving ones, apart from substituting " $\mathrm{Im}$ " for "Re" in the "MFV" row.

\begin{tabular}{lccc}
\hline \hline Model & $\kappa_{b s(s b)} / \kappa_{b}$ & $\kappa_{b d(d b)} / \kappa_{b}$ & $\kappa_{s d(d s)} / \kappa_{b}$ \\
\hline GL \& GL2 & $\epsilon^{3}\left(\epsilon^{2}\right)$ & $\epsilon^{2}$ & $\epsilon^{3}\left(\epsilon^{4}\right)$ \\
MFV & $\frac{\operatorname{Re}\left(c_{d} m_{t}^{2} V_{t s}^{(*)}\right)}{\Lambda^{2}} \frac{\sqrt{2} m_{s(b)}}{v_{W}}$ & $\frac{\operatorname{Re}\left(c_{d} m_{t}^{2} V_{t d}^{(*)}\right)}{\Lambda^{2}} \frac{\sqrt{2} m_{d(b)}}{v_{W}}$ & $\frac{\operatorname{Re}\left(c_{d} m_{t}^{2} V_{t s(t d)}^{*} V_{t d(t s)}\right)}{\Lambda^{2}} \frac{\sqrt{2} m_{s(d)}}{v_{W}}$ \\
RS & $\sim \lambda^{(-) 2} \frac{m_{b(s)}}{v_{W}} \bar{Y}^{2} \frac{v_{W}^{2}}{m_{K K}^{2}}$ & $\sim \lambda^{(-) 3} \frac{m_{b(d)}}{v_{W}} \bar{Y}^{2} \frac{v_{W}^{2}}{m_{K K}^{2}}$ & $\sim \lambda^{(-) 1} \frac{m_{s(d)}}{v_{W}} \bar{Y}^{2} \frac{v_{W}^{2}}{m_{K K}^{2}}$ \\
pNGB & $\mathcal{O}\left(y_{*}^{2} \frac{m_{b}}{v_{W}} \frac{\lambda_{L(R), 2} \lambda_{L(R), 3} m_{W}^{2}}{M_{*}^{2}}\right)$ & $\mathcal{O}\left(y_{*}^{2} \frac{m_{b}}{v_{W}} \frac{\lambda_{L(R), 1} \lambda_{L(R), 3} m_{W}^{2}}{M_{*}^{2}}\right)$ & $\mathcal{O}\left(y_{*}^{2} \frac{m_{s}}{v_{W}} \frac{\lambda_{L(R), 1} \lambda_{L(R), 2} m_{W}^{2}}{M_{*}^{2}}\right)$ \\
\hline \hline
\end{tabular}

Table 4.4: Predictions for the flavor violating down-type Yukawa couplings in a number of new physics models (see text for details). In SM, NFC and tree level MSSM the Higgs Yukawa couplings are flavor diagonal. The estimates of the $C P$-violating versions of the flavor-changing transitions, $\kappa_{i j} / \kappa_{b}$, are the same as the $C P$-conserving ones, apart from substituting "Im" for "Re" in the "MFV" row.

$Y_{u, d}$

$$
\begin{aligned}
& Y_{u}^{\prime}=a_{u} Y_{u}+b_{u} Y_{u} Y_{u}^{\dagger} Y_{u}+c_{u} Y_{d} Y_{d}^{\dagger} Y_{u}+\cdots \\
& Y_{d}^{\prime}=a_{d} Y_{d}+b_{d} Y_{d} Y_{d}^{\dagger} Y_{d}+c_{d} Y_{u} Y_{u}^{\dagger} Y_{d}+\cdots
\end{aligned}
$$

with $a_{q}, b_{q}, c_{q} \sim \mathcal{O}(1)$. Working to first order in dimension-six operator insertions we can thus write for the Yukawa couplings, in the mass eigenbases for up and down 
quarks respectively,

$$
\begin{aligned}
& y_{u}=\left[1+\frac{v_{W}^{2}}{\Lambda^{2}}\left(a_{u}+b_{u}\left(y_{\mathrm{SM}}^{u}\right)^{2}+c_{u} V\left(y_{\mathrm{SM}}^{d}\right)^{2} V^{\dagger}+\cdots\right)\right] y_{\mathrm{SM}}^{u}, \\
& y_{d}=\left[1+\frac{v_{W}^{2}}{\Lambda^{2}}\left(a_{d}+b_{d}\left(y_{\mathrm{SM}}^{d}\right)^{2}+c_{d} V^{\dagger}\left(y_{\mathrm{SM}}^{u}\right)^{2} V+\cdots\right)\right] y_{\mathrm{SM}}^{d} .
\end{aligned}
$$

Here $y_{\mathrm{SM}}^{u, d}$ are the diagonal matrices of the SM Yukawa couplings, while $V$ is the CabibboKobayashi-Maskawa (CKM) matrix. In general, the coefficients $a_{q}, b_{q}, c_{q}$ are complex so that $C P$-violating Higgs couplings arise at $\mathcal{O}\left(v_{W}^{2} / \Lambda^{2}\right)$. Flavor-violating Higgs couplings arise first from the terms proportional to $c_{u, d}$ in the Yukawa expansion and are thus suppressed by the corresponding CKM matrix elements. In Tables 4.1-4.4 we collect the values for flavor-conserving and flavor-violating Yukawa couplings in the "MFV" row, assuming that all the coefficients $a_{q}, b_{q}, c_{q}$ are $\mathcal{O}(1)$, and show only the numerically leading non-SM contributions. In the expressions we also set $V_{t b}$ to unity.

The corrections to DM phenomenology are dominated by changes of the thirdgeneration Yukawa couplings. The MFV corrections to light-quark Yukawa couplings

are all either additionally CKM suppressed or involve extra insertions of light-quark masses. Hence the theory error in Higgs-portal DM phenomenology due to Yukawa coupling uncertainties will be small in MFV models of NP once the Higgs couplings to top and bottom quarks are well measured.

\subsubsection{Multi-Higgs-doublet model with natural flavor conservation}

In MHDMs there are no tree-level FCNCs if natural flavor conservation is assumed [183, 184]. Under this assumption we can choose a Higgs doublet basis in which only one doublet, $H_{u}$, couples to the up-type quarks, and only one Higgs doublet, $H_{d}$, couples to the down-type quarks ${ }^{2}$. After EWSB the two doublets obtain the vevs $v_{u}$ and $v_{d}$, respectively. On the other hand, the vevs of all Higgs doublets contribute to the $W$

\footnotetext{
${ }^{2}$ Note that $H_{u}=H_{d}$ is included as a special case.
} 
and $Z$ masses. They satisfy the sum rule $v_{W}^{2}=\sum_{i} v_{i}^{2}$, where the sum is over all Higgs doublets.

The neutral scalar components of $H_{i}$ are $\left(v_{i}+h_{i}\right) / \sqrt{2}$, where the dynamical fields $h_{i}$ are a linear combination of the neutral Higgs mass eigenstates (and include $h_{u}$ and $\left.h_{d}\right)$. We thus have $h_{i}=V_{h i} h+\ldots$, where $V_{h i}$ are elements of the unitary matrix $V$ that diagonalizes the neutral-Higgs mass terms and we only write down the contribution of the lightest Higgs, $h$. Under the assumptions above, the mass and Yukawa terms can be diagonalized in the same basis, so that there is no flavor violation and no $C P$ violation in the Yukawa interactions:

$$
\kappa_{q q^{\prime}}=\tilde{\kappa}_{q q^{\prime}}=0, \quad \tilde{\kappa}_{q}=0 .
$$

We obtain a universal shift in all up-quark Yukawa couplings, and a different universal shift in all down-quark Yukawa couplings, given by

$$
\kappa_{u}=\kappa_{c}=\kappa_{t}=V_{h u} \frac{v_{W}}{v_{u}}, \quad \kappa_{d}=\kappa_{s}=\kappa_{b}=V_{h d} \frac{v_{W}}{v_{d}} .
$$

Since the shifts are universal over generations, the precise measurements of the Higgs couplings to top and bottom quarks will also determine the Higgs couplings to light quarks. Both $\kappa_{t}$ and $\kappa_{b}$ are expected to be known with $\mathcal{O}(5 \%)$ precision after the end of the high-luminosity LHC run $[197,198]$. The uncertainties in the DM direct detection rates due to uncertainties in the Yukawa couplings will thus be negligible, assuming NFC. Note that the Higgs portal with an additional SM singlet mixing with the Higgs is also described by the above modifications of fermion couplings, with a completely universal shift $\kappa_{i}=\cos \theta$, where $\theta$ is the singlet-Higgs mixing angle $[199,200]$.

Our analysis of modified Higgs-portal DM phenomenology given in Section 4.2 applies in the somewhat special limit where the DM only couples to the lightest mass- 
eigenstate $h$. For instance, for scalar DM the general Higgs portal is

$$
\mathcal{L}_{\mathrm{NFC}}=g_{\chi, i j} \chi^{\dagger} \chi H_{i}^{\dagger} H_{j} .
$$

If the hermitian matrix of couplings $g_{\chi, i j}$ is such that it has $h$ as the only eigenstate with nonzero eigenvalue, then our analysis in Section 4.2 applies unchanged. In general, however, all the expressions in Section 4.2 get corrected by terms of order $1 / m_{H_{i}}^{2}$ due to exchanges of heavy Higgs bosons with masses $m_{H_{i}}$. If DM is heavy, $m_{\chi}>m_{H_{i}}$, the presence of heavy Higgs bosons would also open new annihilation channels.

\subsubsection{Type-II Two-Higgs-Doublet Model}

The MSSM tree-level Higgs potential and the couplings to quarks are the same as in the type-II two-Higgs-doublet model (2HDM), see, e.g., [201]. This is an example of a $2 \mathrm{HDM}$ with natural flavor conservation in which $v_{u}=\sin \beta v_{W}, v_{d}=\cos \beta v_{W}$. The mixing of $h_{u, d}$ into the Higgs mass-eigenstates $h$ and $H$ is given by

$$
\left(\begin{array}{l}
h_{u} \\
h_{d}
\end{array}\right)=\left(\begin{array}{cc}
\cos \alpha & \sin \alpha \\
-\sin \alpha & \cos \alpha
\end{array}\right)\left(\begin{array}{l}
h \\
H
\end{array}\right),
$$

where $h$ is the observed SM-like Higgs. Thus

$$
\begin{aligned}
& \kappa_{u}=\kappa_{c}=\kappa_{t}=\frac{\cos \alpha}{\sin \beta}, \\
& \kappa_{d}=\kappa_{s}=\kappa_{b}=-\frac{\sin \alpha}{\cos \beta},
\end{aligned}
$$

while the flavor-violating and/or $C P$-violating Yukawas are zero. In the decoupling limit $(\beta-\alpha=\pi / 2)$ the heavy Higgs bosons become infinitely heavy, while the Yukawa couplings tend toward their SM value, $\kappa_{i}=1$. The global fits to Higgs data in type-II 2HDM already constrain $\beta-\alpha \simeq \pi / 2[171,202,203]$ so that in this case the corrections to 
Higgs-portal DM phenomenology due to non-standard Higgs Yukawa couplings are small.

As in the case of MHDM, the DM phenomenology of Section 4.2 remains unchanged only in the limit where the DM couples to the light Higgs $h$ but not to the heavy Higgs $H$. In the general case, our analysis gets corrections that are relatively suppressed by $\mathcal{O}\left(m_{h}^{2} / m_{H}^{2}\right)$. If we are not too far away from the decoupling limit these corrections can be neglected, while in parts of the parameter space, where cancellation can occur, the extra contributions are numerically important [154].

\subsubsection{Higgs-dependent Yukawa Couplings}

In the model of quark masses introduced by Giudice and Ledebev (GL) [185] the Higgsquark interactions are written in terms of effective operators

$$
\mathcal{L}_{q}=c_{i j}^{u}\left(\frac{H^{\dagger} H}{M^{2}}\right)^{n_{i j}^{u}} \bar{Q}_{L, i} u_{R, j} H^{c}+c_{i j}^{d}\left(\frac{H^{\dagger} H}{M^{2}}\right)^{n_{i j}^{d}} \bar{Q}_{L, i} d_{R, j} H+\text { h.c. }
$$

They can be thought of as arising from integrating out heavy mediators at a large mass scale $M$. In this model the light quarks couple to the Higgs only through operators with mass dimension higher than four, i.e., for light quarks we have $n_{i j}^{u, d} \neq 0$. The values of the integers $n_{i j}^{u, d}$, and of the coefficients $c_{i j}^{u, d}$ that take values of order unity, are chosen such that the hierarchies of the observed quark masses and mixing angles are explained, after EWSB, in terms of the expansion parameter $\epsilon \equiv v_{W}^{2} / M^{2} \approx 1 / 60$. Thus, the Yukawa couplings are of the form

$$
y_{i j}^{u, d}=\left(2 n_{i j}^{u, d}+1\right)\left(y_{i j}^{u, d}\right)_{\mathrm{SM}} .
$$


After mass diagonalization the SM Yukawas are diagonal in the same basis as the quark masses, $\left(y_{i j}^{u, d}\right)_{\mathrm{SM}} \propto \delta_{i j} m_{i}^{u, d}$, while the $y_{i j}^{u, d}$ are not diagonal in the same basis ${ }^{3}$. Using the ansatz $n_{i j}^{u, d}=a_{i}+b_{j}^{u, d}$ with $a=(1,1,0), b^{d}=(2,1,1)$, and $b^{u}=(2,0,0)$, this gives the deviations in the Yukawa couplings collected in Tables 4.1-4.4 in the row denoted by "GL". Since the couplings to the bottom quark is enhanced by a large factor, $\kappa_{b} \simeq 3$, the simplest version of the GL model is already excluded by the Higgs data on $h \rightarrow W W$, $h \rightarrow Z Z$ and $h \rightarrow \gamma \gamma$ decays.

We therefore modify the initial GL proposal and assume that we have two Higgs doublets in (4.44), $H_{u}$ that only gives masses to up-type quarks and $H_{d}$ that only gives masses to down-type quarks. The correct mass and CKM angle hierarchy is obtained by using $b^{d}=(1,0,0)$ in the ansatz for $n_{i j}^{d}$, and leaving $a$ and $b^{u}$ unchanged. This gives satisfactory Higgs phenomenology at present as long as $\kappa_{b}=\sin \alpha / \cos \beta \simeq 1$ up to $\mathcal{O}(20 \%)$. In this limit also $\kappa_{t}=\cos \alpha / \sin \beta \simeq 1$. The scaling of Yukawa couplings for this modification of the GL model is shown in Tables 4.1-4.4 in the row denoted by "GL2".

In the GL model it is natural that the Higgs is the only state that couples to DM. The GL model is thus an example of Higgs-portal DM where the light-quark Yukawa couplings can substantially differ from their SM values. For instance, in GL2 $\kappa_{u} \simeq 7 \kappa_{t}$, $\kappa_{d} \simeq 5 \kappa_{b}, \kappa_{s} \simeq 3 \kappa_{b}, \kappa_{c} \simeq 3 \kappa_{t}$. The coupling of DM to gluons (4.25) $\mathcal{C}_{g} \simeq 4 \kappa_{t}+\kappa_{b}$, so that $\mathcal{C}_{g} \sim(5 / 3) \mathcal{C}_{g}^{\mathrm{SM}}$, and $\tilde{\mathcal{C}_{g}} \sim \mathcal{O}\left(\mathcal{C}_{g}\right)$. Taking $\kappa_{b} \simeq 1$, this means that the effective Higgs coupling to nucleons, governing the direct DM detections rates, gets enhanced compared to the SM Higgs Yukawa couplings by

$$
\frac{f_{\mathcal{S}}^{(p)}}{\left.f_{\mathcal{S}}^{(p)}\right|_{\mathrm{SM}}} \simeq 1.2 \kappa_{t}+1.3 \kappa_{b} \simeq 2.5, \quad \frac{f_{\mathcal{S}}^{(n)}}{\left.f_{\mathcal{S}}^{(n)}\right|_{\mathrm{SM}}} \simeq 1.3 \kappa_{t}+1.3 \kappa_{b} \simeq 2.6 .
$$

\footnotetext{
${ }^{3}$ Note that the mixing of contributions from different effective operators that may have large relative phases could lead to sizeable $\mathrm{CP}$-violating contributions to the Yukawa couplings.
} 
Here most of the enhancement over the SM comes from enhanced $\kappa_{u}$ and $\kappa_{d}$, which is also the reason for enlarged isospin breaking (the difference between $f_{\mathcal{S}}^{(p)}$ and $f_{\mathcal{S}}^{(n)}$ ). As a result of larger couplings to light quarks the spin-independent DM scattering cross section can thus be enhanced by an order of magnitude in the GL2 model of light-quark masses.

\subsubsection{Randall-Sundrum models}

The Randall-Sundrum (RS) warped extra-dimensional models with the SM fields propagating in the bulk provide a solution to the hierarchy problem and simultaneously explain the hierarchy of the SM fermion masses without large hierarchies in the initial five-dimensional (5D) Lagrangian [186, 204, 205]. The fermion zero modes are either localized toward the UV brane (for lighter fermions) or toward the IR brane (the top, the left-handed $b$ quark and potentially the right-handed $c$ quark) [206,207]. The Higgs field and the Higgs vev are both localized toward the IR brane. Integrating out the Kaluza-Klein (KK) modes and working in the limit of a brane-localized Higgs, the SM quark mass matrices are given, to leading order in $v_{W}^{2} / m_{K K}^{2}$, by [208] (see also [209-217], and Ref. [218] for a bulk Higgs scenario)

$$
M_{i j}^{d(u)}=\left[F_{q} Y_{1(2)}^{5 D} F_{d(u)}\right]_{i j} v_{W}
$$

Here, $m_{K K}$ is the KK mass scale. The $F_{q, u, d}$ are diagonal $3 \times 3$ matrices of fermion wave-functions for the left-handed electroweak quark doublets and the right-handed electroweak up and down quark singlets, respectively, evaluated at the IR brane. Assuming flavor anarchy, the 5D Yukawa matrices for up and down quarks, $Y_{1,2}^{5 D}$, are general $3 \times 3$ complex matrices with $\mathcal{O}(1)$ entries. For a Higgs field propagating in the bulk, 5D gauge invariance guarantees $Y_{1}^{5 D}=Y_{2}^{5 D}$ [208].

At leading order in $v_{W}^{2} / m_{K K}^{2}$ the Higgs Yukawas are aligned with the quark masses, 
i.e.,

$$
M_{u, d}=y_{u, d} \frac{v_{W}}{\sqrt{2}}+\mathcal{O}\left(v_{W}^{2} / m_{K K}^{2}\right) .
$$

The misalignment arises from dimension-six operators that are generated by tree-level KK quark exchanges. They give

$$
\left[y_{u(d)}\right]_{i j}-\frac{\sqrt{2}}{v_{W}}\left[M_{u, d}\right]_{i j} \sim-\frac{2}{3} F_{q_{i}} \bar{Y}^{3} F_{u_{j}\left(d_{j}\right)} \frac{v_{W}^{2}}{m_{K K}^{2}},
$$

where $\bar{Y}$ is a typical value of the dimensionless 5D Yukawa coupling and is in numerical analyses typically taken to be below $\bar{Y} \lesssim 4$ (see, e.g., [212]). The Higgs mediated FCNCs are thus suppressed by the same zero-mode wave-function overlaps that also suppress the quark masses, giving rise to the RS GIM mechanism [219-221].

Using that the CKM matrix elements are given by $V_{i j} \sim F_{q_{i}} / F_{q_{j}}$ for $i<j$, Eq. (4.49) can be rewritten as

$$
\left[y_{u(d)}\right]_{i j}-\frac{\sqrt{2}}{v_{W}}\left[M_{u, d}\right]_{i j} \sim-\frac{2}{3} \bar{Y}^{2} \frac{v_{W}^{2}}{m_{K K}^{2}}\left\{\begin{array}{cc}
\frac{m_{u_{j}\left(d_{j}\right)}}{v_{W}} V_{i j}, & i<j, \\
1, & i=j, \\
\frac{\left.m_{u_{i}\left(d_{i}\right)}\right)}{v_{W}} V_{i j}^{-1}, & j<i .
\end{array}\right.
$$

This yields the $\kappa_{i}$ collected in Tables 4.1-4.4. In the numerical analysis of ref. [208] the diagonal values $\kappa_{i}$ were typically found to be smaller than one, with deviations in $\kappa_{t}$ up to $30 \%, \kappa_{b}$ up to $15 \%$, in $\kappa_{s, c}$ up to $\sim 5 \%$, and in $\kappa_{u, d}$ of $1 \%$ (these estimates were obtained fixing the mass of the first $\mathrm{KK}$ gluon excitation to $3.7 \mathrm{TeV}$, above the present ATLAS bound [222]). The effective Higgs coupling to nucleons, $f_{\mathcal{S}}^{(N)}$, thus only gets reduced by $\mathcal{O}(10 \%)$, giving a $\mathcal{O}(20 \%)$ smaller DM scattering cross section on nuclei, compared to the case of SM Yukawa couplings. The largest effect arises in DM annihilations to top quarks, where the cross section can be reduced by a factor of two, while the annihilation cross section to $b \bar{b}$ pairs can be $\sim 30 \%$ smaller than for SM 
Yukawa couplings.

\subsubsection{Composite pseudo-Goldstone Higgs}

Finally, we investigate the possibility that the Higgs is a pseudo-Goldstone boson arising from the spontaneous breaking of a global symmetry in a strongly coupled sector [187-190]. We assume that the SM fermions couple linearly to composite operators $O_{L, R}[223]$,

$$
\lambda_{L, i}^{q} \bar{Q}_{L, i} O_{R}^{i}+\lambda_{R, j}^{u} \bar{u}_{R, j} O_{L}^{j}+\text { h.c. },
$$

where $i, j$ are flavor indices. This is the $4 \mathrm{D}$ dual of the fermion mass generation in $5 \mathrm{D}$ RS models. The Higgs couples to the composite sector with a typical coupling $y_{*}$. The SM masses and Yukawa couplings then arise from expanding the two-point functions of the $O_{L, R}$ operators in powers of the Higgs field [224], giving rise to four- and higherdimensional Higgs operators, such as in (4.35).

The new ingredient, related to the pNGB nature of the Higgs, is that the shift symmetry dictates the form of the higher-dimensional operators. The flavor structure and the composite Higgs coset structure completely factorize if the SM fields couple to only one composite operator. The general decomposition of Higgs couplings then becomes [224] (see also [225,226])

$$
Y_{u} \bar{Q}_{L} H u_{R}+Y_{u}^{\prime} \bar{Q}_{L} H u_{R} \frac{\left(H^{\dagger} H\right)}{\Lambda^{2}}+\ldots \quad \rightarrow \quad c_{i j}^{u} P(h / f) \bar{Q}_{L}^{i} H u_{R}^{j}
$$

and similarly for the down quarks. Here $P(h / f)=a_{0}+a_{2}(h / f)^{2}+\ldots$ is an analytic function whose form is fixed by the structure of the spontaneous breaking and the embedding of the SM fields in the global symmetry of the strongly coupled sector, while $f$ is the equivalent of the pion decay constant and is of order $v_{W}$. Since the flavor structure of the coefficients of the dimension-four and dimension-six operators is the 
same, they can be diagonalized simultaneously. All corrections to the quark Yukawa couplings from this effect are therefore strictly diagonal, and we have

$$
\kappa_{q} \sim 1+\mathcal{O}\left(\frac{v_{W}^{2}}{f^{2}}\right)
$$

For example, for the models based on the breaking of $S O(5)$ to $S O(4)$, the diagonal Yukawa couplings can be written as [227]

$$
\kappa_{q}=\frac{1+2 m-(1+2 m+n)\left(v_{W} / f\right)^{2}}{\sqrt{1-\left(v_{W} / f\right)^{2}}}
$$

where $n, m$ are positive integers. The MCHM4 model corresponds to $m=n=0$, while MCHM5 is given by $m=0, n=1$.

The flavor-violating contributions to the quark Yukawa couplings then arise only from corrections to the quark kinetic terms. That is, they are related to dimension-six operators of the form [224]

$$
\bar{q}_{L} i \not D q_{L} \frac{H^{\dagger} H}{\Lambda^{2}}, \bar{u}_{R} i \not D u_{R} \frac{H^{\dagger} H}{\Lambda^{2}}, \ldots
$$

These operators arise from the exchange of composite vector resonances with typical mass $M_{*} \sim \Lambda$. After using the equations of motion they contribute to the misalignment between the fermion masses and the corresponding Yukawa couplings. The NDA estimates for these corrections are, neglecting relative $\mathcal{O}(1)$ contributions in the sum $[161,224,228]$,

$$
\kappa_{i j}^{u} \sim 2 y_{*}^{2} \frac{v_{W}^{2}}{M_{*}^{2}}\left(\lambda_{L, i}^{q} \lambda_{L, j}^{q} \frac{m_{u_{j}}}{v_{W}}+\lambda_{R, i}^{u} \lambda_{R, j}^{u} \frac{m_{u_{i}}}{v_{W}}\right)
$$

and similarly for the down quarks. If the strong sector is $C P$ violating, then $\tilde{\kappa}_{i j}^{u, d} \sim \kappa_{i j}^{u, d}$. The exchange of composite vector resonances contributes also to the flavor diagonal 
Yukawa couplings, shifting the estimate (4.53) by (note the different normalizations of $\kappa_{q}$ and $\kappa_{q q^{\prime}}$ in (4.34))

$$
\Delta \kappa_{q_{i}} \sim 2 y_{*}^{2} \frac{v_{W}^{2}}{M_{*}^{2}}\left[\left(\lambda_{L, i}^{q}\right)^{2}+\left(\lambda_{R, i}^{u}\right)^{2}\right] .
$$

This shift can be large for the quarks with a large composite component if the Higgs is strongly coupled to the vector resonances, $y_{*} \sim 4 \pi$, and these resonances are relatively light, $M_{*} \sim 4 \pi v_{W} \sim 3 \mathrm{TeV}$. The left-handed top and bottom, as well as the right-handed top, are expected to be composite, explaining the large top mass (i.e., $\lambda_{L, 3}^{q} \sim \lambda_{R, 3}^{u} \sim$ 1). In the anarchic flavor scenario, one expects the remaining quarks to be mostly elementary (so the remaining $\lambda_{i} \ll 1$ ). However, if there is some underlying flavor alignment, it is also possible that the light quarks are composite. This is most easily achieved in the right-handed sector $[226,229,230]$. Taking all right-handed up-type quarks fully composite, and assuming that this results in a shift $\Delta \kappa_{u} \sim \Delta \kappa_{c} \sim \Delta \kappa_{t} \sim 1$, this would lead to an increase in the effective Higgs coupling to nucleons, $f_{\mathcal{S}}^{(N)}$, of about $50 \%$, and an increase in the DM-nucleon scattering rate of about $100 \%$.

\subsection{Constraining the light-quark Yukawa couplings}

If DM is a thermal relic interacting with ordinary matter predominantly via SM Higgs exchange, direct detection scattering rates immediately give information about the light-quark Yukawa couplings once the coupling of the DM particle to the Higgs particle is fixed.

In fact, DM scattering in direct detection searches would be one of the very few possible probes of the light-quark Yukawa couplings. The interactions of the Higgs boson with $u, d$, or $s$ quarks give rise to flavor-conserving neutral currents. Off-shell Higgs contributions in processes with only SM external particles always compete with other, much larger flavor-conserving neutral currents induced by gluon, photon, or 


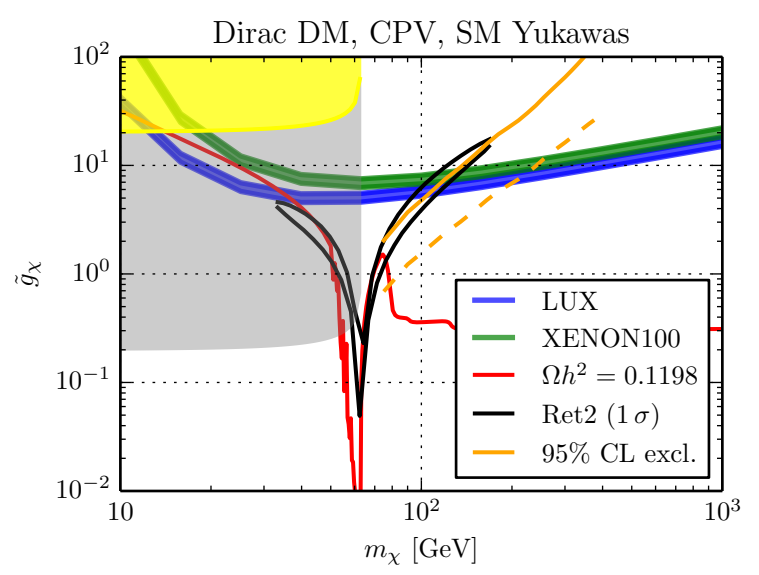

Figure 4.12: The $\gamma$-ray excess in the recently discovered dwarf spheroidal galaxy Reticulum 2, interpreted as a signal of DM annihilating into $b \bar{b}$ pairs, is shown as the black $1 \sigma$ contour (see Ref. [231] for details). The orange lines show the 95\% CL exclusion limits at the $14-\mathrm{TeV}$ LHC (solid line) and a prospective 100-TeV hadron collider (dashed line), obtained by rescaling the bounds given in Ref. [156]. The remaining color coding is the same as in Fig. 4.2. See text for more details.

$Z$ exchange. This leaves us with two options: either to consider on-shell Higgs decays [159-161], or to use new probes, such as DM scattering in direct detection experiments.

In principle, there is enough information to make a closed argument. Suppose that indirect DM searches yield a positive DM annihilation signal for $m_{\chi}>m_{h} / 2$. At the end of the high-luminosity LHC run, the Higgs couplings to $W, Z, t$, and $b$ will be precisely determined. Assuming that DM is a thermal relic interacting only through the Higgs portal, this fixes the value of $g_{\chi}$ since the annihilation cross section for $m_{\chi}>$ $m_{h} / 2$ is otherwise almost completely controlled by the Higgs couplings to $W, Z$, and $t$. In principle, a consistency check that the DM is really interacting through a Higgs portal could be provided, for a very limited range of DM masses $m_{\chi} \gtrsim m_{h} / 2$, by a 100-TeV hadron collider [156].

After the discovery of DM, the direct detection searches would immediately imply an upper bound on the light-quark Yukawa couplings. As an illustration, consider the 
excess in $\gamma$-ray emmission in the recently discovered dwarf spheroidal galaxy Reticulum 2 [178]. Let's take the bold step of interpreting this signal as originating from DM annihilating into $b \bar{b}$ pairs (see Ref. [231] for details). Assuming the Dirac-fermion DM scenario with purely CP-violating couplings, we obtain a $1 \sigma$ region in the $m_{\chi}-\tilde{g}_{\chi}$ plane that is not yet excluded by direct detection constraints, denoted by the orange lines in Fig. 4.12. Note that part of this region is consistent with DM furnishing the dominant component of the observed relic density while at the same time not being excluded by the invisible Higgs decay width. Concentrating on the overlap region, $m_{\chi} \sim 75 \mathrm{GeV}$, a comparison with the ratios shown in Fig. 4.11 would immediately imply an upper bound of $\kappa_{u} \lesssim 10, \kappa_{d} \lesssim 10, \kappa_{s} \lesssim 12$ from the LUX direct detection search (allowing only one of the Yukawa couplings to float at a time).

These estimates could potentially be loosened by uncertainties in the DM velocity profile and the local DM density. On the other hand, if DM is discovered in direct detection the relative size of the light-quark Yukawas could be probed by comparing scattering rates on different target materials.

An additional cross check of our scenario could be provided by searches for DM production at hadron colliders. In Fig. 4.12 we denote the 95\% CL exclusion limits, assuming $3000 / \mathrm{fb}$ of data, at the 14-TeV LHC by a solid orange line and at a prospective 100-TeV hadron collider by a dashed orange line. These curves have been obtained by converting the bounds in Ref. [156] to the case of Dirac DM using FeynRules [106] and MadGraph5 [232]. We see that, while the LHC will be sensitive to a part of the interesting region in parameter space, the scenario of $m_{\chi}=75 \mathrm{GeV}$ DM can be excluded only at a 100-TeV collider. 


\subsection{Chapter summary}

Not much is known experimentally about the couplings of the Higgs to light quarks. It is entirely possible that the Higgs couples only to the third generation of fermions. Experimentally equally viable is the possibility that the light-quark Yukawas are significantly enhanced, up to $\mathcal{O}(50)$ for $\kappa_{s}$ and up to $\mathcal{O}\left(10^{3}\right)$ for $\kappa_{u}$ and $\kappa_{d}$. Such extremely large enhancements are not natural from a model-building point of view as they require a large fine tuning of the light-quark masses, but at present cannot be excluded experimentally.

Modified Yukawa couplings to light quarks could have implications for DM searches. In this chapter, we focused on Higgs-portal DM. We considered constraints on scalar, vector, and fermionic Higgs-portal models of DM from relic density, direct and indirect detection, and the invisible Higgs width. A central result of our analysis is that, for phenomenologically viable Higgs-portal DM, there is a relatively small change in the predictions between the case where the Higgs is SM like and the case where the Higgs couples only to the third generation of fermions. For direct detection this is a consequence of the fact that, for very small light-quark Yukawas, the scattering cross section is dominated by the effective Higgs-gluon coupling, which is obtained by integrating out the heavy quarks. For instance, setting all the light quark Yukawa couplings to zero reduces the direct detection scattering cross section by $\sim 50 \%$ compared to the case where the light quark Yukawa couplings are assumed to have SM values. Similarly, the relic abundance and indirect detection signals are dominated by the heaviest kinematically open annihilation channels, diminishing the importance of Higgs couplings to light quarks.

On the other hand, saturating the experimentally allowed values for the light-quark Yukawas, the DM direct detection rates can increase by four orders of magnitude com- 
pared to the case where the light-quark Yukawa couplings are kept at their SM values. Moreover, negative values of the light-quark Yukawa couplings can result in a strong reduction of the nucleon scattering rates. The changes in DM annihilation rates are much smaller. The annihilation of DM into light quarks is a subleading effect, unless $m_{\chi}<m_{W}$. Even in this case, the dominant annihilation channel is into $b \bar{b}$ pairs, while the annihilation to light quarks can constitute at most an $\mathcal{O}(1)$ fraction if the current experimental upper bounds on the light-quark Yukawa couplings are saturated. A Higgs-portal for DM in this mass range is excluded either by bounds on the invisible Higgs decay width or by indirect DM searches.

We also investigated the expected sizes of corrections to DM phenomenology due to changes in Yukawa couplings in a number of new physics models. The largest deviation in expected DM scattering rate on nucleons was found for a modified GiudiceLebedev model of light-quark masses where up to an order of magnitude enhancement due to corrections to light-quark Yukawa couplings are possible. Similarly, an $\mathcal{O}(1)$ change of the scattering rate is anticipated in a pseudo-Goldstone Higgs scenario with composite right-handed light quarks while in RS models with anarchic flavor a reduction of about $20 \%$ can be expected. The effects in MFV models, multi-Higgs models with natural flavor conservation, and the type-II two-Higgs-doublet model (i.e., the tree-level Higgs sector of the MSSM), on the other hand, are expected to be much smaller.

Finally, we point out that a discovery of Higgs-portal DM in indirect searches would immediately imply an upper bound on the light-quark Yukawa couplings due to the upper bounds in direct DM searches. 


\section{Chapter 5}

\section{Probing CP Violation in $h \rightarrow \gamma \gamma$ with}

\section{Converted Photons}

\subsection{Introduction}

The recent discovery of a Higgs-like boson [233,234] has prompted intense interest in the precise measurement of its couplings and properties. Such measurements are a direct probe of new physics (NP) beyond the Standard Model (SM), especially since many extensions of the SM modify the Higgs couplings to gauge bosons and fermions.

Of particular interest is a search for parity and $\mathrm{CP}$ violating Higgs decays, since these would be a clear signal of NP [172,235-251]. In Higgs decays to vector bosons the $\mathrm{CP}$ violating effects can only be due to irrelevant NP operators. Fortunately, in the $\mathrm{SM} h \rightarrow \gamma \gamma$ (and $h \rightarrow Z \gamma$ ) decays are also due to irrelevant operators, with the first non-zero contribution occurring at one-loop. Thus we can expect large $\mathrm{CP}$ violating effects from weak scale NP in $h \rightarrow \gamma \gamma$. In contrast, the $h \rightarrow Z Z^{*}$ and $h \rightarrow W W^{*}$ decays proceed in the SM through relevant tree-level operators tightly related to the $Z$ and $W$ masses. $\mathrm{CP}$ violating effects from NP are expected to be comparatively small in these decay modes. 
In the presence of CPV, the total $h \rightarrow \gamma \gamma$ decay rate must be proportional to the sum of squares of CP-even and CP-odd terms - i.e $\left|\mathrm{CP}_{\text {even }}\right|^{2}+\left|\mathrm{CP}_{\text {odd }}\right|^{2}-$ and therefore, by comparing the $h \rightarrow \gamma \gamma$ rate to the SM expectation, one may probe for NP directly. However, this type of search cannot distinguish between $\mathrm{CP}$-even and CP-odd NP contributions to the total rate. Moreover if the $\mathrm{CP}$-odd contribution is small, then $\mathrm{CPV}$ signals are quadratically suppressed, and if it so happens that NP enters both the CP even and odd terms, such that the total $h \rightarrow \gamma \gamma$ rate matches the SM expectation, then one cannot detect NP at all. Probing the differential $h \rightarrow \gamma \gamma$ rate for CPV ameliorates these problems. In the first instance, the differential rate may feature an interference term of the form $2 \mathrm{CP}_{\text {even }} \mathrm{CP}_{\text {odd }}$. Combined with non-interfering terms, one may distinguish CP-even and CP-odd NP contributions. Secondly, small CPV signals are only linearly suppressed in this interference term.

The $h \rightarrow \gamma \gamma$ phase space distribution alone, however, is not sensitive to CP violating effects, since the Higgs decays isotropically to two photons. Nevertheless, the underlying CPV structure in the differential $h \rightarrow \gamma \gamma$ rate may be determined if one is able to measure the linear polarizations of the outgoing photons. This in itself is an old idea, first proposed for the determination of the $\pi^{0}$ parity [252-254] and, more recently, to probe NP effects in radiative $B$ decays [255]. It relies on the fact that a spin-0 particle decays to either two positive or two negative helicity photon states, which acquire a relative $\mathrm{CPV}$ phase in the presence of non-trivial $\mathrm{CP}$ structure. The linearly polarized photon states are a superposition of both helicities, permitting one to extract this CPV phase. It is not feasible to directly measure the linear polarization of $\mathcal{O}(60 \mathrm{GeV})$ photons from Higgs decay. However, in both the ATLAS [256] and CMS [257] detectors roughly half of the photons from Higgs decays convert via the well-known Bethe-Heitler (BH) process into $e^{+} e^{-}$pairs inside the silicon tracker. This has an important benefit: the orientation of the produced $e^{+} e^{-}$pairs encodes the underlying CP structure. Figure 5.1 illustrates an observable expected to be sensitive to 


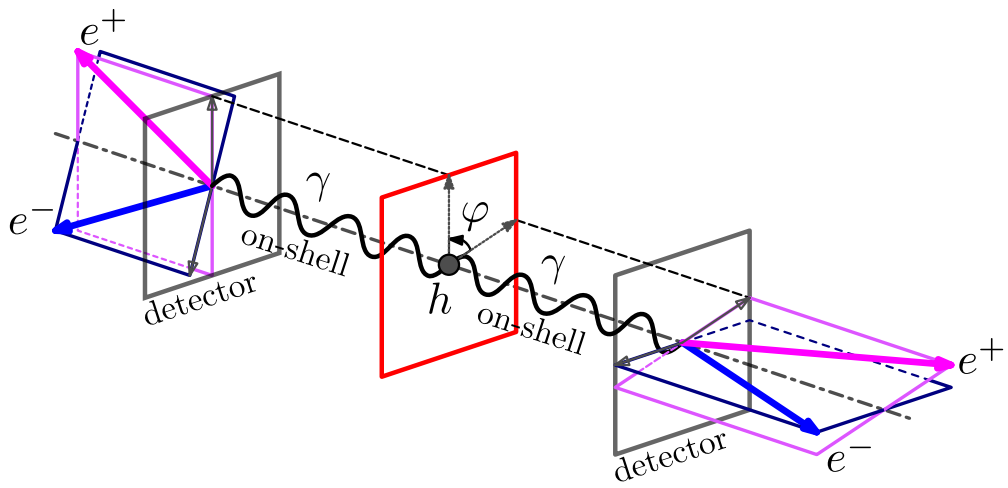

Figure 5.1: An illustration of an example of a CPV sensitive observable in $h \rightarrow \gamma \gamma \rightarrow 4 e$. The Higgs decays to on-shell photons which convert in the detector. The distribution of the azimuthal angle $\varphi$ between the two planes formed by each positron and its parent photon depends on the Higgs couplings to $\mathrm{CP}$ even and odd operators. The electrons do not need to be co-planar with the corresponding photon-positron planes. The positron-photon plane is shown in magenta and the electron-photon plane in blue. For further details and subtleties see the main text.

CPV.

Previous proposals to measure CPV in $h \rightarrow \gamma \gamma$, or in other neutral meson diphoton decays, via double photon conversion appear in Refs. [235,254,258]. We extend these studies by performing an analysis of the actual manner in which the leptonic phase space encodes the $\mathrm{CP}$ violating effects. We examine the encoding of CPV in the doubly converted Higgs diphoton decay - hereafter the Higgs-Bethe-Heitler $(\mathrm{HBH})$ process - both analytically and numerically, making use of the spinor-helicity formalism to obtain compact expressions for the full differential scattering rate and its leading order terms.

A key difficulty in extracting CPV signals from differential scattering rates like $d \Gamma / d \varphi$, defined in Fig. 5.1, is that the signal is largely washed out under integration over the other phase space variables. However, in this work we use our analytic control of the full differential scattering rate to show that large CPV signals may be achieved. We find certain observables exhibit $\mathcal{O}(1)$ sensitivity to $\mathrm{CP}$ violating effects on small regions of the phase space, corresponding to a small fraction of the converted decays. 
These regions are identified by sensitivity parameters, derived from our analysis. We show analytically and confirm numerically that if one cuts the $\mathrm{HBH}$ event data according to these sensitivity parameters, the CPV signal is dramatically improved.

Performing such an experiment will be difficult. For example, one must resolve the opening angles in the photon conversion, and this requires extremely accurate tracking resolution. Other subtle effects, such as soft scatterings of the electron and positron in the detector material need also to be examined. In addition, reaching a sensitivity to Higgs couplings would require obtaining a signal-rich sample of $h \rightarrow \gamma \gamma$ events. Our approach here is to defer these considerations to future work, and consider mainly the theoretical aspects of this process. In doing this, our intent is to motivate a very challenging measurement, perhaps to be done after the LHC upgrade or in a future Higgs factory. We note however, that as a warm-up to Higgs studies, photon conversions can be studied in background samples as a test of the standard model (we present the phase space structure of $\bar{q} q \rightarrow \gamma \gamma$ with converted photons in an appendix).

This chapter is structured as follows. In Section 5.2 we first present a motivating phenomenological analysis of the expected size of CP violation in Higgs decays, followed by an analysis of the unconverted $h \rightarrow \gamma \gamma$ process. This is presented in the language of helicity amplitudes and we show that the CPV terms in the differential rate arise from helicity amplitude interference. In Section 5.3 we discuss photon propagation and conversion in modern detectors, angular resolution limits, and the central role of the nuclear form factor in Bethe-Heitler conversion. In Section 5.4 we then proceed to examine the $\mathrm{HBH}$ process itself, presenting explicit results within the spinor-helicity formalism for the leading order HBH square amplitude. This is followed in Section 5.5 by a derivation of CPV observables and their sensitivity parameters for the special case that the Higgs is at rest. These sensitivity parameters can be used as cuts, which extract the phase space regions on which we expect $\mathcal{O}(1) \mathrm{CPV}$ effects. Numerical simulations are presented which confirm these expectations, and further compare the performance 
of the various sensitivity parameter cuts. In this work we focus on the $h \rightarrow \gamma \gamma$ decay, but our analysis can be used to examine other searches, e.g., for $h \rightarrow Z \gamma$ or other decays involving converted photons.

\subsection{Higgs diphoton decay with $\mathrm{CP}$ violation}

\subsubsection{Motivation for measuring CP-violation in di-photons}

The CP structure of Higgs decays was already studied experimentally in $h \rightarrow Z Z^{*}$ decays [259,260], with pure pseudo-scalar coupling disfavored at more than $3 \sigma$ [260]. Still, there is strong motivation to measure CPV also in loop induced $h \rightarrow \gamma \gamma$ decays. The motivation is based on the expected sizes of $\mathrm{CP}$ violating and $\mathrm{CP}$ conserving terms in $h \rightarrow \gamma \gamma$ and $h \rightarrow Z Z^{*}$ decays. After electroweak symmetry breaking (EWSB), the relevant terms in the effective Lagrangian at the scale $\mu \simeq m_{h} / 2$ are

$$
\begin{gathered}
\mathcal{L}_{\text {eff }} \supset c_{V} \frac{m_{Z}^{2}}{v} h Z^{\mu} Z_{\mu}+c \frac{\alpha}{\pi v} h F_{\mu \nu} F^{\mu \nu}+c_{Z Z} \frac{\alpha}{\pi v} h Z^{\mu \nu} Z_{\mu \nu} \\
+\tilde{c}_{Z Z} \frac{\alpha}{2 \pi v} h Z_{\mu \nu} \tilde{Z}^{\mu \nu}+\tilde{c} \frac{\alpha}{2 \pi v} h F_{\mu \nu} \tilde{F}^{\mu \nu}
\end{gathered}
$$

in which $F_{\mu \nu}$ and $Z_{\mu \nu}$ are respectively the photon and $Z$ field strengths, and $\tilde{X}^{\mu \nu}=$ $\epsilon^{\mu \nu \alpha \beta} X_{\alpha \beta}, \epsilon^{0123}=1$, while $v=246 \mathrm{GeV}$ is the Higgs vev. Taking Higgs to be a scalar, the first line of Eq. (6.2) contains CP even and the second line CP odd operators. ${ }^{1}$ Present data imply $c_{V}=1.04 \pm 0.13$ [261], assuming CP conservation. If either $\tilde{c}_{Z Z}$ or $\tilde{c}$ are found to be non-zero, ${ }^{2} \mathrm{CPV}$ in Higgs couplings and thus NP will be discovered. The couplings $c$ and $\tilde{c}$ arise at one-loop in perturbative UV theories, and can be at most

\footnotetext{
${ }^{1}$ Since all the couplings are $\mathrm{C}$ even, $\mathrm{P}$ and $\mathrm{CP}$ violation are the same. While we hereafter always refer to $\mathrm{CP}$ violation, it should be kept in mind that it is equivalent to parity violation for this effective theory.

${ }^{2}$ In fact, $\tilde{c}$ may be non-zero at three loops in the SM [262]. However, since this contribution falls well below the feasible detection threshold for the experiments under consideration in this work, we neglect this contribution, and treat the SM value as $\tilde{c}=0$.
} 
$\mathcal{O}(1)$ in order to agree with the observed $h \rightarrow \gamma \gamma$ rate. For example, $\tilde{c}$ can arise from a massive NP fermion loop that is axially coupled to the Higgs. Note that in Eq. (6.2) we integrated out both $W$ and $t$ loop contributions to $c$, so that $c_{\mathrm{SM}}=-0.81$ in the SM. Similarly, the dimension 5 couplings of Higgs to $Z Z, c_{Z Z}$ and $\tilde{c}_{Z Z}$, also arise at one-loop order. In generic NP models we thus expect $c \sim c_{Z Z}$ and $\tilde{c} \sim \tilde{c}_{Z Z}$.

The $h \rightarrow Z Z^{*}$ decay is dominated by the $\mathrm{CP}$ even renormalizable coupling $c_{V}$, while the $h \rightarrow \gamma \gamma$ decay is given by higher dimensional operators. The relative size of $\mathrm{CP}$ violating effects in any channel is given by the ratio of the interference terms to the total amplitude squared. For $h \rightarrow Z Z^{*}$ the $\mathrm{CP}$ odd interference term is proportional to $(\alpha / 2 \pi) \tilde{c}_{Z Z} c_{V}$, while the total squared amplitude is dominated by $c_{V}^{2}$. The typical size of CPV observables in $h \rightarrow Z Z^{*}$ is therefore set by the ratio of the two,

$$
r_{Z Z^{*}}=\frac{\alpha}{2 \pi} \frac{\tilde{c}_{Z Z}}{c_{V}} \sim \mathcal{O}\left(10^{-3}\right)
$$

for $\mathcal{O}(1)$ couplings. In the diphoton channel both terms are loop suppressed and the figure of merit for $\mathrm{CP}$ violation is

$$
r_{\gamma \gamma}=\frac{c \tilde{c}}{c^{2}+\tilde{c}^{2}} \sim \mathcal{O}(1)
$$

again assuming $\mathcal{O}(1)$ couplings. As will become clear, the measurement of $\mathrm{CPV}$ in $h \rightarrow \gamma \gamma$ is a challenging one, especially in comparison to the relatively straightforward measurement in $h \rightarrow Z Z^{*}$. However, the expected size of the effect may partially compensate for this.

In addition to the CPV observables discussed in this chapter, the CPV operator in Eq. (6.2) also modifies the overall $h \rightarrow \gamma \gamma$ decay rate, so that

$$
\mu_{\gamma \gamma} \equiv \frac{\Gamma(h \rightarrow \gamma \gamma)}{\Gamma(h \rightarrow \gamma \gamma)_{\mathrm{SM}}}=\frac{c^{2}+\tilde{c}^{2}}{c_{\mathrm{SM}}^{2}}
$$


where $\Gamma(h \rightarrow \gamma \gamma)_{\mathrm{SM}}$ is the SM rate. The total rate is only quadratically sensitive to $\mathrm{CP}$ violating NP because the interference terms integrate to zero over phase space.In contrast, the differential rates contain $\mathrm{CP}$ odd terms proportional to $c \tilde{c}$ and thus may be linear in the NP coupling. This can lead to substantial increase in sensitivity for small $\tilde{c}$.

Before proceeding, two remarks are in order. First, it is important to mention that there are severe constraints on $y_{e} \tilde{c}$ from bounds on the electric dipole moment of the electron [263-265]. Taking the electron yukawa, $y_{e}$, to be the SM one, this gives $\tilde{c} \lesssim$ $10^{-3}$. These bounds are, however, absent in the limit where the $125 \mathrm{GeV}$ Higgs does not couple to electrons (for other possibilities in concrete UV models, see [263,266]). A strong motivation for contemplating a non-zero value of $\tilde{c}$ is, for instance, that it would be generated by new $\mathrm{CP}$ sources in models that lead to electroweak baryogenesis, see e.g., [266]. An independent measurement of $\tilde{c}$ is thus desirable.

Second, we assumed here that $c$ and $\tilde{c}$ are real. In the $\mathrm{SM} c$ obtains its dominant contribution from a $W$ loop and a smaller destructive contribution from a top quark loop. However, there is also a smaller contribution from $b$ quark and light quarks. These can go on-shell in the loop, generating complex effective couplings $c$ and $\tilde{c}$. This means that $c$ and $\tilde{c}$ obtain a relative strong phase. The result of such a strong phase, when combined with the weak phase, would be to induce direct CPV in decay, such that the decay rate of the Higgs into two positive helicity photons is not the same as into two negative ones. These strong phases are of $\sim \mathcal{O}(1 \%)$ in the SM $[267,268]$, and we assume the strong phases are similarly small for NP effects. Consequently, we neglect direct $\mathrm{CPV}$, and assume that $c$ and $\tilde{c}$ are real. 


\subsubsection{Helicity interference}

We proceed to examine the unconverted $h \rightarrow \gamma \gamma$ process. The effective operator mediating $h \rightarrow \gamma \gamma$ decay has the general form, cf. Eq. (6.2),

$$
\mathcal{H}_{\mathrm{eff}}=-c \frac{\alpha}{\pi v} h F_{\mu \nu} F^{\mu \nu}-\frac{\tilde{c}}{2} \frac{\alpha}{\pi v} h F_{\mu \nu} \tilde{F}^{\mu \nu} .
$$

For a Higgs that is a scalar, the first term is $\mathrm{CP}$ even and the second is $\mathrm{CP}$ odd. $\mathrm{CP}$ is therefore violated if the $\mathrm{CP}$ phase

$$
\xi \equiv \tan ^{-1}(\tilde{c} / c)
$$

is found to be non-zero.

The $h \rightarrow \gamma \gamma$ helicity amplitudes are (dropping the overall $\alpha / \pi v$ factor, cf. Eq. (5.5))

$$
\begin{aligned}
i \mathcal{M}^{\lambda_{1} \lambda_{2}} & =\cdots \cdots-v_{k_{2}, \lambda_{2}}^{k_{1}, \lambda_{1}} \\
& =c\left[\left(k_{1} \cdot k_{2}\right)\left(\left(\varepsilon_{1}^{\lambda_{1}}\right)^{*} \cdot\left(\varepsilon_{2}^{\lambda_{2}}\right)^{*}\right)-\left(k_{1} \cdot\left(\varepsilon_{2}^{\lambda_{2}}\right)^{*}\right)\left(k_{2} \cdot\left(\varepsilon_{1}^{\lambda_{1}}\right)^{*}\right)\right]+\tilde{c} \epsilon\left[k_{1},\left(\varepsilon_{1}^{\lambda_{1}}\right)^{*}, k_{2},\left(\varepsilon_{2}^{\lambda_{2}}\right)^{*}\right],
\end{aligned}
$$

where $k_{i}$ are the photon momenta, $\varepsilon_{i}^{\lambda_{i}}$ is the polarization vector of the $i$ th photon $(i=1,2)$ with helicity $\lambda_{i}= \pm$, and $\epsilon[p, q, r, s] \equiv p_{\mu} q_{\nu} r_{\rho} s_{\sigma} \epsilon^{\mu \nu \rho \sigma}$. A Latin subscript $i=1,2$ hereafter denotes the corresponding photon. To compute helicity amplitudes we employ the spinor-helicity formalism, see Appendix C.I for our conventions and a brief review. Using Eqs. (C.5), (C.6) and (C.10), one finds that the non-zero helicity amplitudes are

$$
\mathcal{M}^{ \pm \pm}=m_{h}^{2}(c \pm i \tilde{c})=m_{h}^{2} \sqrt{c^{2}+\tilde{c}^{2}} e^{ \pm i \xi}
$$


while $\mathcal{M}^{ \pm \mp}=0$ as expected from angular momentum conservation (cf. also the results of, e.g., $[269,270]$ for $h \rightarrow Z Z$ ). We see that CPV introduces a relative phase, $\xi$, between the two-photon helicity amplitudes. Furthermore, a differential rate may depend on $\xi$ if and only if there is interference between $\mathcal{M}^{++}$and $\mathcal{M}^{--}$, or more precisely, between amplitudes involving the ++ and -- photon helicity configurations. We call such interference helicity interference.

Let us now translate Eq. (5.8) into a Hilbert space language. The final states of $h \rightarrow \gamma \gamma$ decay are the two-photon states $|++\rangle$ and $|--\rangle$, with \pm indicating the helicity of each photon, so that $\mathrm{CP}| \pm \pm\rangle=|\mp \mp\rangle$. Eq. (5.8) then translates to

$$
\mathcal{H}_{\text {eff }}|h\rangle \propto \sqrt{c^{2}+\tilde{c}^{2}}\left(e^{i \xi}|++\rangle+e^{-i \xi}|--\rangle\right) .
$$

As above, the $\mathrm{CP}$ phase $\xi$ appears as a relative phase between the $|++\rangle$ and $|--\rangle$ terms.

Now, the total rate for $h \rightarrow \gamma \gamma$ decay is proportional to

$$
\sum_{f=++,--}\left|\left\langle f\left|\mathcal{H}_{\mathrm{eff}}\right| h\right\rangle\right|^{2}=\sum_{f=++,--}\left|\mathcal{M}^{f}\right|^{2}
$$

Orthogonality of $|++\rangle$ and $|--\rangle$ ensures that the total rate is independent of $\xi$, i.e., there is no helicity interference. In contrast, any experiment for which the final state is a linear superposition of the two helicity states would generate helicity interference. This is the case at collider experiments in which the on-shell photons with definite helicity are intermediate states: The final state is a converted photon - an $e^{+} e^{-}$pair with a particular set of momenta - which has non-vanishing overlap with both helicities.

\subsubsection{A thought experiment with polarizers}

The overlap of each photon helicity with a BH pair will determine the strength of helicity interference and our ultimate sensitivity to the Higgs $\mathrm{CP}$ properties. The details at 


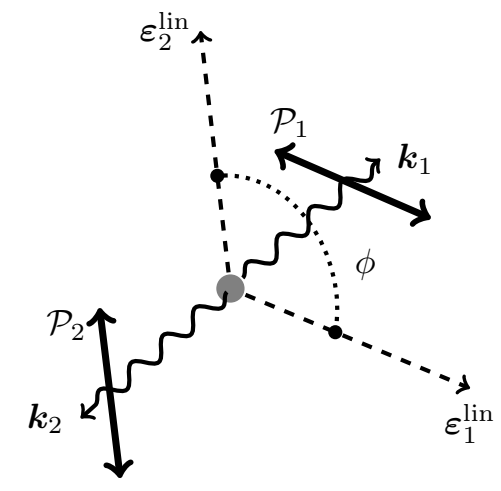

Figure 5.2: A linear polarization thought experiment in Higgs rest frame. $\mathcal{P}_{1,2}$ are linear polarizers oriented orthogonal to the photon momentum direction. The angle $\phi$ is measured between the linear polarization vectors $(m) \varepsilon_{1,2}^{\text {lin }}$.

the level we need are quite involved, and will be described in Sec. 5.4. As a warm-up we instead consider a thought experiment in which we can measure linearly polarized photons.

Let us imagine that we have been able to manufacture a linear polarizer for gamma rays. We produce a Higgs at rest between polarizers $\mathcal{P}_{1}$ and $\mathcal{P}_{2}$, such that each photon travels through a polarizer (see Fig. 5.2) before being absorbed by a detector that counts photons. The polarizer $\mathcal{P}_{i}(i=1,2)$ projects an incoming photon onto a linearly polarized state, $\left|\phi_{i}\right\rangle=e^{-i \phi_{i}}|+\rangle+e^{i \phi_{i}}|-\rangle$, that has polarization oriented at angle $\phi_{i}$. From Eq. (5.9) the amplitude of the two-photon wave function observed by the detectors is

$$
\left(e^{i \phi}\langle+|+e^{-i \phi}\langle-|\right)_{2} \otimes\left(\left\langle+|+\langle-|)_{1} \mathcal{H}_{\mathrm{eff}} \mid h\right\rangle \sim \sqrt{c^{2}+\tilde{c}^{2}} \cos (\phi+\xi),\right.
$$

where $\phi=\phi_{1}-\phi_{2}$ is a relative azimuthal angle between the two polarizers. As the angle $\phi$ is changed, the differential rate in the detectors changes as $\cos ^{2}(\phi+\xi)$. One finds

$$
\frac{d \Gamma}{d \phi}=\frac{2}{\pi} \Gamma_{h \rightarrow \gamma \gamma} \cos ^{2}(\phi+\xi), \quad \Gamma_{h \rightarrow \gamma \gamma}=\frac{\alpha^{2}}{4 \pi^{3}} \frac{m_{h}^{3}}{v^{2}}\left(c^{2}+\tilde{c}^{2}\right) .
$$

Note that the CP odd term in the differential rate (5.12) is proportional to $\sin 2 \xi \sin 2 \phi \propto$ 
$c \tilde{c} \sin 2 \phi$. The differential rate is thus linearly sensitive to CPV coupling $\tilde{c}$, whereas the total rate is quadratically sensitive.

In summary, we have shown that only the terms receiving contributions from both of the definite helicity two-photon amplitudes, so that there is helicity interference, are sensitive to the CPV phase $\xi$. These interference effects can in principle be of $\mathcal{O}(1)$ in size.

\subsection{Bethe-Heitler photon conversion}

We now study the process that can be used for photon polarization measurement, namely the conversion of a photon into an $e^{+} e^{-}$pair in matter. In this section we study a conversion of single isolated photon, which we will then use in the Sec. 5.4 for the case of $h \rightarrow \gamma \gamma$ with converted photons.

\subsubsection{Photon propagation and conversion}

Photon conversion to $e^{+} e^{-}$pairs may proceed either by Dalitz conversion in vacuum, for an off-shell intermediate photon, or by $\mathrm{BH}$ conversion on atomic nuclei, which occurs for on-shell photons (for a review see, e.g., $[272,273]$ ). The Dalitz conversion rate carries a suppression factor of $\mathcal{O}\left(10^{-4}\right)$, and is not of immediate practical interest. Moreover it mainly proceeds via a longitudinal photon so that the above helicity analysis no longer applies. In contrast, the CMS and ATLAS pixel detectors contain a significant amount of material, so that $\sim 50 \%$ of photons convert inside the tracking systems via the $\mathrm{BH}$ process $[274,275]$. Based on the composition of the detectors, we assume in this work that the target nucleus is always ${ }^{28} \mathrm{Si}$, at rest in the laboratory frame. This nucleus is spin- 0 , and has no nuclear magnetic moment. We therefore do not consider the effects of target polarization on the $\mathrm{BH}$ process [276].

One might be concerned by the prospect of photon polarization decoherence for 


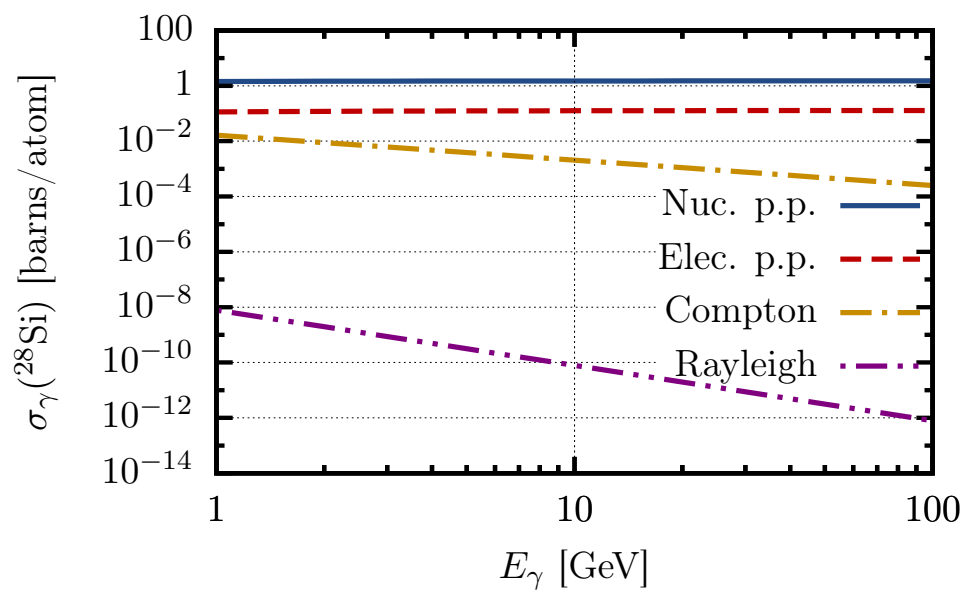

Figure 5.3: The contributions to photon cross-section on ${ }^{28} \mathrm{Si}, \sigma_{\gamma}\left({ }^{28} \mathrm{Si}\right)$, from $\mathrm{BH} e^{+} e^{-}$ pair production in nuclear field (solid blue line), pair production due to scattering on electron cloud (red dashed), Compton scattering (dot-dashed yellow) and Rayleigh scattering (magenta double dot-dashed), as a function of photon energy $E_{\gamma}$. Calculated using NIST's XCOM database [271].

the photons propagating inside the silicon. However, at photon energies $\sim \mathcal{O}\left(m_{h} / 2\right)$ the pair production in the nuclear field is by far the largest contribution to the photon scattering cross-section in an atomic lattice [277], see Fig. 5.3. As a result, to an excellent approximation, the photons remain coherent up until their $\mathrm{BH}$ conversion. We shall also assume that the $\mathrm{BH}$ scattering is quasi-elastic, i.e. that the target nucleus remains in a coherent state during and after the scattering. For the kinematics considered, the quasi-elastic limit is an excellent approximation of the full BH conversion [272,273].

\subsubsection{Angular resolution limitations}

Following the $h \rightarrow \gamma \gamma$ experiment discussed in Sec. 5.2.3, in order to measure the CPV in a doubly converted $h \rightarrow \gamma \gamma$ decay, we might expect that angular distributions between planes formed by spatial momenta need to be measured. There are several possible planar distributions that can be constructed, involving either:

i) The $e^{+} e^{-}$plane formed by the $e^{+} e^{-}$momenta, or; 


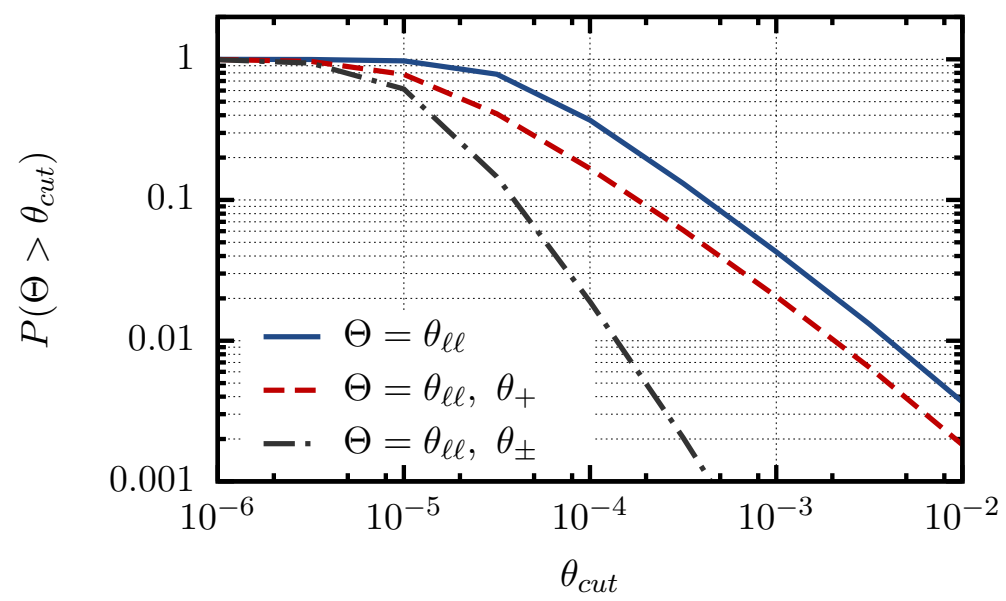

Figure 5.4: Cumulative distributions of Bethe-Heitler conversion cross-section for a photon with $E_{\gamma}=60[\mathrm{GeV}]$ scattering on a ${ }^{28} \mathrm{Si}$ nucleus, with respect to various opening angle cuts. Three distributions are shown: $P\left(\theta_{\ell \ell}>\theta_{\text {cut }}\right)$, i.e. with photon-lepton opening angles, $\theta_{ \pm}$, unconstrained (blue line); $P\left(\theta_{+}\right.$and $\left.\theta_{\ell \ell}>\theta_{\text {cut }}\right)$ with electronphoton opening angle $\theta_{-}$unconstrained (red dashed line); $P\left(\theta_{ \pm}\right.$and $\left.\theta_{\ell \ell}>\theta_{\text {cut }}\right)$ (black dot-dashed line).

ii) The $\gamma e^{ \pm}$plane formed by a lepton and its parent photon, as in Fig. 5.1.

The first requires resolving the orientation of the leptonic spatial momenta. The second requires the orientation of the leptons with respect to their parent photon, which could be achieved by identifying the vertex associated with the Higgs (from other tracks in the event) as well as the location of the photon conversion, giving the photon direction.

Such measurements require exquisitely precise tracking. Because the momentum transfer to the nucleus is small, the relative angular orientations between the photon and leptons are tiny in typical photon conversion: for mass $m$ and energy $E$, the angular scale is typically $m / E \sim 10^{-5}$ for a $60 \mathrm{GeV}$ photon conversion to electrons. There is however a distribution for these angles. In the limit of very large statistics one can hope to get a sample of events where these angles can be measured.

In the ATLAS detector, for instance, the intrinsic accuracy in silicon pixels located between $5 \mathrm{~cm}$ and $12 \mathrm{~cm}$ from the interaction point is $10 \mu \mathrm{m}$ in $R-\phi$ direction and $115 \mu \mathrm{m}$ in $z$ direction. The intrinsic accuracy of SCT strips located between $30 \mathrm{~cm}$ to $51 \mathrm{~cm}$ 
from the interactions point is $17 \mu m(R-\phi)$ and $580 \mu m(z)$ [278]. One may therefore hope to measure the orientations of the $e^{+} e^{-}$plane even for opening angles as small as $\theta_{\ell \ell} \sim 10^{-4}-10^{-3}$, where the relative leptonic angle $\theta_{\ell \ell}$ is defined by

$$
\cos \theta_{\ell \ell}=\frac{(m) p_{+} \cdot(m) p_{-}}{\left|(m) p_{+}\right|\left|(m) p_{-}\right|}
$$

for leptonic spatial momenta $(m) p_{ \pm}$. By comparison, a $60 \mathrm{GeV}$ photon converting to a $e^{-} e^{+}$pair has an opening angle of $\theta_{\ell \ell}>10^{-4}$ in $38 \%$ of the cases and $\theta_{\ell \ell}>10^{-3}$ in $4 \%$ of the cases. The full cumulative distribution for $\theta_{\ell \ell}>\theta_{\text {cut }}$, is shown in Fig. 5.4.

Another experimental challenge is the multiple scattering of outgoing electrons when traversing the detector medium. This can affect the measurement of the electron direction and thus the orientation of the $e^{+} e^{-}$or $\gamma e^{ \pm}$plane. Using Eq. (30.15) in [277], the width of the angular distribution is $\sim 10^{-4}$ for a $30 \mathrm{GeV}$ electron, assuming it traverses $\ell=0.1$ radiation lengths of the material. This width roughly scales as $\sqrt{\ell} / E$, where $E$ is the lepton energy. The measurement of polarization planes in the current and future detectors will thus be challenging, but may be achievable on a statistical basis. Bearing in mind these experimental questions, in the remainder of this chapter we adopt a theoretical approach to this problem: We consider a thought experiment where all angles can be resolved and explore the sensitivity to Higgs parameters in this best-case scenario.

\subsubsection{Nuclear form factor}

The BH conversion depends on a momentum transfer, $q^{\mu}$, between the photon and the nucleus. Assuming quasi-elastic scattering, the photo-nuclear scattering is encoded in an elastic nuclear form factor $G_{2}^{\mathrm{el}}\left[q^{2}\right]$ (see Eq. (5.22) below). This form factor plays an important role of suppressing scattering at low $-q^{2}$, that is, at scales larger than the $\mathrm{Si}$ atom. 


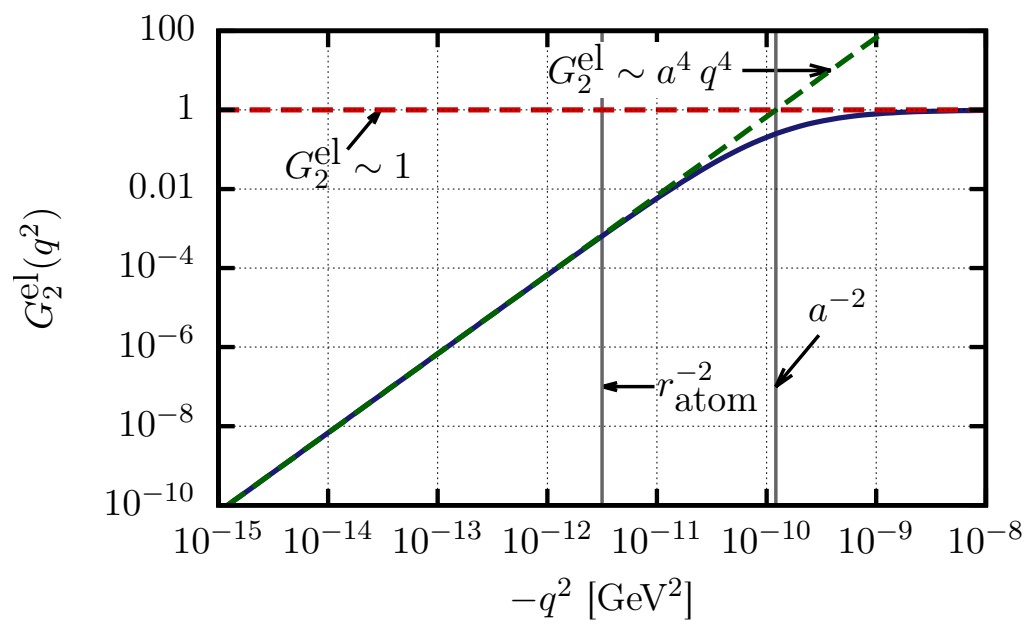

Figure 5.5: The elastic form factor $G_{2}^{\mathrm{el}}\left(q^{2}\right)$. The dashed lines show the limiting behavior $G_{2}^{\text {eq }} \sim a^{4} q^{4}$ for $\left|a^{2} q^{2}\right| \ll 1$ (green dashed line) and $G_{2}^{\text {el }} \sim 1$ for $\left|a^{2} q^{2}\right| \gg 1$ (red dashed line). The scale at which screening of the nucleus becomes important is denoted by $a$, which is smaller than the $\mathrm{Si}$ atomic radius, $r_{\text {atom }}$. At scales well outside the atom, corresponding to small $-q^{2}$, nuclear conversion is suppressed by the form factor screening.

Let us discuss briefly the behavior of the nuclear form factor. The threshold for an $E_{\gamma} \simeq 60 \mathrm{GeV}$ photon to convert to an $e^{+} e^{-}$pair is at $-q^{2}=4 m^{4} / E_{\gamma}^{2} \simeq 10^{-18} \mathrm{GeV}^{2}$, with $m$ the electronic mass, but can occur at momentum transfers as large as $-q^{2} \sim 10^{-6}$ $\mathrm{GeV}^{2}$. This should be compared with the radius of the $\mathrm{Si}$ atom, $r_{\text {atom }} \simeq 1.1 \AA$ [279] or $r_{\text {atom }}^{-2} \simeq 10^{-12} \mathrm{GeV}^{2}$, and with the nuclear radius $r_{\text {nuclear }} \simeq 4 \mathrm{fm}$ which gives $r_{\text {nucl }}^{-2} \simeq$ $10^{-3} \mathrm{GeV}^{2}$. Within the $-q^{2}$ range of interest for conversion $-10^{-18}$ up to $10^{-6} \mathrm{GeV}^{2}-$ the nuclear charge is thus screened at low $-q^{2}$ by the atomic shell electrons. In this work we use the simple expression for the atomic form factor [280] described in detail in [272] and given by

$$
G_{2}^{\mathrm{el}}\left(q^{2}\right)=\frac{a^{4} q^{4}}{\left(1-a^{2} q^{2}\right)^{2}},
$$

where $a=184.15(2.718)^{-1 / 2} Z^{-1 / 3} / m$ and $Z$ is the atomic number of the nucleus. For ${ }^{28} \mathrm{Si}, a^{-2}=1.22 \times 10^{-10} \mathrm{GeV}^{2}$. There are two limits of interest. The first is $\left|a^{2} q^{2}\right| \gg 1$ in which $G_{2}^{\mathrm{el}} \sim 1$, the second is the limit $\left|a^{2} q^{2}\right| \ll 1$ in which $G_{2}^{\mathrm{el}} \sim q^{4}$, see Fig. 5.5. That is, the form factor suppresses the $\mathrm{BH}$ cross-section for small $-q^{2} \ll a^{2}$. To the 
extent that the $1 / q^{4}$ factor in the $\mathrm{BH}$ cross-section determines the dominant phase space configurations of the final states, this suppression significantly alters the important regions of phase space for $\mathrm{BH}$ conversion up to the $a^{-2}$ scale. Specifically, the form factor increases the probability of significantly acoplanar BH conversions.

\subsection{The Higgs-Bethe-Heitler process}

In this section we present a formal analysis of the Higgs-Bethe-Heitler $(\mathrm{HBH})$ process, $h \rightarrow \gamma \gamma$ with both photons converting to $e^{+} e^{-}$pairs. The main result is that we obtain compact, leading order expressions for the $\mathrm{HBH}$ rate, and gain insight into the structure of the terms sensitive to $\mathrm{CP}$ violation.

\subsubsection{Amplitude and cross-section}

The amplitude of interest is given by a menorah diagram, consisting of a $h \rightarrow \gamma \gamma$ and two BH conversion subdiagrams, summed over internal photon polarizations, viz.

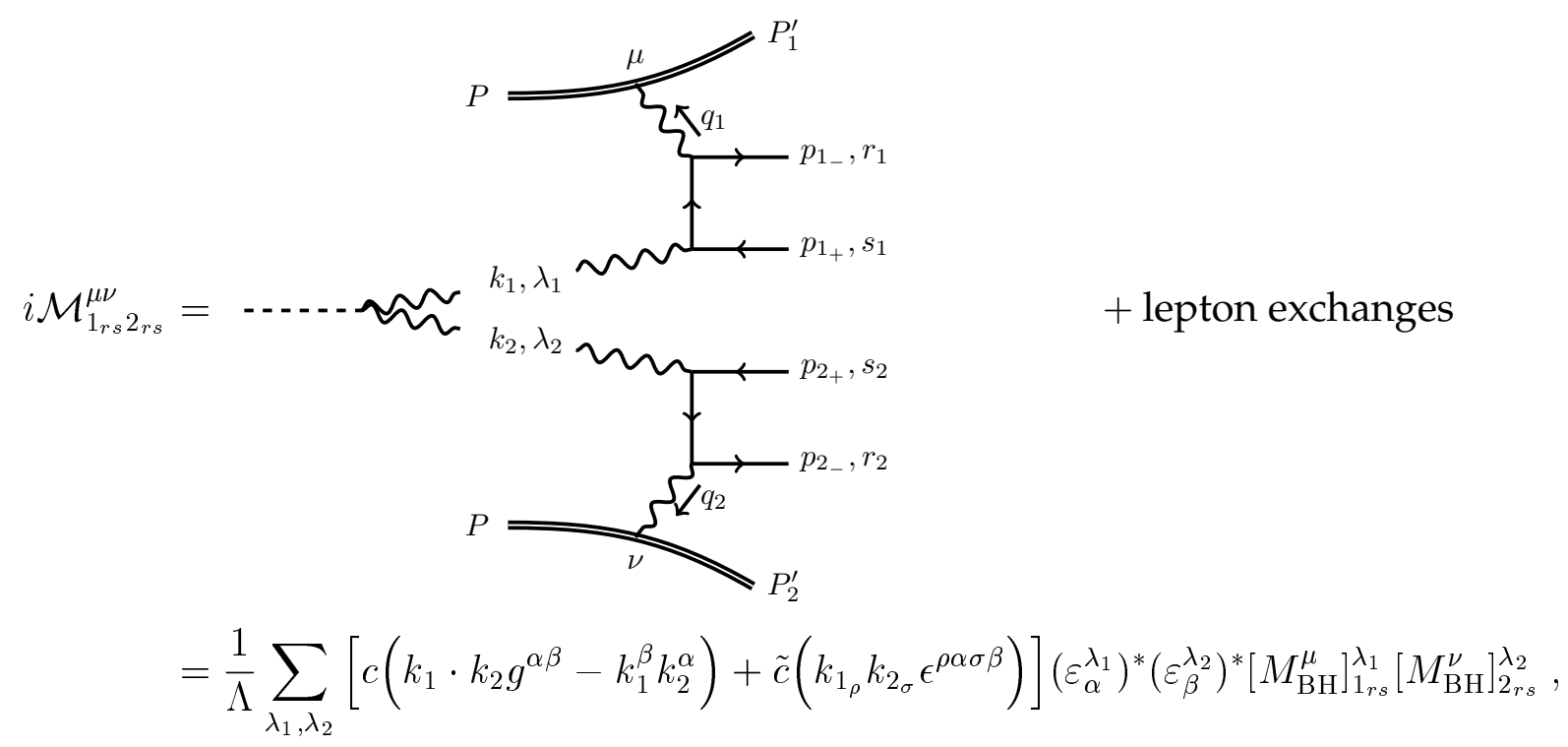


in which $\Lambda=\pi v / e^{6} \alpha$, for QED coupling $e$. The $\mathrm{BH}$ amplitudes are

$$
\left[M_{\mathrm{BH}}^{\mu}\right]_{i_{r s}}^{\lambda_{i}}=\bar{u}^{r_{i}}\left(p_{i_{-}}\right)\left[\not^{\lambda_{i}} \frac{i}{\not p_{i_{-}}-\not k_{i}-m} \gamma^{\mu}+\gamma^{\mu} \frac{i}{\not k_{i}-\not p_{i_{+}}-m} \phi^{\lambda_{i}}\right] v^{s_{i}}\left(p_{i_{+}}\right),
$$

where we have not yet taken nuclear form factors into account, and kept explicit the corresponding Lorentz index of the nuclear electromagnetic current. The Latin subscripts, $i=1,2$, label each photon, while $s_{i}, r_{i}=1,2$ are respectively the positron and electron spins (see Eq. (C.8)), and $\lambda_{i}= \pm$ the outgoing photon helicities from the $h \rightarrow \gamma \gamma$ vertex. We have also suppressed repetitions of the photon index, such that $X_{i_{r s}}$ is henceforth a shorthand for $X_{i_{r_{i}} s_{i}}$. We shall often refer to the BH subdiagrams for each photon as the photon branch.

We assume both nuclei are initially at rest in the lab frame, so $P^{\mu}=(M,(m) 0)$ where $M$ is the mass of the nucleus. The Higgs need not be at rest in the lab frame. As discussed in Sec. 5.3.1, we assume quasi-elastic scattering, that is $P_{i}^{\prime 2}=P^{2}=M^{2}$. This implies that $2 q_{i} \cdot P+q_{i}^{2}=0$, and so

$$
E_{i}-E_{i_{+}}-E_{i_{-}}+q_{i}^{2} / 2 M=0
$$

where $E_{i}, E_{i_{ \pm}}$are the photon and lepton energies respectively. It is also convenient to define

$$
Q_{i}^{\mu} \equiv \frac{1}{M}\left(P^{\mu}-q_{i}^{\mu} \frac{q_{i} \cdot P}{q_{i}^{2}}\right)=\frac{1}{M}\left(P^{\mu}+\frac{q_{i}^{\mu}}{2}\right),
$$

under quasi-scattering conditions. The exchange energy with the nucleus $q_{i}^{0} \equiv P_{i}^{\prime 0}-$ $P_{i}^{0} \ll M$. I.e. the nucleus velocity is non-relativistic, so to an excellent approximation, it follows that

$$
E_{i} \simeq E_{i_{+}}+E_{i_{-}}, \quad Q_{i}^{\mu} \simeq(1,(m) 0)
$$


in the lab frame.

We define the BH nuclear form factor tensors on each photon branch [272],

$$
\mathcal{W}_{i}^{\mu \nu}=-W_{1}\left(q_{i}^{2}\right)\left(g^{\mu \nu}-\frac{q_{i}^{\mu} q_{i}^{\nu}}{q_{i}^{2}}\right)+W_{2}\left(q_{i}^{2}\right) Q_{i}^{\mu} Q_{i}^{\nu}
$$

such that the unpolarized $\mathrm{HBH}$ squared amplitude

$$
|\mathcal{M}|^{2} \prod_{i=1,2} 2 M \delta\left(M^{2}-P_{i}^{\prime 2}\right)=\frac{1}{q_{1}^{4} q_{2}^{4}} \sum_{r_{i}, s_{i}} \mathcal{M}_{1_{r s} 2 r s}^{\mu \nu} \mathcal{M}_{1_{r s} 2_{r s}}^{* \mu^{\prime} \nu^{\prime}} \mathcal{W}_{1 \mu \mu^{\prime}} \mathcal{W}_{2 \nu \nu^{\prime}}
$$

On the left of Eq. (5.21) we have factored out the $\delta$-functions that enforce quasi-elastic scattering. The form factor $W_{1}\left(q^{2}\right)=0$ for quasi-elastic scattering on a spin-0 nuclear target [276], while

$$
W_{2}\left(q^{2}\right)=2 M \delta\left(M^{2}-P^{\prime 2}\right) G_{2}^{\mathrm{el}}\left(q^{2}\right),
$$

in which $G_{2}^{\mathrm{el}}\left(q^{2}\right)$ is the form factor given in Eq. (5.14). Hence the unpolarized $\mathrm{HBH}$ squared amplitude reduces to

$$
|\mathcal{M}|^{2}=\frac{G_{2}^{\mathrm{el}}\left(q_{1}^{2}\right) G_{2}^{\mathrm{el}}\left(q_{2}^{2}\right)}{q_{1}^{4} q_{2}^{4}} \sum_{r_{i}, s_{i}} \mathcal{M}_{1_{r s} 2 r s}^{\mu \nu} \mathcal{M}_{1_{r s} 2_{r s}}^{* \mu^{\prime}{ }^{\prime}} Q_{1_{\mu}} Q_{\mu_{\mu^{\prime}}} Q_{2 \nu} Q_{2 \nu^{\prime}}
$$

\subsubsection{Helicity structure}

One may calculate $|\mathcal{M}|^{2}$ via the usual Feynman method, which relies on polarization completeness relations to compute traces. This approach leads to thousands of terms, of which the naïvely dominant terms cancel due to Ward identities. Extracting leading order expressions is therefore difficult, and moreover, high numerical precision is required for numerical stability. As an alternative, we employ a spin and helicity analysis combined with the spinor-helicity formalism to compute the $\mathrm{HBH}$ amplitudes. These may be subsequently squared and summed over external spins to produce the 
full $\mathrm{HBH}$ rate. In the following we provide a brief overview of these results, while details are provided in Appendices C.I and C.II.

The BH spin-helicity amplitudes are defined as

$$
\alpha_{i_{r s}}^{ \pm} \equiv\left[M_{\mathrm{BH}}^{\mu}\right]_{i_{r s}}^{ \pm} Q_{i_{\mu}} / q_{i}^{2} .
$$

With reference to Eqs. (5.15) and (5.23), the HBH spin-helicity amplitudes are correspondingly

$$
i \mathcal{M}_{1_{r s} 2_{r s}}^{\lambda_{1} \lambda_{2}}=\frac{\sqrt{\mathcal{G}_{1} \mathcal{G}_{2}}}{\Lambda}\left[c\left(k_{1} \cdot k_{2} g^{\alpha \beta}-k_{1}^{\beta} k_{2}^{\alpha}\right)+\tilde{c}\left(k_{1_{\rho}} k_{2_{\sigma}} \epsilon^{\rho \alpha \sigma \beta}\right)\right]\left(\varepsilon_{\alpha}^{\lambda_{1}}\right)^{*}\left(\varepsilon_{\beta}^{\lambda_{2}}\right)^{*} \alpha_{1_{r s}}^{\lambda_{1}} \alpha_{2_{r s}}^{\lambda_{2}},
$$

where we introduced the abbreviation

$$
\mathcal{G}_{i} \equiv G_{2}^{\mathrm{el}}\left(q_{i}^{2}\right)
$$

Making use of the spinor-helicity formalism (see Appendix C.I), we obtain the spin amplitudes

$$
\mathcal{M}_{1_{r s} 2 r s} \equiv \sum_{\lambda_{1} \lambda_{2}} \mathcal{M}_{1_{r s} 2_{r s}}^{\lambda_{1} \lambda_{2}}=\frac{m_{h}^{2}}{\Lambda} \sqrt{\mathcal{G}_{1} \mathcal{G}_{2}} \sqrt{c^{2}+\tilde{c}^{2}}\left(e^{i \xi} \alpha_{1_{r s}}^{+} \alpha_{2_{r s}}^{+}+e^{-i \xi} \alpha_{1_{r s}}^{-} \alpha_{2_{r s}}^{-}\right)
$$

These are reminiscent of equation (5.9) with final leptonic spin states $\left\langle r_{i} s_{i}\right|$ and $\alpha_{i_{r s}}^{ \pm} \propto$ $\left\langle s_{i} r_{i} \mid \pm\right\rangle$, as expected. The HBH square-amplitude is correspondingly

$$
|\mathcal{M}|^{2} \equiv \sum_{1_{r s} 2_{r s}}\left|\sum_{\lambda_{1} \lambda_{2}} \mathcal{M}_{1_{r s} 2_{r s}}^{\lambda_{1} \lambda_{2}}\right|^{2}=m_{h}^{4} \mathcal{G}_{1} \mathcal{G}_{2} \frac{c^{2}+\tilde{c}^{2}}{4 \Lambda^{2}} \sum_{1_{r s} 2 r s}\left|\alpha_{1_{r s}}^{+} \alpha_{2_{r s}}^{+} e^{i \xi}+\alpha_{1_{r s}}^{-} \alpha_{2_{r s}}^{-} e^{-i \xi}\right|^{2}
$$

Note that the sum over photon polarizations is inside the absolute value as expected for entangled $h \rightarrow 2 \gamma \rightarrow 2\left(e^{+} e^{-}\right)$decay, but the sum over lepton spins is incoherent. Eq. (5.28) shows that the BH spin-helicity amplitudes $\alpha_{i_{r s}}^{ \pm}$are all one needs to deter- 
mine the entire $\mathrm{HBH}$ square amplitude. Parity invariance of the $\mathrm{BH}$ amplitudes relates amplitudes of opposite helicity and spins to their complex conjugates,

$$
\left(\alpha_{r s}^{+}\right)^{*}=\eta_{r s} \alpha_{\bar{r} \bar{s}}^{-},
$$

where $\bar{s}$ is the opposite spin to $s$, and $\eta_{r s}^{2}=1, \eta_{r s}=\eta_{\bar{r} \bar{s}}$. Hence we need only determine $\alpha_{i_{r s}}^{+}$. We shall see below that spinor-helicity methods, when applied to $\alpha_{i_{r s}}^{ \pm}$also allow for a well-controlled expansion of dominant, sub-dominant and negligible terms in $|\mathcal{M}|^{2}$.

We see explicitly from Eq. (5.28) that the helicity interference terms are equivalent to the $\xi$-dependent terms, as discussed in sections 5.2.2 and 5.2.3. For a particular leptonic spin configuration $\left\{r_{i}, s_{i}\right\}$, helicity interference occurs so long as $\alpha_{i_{r s}}^{+}$and $\alpha_{i_{r s}}^{-}$are both non-zero. However, we see in appendix C.II, and in particular in Eq. (C.13), that for our particular choice of spinor basis (see Eq. (C.8))

$$
\left|\alpha_{i_{11}}^{-}\right| \sim\left|\alpha_{i_{12}}^{-}\right| \sim\left|\alpha_{i_{21}}^{-}\right| \gg\left|\alpha_{i_{22}}^{-}\right|
$$

or equivalently $\left|\alpha_{i_{22}}^{+}\right| \sim\left|\alpha_{i_{12}}^{+}\right| \sim\left|\alpha_{i_{21}}^{+}\right| \gg\left|\alpha_{i_{11}}^{+}\right|$. This hierarchy means that we may therefore discard any terms containing either $\alpha_{i_{22}}^{+}$or $\alpha_{i_{11}}^{-}$as subleading. It follows that the leading order squared amplitude is

$$
|\mathcal{M}|^{2} \simeq m_{h}^{4} \frac{c^{2}+\tilde{c}^{2}}{4 \Lambda^{2}} \mathcal{G}_{1} \mathcal{G}_{2}\left\{2\left|\alpha_{1_{11}}^{-} \alpha_{2_{11}}^{-}\right|^{2}+2 \sum_{\substack{j \neq k \\ r_{k} \neq s_{k}}}\left|\alpha_{j_{11}}^{-} \alpha_{k_{r s}}^{-}\right|^{2}+\sum_{\substack{r_{1} \neq s_{1} \\ r_{2} \neq s_{2}}}\left|\alpha_{1_{r s}}^{+} \alpha_{2_{r s}}^{+} e^{i \xi}+\alpha_{1_{r s}}^{-} \alpha_{2_{r s}}^{-} e^{-i \xi}\right|^{2}\right\}
$$

while the leading interference term is

$$
\left.|\mathcal{M}|^{2}\right|_{\text {int }} \simeq 2 m_{h}^{4} \frac{c^{2}+\tilde{c}^{2}}{\Lambda^{2}} \mathcal{G}_{1} \mathcal{G}_{2} \operatorname{Re}\left\{\alpha_{1_{12}}^{-} \alpha_{1_{21}}^{-} \alpha_{2_{12}}^{-} \alpha_{2_{21}}^{-} e^{-2 i \xi}\right\}
$$


We see that only $\alpha_{12,21}^{-}$(or equivalently $\alpha_{21,12}^{+}$) amplitudes enter the leading order $\xi$ dependent interference terms.

The CP odd helicity interference term in (5.32) is proportional to $c \tilde{c}$,

$$
\left.|\mathcal{M}|^{2}\right|_{\text {int }, \mathrm{CP}-\text { odd }}=-2 m_{h}^{4} \frac{c \tilde{c}}{\Lambda^{2}} \mathcal{G}_{1} \mathcal{G}_{2} \operatorname{Im}\left\{\alpha_{1_{12}}^{-} \alpha_{1_{21}}^{-} \alpha_{2_{12}}^{-} \alpha_{2_{21}}^{-}\right\}
$$

Note that interference terms between amplitudes, produced by the $F F$ and $F \tilde{F}$ operators respectively, are $\mathrm{CP}$ odd. However, the helicity amplitudes under consideration here receive contributions from both $\mathrm{CP}$ odd and $\mathrm{CP}$ even operators - manifestly they depend on $\xi$ - and hence helicity interference terms contain both CP-even and CP-odd pieces. Consequently, we interpret the remaining piece of the helicity interference term to be the CP-even piece,

$$
\left.|\mathcal{M}|^{2}\right|_{\text {int }, \mathrm{CP}-\text { even }}=m_{h}^{4} \frac{c^{2}-\tilde{c}^{2}}{\Lambda^{2}} \mathcal{G}_{1} \mathcal{G}_{2} \operatorname{Re}\left\{\alpha_{1_{12}}^{-} \alpha_{1_{21}}^{-} \alpha_{2_{12}}^{-} \alpha_{2_{21}}^{-}\right\}
$$

This term has quadratic dependence on $\tilde{c}^{2}$, albeit a different one than the total rate (5.12).

\subsubsection{The Bethe-Heitler helicity amplitudes}

In Eqs. (5.31) and (5.32) we have expressed the leading order HBH rate and interference term in terms of individual $\mathrm{BH}$ helicity amplitudes, $\alpha_{r s}^{ \pm}$. In this subsection we proceed to present the leading order terms of these amplitudes in a readily accessible notation, for the special case that the Higgs is at rest in the lab frame. The results below are achieved via spinor-helicity techniques; a more comprehensive presentation of the derivation of these results is provided in Appendix C.II. There, explicit results for each spin helicity amplitude $\alpha_{i_{r s}}^{ \pm}$are collected in Eqs. (C.12), while the leading order results, obtained by power counting in $m / m_{h}$, are provided in (C.13). The compact results be- 
low will permit us, in the next section of this chapter, to study the encoding of the CPV structure in the $\mathrm{HBH}$ rate at an analytic level.

Before proceeding, we may first derive a new result concerning the well-studied unpolarized BH square amplitude, $\left|\mathcal{M}_{\mathrm{BH}_{i}}\right|^{2}=\sum_{r s \lambda}\left|\alpha_{i_{r s}}^{\lambda}\right|^{2}$. Using Eqs. (C.12) one may show that

$$
\left|\mathcal{M}_{\mathrm{BH}_{i}}\right|^{2} \simeq 8 \frac{\mathcal{G}_{i} m^{2}}{q_{i}^{4}}\left[\frac{E_{i_{-}}\left(k_{i} \cdot p_{i_{-}}\right)-E_{i_{+}}\left(k_{i} \cdot p_{i_{+}}\right)}{\left(k_{i} \cdot p_{i_{-}}\right)\left(k_{i} \cdot p_{i_{+}}\right)}\right]^{2}-4 \frac{\mathcal{G}_{i}}{q_{i}^{2}} \frac{E_{i_{+}}^{2}+E_{i_{-}}^{2}}{\left(k_{i} \cdot p_{i_{-}}\right)\left(k_{i} \cdot p_{i_{+}}\right)}
$$

This compact expression for the $\mathrm{BH}$ rate in the quasi-elastic scattering limit is novel to this work. A numerical analysis and validation of the resulting $\mathrm{BH}$ differential crosssection is provided in Appendix C.IV.

Now, in the special case that the Higgs is at rest in the lab frame, that is $(m) P_{h}=$ $(m) 0$, Eqs. (C.13) for the $\alpha_{i_{r s}}^{ \pm}$collapse to very simple expressions. Using the leading order results (C.13) and assuming $\theta_{ \pm} \ll 1$, for each branch one finds

$$
\begin{aligned}
& \alpha_{11}^{-}=-\left(\alpha_{22}^{+}\right)^{*} \simeq \frac{2 \sqrt{2 \gamma_{+} \gamma_{-}}}{q^{2}}\left(\frac{1}{1+\gamma_{+}^{2} \theta_{+}^{2}}-\frac{1}{1+\gamma_{-}^{2} \theta_{-}^{2}}\right), \\
& \alpha_{12}^{-}=+\left(\alpha_{21}^{+}\right)^{*} \simeq \pm \frac{2 \sqrt{2 \gamma_{+} \gamma_{-}}}{q^{2}} \frac{\gamma_{\mp}}{\gamma_{+}+\gamma_{-}}\left(\frac{\gamma_{+} \theta_{+} e^{-i \phi_{+}}}{1+\gamma_{+}^{2} \theta_{+}^{2}}+\frac{\gamma_{-} \theta_{-} e^{-i \phi_{-}}}{1+\gamma_{-}^{2} \theta_{-}^{2}}\right), \\
& \alpha_{22}^{-}=-\left(\alpha_{11}^{+}\right)^{*} \simeq 0 .
\end{aligned}
$$

Here $\theta_{ \pm}$and $\phi_{ \pm}$are respectively the polar and azimuthal angles defined with respect to the branch parent photon momentum, as shown in Fig. 5.6, and $\gamma_{ \pm} \equiv E_{ \pm} / m \gg 1$. We also assume that the $\mathcal{O}(\theta)$ terms do not cancel completely. The latter may occur on the phase space slice $\gamma_{+} \theta_{+}=\gamma_{-} \theta_{-}$and $\left|\phi_{-}-\phi_{+}\right|=\pi$, corresponding to minimal $\left|q^{2}\right|$. Excellent numerical validation of this expansion implies that the neighborhood of this phase space slice, on which the expansion fails, is actually of negligibly small measure. 


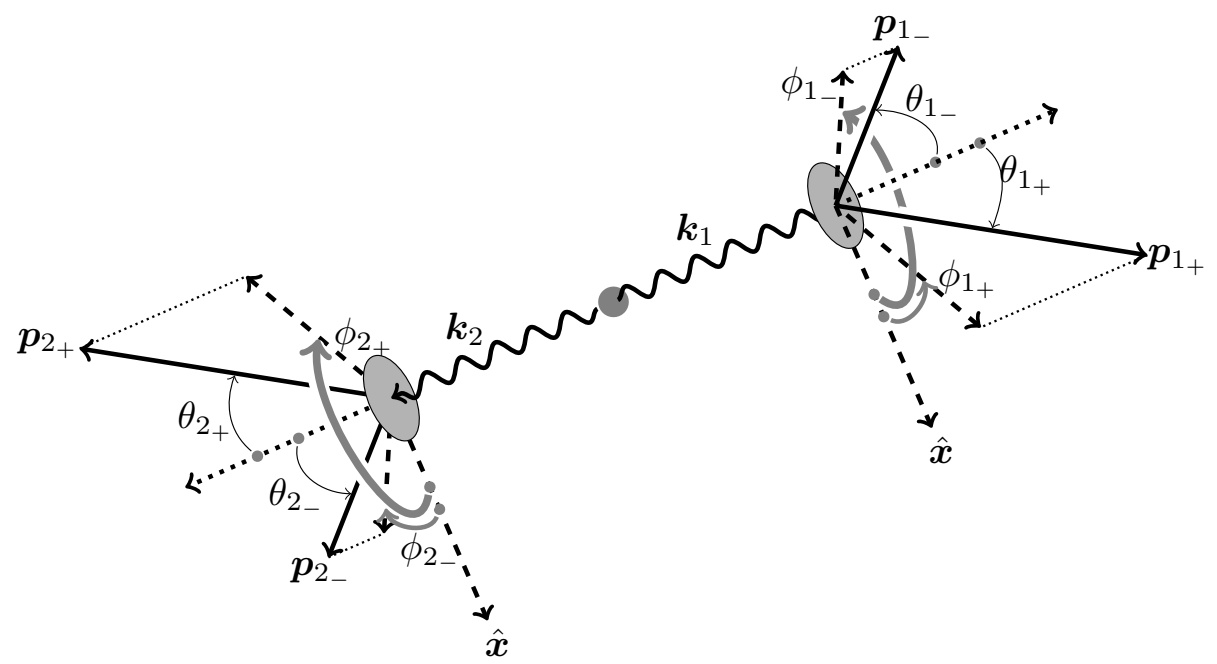

Figure 5.6: Definitions of local spherical polar angles $\left\{\theta_{i_{ \pm}}, \phi_{i_{ \pm}}\right\}$. Note in particular that azimuthal angles, $\phi_{i_{ \pm}}$are positively oriented with respect to their parent photons, and are defined with respect to an azimuth $(\hat{m}) x$ common to both branches. Polar angles are defined with respect to parent photon momentum (black dotted).

Finally, we may approximate $q^{2}$ by

$$
-q^{2} \simeq m^{2}\left(\gamma_{+}^{2} \theta_{+}^{2}+\gamma_{-}^{2} \theta_{-}^{2}+2 \gamma_{-} \gamma_{+} \theta_{-} \theta_{+} \cos \left(\phi_{-}-\phi_{+}\right)\right)+\frac{m^{2}}{4}\left[\frac{1}{\gamma_{+}}+\frac{1}{\gamma_{-}}\right]^{2} .
$$

Eqs. (5.36) and (5.37), when combined with Eq. (5.28), form one of the central results of this chapter: a compact form of the HBH rate, which is both numerically stable, and whose structure may now be studied analytically. For example, it is now manifest that $\mathrm{HBH}$ polar angular structure is dominantly controlled just by the $k \cdot p_{+} / m^{2} \simeq 1+\gamma_{ \pm} \theta_{ \pm}$ denominators, which produce a peak near $\theta_{ \pm} \gamma_{ \pm} \sim 1$. More importantly, we see that the $\alpha_{12,21}^{ \pm}$amplitudes, that control the helicity interference terms, contain phases which are the leptonic azimuthal orientations, $\phi_{i_{ \pm}}$. This non-trivial result, when combined with Eq. (5.32) shows us that, at leading order, $\xi$ manifests as a phase shift in the relative azimuthal orientations of leptons with different parent photons. 


\subsection{Sensitivity to CPV}

In this section we assess the potential sensitivity to $\mathrm{CP}$ violation. To do this, we consider a number of CPV sensitive observables and propose several sets of kinematic cuts that can enhance the CPV signal. These sets of cuts require that the lepton-lepton opening angle (5.13), $\theta_{\ell \ell}$, can be resolved, as well as the two photon-lepton angles, $\theta_{ \pm}$. In the following we mostly consider angular resolution cuts of the form

$$
\theta_{\ell \ell}, \theta_{ \pm}>\theta_{\text {cut }}
$$

Following Sec. 5.3.2, we apply an angular resolution cut $\theta_{\text {cut }}=10^{-4}$, which is at the edge of what may be possible with present detectors, and a looser, futuristic $\theta_{\text {cut }}=$ $10^{-5}$, intended to show that very large CPV effects are possible in principle.

We emphasize that as our measurement is a novel, challenging one, our goal here is not to conduct a full collider analysis including backgrounds. Rather our aim is to identify the types of observables that can probe CP violation in $h \rightarrow \gamma \gamma$, and estimate how well they do under ideal circumstances: a high efficiency in reconstructing conversions and a signal rich channel. This is in the anticipation that such circumstances might materialize in a future LHC running or at a Higgs factory. 


\subsubsection{Differential scattering rate}

The HBH differential scattering rate for the full $3 \rightarrow 6$ process (Higgs plus two nuclei to two nuclei and two $e^{+} e^{-}$pairs) in the lab frame is

$$
\begin{aligned}
d \Gamma & \propto|\mathcal{M}|^{2} d \Pi_{h \rightarrow \gamma \gamma} d \Pi_{\mathrm{BH}_{1}} d \Pi_{\mathrm{BH}_{2}} \\
& \propto \frac{1}{(2 \pi)^{12}}|\mathcal{M}|^{2}\left[\prod_{\substack{i=1,2 \\
\alpha= \pm}} \frac{d^{3}(m) p_{i_{\alpha}}}{2 E_{i}}\right]\left[\prod_{i=1,2} \frac{d^{3}(m) P_{i}^{\prime}}{2 E_{i}^{\prime}} \frac{d^{3}(m) k_{i}}{2 E_{i_{\alpha}}} \delta^{(4)}\left(q_{i}+p_{i_{+}}+p_{i_{-}}-k_{i}\right)\right] \delta^{(4)}\left(P_{h}-k_{1}-k_{2}\right),
\end{aligned}
$$

with $|\mathcal{M}|^{2}$ given by Eq. (5.28). Integrating over the out-going nuclear momenta and all other momenta in delta functions, we obtain in the limit $|q| \ll M$

$$
d \Gamma \propto \frac{1}{(2 \pi)^{12}} \frac{m_{h}^{2}}{4 M^{2}}|\mathcal{M}|^{2}\left[\prod_{\substack{i=1,2 \\ \alpha= \pm}}\left|(m) p_{i_{\alpha}}\right| d \Omega_{i_{\alpha}}\right] d E_{1_{-}} d E_{2-} d \Omega_{1},
$$

with $d \Omega_{i_{\alpha}}$ the solid angle for each lepton momentum $(m) p_{i_{\alpha}}$, and $d \Omega_{1}$ the solid angle for photon ' 1 '. The photon labels are extrinsic. We take photon ' 1 ', say, as westwards going (if Higgs is not at rest one can also take it to be, e.g., the more energetic photon).

For simplicity, we assume henceforth that the Higgs is at rest in the lab frame, that is $P_{h}=\left(m_{h},(m) 0\right)$. In the Higgs rest frame the photon angular dependence is isotropic, and the integration over $d \Omega_{1}$ is trivial. Dropping the prefactors, the differential scattering rate becomes

$$
d \Gamma \propto|\mathcal{M}|^{2}\left[\prod_{\substack{i=1,2 \\ \alpha= \pm}}\left|(m) p_{i_{\alpha}}\right| d \Omega_{i_{\alpha}}\right] d E_{1_{-}} d E_{2_{-}} .
$$

We may now proceed to consider CPV observables. 


\subsubsection{Global sensitivity to $\mathrm{CP}$ violation}

In principle all the information about $\tilde{c} \neq 0$ (or equivalently $\xi \neq 0$ ) is encoded in the full HBH differential distribution. The coefficient $\tilde{c}$ may be determined by a matrix element method [281-286], as long as backgrounds can be kept under control. Estimating the full power of the matrix element method is beyond the scope of this work.

To test the sensitivity of $\mathrm{HBH}$ to $\tilde{c}$ we instead introduce a parameter

$$
Z_{B}(c, \tilde{c})=\frac{\int|d \Gamma(\mathrm{SM}) / d \mathrm{PS}-d \Gamma(c, \tilde{c}) / d \mathrm{PS}| d \mathrm{PS}}{\Gamma(\mathrm{SM})} .
$$

The parameter $Z_{B}$ can be thought of as a proxy for the sensitivity of the matrix element method, once one integrates over the full phase space. In the top panel of Fig. 5.7 we show the value of $Z_{B}$ in the $(c, \tilde{c})$ plane. There we see that the deviation from the SM is mostly due to the $c^{2}+\tilde{c}^{2}$ enhancement of the $h \rightarrow \gamma \gamma$ rate, which need not arise from $\mathrm{CP}$ violation. Such an enhancement is best detected by measuring the overall $h \rightarrow \gamma \gamma$ rate, and not using our method.

To assess the sensitivity to $\mathrm{CP}$ violation alone, we should restrict our attention to the circular contour $c^{2}+\tilde{c}^{2}=c_{\mathrm{SM}}^{2}$, on which the total $\mathrm{HBH}$ rate matches the $\mathrm{SM}$ rate for any $\xi$. This contour is shown as a white circle in the top panel of Fig. 5.7. To this end, we define a second quantity

$$
\left.Z_{B}^{\mathrm{c}}(\xi) \equiv Z_{B}(\xi)\right|_{c^{2}+\tilde{c}^{2}=c_{\mathrm{SM}}^{2}}=\frac{\int|d \hat{\Gamma}(0) / d \mathrm{PS}-d \hat{\Gamma}(\xi) / d \mathrm{PS}| d \mathrm{PS}}{\hat{\Gamma}(0)}
$$

where $d \hat{\Gamma}(\xi)$ is the differential $\mathrm{HBH}$ rate with the enhancement of the total $h \rightarrow \gamma \gamma$ rate factored out,

$$
d \Gamma(c, \tilde{c}) / d \mathrm{PS}=\frac{\left(c^{2}+\tilde{c}^{2}\right)}{c_{\mathrm{SM}}^{2}} d \hat{\Gamma}(\xi) / d \mathrm{PS} .
$$

Note that in general $Z_{B} \in[0, \infty)$, but $Z_{B}^{\mathrm{c}} \in[0,2]$. 

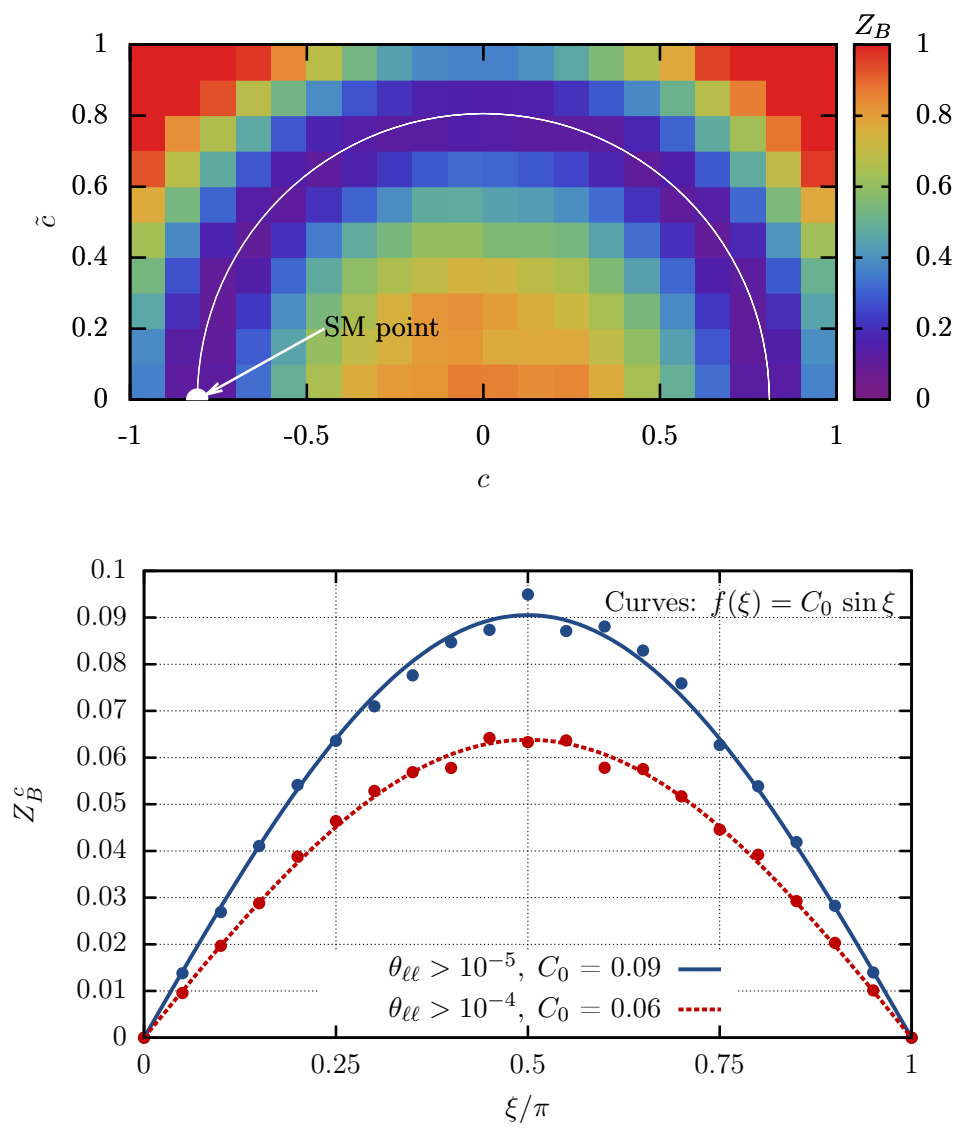

Figure 5.7: Top panel: $Z_{B}$ in the $c-\tilde{c}$ plane, with the SM point at $(c, \tilde{c})=(-0.81,0)$. Bottom panel: $Z_{B}^{c}$ as a function of $\xi=\tan ^{-1}(\tilde{c} / c)$. The scatter of the data points is a numerical artifact.

The bottom panel in Fig. 5.7 shows the value of $Z_{B}^{\mathrm{c}}$ as a function of $\xi$. The sinusoidal dependence of $Z_{B}^{\mathrm{c}}$ on $\xi$ is not unexpected. For instance, in the $h \rightarrow \gamma \gamma$ toy example we considered in Sec. 5.2.3, in which the angle between linear polarizations is measured, one has from Eq. (5.12)

$$
\left.Z_{B}^{\mathrm{c}}(\xi)\right|_{h \rightarrow \gamma \gamma}=\frac{1}{\pi} \int_{0}^{2 \pi}\left|\cos ^{2}(\phi+\xi)-\cos ^{2}(\phi)\right| d \phi=\frac{4}{\pi}|\sin \xi| .
$$

By comparing this toy system with $\mathrm{HBH}$, it is therefore natural to deduce that $Z_{B}^{\mathrm{c}}(\xi) /|\sin \xi|$ provides a measure of the average $\mathrm{CPV}$ signal size obtainable via the matrix element 
method, for any $c, \tilde{c}$. That is, this measure is independent of $\xi$ and the overall normalization $c^{2}+\tilde{c}^{2}$. The fit in Fig. 5.7 suggests this CPV signal is $\mathcal{O}(10 \%)$ for an angular resolution cut $\theta_{\ell \ell}>10^{-5}$, and $\mathcal{O}(5 \%)$ for $\theta_{\ell \ell}>10^{-4}$. Hence, for the most pessimistic case that there is no deviation from the SM in the total $h \rightarrow \gamma \gamma$ rate the detection of NP from the full matrix element method will be challenging, even if there is large CPV component in $h \rightarrow \gamma \gamma$.

\subsubsection{Differential azimuthal scattering rate}

Let us also consider the sensitivity of an experiment in which just one relative azimuthal angle - the difference of the azimuthal angles between two opposite branch leptons - is reconstructed, such as the experiment described in Fig. 5.1. From Eqs. (5.28) and (5.36) we saw that $\xi$ manifests as a phase shift in the relative azimuthal orientation of leptons on different branches. It is instructive to write down this manifestation explicitly. Let us transform from the azimuthal coordinates $\phi_{i_{ \pm}}$to the coordinates

$$
\varphi \equiv \phi_{1_{+}}+\phi_{2_{+}}, \quad \varepsilon_{i} \equiv \phi_{i_{-}}-\phi_{i_{+}},
$$

and choose $\phi_{1_{+}}=0$, without loss of generality. With this choice $\varphi$ and $\varepsilon_{1} \in[0,2 \pi)$, while $\varepsilon_{2} \in(-2 \pi, 2 \pi)$. For the case that the Higgs is at rest, we find from Eqs. (5.31) and (5.36) that the $\mathrm{HBH}$ square amplitude, and thus the leading order differential scattering rate, takes the generic form

$$
d \Gamma / d \mathrm{PS}=\frac{1}{q_{1}^{4} q_{2}^{4}}\left[a+\sum_{j} b_{j} \cos \varepsilon_{j}+\sum_{k} c_{k} \cos \left(n_{k} \varepsilon_{1}+m_{k} \varepsilon_{2}+2 \varphi+2 \xi\right)\right],
$$

where $a, b_{j}$ and $c_{k}$ are real functions of $\gamma_{i_{ \pm}}$and $\theta_{i_{ \pm}}$- they span the energy, polar angle phase space, denoted hereafter by $\mathrm{PS}_{\gamma, \theta}$ - but are independent of the azimuthal structure, and $n_{k}, m_{k}$ are positive integers that satisfy $n_{k}+m_{k}=0,1$, or 2. From Eq. (5.37), 
one sees that the $1 / q^{4}$ factors depend on both the azimuthal and polar angles, but in a way such that $q^{4}=\eta(1+\zeta \cos \varepsilon)^{2}$, where $\zeta<1$. Hence $1 / q^{4}$ may be expanded in a power series of $\zeta \cos \varepsilon<1$. Integrating over the $\varepsilon$ acoplanarity angles, one may then show that, with respect to the azimuthal structure, only constant or $\cos (2 \varphi+2 \xi)$ terms survive. That is, the leading order differential scattering rate

$$
\frac{d \Gamma}{d \varphi d \mathrm{PS}_{\gamma, \theta}}=\left(c^{2}+\tilde{c}^{2}\right)\left[\mathcal{A}_{\gamma, \theta}+\mathcal{B}_{\gamma, \theta} \cos (2 \varphi+2 \xi)\right],
$$

in which the $\gamma, \theta$ subscript denotes exclusive dependence on the energy polar angle phase space, $\mathrm{PS}_{\gamma, \theta}$.

The results (5.47) or (5.48) show that $\xi$-dependence and $\gamma, \theta$-dependence factorize. Hence $\xi$ appears only in the azimuthal structure as a phase shift. Specifically, the CPV parameter $\xi$ manifests itself in the inter-branch azimuthal structure in the differential rate

$$
\frac{d \Gamma}{d \varphi}=\left(c^{2}+\tilde{c}^{2}\right)\left[\left\langle\mathcal{A}_{\gamma, \theta}\right\rangle_{\mathrm{PS}_{\gamma, \theta}}+\left\langle\mathcal{B}_{\gamma, \theta}\right\rangle_{\mathrm{PS}_{\gamma, \theta}} \cos (2 \varphi+2 \xi)\right],
$$

which we have now shown is oscillatory with respect to $\varphi$ at leading order. Note that we could have chosen $\varphi$ to be any of the four inter-branch angles $\varphi_{1_{\alpha} 2_{\beta}} \equiv \phi_{1_{\alpha}}+\phi_{2_{\beta}}$, where $\alpha, \beta= \pm$, of which three are linearly independent. Results similar to Eq. (5.48) follow with appropriate replacements.

In the right panel of Fig. 5.8 we show the differential distribution $d \Gamma / d \varphi$ for $\mathrm{HBH}$ events (including a loose cut on the polar angles, at $10^{-5}$ ) for two values of $\xi$. It is evident that the oscillation amplitude, $\langle\mathcal{B}\rangle /\langle\mathcal{A}\rangle$, is small - approximately $\sim 2 \%$ - when averaged over all of the phase space $\mathrm{PS}_{\gamma, \theta}$.

The key question we now wish to address is whether such a small oscillation amplitude is because of small, $\mathcal{O}(1 \%)$ oscillations, or whether there is an $\mathcal{O}(1 \%)$ part of the phase space where deviations from the SM are $\mathcal{O}(1)$. In the language of Eq. (5.48), 
this question can be rephrased in a precise manner: Is $\langle\mathcal{B}\rangle_{U} /\langle\mathcal{A}\rangle_{U}$ small for all $U \subset$ PS, or does there exist $U \subset$ PS such that $\langle\mathcal{B}\rangle_{U} /\langle\mathcal{A}\rangle_{U}$ is $\mathcal{O}(1)$. The latter possibility is phenomenologically preferred, since it permits the extraction of an $\mathcal{O}(1) \mathrm{CPV}$ signal on $U$, which would scale better with increasing statistics.

To address this question, let us begin by examining the coplanar limit. In this limit the acoplanarity angles $\varepsilon_{1,2}$ are both zero, and we have from Eq. (5.47)

$$
\frac{d \Gamma}{d \varphi d \mathrm{PS}_{\gamma, \theta}}=\mathcal{A}_{\gamma, \theta}^{\mathrm{co}}+\mathcal{B}_{\gamma, \theta}^{\mathrm{co}} \cos (2 \xi+2 \varphi)
$$

In the coplanar limit the size of the modulation is given by

$$
\frac{\mathcal{B}_{\gamma, \theta}^{\mathrm{co}}}{\mathcal{A}_{\gamma, \theta}^{\mathrm{co}}}=\prod_{i=1,2} \frac{\mathcal{R}_{i}\left(1-\gamma_{i_{+}} \theta_{i_{+}} \gamma_{i_{-}} \theta_{i_{-}}\right)^{2}}{\left(1+\gamma_{i_{+}}^{2} \theta_{i_{+}}^{2}\right)\left(1+\gamma_{i_{-}}^{2} \theta_{i_{-}}^{2}\right)+\mathcal{R}_{i}\left(\gamma_{i_{-}} \theta_{i_{-}}+\gamma_{i_{+}} \theta_{i_{+}}\right)^{2}},
$$

where $\mathcal{R}_{i} \equiv 2 \gamma_{i_{+}} \gamma_{i_{-}} /\left(\gamma_{i_{+}}^{2}+\gamma_{i_{-}}^{2}\right)$. The ratio $\mathcal{B}^{\text {co }} / \mathcal{A}^{\text {co }}$ is small when $\gamma \theta \sim 1$, i.e. near the peak of the square matrix element, but $\mathcal{B}^{\text {co }} / \mathcal{A}^{\text {co }} \rightarrow 1$, for $\gamma \theta \gg 1$ and $\gamma_{ \pm}$not much bigger than $\gamma_{\mp}$. An example is shown in Fig. 5.8, where $E_{i_{ \pm}}$and $\theta_{i_{ \pm}}$are held fixed such that $\theta_{i_{ \pm}} \gg m / E_{i_{ \pm}}$and $\gamma_{+} \sim \gamma_{-}$. In this slice of phase space the azimuthal oscillation amplitude is large and there is a strong sensitivity to CPV. This shows that regions of phase space with large CPV signals exist.

\subsubsection{CPV enhancing cuts}

We now use the results from Sec. 5.4.2 to design kinematic cuts that enhance the sensitivity of $d \Gamma / d \varphi$ to $\mathrm{CP}$ violation. That is, we seek to enhance the ratio $\langle\mathcal{B}\rangle /\langle\mathcal{A}\rangle$ for a particular subset of the $\mathrm{HBH}$ event sample. The cuts we propose fall into two classes: those which are placed on the kinematics of the whole event; and those which are placed independently on individual photons and their daughter leptons. As they use all of the information in the event, including correlations among the two photons, one 

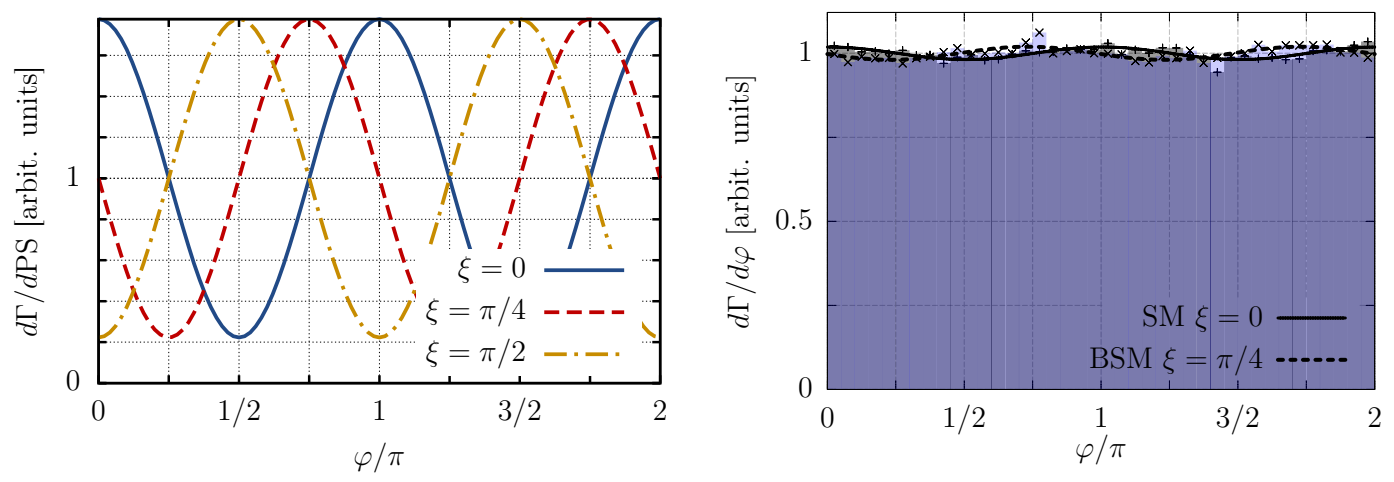

Figure 5.8: Left: Illustration of $\mathcal{O}(1)$ oscillations and phase shifts in the HBH differential rate for a sample coplanar kinematic configuration. The azimuthal angle $\varphi$ in this slice is defined as in Eq. (5.46). The kinematic configuration is: $E_{i_{+}}=E_{i_{-}}=m_{h} / 4, \theta_{i_{+}}=$ $10^{-4}, \theta_{i_{-}}=2 \theta_{i_{+}}$so that $\gamma_{ \pm} \theta_{ \pm} \sim 10 \gg 1$ and $\gamma_{+}=\gamma_{-}$, cf. analysis of Eq. (5.51). Right: The azimuthal distribution $d \Gamma / d \varphi$ for $\xi=0$ and for $\xi=\pi / 4$ with a polar angle cut $\theta_{i_{ \pm}}>10^{-5}$ and $\theta_{\ell \ell}>10^{-5}$. The modulation amplitude is $2 \%$, but will grow to $\mathcal{O}(1)$ once optimization cuts are applied, see Sec. 5.5.4.

might expect that the former produce better CPV signals compared to the latter. As we shall see, however, both classes of cuts perform approximately equally well in enhancing $\langle\mathcal{B}\rangle /\langle\mathcal{A}\rangle$ on their respective phase space subregions.

\subsubsection{Cuts on collective kinematics}

The helicity interference terms (5.32) in the $\mathrm{HBH}$ rate arise dominantly from the term $\operatorname{Re}\left(\alpha_{1_{12}}^{-} \alpha_{1_{21}}^{-} \alpha_{2_{12}}^{-} \alpha_{2_{21}}^{-} e^{-2 i \xi}\right)$. We can use this observation to pick only events in which $\alpha_{1_{12}}^{-} \alpha_{1_{21}}^{-} \alpha_{2_{12}}^{-} \alpha_{2_{21}}^{-}$is comparable to the rest of the squared amplitude. With reference to Eqs. (5.33) and (5.34) we thus introduce several sensitivity parameters $\mathcal{T}_{n}$

$$
\mathcal{T}_{n} \equiv \mathcal{X}_{n} /\left[\left|\alpha_{1_{11}}^{-} \alpha_{2_{11}}^{-}\right|^{2}+\sum_{\substack{j \neq k \\ r_{k} \neq s_{k}}}\left|\alpha_{j_{11}}^{-} \alpha_{k_{r s}}^{-}\right|^{2}+\mathcal{X}_{n}\right],
$$


where $n=M^{(\prime)}, R^{(\prime)}, J^{(\prime)}$, with

$$
\begin{gathered}
\mathcal{X}_{\mathrm{M}}=4\left|\alpha_{1_{12}}^{-} \alpha_{1_{21}}^{-} \alpha_{2_{12}}^{-} \alpha_{2_{21}}^{-}\right|, \quad \mathcal{X}_{\mathrm{R}}=4 \operatorname{Re}\left[\alpha_{1_{12}}^{-} \alpha_{1_{21}}^{-} \alpha_{2_{12}}^{-} \alpha_{2_{21}}^{-}\right], \quad \mathcal{X}_{\mathrm{J}}=4 \operatorname{Im}\left[\alpha_{1_{12}}^{-} \alpha_{1_{21}}^{-} \alpha_{2_{12}}^{-} \alpha_{2_{21}}^{-}\right] \\
\text {and } \quad \mathcal{X}_{\mathrm{M}^{\prime}, \mathrm{R}^{\prime}, \mathrm{J}^{\prime}}=\sum_{\substack{j \neq k \\
r \neq s, \rho \neq \sigma}}\left|\alpha_{j_{r s}}^{-} \alpha_{k_{\rho \sigma}}^{-}\right|^{2}+\mathcal{X}_{\mathrm{M}, \mathrm{R}, \mathrm{J}} .
\end{gathered}
$$

The first two terms of the denominator in Eq. (5.52) are simply the $\xi$ independent parts of the HBH squared amplitude (5.31), while the $\mathcal{X}_{n}$ 's are various pieces of the interference terms. In particular, $\mathcal{T}_{\mathrm{M}}$ is the magnitude of the full leading order interference term, while $\mathcal{T}_{\mathrm{R}, \mathrm{J}}$ are respectively the CP-even and CP-odd interference terms. We expect each to be useful gauge of sensitivity to CPV independent of $\xi$. For example, cuts on $\mathcal{T}_{\mathrm{R}}$ and $\mathcal{T}_{\mathrm{J}}$ will enhance the azimuthal modulations in the $\xi=0$ and $\xi=\pi / 4$ respectively, and can thus be used to distinguish among these. In all cases $\mathcal{T}_{i} \rightarrow 1\left(\mathcal{T}_{i} \rightarrow 0\right)$ implies full (no) CPV sensitivity.

For all numerical analysis we use three private Monte Carlo codes that were crosschecked. The details on Monte Carlo event generation can be found in Appendix C.VI. Placing a cut on $\mathcal{T}_{n}$ produces an event sample with large $\langle\mathcal{B}\rangle /\langle\mathcal{A}\rangle$ ratio. This is shown in Fig. 5.9 for opening angle cut $\theta_{\ell \ell}>10^{-5}$ and in Fig. 5.10 for opening angle cut of $\theta_{\ell \ell}>10^{-4}$. The $d \Gamma / d \varphi$ distributions are shown for two choices of CPV parameters, $\xi=0$ (blue histograms) and $\xi=\pi / 4$ (red histograms). The fits to the functional form (5.48) of the $d \Gamma / d \varphi \mathrm{HBH}$ differential rate are shown as solid blue and dot-dashed red lines, respectively. The efficiencies of the cuts for the examples shown in Figs. 5.9 and 5.10 are $\sim 1 \%$ for the upper panels and $\sim 0.1 \%$ for the lower panels (the exact values of efficiencies depend on the value of $\xi$ ). The presence of $\xi \neq 0$ exhibits itself as the expected phase shift in $d \Gamma / d \varphi$ differential rate (5.48).

From Figs. 5.9 and 5.10 we see that it is possible to select regions of phase space such that $\langle\mathcal{B}\rangle /\langle\mathcal{A}\rangle \sim \mathcal{O}(1)$. In particular the average modulation $\langle\mathcal{B}\rangle /\langle\mathcal{A}\rangle$ is large (small) for 

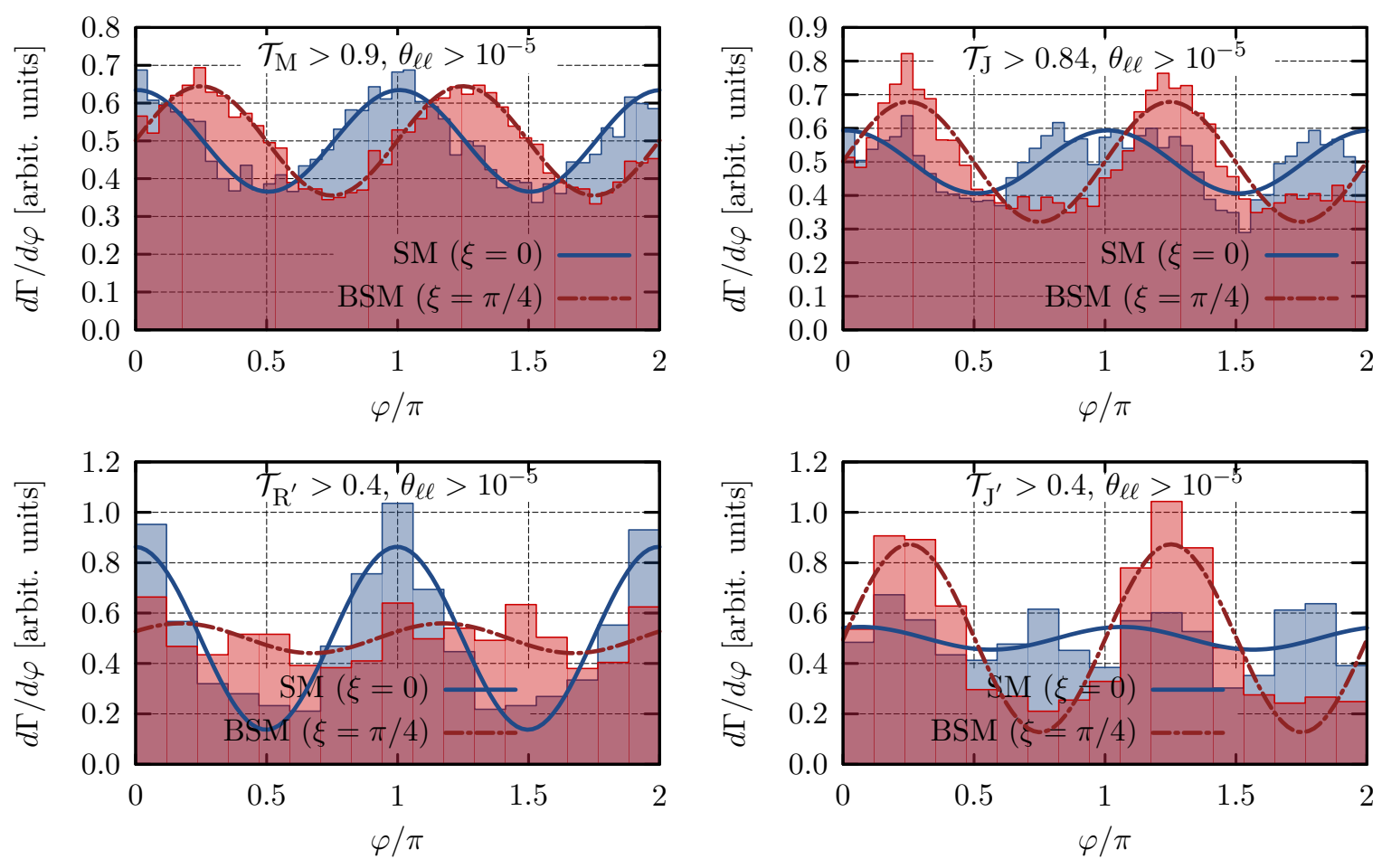

Figure 5.9: The differential $\mathrm{HBH}$ rate $d \Gamma / d \varphi$ as a function of the azimuthal angle $\varphi$ between the positrons setting the CPV parameter to $\xi=0$ (blue histograms) and $\xi=$ $\pi / 4$ (red histograms). The histograms are binned Monte Carlo events with different cuts on parameters $\mathcal{T}_{n}$, Eq. (5.52), as denoted in the panels. The solid blue (red dotdashed) curves are the result of fitting the normalized binned events to Eq. (5.50) for the $\xi=0(\xi=\pi / 4)$ cases with $\xi$ also floated in the fit. The angular resolution cut is $\theta_{\ell \ell}>\theta_{\text {cut }}=10^{-5}$.

$\xi=0(\xi=\pi / 4)$ for $\mathcal{T}_{\mathrm{R}, \mathrm{R}^{\prime}}$ and vice versa for $\mathcal{T}_{\mathrm{J}, \mathrm{J}^{\prime}}$ as shown most strikingly in the bottom panels. In contrast, the average modulation size does not depend on $\xi$ for the events selected by $\mathcal{T}_{\mathrm{M}}$. This suggests that using several of the parameters $\mathcal{T}_{n}$ simultaneously may optimize the sensitivity to CPV further.

\subsubsection{Cuts on individual photon conversions}

We now turn to discuss cuts on individual photon branches of the $\mathrm{HBH}$ process. These cuts are generated by simple sufficiency conditions, that ensure a large CPV signal.

From Eqs. (5.31) and (5.32), in order to ensure that the $\xi$-dependent terms are com- 

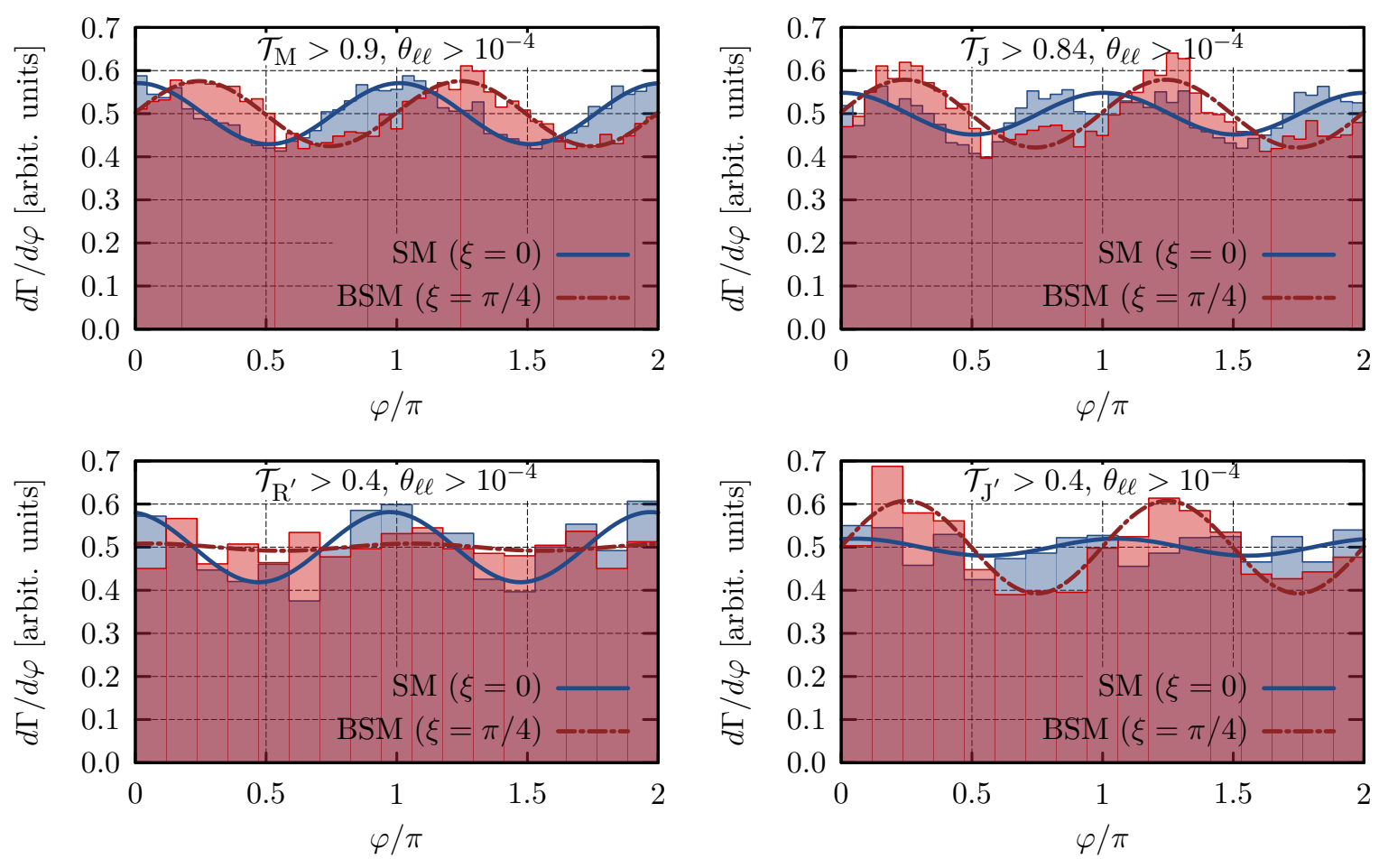

Figure 5.10: The same as in Fig. 5.9, but with the opening angle cut $\theta_{\ell \ell}>\theta_{\text {cut }}=10^{-4}$.

parable to the full rate, it suffices to require $\left|\alpha_{i_{12}}^{-} \alpha_{i_{21}}^{-}\right| \gtrsim\left|\overline{i_{i_{1}}}\right|^{2}$ on each branch $(i=1,2)$ independently. For the case that the Higgs is at rest, using (5.36), this sufficiency condition is implied by $\mathcal{S} \gtrsim 1$ for each branch, where

$$
\mathcal{S} \equiv 2(1-\cos \delta)\left[\frac{\gamma_{+} \gamma_{-}}{\left(\gamma_{+}+\gamma_{-}\right)^{2}}\right]\left[\frac{\gamma_{+} \theta_{+} \gamma_{-} \theta_{-}}{\left(\gamma_{+}^{2} \theta_{+}^{2}-\gamma_{-}^{2} \theta_{-}^{2}\right)^{2}}\right]\left(1+\gamma_{+}^{2} \theta_{+}^{2}\right)\left(1+\gamma_{-}^{2} \theta_{-}^{2}\right)
$$

For brevity, we have dropped the Latin branch index, and here $\delta \equiv\left|\phi_{-}-\phi_{+}\right|-\pi$ is the acoplanarity angle.

It is notable that this sufficiency condition weights the desirable energy-polar angle regions of phase space inversely by the amount of acoplanarity. Specifically, $\mathcal{S}=0$ in the exact coplanar configuration $\delta=0$. Of course, $\mathcal{S}$ is not a necessary condition for a large CPV signal, so relatively coplanar events - i.e. events for which $\delta \ll \delta_{\text {cut }}$ of the event sample ( $\delta_{\text {cut }}=0.4 \pi$ or $0.25 \pi$ for our MC, see App. C.VI) - may in principle 
significantly contribute to large CPV signals in $d \Gamma / d \varphi$. Also note that symmetric conversions, i.e. $\gamma_{+} \theta_{+} \simeq \gamma_{-} \theta_{-}$, are more likely to produce a large $\mathcal{S}$, and hence a strong interference effect.

Fig. 5.11 shows the results of the $\mathcal{S}_{1,2}>1$ cut for the angular resolution cut (5.38) $\theta_{\text {cut }}=10^{-5}$ for $\xi=0$ compared to $\xi=\pi / 20$ (top left) or $\pi / 4$ (top right). Note we also constrain the acoplanarity $\delta_{1,2} \in[3 \pi / 4,5 \pi / 4]$, since we do not expect extremely acoplanar events to encode polarization information. The angular resolution cut alone retains approximately $40 \%$ of the total $\mathrm{HBH}$ events on this acoplanar domain; for $\xi=$ $\pi / 4$, the corresponding azimuthal distribution is shown in Fig. 5.8. The addition of the $\mathcal{S}_{1,2}>1$ cut reduces the cut efficiency to $2.6 \%$, but unlike Fig. 5.8, now $\langle\mathcal{B}\rangle /\langle\mathcal{A}\rangle \sim$ $\mathcal{O}(0.2)$. The phase shift due to non-zero $\xi$ is clearly visible in both plots, and the value of $\xi$ extracted from the fits agrees with the input values of $\xi=\pi / 20$ or $\pi / 4$ respectively.

Finally, the bottom panels of Fig. 5.11 displays the acoplanarity on each branch for the $\xi=\pi / 4 \mathrm{HBH}$ events with no $\mathcal{S}$ cut compared to the $\mathcal{S}_{1,2}>1$ cuts. We see that the $\mathcal{S}$ cut mildly favors acoplanar events, as it broadens the acoplanarity distribution and disfavors relatively coplanar events. For example, we see that events with acoplanarity $\delta<1 \%$ are disfavored in the cut distribution, and we also see that the full width at half maximum of the acoplanar distribution increases by $50 \%$, from $0.04 \pi$ to $0.06 \pi$, under the $\mathcal{S}>1$ cut. The excellent performance of $\mathcal{S}$ compared to $\mathcal{T}_{n}$ cuts (see Fig. 5.12 and Sec. 5.5.4.3 below) therefore suggests that acoplanar events - e.g. with $\delta \nless \delta_{\text {cut }}$ - play an important role in encoding the CPV signal.

\subsubsection{Cut scheme efficiencies}

It remains to determine the efficiency of the above cut schemes. In Fig. 5.12 we show the CPV signal $\langle\mathcal{B}\rangle /\langle\mathcal{A}\rangle$ for the various $\mathcal{T}_{n}$ and $\mathcal{S}$ schemes as a function of the fraction of the total MC event sample, not rejected by combined sensitivity and angular resolution cuts. Up to small corrections this fraction is the absolute cut efficiency, see Appendix 

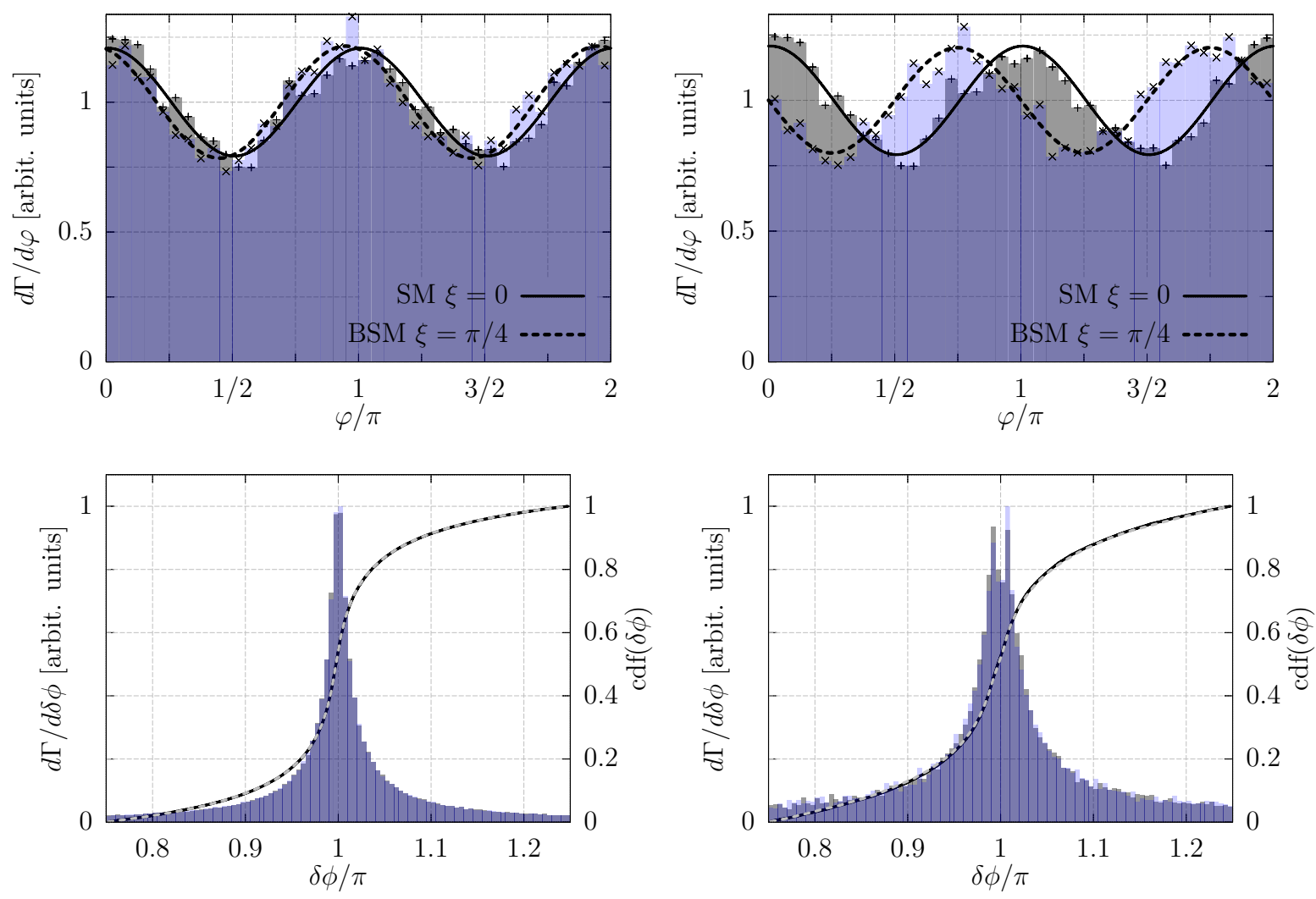

Figure 5.11: Top panels: The azimuthal distributions $d \Gamma / d \varphi$ for $\xi=0$ (grey histograms) and for $\xi=\pi / 20, \pi / 4$ (blue histograms, left and right top panels) with $\mathcal{S}_{1,2}>1$ on the domain $\delta_{1,2} \in[3 \pi / 4,5 \pi / 4]$. The solid (dashed) curves denote fits to Eq. (5.48), with $\xi$ a free parameter in the fits. The bottom left (right) panel shows the acoplanarity distributions, $d \Gamma / d \delta \phi, \delta \phi \equiv\left(\phi_{+}-\phi_{-}\right)$mod $2 \pi$ for each photon branch, displayed by blue and gray histograms respectively, with $\xi=\pi / 4$ and no $\mathcal{S}$ cut $\left(\mathcal{S}_{1,2}>1\right)$. Note that the scale varies between these two plots. The corresponding cumulative acoplanarity distributions $\operatorname{cdf}(\delta \phi)$ for each branch are denoted by solid black and grey dashed lines on each plot. In all panels the angular resolution cut (5.38) of $\theta_{\text {cut }}=10^{-5}$ was applied.

C.VI for details. For comparison, the data point with only angular resolution cuts is also shown for each plot.

We see that an increase of the CPV signal by an order of magnitude roughly requires an order of magnitude penalty in sample size. Moreover, for high cut efficiencies, the $\mathcal{S}_{i}$ scheme provides the largest CPV signal. In contrast, in the low efficiency, but higher signal regions, the $\mathcal{T}_{n}$ schemes outperform the $\mathcal{S}_{i}$ cuts. To give an idea about the feasibility of this analysis at the (HL/HE-)LHC, Table 5.1 gives the expected number of 


\begin{tabular}{cccc}
\hline \hline$\sqrt{s}$ & $\mathcal{L}\left[\mathrm{fb}^{-1}\right]$ & $\sigma \times \mathrm{BR}(h \rightarrow \gamma \gamma)[\mathrm{fb}]$ & Events \\
\hline 8 & 20 & 47 & 0.24 \\
14 & 3000 & 125 & 94 \\
33 & 3000 & 444 & 333 \\
100 & 3000 & 1875 & 1406 \\
\hline \hline
\end{tabular}

Table 5.1: Expected number of events after the application of $\mathcal{S}$ or $\mathcal{T}$ cuts with $\theta_{\ell \ell}>10^{-4}$ to obtain $\langle\mathcal{B}\rangle /\langle\mathcal{A}\rangle \sim 20 \%$. The Higgs production cross section includes the gluon fusion and VBF channels only and is taken from [287].

events after the application of the cuts discussed above. These numbers do not inlcude experimental acceptance efficiencies and so will be lower in practice.

The $\mathcal{S}_{i}$ parameter is defined on an individual photon branch, independently of the lepton configurations on the other branch, while $\mathcal{T}_{n}$ is defined on the configuration of whole $\mathrm{HBH}$ events. We therefore might deduce that the main difference between these two schemes is whether they are affected by inter-branch leptonic configurations. If this deduction is correct, then the comparative performance of $\mathcal{S}_{i}$ versus $\mathcal{T}_{n}$ cuts, shown in Fig. 5.12, suggest that inter-branch configurations become more important for the extraction of larger CPV signals, but are not important over most of the CPV sensitive phase space.

\subsubsection{Triple products}

Finally, we briefly comment on the possibility to use CP-odd quantities such as triple products to directly search for $\mathrm{CPV}$. Since the $\mathrm{CP}$ odd terms in $|\mathcal{M}|^{2}$ are $C$ even and $P$ odd, we could consider two such contractions

$$
\tau_{1}=\epsilon^{p_{1-} p_{1_{+}} p_{2 \_} p_{2_{+}}},
$$

and

$$
\tau_{2}=\frac{1}{4}\left(\epsilon^{P p_{1} p_{2_{-}} p_{2_{+}}}+\epsilon^{p_{1} P p_{2_{-}} p_{2_{+}}}+\epsilon^{p_{1} p_{1_{+}} P p_{2_{+}}}+\epsilon^{p_{1_{-}} p_{1_{+}} p_{2_{-}} P}\right),
$$



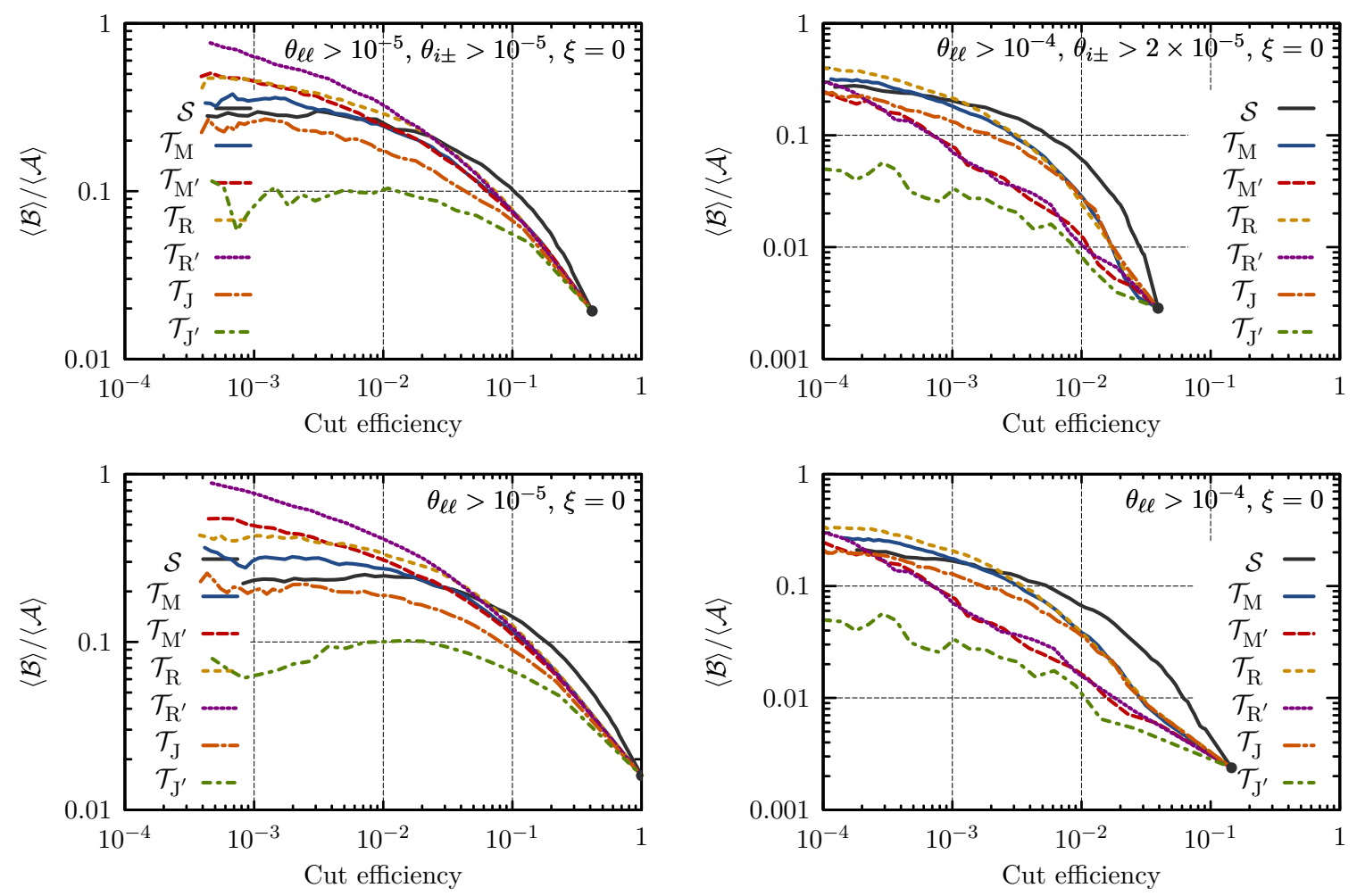

Figure 5.12: Comparison of different sensitivity parameter cut schemes, in terms of cut efficiency, for various angular resolution cuts. The black data point on each plot denotes the efficiency and $\langle\mathcal{B}\rangle /\langle\mathcal{A}\rangle$ for its angular resolution cut alone, with no enhancements from sensitivity parameter cuts.

where $P^{\mu}=(M,(m) 0)$ is the nucleus momentum, and $\epsilon^{p q r s}$ is shorthand for the LeviCivita contraction $\epsilon^{\mu \nu \rho \sigma} p_{\mu} q_{\nu} r_{\rho} s_{\sigma}$. In terms of scalar triple products,

$$
\begin{aligned}
& \tau_{1}=E_{1_{+}}(m) p_{1_{-}} \cdot\left((m) p_{2_{+}} \times(m) p_{2_{-}}\right)-E_{1_{-}}(m) p_{1_{+}} \cdot\left((m) p_{2_{+}} \times(m) p_{2_{-}}\right)+1 \leftrightarrow 2, \\
& \tau_{2}=M\left[(m) p_{1_{-}} \cdot\left((m) p_{2_{+}} \times(m) p_{2_{-}}\right)-(m) p_{1_{+}} \cdot\left((m) p_{2_{+}} \times(m) p_{2_{-}}\right)+1 \leftrightarrow 2\right] .
\end{aligned}
$$

Note, that $\tau_{2}$ is not strictly speaking C-even, since it involves the nucleus momentum. However, $\mathrm{BH}$ conversion on nucleus is the same as on anti-nucleus within our uncertainties. This is equivalent to leaving $P^{\mu}$ unchanged under $\mathrm{C}$ transformation. A detailed analysis is beyond the scope of our work, but we remark in passing that a straightforward use of the $\mathcal{S}$ and $\mathcal{T}_{n}$ cuts does not lead to appreciable non-zero value 
of $\langle\tau\rangle$. Further investigation is warranted.

\subsection{Chapter summary}

In this work we have studied how to probe the underlying CP couplings of the Higgs to two photons, which undergo Bethe-Heitler conversion on nuclei. We have shown that sensitivity to $\mathrm{CP}$ violating couplings is possible only if there is interference between conversion amplitudes with different photon helicity. Using spinor helicity methods, we have computed compact, leading order expressions for these amplitudes, which are novel to this work. Our analytical control of the leading order $h \rightarrow \gamma \gamma \stackrel{B H}{\rightarrow} 4 e$ full differential scattering rate permits us to show that: (i) the differential rate with respect to the relative azimuthal angles between leptons with different parent photons is oscillatory; (ii) CPV is encoded as a phase shift in such distributions; and (iii) we may construct various sensitivity parameter cuts that extract the regions of phase space on which such oscillations - the CPV signal - are order unity on average. These analytical results have been confirmed and explored with numerical simulations, including a comparison of the relative effectiveness of the different sensitivity parameters.

For simplicity we restricted our numerical and CP sensitivity analysis to the case that the Higgs is at rest in the lab frame. This is not the case in the LHC experiments, and this assumption needs to be lifted for more realistic studies. We note, in this vein, that Eqs. (C.13) hold also for the boosted Higgs case, although Eqs. (5.36) do not. The sensitivity parameter cut schemes are expected to work comparatively well in the case that the Higgs is boosted, too. On a similar note, we expect these sensitivity cuts to enhance the full matrix element method. This method, as characterized by the param-

eter $Z_{B}^{c}$, appears to be a few times more sensitive to CPV than experiments measuring a single relative azimuthal angle. This leaves open the possibility of further improved CPV signals, compared to those shown in this work. 
We do not expect a $h \rightarrow \gamma \gamma \stackrel{B H}{\rightarrow} 4 e$ experiment to be straightforward, and further experimental and theoretical studies are needed in order to see if the methods discussed in this chapter can be used in practice. From an experimental viewpoint, it needs to be determined how well one can reconstruct the electron and positron momenta for opening angles $\sim 10^{-4}$ or even down to $\sim m / m_{h} \sim 10^{-5}$, and whether there are significant rescatterings after $\mathrm{BH}$ production. Already from our preliminary studies it is clear that large statistical datasets and fine granularity of the detectors will be needed, such as at the proposed HE-LHC, VLHC or TLEP [288]. Experimentally, the situation may be more favorable in such a machine, having a larger amount of statistics and better kinematic control of the Higgs. A completely independent direction for measuring the $\mathrm{CP}$ violating coupling of Higgs to photons - and a direction not explored in this chapter - would be the use of polarized photon beams at a photon collider. From a theoretical viewpoint, it also remains to determine and search for other CPV sensitive observables apart from the azimuthal distributions discussed here.

Lastly, while we focussed on Higgs diphoton decays in this work, most of this analysis holds for any pair of correlated photons. They can arise from a resonance, like the Higgs, or from scattering events. While the details have to be determined in a case-by-case basis, the principles, such as helicity interference, are the same. 


\section{Chapter 6}

\section{Probing the photon polarization in $B \rightarrow K^{*} \gamma$ with conversion}

\subsection{Introduction}

Measurement of the photon polarization in $b \rightarrow s \gamma$ radiative decays has been of longstanding interest. Within the Standard Model (SM), flavor-changing electroweak interactions maximally violate parity, so that one expects the fraction of left-handed photons in $b \rightarrow s \gamma$ processes to be order unity, up to small corrections arising from either the non-zero strange quark mass or from higher order QCD contributions. In contrast, certain New Physics (NP) scenarios may generate $b \rightarrow s \gamma$ operators of comparable size to the SM terms, but with exotic parity structure, significantly modifying the expected ratio of left- versus right-handed photons - the photon polarization ratio. Measurement of this ratio therefore has the potential to test the parity structure of $b \rightarrow s \gamma$ operators against SM expectations, as well as either constrain or detect the signatures of such NP scenarios.

The $b \rightarrow s \gamma$ photon polarization ratio may be measured via various different approaches. Dominantly right-handed photon production in resonant $B \rightarrow\left(K_{1}(1400) \rightarrow\right.$ 
$K \pi \pi) \gamma$ generates an up-down asymmetry of the photon momentum with respect to the $K_{1} \rightarrow K \pi \pi$ decay plane $[289,290]$. This up-down asymmetry was recently measured by the LHCb collaboration [291]. However, a theoretical prediction for the asymmetry is not yet available, so that the photon polarization ratio cannot yet be extracted from these results or compared to SM expectations. Along similar lines, the photon polarization ratio may also be probed by measuring the spin fraction of $\Lambda^{\prime}$ s in unpolarized $\Lambda_{b} \rightarrow \Lambda \gamma$ decays [292], or by measuring an angular asymmetry between the $\Lambda_{b}$ spin and the outgoing photon momentum for polarized $\Lambda_{b} \rightarrow \Lambda \gamma$ [293]. Other methods look for time dependent $\mathrm{CP}$ violation induced by mixing of $B \rightarrow K^{*} \gamma$ and $\bar{B} \rightarrow K^{*} \gamma$, which is proportional to the photon polarization ratio [294,295]. Additionally one may probe the polarization ratio by looking for asymmetries in angular observables in resonant $B \rightarrow K \pi \ell^{+} \ell^{-}$or $B \rightarrow \pi \pi \ell^{+} \ell^{-}[295,296]$, or look for transverse asymmetries in the dilepton invariant mass for non-resonant $B \rightarrow K \pi \ell^{+} \ell^{-}$[297].

In this work we focus on the $B \rightarrow\left(K^{*} \rightarrow K^{+} \pi^{-}\right) \gamma$ process, in which the on-shell photon subsequently undergoes Bethe-Heitler (BH) nuclear conversion inside the detector to a lepton-antilepton pair. The cross-section for $\mathrm{BH}$ pair conversion of $\sim \mathrm{GeV}$ photons is approximately two (eight) orders of magnitude larger than the Compton (Rayleigh) scattering cross-section ( [55]; see chapter 32), so that to an excellent approximation the emitted photon does not decohere before conversion. The $B \rightarrow K^{*} \gamma$ photon polarization ratio, $r$, is precisely defined via the amplitude ratio

$$
r e^{i(\phi+\delta)} \equiv \frac{\mathcal{A}\left(B \rightarrow K^{*} \gamma_{L}\right)}{\mathcal{A}\left(B \rightarrow K^{*} \gamma_{R}\right)}
$$

in which $\phi(\delta)$ is a relative weak (strong) phase. In the $\mathrm{SM}, r$ is expected to be at most $\sim \Lambda_{\text {qcd }} / m_{b}$ [298], while the weak phase $\phi$ is suppressed by $\Lambda_{\text {qcd }} / m_{b}\left|V_{u b} V_{u s}^{*} / V_{t b} V_{t s}^{*}\right| \ll$ 1. Hence measurement of not only $r$, as discussed above, but also $\phi$ may test SM expectations: Measuring either $r$ or $\phi \sim \mathcal{O}(1)$ would be highly suggestive of NP effects. 
Measurement of $r$ via BH conversion was first considered in Ref. [255]. In that analysis, the $B$ meson was assumed to be at rest relative to the conversion nucleus. Further, the conversion itself was assumed be to a perfect linear polarizer of the photon, so that the conversion leptons and photon are constrained to be coplanar. However, in practice the $B$ meson is typically at least semi-relativistic, and typically an $\mathcal{O}(1)$ fraction of conversion events have non-negligible acoplanarity (see e.g. Ref. [299]). This leads to a richer phase space structure for the outgoing conversion leptons, kaon and pion. Moreover, interference effects between the $B \rightarrow K^{*} \gamma$ decay and the $\mathrm{BH}$ conversion amplitudes were not included. The key motivation to reconsider the above analysis, then, is to include these $B$-boost, acoplanarity and interference effects. We exploit recent compact results for BH conversion spin-helicity amplitudes [299] to construct the full $B \rightarrow\left(K^{*} \rightarrow K^{+} \pi^{-}\right)\left(\gamma \stackrel{\mathrm{BH}}{\rightarrow} e^{+} e^{-}\right)$amplitude, and show that interference between its decay and conversion components permits both $r$ and $\phi$ to be probed by the kinematic configuration of the final state conversion leptons, kaon and pion. In particular, we develop an angular observable that probes both of these parameters. This new analysis admits arbitrary boosts of the parent $B$ meson relative to the $\mathrm{BH}$ conversion nucleus, and includes lepton-photon acoplanarity, which turns out to play an important role in enhancing the $r$ - and $\phi$-sensitive interference effects.

Performing an experiment to measure $r$ and $\phi$ with this technique will be challenging. In the first instance, precise reconstruction of the leptonic momenta is required, which can only be achieved, even in principle, if the leptonic opening angles are larger than the angular resolution of the detector. Typically, the leptonic opening angle after BH conversion is $\theta_{\ell \ell} \sim m_{e} / E_{\gamma} \sim 10^{-4}$, for typical photon energies in a $B \rightarrow K^{*} \gamma$ decay with a semirelativistic $B$. Specifically, for a photon of energy $\lesssim 5 \mathrm{GeV}$, the probability for $\theta_{\ell \ell}>10^{-4}\left(10^{-3}\right)$ is $\sim 98 \%(43 \%)$. Hence exquisite angular resolutions will be required. A further complicating factor is the multiple rescattering of the leptons in the detector material after conversion. The rms rescattering angle in matter is 
$\simeq\left(13.6 \mathrm{MeV} / E_{\ell}\right) \sqrt{x / x_{0}}$ [55], where $x / x_{0}$ is the path length inside the detector in units of radiation length. For $x / x_{0} \sim$ few $\%$ - a typical value - the rescattering angle is comparable to the typical opening angle, $\theta_{\ell \ell}$. Finally, the probability of photon conversion itself is typically low at current and planned $B$ factories, being at most of order a few percent. This probability depends mildly on the detector design. For example, it is approximately 3\%, 2-3\%, and 6\% at BaBar [300], LHCb [301], and Belle II [302] respectively. For all these reasons, this technique will likely only be feasible with a dedicated detector element that has a large scattering length, e.g. a gaseous TPC [303].

For these reasons, in this work we shall restrict ourselves to a thought experimenttype approach. That is, we develop explicit analytic expressions for amplitudes and observables with respect to the underlying $B \rightarrow\left(K^{*} \rightarrow K^{+} \pi^{-}\right)\left(\gamma \stackrel{\mathrm{BH}}{\rightarrow} e^{+} e^{-}\right)$process alone, but do not include smearing from leptonic rescattering and limited kinematic resolution, or realistic detector simulations. Based on the results of this work, future studies may perhaps incorporate these latter effects.

\subsection{Amplitudes}

\subsubsection{Amplitude factorization}

Keeping operators up to dimension five, the effective theory of interest for $B \rightarrow K^{*} \gamma$ and $\bar{B} \rightarrow \bar{K}^{*} \gamma$ decays may be written in the general form

$$
\mathcal{L}_{\text {eff }}=g_{\|} B^{\dagger} K_{\mu \nu}^{*} F^{\mu \nu}+g_{\perp} B^{\dagger} K_{\mu \nu}^{*} \tilde{F}^{\mu \nu}+\bar{g}_{\|} \bar{B}^{\dagger} \bar{K}_{\mu \nu}^{*} F^{\mu \nu}+\bar{g}_{\perp} \bar{B}^{\dagger} \bar{K}_{\mu \nu}^{*} \tilde{F}^{\mu \nu}
$$

where $\tilde{X}^{\mu \nu} \equiv \epsilon^{\mu \nu \rho \sigma} X_{\rho \sigma} / 2$, and the dimensionful couplings generically contain relative strong phases. We consider only $K^{*}$ decays to charged pseudoscalars, i.e. $K^{*} \rightarrow K^{+} \pi^{-}$ or $\bar{K}^{*} \rightarrow K^{-} \pi^{+}$. The sign of the pion or kaon charge therefore tags the $K^{*}$ versus 
the $\bar{K}^{*}$, and hence tags the parent meson as either a $B$ or $\bar{B}$, up to electroweak loop suppressed corrections. Hence we neglect interference effects from $B-\bar{B}$ mixing.

We assume the conversion nucleus is spin-0, e.g. a ${ }^{28} \mathrm{Si}$ nucleus, which is the dominant silicon isotope. The external quantum numbers for the full $B \rightarrow\left(K^{*} \rightarrow K^{+} \pi^{-}\right)(\gamma \stackrel{\mathrm{BH}}{\rightarrow}$ $\left.e^{+} e^{-}\right)$helicity amplitudes are then just the spins of the electron and positron, denoted $r$ and $s=1,2$ respectively. It follows that the full helicity amplitudes

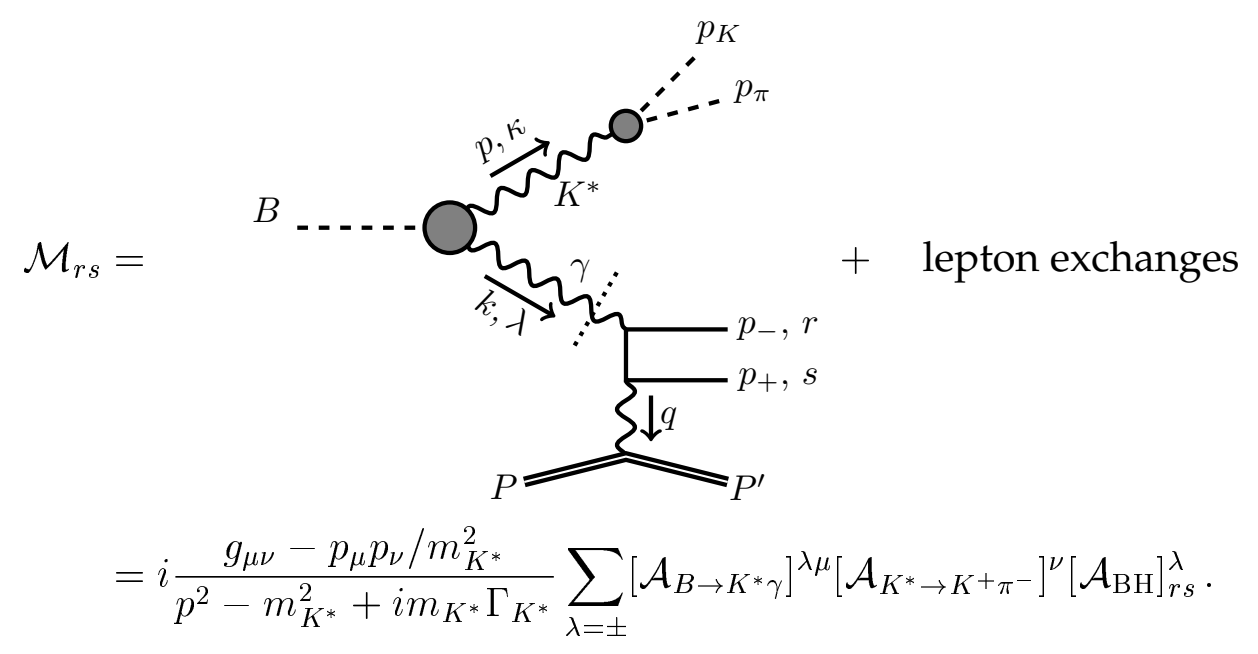

nearby to the $K^{*}$ Breit-Wigner peak. Here $\lambda= \pm(\kappa= \pm, 0)$ is the helicity of the photon $\left(K^{*}\right)$, and $k^{\mu}\left(p^{\mu}\right)$ is the photon $\left(K^{*}\right)$ momentum; $P\left(P^{\prime}\right)$ denotes the incoming (outgoing) nuclear momentum, with nuclear mass $P^{2}=P^{\prime 2}=M^{2} ; p_{ \pm}\left(p_{K, \pi}\right)$ denote the momenta of the leptons (kaon and pion); and finally $q$ denotes the momentum exchange with the nucleus.

Momentum and angular momentum conservation in the $B \rightarrow K^{*} \gamma$ process ensure that $A_{B \rightarrow K^{*} \gamma}$ must annihilate the longitudinal component of the $K^{*}$ propagator. Applying the polarization completeness relation for $p^{2} \neq 0$,

$$
\sum_{\kappa= \pm, 0} \epsilon_{\mu}^{\kappa}(p) \epsilon_{\nu}^{\kappa *}(p)=-g_{\mu \nu}+p_{\mu} p_{\nu} / p^{2}
$$


we may factorize the full helicity amplitude into three helicity amplitude factors,

$$
\mathcal{M}_{r s}=\frac{i}{p^{2}-m_{K^{*}}^{2}+i m_{K^{*}} \Gamma_{K^{*}}} \sum_{\lambda, \kappa}\left[\mathcal{A}_{B \rightarrow K^{*} \gamma}\right]^{\lambda \kappa}\left[\mathcal{A}_{K^{*} \rightarrow K^{+} \pi^{-}}\right]^{\kappa}\left[\mathcal{A}_{\mathrm{BH}}\right]_{r s}^{\lambda} .
$$

Similarly for the $\bar{B} \rightarrow\left(\bar{K}^{*} \rightarrow K^{-} \pi^{+}\right)\left(\gamma \stackrel{\mathrm{BH}}{\rightarrow} e^{+} e^{-}\right)$process

$$
\overline{\mathcal{M}}_{r s}=\frac{i}{p^{2}-m_{K^{*}}^{2}+i m_{K^{*}} \Gamma_{K^{*}}} \sum_{\lambda, \kappa}\left[\mathcal{A}_{\bar{B} \rightarrow \bar{K}^{*} \gamma}\right]^{\lambda \kappa}\left[\mathcal{A}_{\bar{K}^{*} \rightarrow K^{-} \pi^{+}}\right]^{\kappa}\left[\mathcal{A}_{\mathrm{BH}}\right]_{r s}^{\lambda} .
$$

Here and hereafter we neglect the mass splittings of the $B-\bar{B}$ and $K^{*}-\bar{K}^{*}$ systems, and denote the masses (momenta) of both $\mathrm{CP}$ conjugate states by $m_{B}$ and $m_{K^{*}}\left(p_{B}\right.$ and $p$ ) respectively.

\subsubsection{Kinematics}

The amplitude factors in eqs. (6.5) are Lorentz invariants, and are naturally expressed with respect to kinematic coordinates that are defined in different frames. That is, the full $2 \rightarrow 5$ phase space - the full coherent process is $B N \rightarrow K^{+} \pi^{-} \ell^{+} \ell^{-} N$, for nucleus $N$ - is factored into two $1 \rightarrow 2$ phase spaces, corresponding to $B \rightarrow K^{*} \gamma$ and $K^{*} \rightarrow K^{+} \pi^{-}$, and one $2 \rightarrow 3$ phase space for the $\mathrm{BH}$ conversion. In general, a $2 \rightarrow 5$ phase space is fully specified by eleven coordinates. Two of these are the photon and $K^{*}$ invariant masses. The former is fixed to $k^{2}=0$ for an on-shell internal photon. With regard to the $K^{*}$ invariant mass, it is convenient to define hereafter the dimensionless quantity

$$
\hat{s} \equiv p^{2} / m_{K^{*}}^{2}
$$

In the narrow width limit, the Breit-Wigner factor

$$
\frac{1}{\left|(\hat{s}-1) m_{K^{*}}^{2}+i m_{K^{*}} \Gamma_{K^{*}}\right|^{2}} \rightarrow \pi \frac{1}{m_{K^{*}} \Gamma_{K^{*}}} \delta\left[(\hat{s}-1) m_{K^{*}}^{2}\right] .
$$


That is, the narrow width limit corresponds to an on-shell $K^{*}$. Since, however, the $K^{*}$ has a finite width $-\Gamma_{K^{*}} / m_{K^{*}} \sim 5 \%$ - and need not be precisely on-shell, we shall treat $\hat{s}$ as a phase space variable: The Breit-Wigner ensures $\hat{s}$ is typically nearby to the $K^{*}$ mass shell up to the $K^{*}$ width, i.e. $\hat{s} \simeq 1$ up to variations $\sim \Gamma_{K^{*}} / m_{K^{*}}$.

In the case that the photon conversion material is cold, the lab frame coincides with the frame in which the $\mathrm{BH}$ conversion nucleus is at rest. The following choices, shown in Fig. 6.1, for the remaining nine coordinates then prove convenient for the construction of compact and intuitive results: the lepton polar and azimuthal angles $\theta_{ \pm}$and $\phi_{ \pm}$and the energies $E_{ \pm}$, defined in the nuclear rest frame - the lab frame - with respect to the photon momentum and the $K^{*}-\gamma$ decay plane, defined by $(m) p$ and $(m) k$; the photon polar angle, $\theta_{\gamma}$, defined with respect to the nuclear momentum, $(m) P$, in the $B$ rest frame; the $K$ polar and azimuthal angles $\theta_{K}$ and $\phi_{K}$, defined in the $K^{*}$ rest frame with respect to the photon momentum, $(m) k$, and the plane defined by $(m) P$ and $(m) k$ in that frame. Note that the $K^{*}-\gamma$ decay plane is invariant under boosts between the lab, $K^{*}$ and $B$ rest frames, and therefore equivalent to the plane defined by $(m) P$ and $(m) k$ in either the $K^{*}$ or $B$ rest frames, as shown in Fig. 6.1.

\subsubsection{Helicity amplitude factors}

With these choices, we now proceed to explicitly compute the amplitude factors $\left[M_{B \rightarrow K^{*} \gamma}\right]^{\lambda \kappa}$, $\left[M_{K^{*} \rightarrow K^{+} \pi^{-}}\right]^{\kappa}$, and $\left[M_{\mathrm{BH}}\right]_{r s}^{\lambda}$. Applying a light-cone decomposition to $p$, we define its associated null momentum with respect to the photon, i.e.

$$
\tilde{p}^{\mu} \equiv p^{\mu}-\frac{p^{2} k^{\mu}}{2 p \cdot k}
$$

and make the polarization gauge choices

$$
\epsilon_{K^{*}}^{ \pm}(p)= \pm \frac{\left\langle k^{\mp}\left|\sigma^{\mu}\right| \tilde{p}^{\mp}\right\rangle}{\sqrt{2}\left\langle k^{\mp} \mid \tilde{p}^{ \pm}\right\rangle}, \quad \epsilon_{\gamma}^{ \pm \mu}(k)= \pm \frac{\left\langle\bar{k}^{\mp}\left|\sigma^{\mu}\right| k^{\mp}\right\rangle}{\sqrt{2}\left\langle\bar{k}^{\mp} \mid k^{ \pm}\right\rangle}
$$



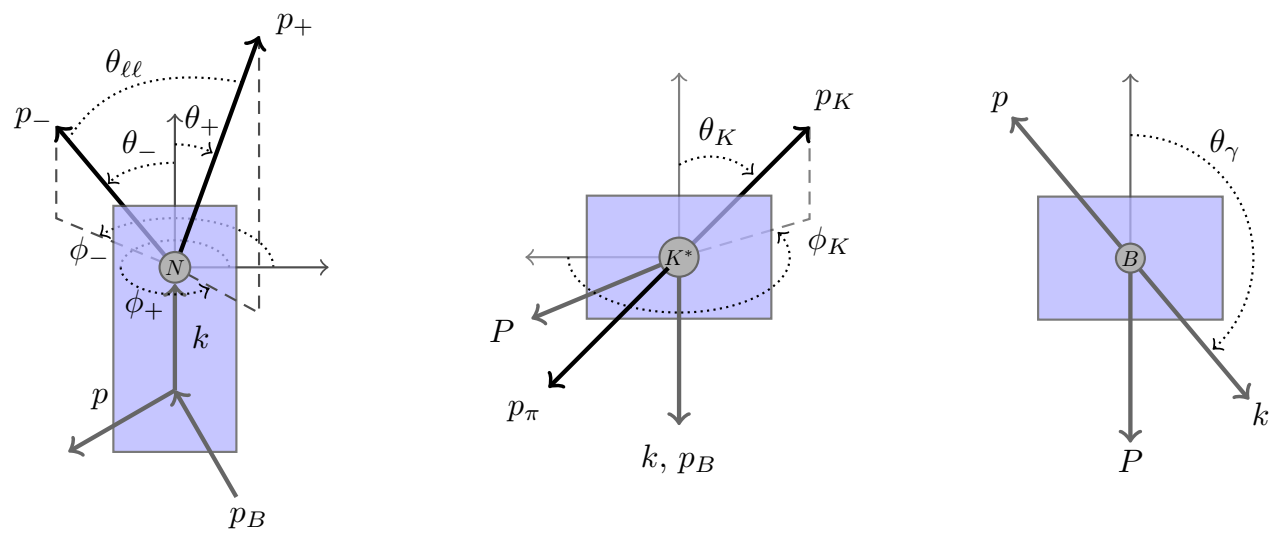

Figure 6.1: Kinematic configuration and coordinate choices. $B$ momentum is denoted by $p_{B}$, and azimuthal angles are defined with respect to the $K^{*}-\gamma$ decay plane (blue). This plane contains $(m) p$ and $(m) k((m) P$ and $(m) k)$ in the lab frame $\left(K^{*}\right.$ or $B$ rest frames); momenta lying in this plane in each frame are shown in gray. Left: Lepton polar angles $\theta_{ \pm}$and azimuthal angles $\phi_{ \pm}$in the lab frame. Middle: $\theta_{K}$ and $\phi_{K}$ polar angles in the $K^{*}$ rest frame. Right: The photon polar angle, $\theta_{\gamma}$, in the $B$ rest frame.

for $\bar{k}$ an arbitrary null reference momentum. From the effective theory (6.2) one may then show that the $B \rightarrow K^{*} \gamma$ and $\bar{B} \rightarrow \bar{K}^{*} \gamma$ helicity amplitudes are

$$
\begin{aligned}
& {\left[\mathcal{A}_{B \rightarrow K^{*} \gamma}\right]^{ \pm \pm}=\left(g_{\|} \pm i g_{\perp}\right)\left(m_{B}^{2}-m_{K^{*}}^{2} \hat{s}\right),} \\
& {\left[\mathcal{A}_{\bar{B} \rightarrow \bar{K}^{*} \gamma}\right]^{ \pm \pm}=\left(\bar{g}_{\|} \pm i \bar{g}_{\perp}\right)\left(m_{B}^{2}-m_{K^{*}}^{2} \hat{s}\right),}
\end{aligned}
$$

and $\left[M_{B \rightarrow K^{*} \gamma}\right]^{\mp \pm}=\left[M_{B \rightarrow K^{*} \gamma}\right]^{ \pm 0}=0$. Note that, by definition, $\mathcal{A}\left(B \rightarrow K^{*} \gamma_{R, L}\right) \equiv$ $\left[M_{B \rightarrow K^{*} \gamma}\right]^{ \pm \pm}$. It follows from eq. (6.1) and its CP conjugate that

$$
\frac{g_{\|}-i g_{\perp}}{g_{\|}+i g_{\perp}}=r e^{i(\delta+\phi)} \quad \text { and } \quad \quad \frac{\bar{g}_{\|}+i \bar{g}_{\perp}}{\bar{g}_{\|}-i \bar{g}_{\perp}}=r e^{i(\delta-\phi)}
$$


Hence

$$
\begin{aligned}
& {\left[\mathcal{A}_{B \rightarrow K^{*} \gamma}\right]^{++}=\left(g_{\|}+i g_{\perp}\right)\left(m_{B}^{2}-m_{K^{*}}^{2} \hat{s}\right),} \\
& {\left[\mathcal{A}_{B \rightarrow K^{*} \gamma}\right]^{--}=r e^{i(\delta+\phi)}\left(g_{\|}+i g_{\perp}\right)\left(m_{B}^{2}-m_{K^{*}}^{2} \hat{s}\right),} \\
& {\left[\mathcal{A}_{\bar{B} \rightarrow \bar{K}^{*} \gamma}\right]^{++}=r e^{i(\delta-\phi)}\left(\bar{g}_{\|}-i \bar{g}_{\perp}\right)\left(m_{B}^{2}-m_{K^{*}}^{2} \hat{s}\right),} \\
& {\left[\mathcal{A}_{\bar{B} \rightarrow \bar{K}^{*} \gamma}\right]^{--}=\left(\bar{g}_{\|}-i \bar{g}_{\perp}\right)\left(m_{B}^{2}-m_{K^{*}}^{2} \hat{s}\right) .}
\end{aligned}
$$

The reference gauge momentum $\bar{k}$ in eqs. (6.9) is so far arbitrary. However, a particularly convenient choice is

$$
\bar{k}^{\mu} \equiv \frac{2 P \cdot k}{M^{2}} P^{\mu}-k^{\mu}
$$

where $P^{2}=M^{2}$ is the nuclear mass. In the lab frame - the nuclear rest frame - this choice (6.13) ensures that for $k^{\mu}=E_{\gamma}(1,(\hat{m}) k)$ then simply $\bar{k}^{\mu}=E_{\gamma}(1,-(\hat{m}) k)$. We assume the nuclear scattering is coherent and quasi-elastic, i.e. that $P^{\prime 2}=M^{2}$ - equivalently $q^{0} \equiv P^{\prime 0}-P^{0}=q^{2} /(2 M)$ in the lab frame - and that the outgoing nucleus is non-relativistic, so that the momentum exchange with the nucleus $|(m) q| \ll M$ (see Refs $[272,273,304]$ for a review of $\mathrm{BH}$ conversion). With these assumptions and the choice of $\bar{k}$ in eq. (6.13), the BH spin-helicity amplitudes collapse to a simple form in the limit that the polar angles $\theta_{ \pm} \ll 1$ and Lorentz factors $\gamma_{ \pm} \equiv E_{ \pm} / m \gg 1$, where $m$ is the lepton mass [299]. At leading order in these limits,

$$
\left[\mathcal{A}_{\mathrm{BH}}\right]_{r s}^{\lambda} \simeq e^{3} \alpha_{r s}^{\lambda},
$$


with

$$
\begin{aligned}
& \alpha_{11}^{-}=-\left(\alpha_{22}^{+}\right)^{*}=2 \sqrt{2 \gamma_{+} \gamma_{-}} \frac{\sqrt{\mathcal{G}\left(q^{2}\right)}}{q^{2}}\left(\frac{1}{1+\gamma_{+}^{2} \theta_{+}^{2}}-\frac{1}{1+\gamma_{-}^{2} \theta_{-}^{2}}\right), \\
& \alpha_{\frac{12}{21}}^{-}=+\left(\alpha_{21}^{+}\right)^{*}= \pm 2 \sqrt{2 \gamma_{+} \gamma_{-}} \frac{\sqrt{\mathcal{G}\left(q^{2}\right)}}{q^{2}} \frac{\gamma_{\mp}}{\gamma_{+}+\gamma_{-}}\left(\frac{\gamma_{+} \theta_{+} e^{-i \phi_{+}}}{1+\gamma_{+}^{2} \theta_{+}^{2}}+\frac{\gamma_{-} \theta_{-} e^{-i \phi_{-}}}{1+\gamma_{-}^{2} \theta_{-}^{2}}\right), \\
& \alpha_{22}^{-}=-\left(\alpha_{11}^{+}\right)^{*}=0
\end{aligned}
$$

in which

$$
-q^{2} \simeq m^{2}\left(\gamma_{+}^{2} \theta_{+}^{2}+\gamma_{-}^{2} \theta_{-}^{2}+2 \gamma_{-} \gamma_{+} \theta_{-} \theta_{+} \cos \left(\phi_{-}-\phi_{+}\right)\right)+\frac{m^{2}}{4}\left[\frac{1}{\gamma_{+}}+\frac{1}{\gamma_{-}}\right]^{2} .
$$

Here $\mathcal{G}\left(q^{2}\right)$ is the $\mathrm{BH}$ quasi-elastic form factor for the photo-nuclear vertex [272,273],

$$
\mathcal{G}\left(q^{2}\right)=M^{2} a^{4} q^{4} /\left(1-a^{2} q^{2}\right)^{2}
$$

in which $a=184.15(2.718)^{-1 / 2} Z^{-1 / 3} / m$ and $Z$ is the atomic number of the nucleus. This form factor encodes electronic screening of the nucleus, and regulates the $1 / q^{2}$ pole in the amplitudes. From eqs. (6.15), one sees that the BH amplitudes are maximal at $\gamma_{ \pm} \theta_{ \pm} \sim 1$. That is, the typical lepton polar angle $\theta_{ \pm} \sim m / E_{ \pm}$. It also follows that the typical momentum exchange $-q^{2} \sim m^{2}$, i.e. $|(m) q|^{2} \ll M^{2}$ in concordance with our assumption of non-relativistic scattering.

It now remains to compute the $K^{*} \rightarrow K^{+} \pi^{-}$and $\bar{K}^{*} \rightarrow K^{-} \pi^{+}$helicity amplitudes. Only transverse $K^{*}$ modes are generated by the $B \rightarrow K^{*} \gamma$ amplitude. Since $\epsilon_{K^{*}}^{ \pm} \cdot p=0$ and $p=p_{K}+p_{\pi}$, these amplitudes must therefore take the form

$$
\left[\mathcal{A}_{K^{*} \rightarrow K^{+} \pi^{-}}\right]^{\kappa}=g_{K^{*}} \epsilon_{K^{*} \mu}^{\kappa}(p)\left(p_{K}^{\mu}-p_{\pi}^{\mu}\right)
$$

where $g_{K^{*}}$ is a dimensionless coupling, containing a strong phase. There are no other 
weak phases as $K^{*}$ decays strongly to $K \pi$. Under the polarization conventions (6.9), and computing in the $K^{*}$ rest frame defined by Fig. 6.1, the helicity amplitudes are just spherical harmonics

$$
\left[\mathcal{A}_{K^{*} \rightarrow K^{+} \pi^{-}}\right]^{ \pm}\left(\theta_{K}, \phi_{K}\right)=\frac{e^{ \pm i \phi_{K}}}{\sqrt{2}} g_{K \pi} p_{K \pi} \sin \theta_{K}
$$

with the momentum

$$
p_{K \pi} \equiv \frac{1}{\hat{s}^{1 / 2} m_{K^{*}}}\left[m_{K^{*}}^{2} \hat{s}-\left(m_{K}+m_{\pi}\right)^{2}\right]^{1 / 2}\left[m_{K^{*}}^{2} \hat{s}-\left(m_{K}-m_{\pi}\right)^{2}\right]^{1 / 2}
$$

Under $\mathrm{CP}$, note that the amplitude transforms as

$$
\left[\mathrm{CP} \mathcal{A}_{K^{*} \rightarrow K^{+} \pi^{-}}\right]^{ \pm}\left(\theta_{K}, \phi_{K}\right)=\left[\mathcal{A}_{\bar{K}^{*} \rightarrow K^{-} \pi^{+}}\right]^{\mp}\left(\theta_{K}, \phi_{K}\right)=\left[\mathcal{A}_{K^{*} \rightarrow K^{+} \pi^{-}}\right]^{ \pm}\left(\theta_{K},-\phi_{K}\right) .
$$

That is, defining $\phi_{K}$ and $\theta_{K}$ with respect to $\bar{K}^{*}$ rest frame just as for the $K^{*}$ in Fig. 6.1, then

$$
\left[\mathcal{A}_{\bar{K}^{*} \rightarrow K^{-} \pi^{+}}\right]^{ \pm}\left(\theta_{K}, \phi_{K}\right)=\frac{e^{ \pm i \phi_{K}}}{\sqrt{2}} \bar{g}_{K \pi} p_{K \pi} \sin \theta_{K}
$$

\subsubsection{Full Amplitude}

Applying all the results (6.12), (6.15), (6.19) and (6.22) to eqs. (6.5), and defining $|\alpha|^{2} \equiv$ $\sum_{\lambda, r, s}\left|\alpha_{r s}^{\lambda}\right|^{2}$, the unpolarized square amplitudes

$$
\begin{aligned}
& |\mathcal{M}|^{2}=A(r) \sin ^{2} \theta_{K}\left\{|\alpha|^{2}+\frac{8 r}{1+r^{2}} \operatorname{Re}\left[\alpha_{12}^{-} \alpha_{21}^{-} e^{i\left(\phi+\delta-2 \phi_{K}\right)}\right]\right\}, \\
& |\overline{\mathcal{M}}|^{2}=\bar{A}(r) \sin ^{2} \theta_{K}\left\{|\alpha|^{2}+\frac{8 r}{1+r^{2}} \operatorname{Re}\left[\alpha_{12}^{-} \alpha_{21}^{-} e^{i\left(\phi-\delta-2 \phi_{K}\right)}\right]\right\},
\end{aligned}
$$


in which

$$
\begin{aligned}
& A(r) \equiv \frac{e^{3}}{4}\left(1+r^{2}\right) \frac{\left|g_{\|}+i g_{\perp}\right|^{2}\left|g_{K \pi}\right|^{2}}{\left|m_{K^{*}}^{2}(\hat{s}-1)+i m_{K^{*}} \Gamma_{K^{*}}\right|^{2}} p_{K \pi}^{2}\left(m_{B}^{2}-m_{K^{*}}^{2} \hat{s}\right)^{2}, \\
& \bar{A}(r) \equiv \frac{e^{3}}{4}\left(1+r^{2}\right) \frac{\left|\bar{g}_{\|}-i \bar{g}_{\perp}\right|^{2}\left|\bar{g}_{K \pi}\right|^{2}}{\left|m_{K^{*}}^{2}(\hat{s}-1)+i m_{K^{*}} \Gamma_{K^{*}}\right|^{2}} p_{K \pi}^{2}\left(m_{B}^{2}-m_{K^{*}}^{2} \hat{s}\right)^{2} .
\end{aligned}
$$

Eqs. (6.23) compactly express the unpolarized square amplitude for the full $B \rightarrow\left(K^{*} \rightarrow\right.$ $\left.K^{+} \pi^{-}\right)\left(\gamma \stackrel{\mathrm{BH}}{\rightarrow} e^{+} e^{-}\right)$process in terms of just the $\mathrm{BH}$ conversion helicity amplitudes (6.15) and trigonometric (exponential) functions of the kinematic observables $\theta_{K}\left(\phi_{K}\right)$. The dependence on the parameters $r, \phi$ and $\delta$ is explicit and elementary.

As a cross-check of these results, we provide an alternative and more traditional derivation of the square amplitude in Appendix D.I, via construction of linearly polarized photon BH amplitudes. The consequent result (D.4) and the square amplitude (6.23) are in excellent numerical agreement in the $\gamma_{ \pm} \gg 1$ and $\theta_{ \pm} \ll 1$ regime, applicable to the $B \rightarrow\left(K^{*} \rightarrow K^{+} \pi^{-}\right)\left(\gamma \stackrel{\mathrm{BH}}{\rightarrow} e^{+} e^{-}\right)$process. Note that the result (D.4) does not incorporate these approximations, so that the compact and explicit eqs. (6.23) are strictly an approximation to eq. (D.4).

\subsection{Observables}

\subsubsection{Differential rate}

Making use of the explicit $r$ and $\phi$ dependence in the square amplitude results (6.23), we may now proceed to extract $r$ and $\phi$ sensitive observables. First, however, we construct the full differential rate. The factorization (6.5) ensures that the phase space with an on-shell internal photon may be partitioned into a $B \rightarrow\left(K^{*} \rightarrow K \pi\right) \gamma$ cascade decay and a $\gamma N \rightarrow \ell^{+} \ell^{-} N$ conversion. That is, the differential rate for the full 
$B \rightarrow\left(K^{*} \rightarrow K^{+} \pi^{-}\right)\left(\gamma \stackrel{\text { BH }}{\rightarrow} e^{+} e^{-}\right)$process can be written as

$$
d \mathcal{R}=|\mathcal{M}|^{2} d \mathcal{P}_{B} d \mathcal{P}_{\mathrm{BH}}
$$

where $d \mathcal{P}_{B}\left(d \mathcal{P}_{\mathrm{BH}}\right)$ is phase space factor of the decay (conversion). Note that $d \mathcal{R}$ here has the dimensions of a cross-section times a partial width.

Each phase space factor is Lorentz invariant, and are naturally computed in different frames, as shown in Fig. 6.1. Computing in the $B$ rest frame followed by the $K^{*}$ rest frame, the phase space factor for the cascade decay is

$$
\begin{aligned}
d \mathcal{P}_{B} & =\frac{1}{2 m_{B}} \frac{1}{(2 \pi)^{5}} \frac{d^{3} p_{\pi}}{2 E_{\pi}} \frac{d^{3} p_{K}}{2 E_{K}} \frac{d^{3} k}{2 E_{\gamma}} \delta^{4}\left(p_{B}-k-p_{K}-p_{\pi}\right) \\
& \rightarrow \frac{m_{K^{*}} p_{K \pi}}{\hat{s}^{1 / 2}} \frac{m_{B}^{2}-m_{K^{*}}^{2} \hat{s}}{32(2 \pi)^{4} m_{B}^{3}} d \Omega_{K} d \cos \theta_{\gamma} d \hat{s}
\end{aligned}
$$

performing all trivial integrals, including over the overall azimuthal orientation of the $K^{*}-\gamma-N$ plane. Similarly, for the BH conversion, computing in the lab frame,

$$
\begin{aligned}
d \mathcal{P}_{\mathrm{BH}} & =\frac{1}{2 M 2 E_{\gamma}} \frac{1}{(2 \pi)^{5}} \frac{d^{3} p_{+}}{2 E_{+}} \frac{d^{3} p_{-}}{2 E_{-}} \frac{d^{3} P^{\prime}}{2 E^{\prime}} \delta^{4}\left(P+k-P^{\prime}-p_{+}-p_{-}\right) \\
& \rightarrow\left[\frac{\mathcal{E}^{2}-\Delta^{2}}{64(2 \pi)^{5} M^{2} \mathcal{E}}\right] d \Omega_{+} d \Omega_{-} d \Delta
\end{aligned}
$$

Here the lepton momenta been approximated in the measure via $\sqrt{E_{ \pm}^{2}-m^{2}} \simeq E_{ \pm}$. We have further defined

$$
\mathcal{E} \equiv \frac{E_{+}+E_{-}}{2}, \quad \Delta \equiv \frac{E_{+}-E_{-}}{2}
$$

and enforced non-relativistic nuclear scattering, which implies $E^{\prime} \simeq M$ or equivalently $E_{\gamma} \simeq E_{+}+E_{-}$, up to $q^{0}=q^{2} / 2 M \ll m$ corrections. Hence to an excellent approximation $\mathcal{E}$ is half the photon energy in the lab frame. Moreover, note that $m \leq E_{ \pm} \leq E_{\gamma}$ implies 
that

$$
\Delta \in(m-\mathcal{E}, \mathcal{E}-m)
$$

At an $e^{+} e^{-} B$-factory, such as Belle or BaBar, the $e^{+} e^{-} \rightarrow \Upsilon \rightarrow B \bar{B}$ production factorizes from the subsequent $B$ decays, because the $B$ is a pseudoscalar. In this type of collider, the rapidity of parent $B$ meson in the lab frame has a known prior probability distribution, $f_{B}(\eta) d \eta$, determined by the collider configuration, and enters as an independent phase space factor in $d \mathcal{R}$. In Appendix D.II we include a derivation of the $B$ rapidity pdf (D.10), for an $e^{+} e^{-}$machine. We shall restrict ourselves hereafter to the case that $f_{B}(\eta)$ is known. In this case, note that the energy $\mathcal{E}$ is fully specified by $\eta, \hat{s}$ and $\theta_{\gamma}$, viz.

$$
\mathcal{E}\left(\eta, \theta_{\gamma}, \hat{s}\right) \equiv \frac{m_{B}^{2}-m_{K^{*}}^{2} \hat{s}}{4 m_{B}}\left(\cosh \eta+\cos \theta_{\gamma} \sinh \eta\right)
$$

so that the lepton energies can be expressed in terms of $\eta, \hat{s}, \theta_{\gamma}$ and $\Delta$, via eqs. (6.28). In our discussion of the kinematics above, it was convenient to express the amplitudes in terms of the ten phase space coordinates $\hat{s}, \theta_{\gamma}, E_{ \pm}, \Omega_{K}$ and $\Omega_{ \pm}$. We see here, however, that for the differential rate, it is more natural to choose $\eta$ and $\Delta$ as phase space coordinates rather than $E_{ \pm}$. Combining the above results together, the full differential rate

$d \mathcal{R}=|\mathcal{M}|^{2} f_{B}(\eta) \frac{m_{K^{*}} p_{K \pi}}{\hat{s}^{1 / 2}}\left[\frac{m_{B}^{2}-m_{K^{*}}^{2} \hat{s}}{4(4 \pi)^{9} m_{B}^{3}}\right]\left[\frac{\mathcal{E}^{2}\left(\eta, \theta_{\gamma}, \hat{s}\right)-\Delta^{2}}{M^{2} \mathcal{E}\left(\eta, \theta_{\gamma}, \hat{s}\right)}\right] d \Omega_{K} d \Omega_{+} d \Omega_{-} d \Delta d \cos \theta_{\gamma} d \hat{s} d \eta$ 


\subsubsection{Polarization and weak phase observables}

Let us now define a further change of azimuthal angular coordinates, modulo $2 \pi$

$$
\begin{aligned}
& \psi \equiv \phi_{+}+\phi_{-}+2 \phi_{K}, \\
& \bar{\psi} \equiv \phi_{+}+\phi_{-}-2 \phi_{K}, \\
& \varphi \equiv \phi_{+}-\phi_{-} .
\end{aligned}
$$

The angle $\varphi$ encodes the acoplanarity of the leptons with respect to the photon, with coplanarity corresponding to $\varphi=\pi$. Note that $\phi_{K}$ and $\phi_{ \pm}$are defined with opposite orientations around the photon momentum direction $(\hat{m}) k$ (see Fig. 6.1). For coplanar conversion leptons and a stationary $B$ in the lab frame, $\psi$ then corresponds to the relative twist between the positron-electron conversion plane and the $K-\pi$ decay plane. Similarly, $\bar{\psi}$ would then correspond to the averaged orientation of the positron-electron conversion plane and the $K-\pi$ decay plane with respect to the $K^{*}-\gamma$ decay plane.

From eq. (6.16), the momentum exchange has the form $q^{2} \propto 1+\zeta \cos \varphi$, with $\zeta<1$. It follows from eqs. (6.15), (6.23) and (6.31) that the differential rate can be written in the form

$$
\begin{aligned}
& d \mathcal{R}=\left(\sum_{k} a_{k} \cos ^{k}(\varphi)\right)\left[A_{1}+A_{2} \cos (\psi+\varphi-\phi-\delta)+\right. \\
& \left.A_{3} \cos (\psi-\varphi-\phi-\delta)+A_{4} \cos (\psi-\phi-\delta)\right],
\end{aligned}
$$

where $a_{k}$ and $A_{i}$ are purely functions of the phase space orthogonal to $\psi, \bar{\psi}$ and $\varphi$. That is, $a_{k}$ and $A_{i}$ are functions of $\hat{s}, \eta, \theta_{\gamma, K, \pm}$ and $\Delta$. Integrating over all phase space except $d \psi$, we see that the marginal differential rates for $B \rightarrow\left(K^{*} \rightarrow K^{+} \pi^{-}\right)\left(\gamma \stackrel{\mathrm{BH}}{\rightarrow} e^{+} e^{-}\right)$and 
$\bar{B} \rightarrow\left(\bar{K}^{*} \rightarrow K^{-} \pi^{+}\right)\left(\gamma \stackrel{\mathrm{BH}}{\rightarrow} e^{+} e^{-}\right)$must respectively have the form

$$
\begin{aligned}
\frac{d \mathcal{R}}{d \psi} & =\frac{\mathcal{R}}{2 \pi}\left[1-\frac{2 r}{1+r^{2}} \mathcal{C} \cos (\psi-\phi-\delta)\right], \\
\frac{d \overline{\mathcal{R}}}{d \psi} & =\frac{\overline{\mathcal{R}}}{2 \pi}\left[1-\frac{2 r}{1+r^{2}} \mathcal{C} \cos (\psi-\phi+\delta)\right] .
\end{aligned}
$$

Eqs. (6.23) tell us that the cosine coefficient $\mathcal{C}$ arises from a ratio of $\mathrm{BH}$ interference terms to the BH squared amplitude, and is therefore independent of $r$ or $\phi$. In other words, this coefficient is the same for both of the $\mathrm{CP}$ conjugate processes, and is $B \rightarrow$ $K^{*} \gamma$ operator independent. We have chosen the relative sign of $\mathcal{C}$ in eqs (6.34) to anticipate the choice that ensures $\mathcal{C}>0$. Further, we have chosen the normalization of $\mathcal{C}$ in eqs (6.34) to ensure, via positive semi-definiteness of $d \mathcal{R} / d \psi$, that $|\mathcal{C}| \leq 1$, noting that the $r$ dependent factor $2 r /\left(1+r^{2}\right) \leq 1$ for any $r$. The coefficient $\mathcal{C}$ may then be interpreted as the maximum possible ratio of the amplitude of $d \mathcal{R} / d \psi$ oscillations to their average value, $\mathcal{R} / 2 \pi$. Hereafter we call this ratio the relative oscillation amplitude.

Eqs (6.34) are the main results of this chapter. Once the model-independent coefficient $\mathcal{C}$ is computed, then measurement of the relative oscillation amplitude in $d \mathcal{R} / d \psi$ permits extraction of $r$ up to the two-fold ambiguity $r \leftrightarrow 1 / r$. Further, measurement of the average phase shift (phase shift difference) between $d \mathcal{R} / d \psi$ and $d \overline{\mathcal{R}} / d \psi$ permits extraction of the weak (strong) phase $\phi(\delta)$. Equivalently, one may construct two forward-backward type asymmetries. Defining the four quadrants I : $\psi \in[0, \pi / 2]$, II $: \psi \in[\pi / 2, \pi]$, III $: \psi \in[\pi, 3 \pi / 2]$ and IV $: \psi \in[3 \pi / 2,2 \pi]$ then

$$
\begin{aligned}
\Psi_{\psi} & \equiv \mathcal{R}^{-1} \int_{- \text {I-II+III+IV }} \frac{d \mathcal{R}}{d \psi} d \psi=\frac{2}{\pi} \frac{2 r}{1+r^{2}} \mathcal{C} \sin (\phi+\delta) \\
\Omega_{\psi} & \equiv \mathcal{R}^{-1} \int_{-\mathrm{I}+\mathrm{II}+\mathrm{III}-\mathrm{IV}} \frac{d \mathcal{R}}{d \psi} d \psi=\frac{2}{\pi} \frac{2 r}{1+r^{2}} \mathcal{C} \cos (\phi+\delta),
\end{aligned}
$$


and moreover

$$
\begin{aligned}
\bar{\Psi}_{\psi} & \equiv \overline{\mathcal{R}}^{-1} \int_{-\mathrm{I}-\mathrm{II}+\mathrm{III}+\mathrm{IV}} \frac{d \overline{\mathcal{R}}}{d \psi} d \psi=\frac{2}{\pi} \frac{2 r}{1+r^{2}} \mathcal{C} \sin (\phi-\delta) \\
\bar{\Omega}_{\psi} & \equiv \overline{\mathcal{R}}^{-1} \int_{-\mathrm{I}+\mathrm{II}+\mathrm{III}-\mathrm{IV}} \frac{d \overline{\mathcal{R}}}{d \psi} d \psi=\frac{2}{\pi} \frac{2 r}{1+r^{2}} \mathcal{C} \cos (\phi-\delta)
\end{aligned}
$$

Note that all four symmetries have an upper bound $2 / \pi$. For known $\mathcal{C}$, one may extract $r$ and $\phi \pm \delta$ from these two sets of asymmetries.

\subsubsection{Statistics and Sensitivity Enhancements}

Before proceeding to numerical computation of $\mathcal{C}$, let us pause to consider the statistical confidence in the extraction of $r$ and $\phi$. We focus on their extraction from the asymmetries (6.35). These asymmetries are expectation values of a random variable defined to take the values \pm 1 on the quadrants I, II, III and IV, as specified in eqs. (6.35). For a sample of $N \gg 1$ events, the corresponding error $\sigma_{X}=\sqrt{\left(1-X^{2}\right) / N} \simeq 1 / \sqrt{N}$, for $X=\Psi_{\psi}, \Omega_{\psi}, \bar{\Psi}_{\psi}$, and $\bar{\Omega}_{\psi}$. The statistical confidence at which one rejects the SM values $X_{\mathrm{SM}}$ - thus measuring NP effects - is then characterized by the chi-square statistic $\left(X-X_{\mathrm{SM}}\right)^{2} / \sigma_{X}^{2}$.

As shown in Ref. [299] and below, special 'sensitivity parameter' kinematic cuts may enhance $\mathcal{C}$ on the resulting remaining phase space. The construction of these cuts is motivated by the observation, from eqs. (6.23), that $\mathcal{C}$ is enhanced on those areas of phase space in which the $\mathrm{BH}$ interference term, $\sim \alpha_{12}^{-} \alpha_{21}^{-}$, is comparable or larger than terms in the total BH square amplitude $|\alpha|^{2}=\sum_{\lambda, r, s}\left|\alpha_{r s}^{\lambda}\right|^{2}$. For example, one may 
define the sensitivity parameter

$$
\begin{aligned}
\mathcal{S} & \equiv\left|\alpha_{12}^{-} \alpha_{21}^{-}\right| /\left|\alpha_{11}^{-}\right|^{2} \\
& \simeq 2\left(1-\cos \left[\left|\phi_{+}-\phi_{-}\right|-\pi\right]\right)\left[\frac{\gamma_{+} \gamma_{-}}{\left(\gamma_{+}+\gamma_{-}\right)^{2}}\right]\left[\frac{\gamma_{+} \theta_{+} \gamma_{-} \theta_{-}}{\left(\gamma_{+}^{2} \theta_{+}^{2}-\gamma_{-}^{2} \theta_{-}^{2}\right)^{2}}\right]\left(1+\gamma_{+}^{2} \theta_{+}^{2}\right)\left(1+\gamma_{-}^{2} \theta_{-}^{2}\right) .
\end{aligned}
$$

Requiring $\mathcal{S} \gtrsim 1$ produces an event-level kinematic cut that typically leads to $\mathcal{O}(1)$ enhancements of $\mathcal{C}$ on the remaining phase space, as will be verified below. Note that the $\left(1-\cos \left[\left|\phi_{+}-\phi_{-}\right|-\pi\right]\right)$ factor in eq. (6.36) implies that $\mathcal{S} \simeq 0$ for coplanar events, and moroever that a lower bound on $\mathcal{S}$ typically favors events with higher acoplanarity. One may also consider other sensitivity parameters, such as

$$
\mathcal{T} \equiv 2\left|\alpha_{12}^{-} \alpha_{21}^{-}\right| /|\alpha|^{2}
$$

which is normalized such that $\mathcal{T} \in[0,1]$.

Let us define $\mathcal{C}_{0}\left(N_{0}\right)$ to be the relative oscillation amplitude (number of events) in the absence of $\mathcal{S}$ or $\mathcal{T}$ cuts, and write

$$
\mathcal{C}_{\mathrm{c}} \equiv \mathcal{C}\left[\mathcal{S}>\mathcal{S}_{\mathrm{c}}, \mathcal{T}>\mathcal{T}_{\mathrm{c}}\right], \quad N_{\mathrm{c}} \equiv N\left[\mathcal{S}>\mathcal{S}_{\mathrm{c}}, \mathcal{T}>\mathcal{T}_{\mathrm{c}}\right]
$$

Compared to the $\mathcal{S}_{\mathrm{c}}=\mathcal{T}_{\mathrm{c}}=0$ case, the application of sensitivity parameter cuts scales the NP statistical confidence by the factor

$$
\left.\Sigma \equiv\left(\frac{X-X_{\mathrm{SM}}}{\sigma_{X}}\right)^{2}\right|_{\mathcal{S}>\mathcal{S}_{\mathrm{c}}, \mathcal{T}>\mathcal{T}_{\mathrm{c}}}\left(\frac{\sigma_{X}}{X-X_{\mathrm{SM}}}\right)^{2} \simeq\left(\frac{\mathcal{C}_{\mathrm{c}}}{\mathcal{C}_{0}}\right)^{2} \frac{N_{\mathrm{c}}}{N_{0}}
$$

for $X=\Psi_{\psi}, \Omega_{\psi}, \bar{\Psi}_{\psi}$, and $\bar{\Omega}_{\psi}$. That is, the enhancement $\mathcal{C}_{\mathrm{c}} / \mathcal{C}_{0}$ achieved by the sensitivity parameter cuts competes with the corresponding increase in the statistical error, $\sqrt{N_{0} / N_{\mathrm{c}}}$, since necessarily $N_{\mathrm{c}}<N_{0}$. We shall see in the next section that there are 
choices of $\mathcal{S}_{\mathrm{c}}$ and $\mathcal{T}_{\mathrm{c}}$ for which $\Sigma>1$. For the purpose of measuring NP effects, this is equivalent to an effective increase in the sample size $N_{0} \mapsto \Sigma N_{0}$ - an increase in the effective statistics.

\subsection{Simulations}

\subsubsection{Relative Oscillation Amplitude}

Extraction of the relative oscillation amplitude coefficient $\mathcal{C}$ is achieved numerically via Monte-Carlo (MC) generation of $B \rightarrow\left(K^{*} \rightarrow K^{+} \pi^{-}\right)\left(\gamma \stackrel{\mathrm{BH}}{\rightarrow} e^{+} e^{-}\right)$events according to the differential rate (6.31). We use the matrix element in eq. (D.4), generated from linearly polarized photon amplitudes. Though it does not provide the same analytical insight as the matrix element generated from spinor-helicity methods (6.23), this matrix element is as numerically stable as the latter, and moreover, acts as a convenient crosscheck of the analytic results in eqs. (6.23) and (6.34).

For the numerical results shown in this chapter, we use a private MC code written in C/Python. For simplicity, we apply the narrow $K^{*}$ limit (6.7), which fixes the $K^{*}$ to be on-shell. We assume the nominal Belle(II) parameters [302] (see also App. D.II) in order to determine the $B$ rapidity distribution $f_{B}(\eta)$, which is peaked at $\beta \gamma \simeq 0.29$. More details of the operation of this MC generator are included in Appendix D.III. We have further checked the numerical results with a second private $\mathrm{MC}$, written in $\mathrm{C}++^{+}$Java, that makes use of the matrix element (6.23). In both codes, we discard the overall normalization of the matrix element - e.g. $A(r)$ in eqs. (6.23) - since we are concerned only with the relative oscillation amplitude, $\sim \mathcal{C}$.

In order to account for limited angular resolution, we include hereafter cuts on the lepton polar angles, $\theta_{ \pm}$, and opening angle, $\theta_{\ell \ell}$, defined in Fig. 6.1. We will consider a 

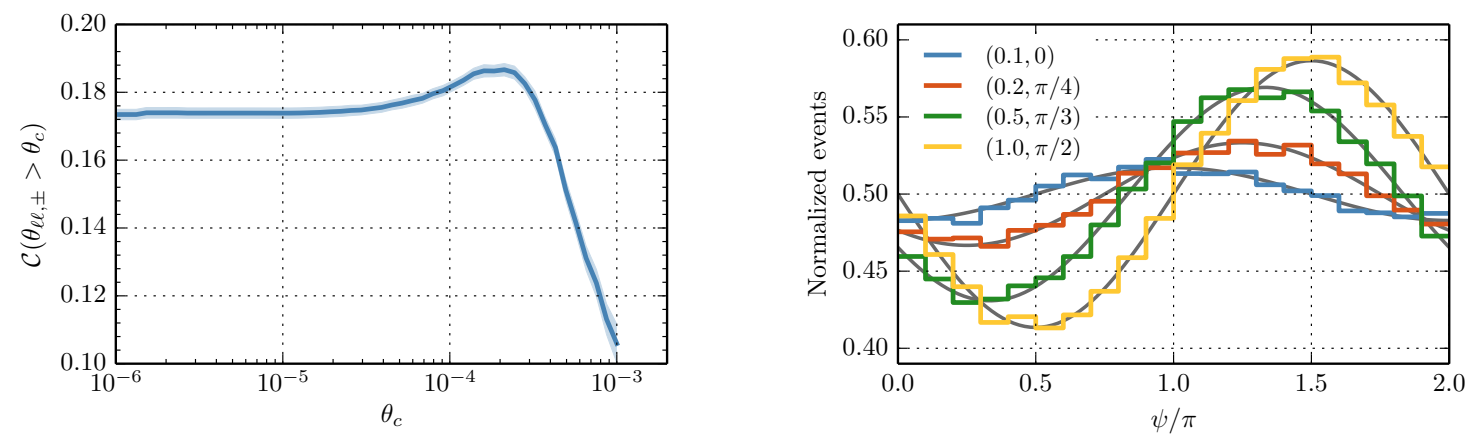

Figure 6.2: Left: The fit value for $\mathcal{C}$ with the $\pm 1 \sigma$ error band as a function of the polar angle cuts $\theta_{\ell \ell, \pm}>\theta_{\mathrm{c}}$ (see Fig. 6.1). The peak value of $\mathcal{C}$ approximately coincides with the peak of the $\theta_{ \pm}$marginal distribution (see the left panel of Fig. D.2). Right: Normalized differential distribution $d \mathcal{R} / d \psi$ for four different $(r, \phi+\delta)$ couplets and $\theta_{\mathrm{c}}=10^{-6}$. Also shown are theory predictions (gray) for the input values of $(r, \phi+\delta)$ and the extracted value $\mathcal{C}\left[\theta_{\mathrm{c}}=10^{-6}\right]$ in eq. (6.41).

uniform polar cut

$$
\theta_{\ell \ell, \pm}>\theta_{\mathrm{c}}
$$

for various values of $\theta_{c}$. In particular, we consider two benchmark cases $\theta_{c}=10^{-6}$ and $5 \times 10^{-4}$. The former captures almost all conversion leptons in the $B \rightarrow\left(K^{*} \rightarrow\right.$ $\left.K^{+} \pi^{-}\right)\left(\gamma \stackrel{\mathrm{BH}}{\rightarrow} e^{+} e^{-}\right)$process for semirelativistic $B^{\prime}$ s, while the latter might be plausibly achievable in the near- to mid-term future. To extract $\mathcal{C}$, we fit eq. (6.34) to the $d \mathcal{R} / d \psi$ histograms for various choices of $r$ and $\phi+\delta$, including the couplets $\{r, \phi+\delta\}=$ $\{(0.1,0),(0.2, \pi / 4),(0.5, \pi / 3),(1.0, \pi / 2)\}$. In Fig. 6.2 we show the extracted $\mathcal{C}$ as a function of the $\theta_{c}$ cuts. The maximal relative oscillation amplitude one can expect is of $\mathcal{O}(20 \%)$, and the benchmark extracted $\mathcal{C}$ values are

$$
\mathcal{C}\left[\theta_{\mathrm{c}}=10^{-6}\right]=0.173 \pm 0.001, \quad \text { and } \quad \mathcal{C}\left[\theta_{\mathrm{c}}=5 \times 10^{-4}\right]=0.150 \pm 0.003
$$

where the errors are purely statistical in origin. We also show in Fig. 6.2 typical $d \mathcal{R} / d \psi$ histograms for various choices of $r$ and $\phi+\delta$. The expected shifted and amplitudemodulated cosine can be clearly seen. Applying the extracted value for $\mathcal{C}$, these his- 


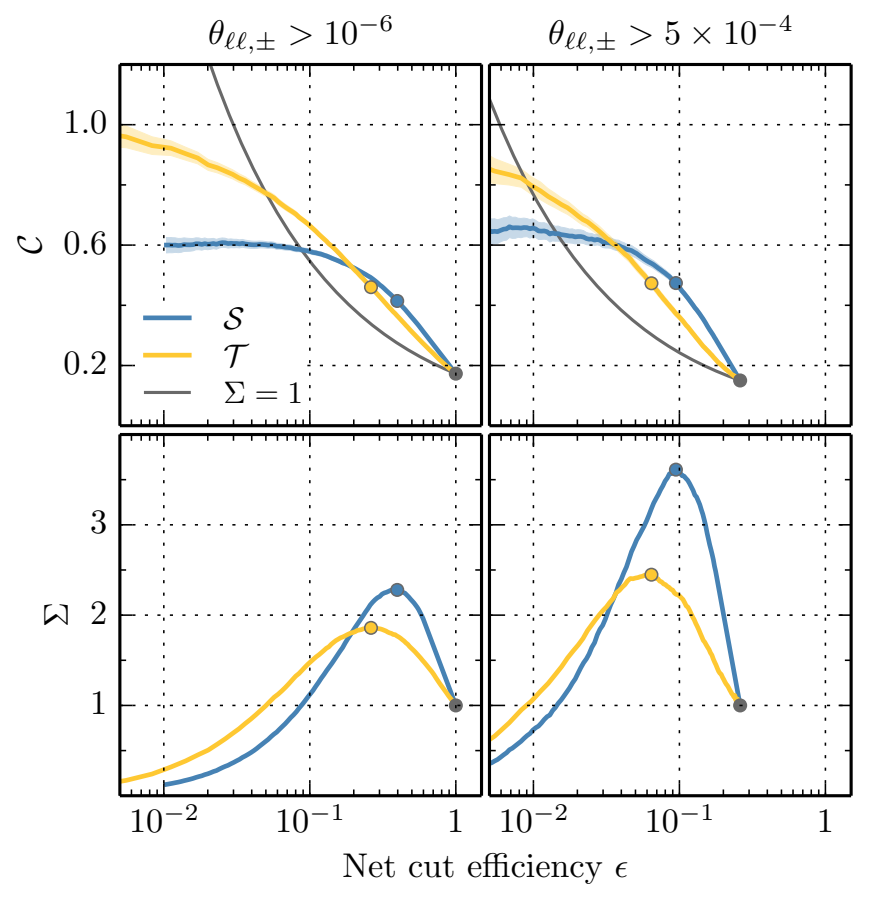

Figure 6.3: Upper panels: The coefficient $\mathcal{C}$ as a function of the cut efficiency for the $\mathcal{S}$ (blue) and $\mathcal{T}$ (gold) kinematic cuts, with polar cuts $\theta_{\mathrm{c}}=10^{-6}$ (left) and $\theta_{\mathrm{c}}=5 \times 10^{-4}$ (right). The colored regions depict the $\pm 2 \sigma$ statistical error bands. The equivalent effective statistics curve $\Sigma=1$, i.e. $\mathcal{C}=\mathcal{C}_{0} / \sqrt{N / N_{0}}$, is also shown (gray). Lower panels: Statistics enhancement $\Sigma$ as a function of the cut efficiency. The maxima correspond to the optimum cuts $\mathcal{S}>\mathcal{S}_{\mathrm{c}}^{\mathrm{opt}}$ and $\mathcal{T}>\mathcal{T}_{\mathrm{c}}^{\mathrm{opt}}$ (colored dots in all panels).

tograms are in excellent agreement with the theory predictions (6.34).

\subsubsection{Statistics Enhancements}

Incorporating the $\mathcal{S}$ and $\mathcal{T}$ kinematic cuts, we show in Fig. 6.3 the absolute enhancement of $\mathcal{C}$ as a function of the net cut efficiency, $\epsilon$, for the two benchmark polar angle cuts. The net cut efficiency is defined hereafter to be the fraction of events kept after application of both kinematic and polar cuts. The pure $\mathcal{S}$ cuts - that is, $\mathcal{C}\left(\mathcal{S}>\mathcal{S}_{\mathrm{c}}, \mathcal{T}>0\right)$ - provide the larger enhancement at high cut efficiencies. At lower efficiencies the pure $\mathcal{T}$ cut provides the larger enhancement. The $\mathcal{C}$ and $\epsilon$ dependence on $\mathcal{S}_{\mathrm{c}}$ may be read off from Fig. 6.4. 


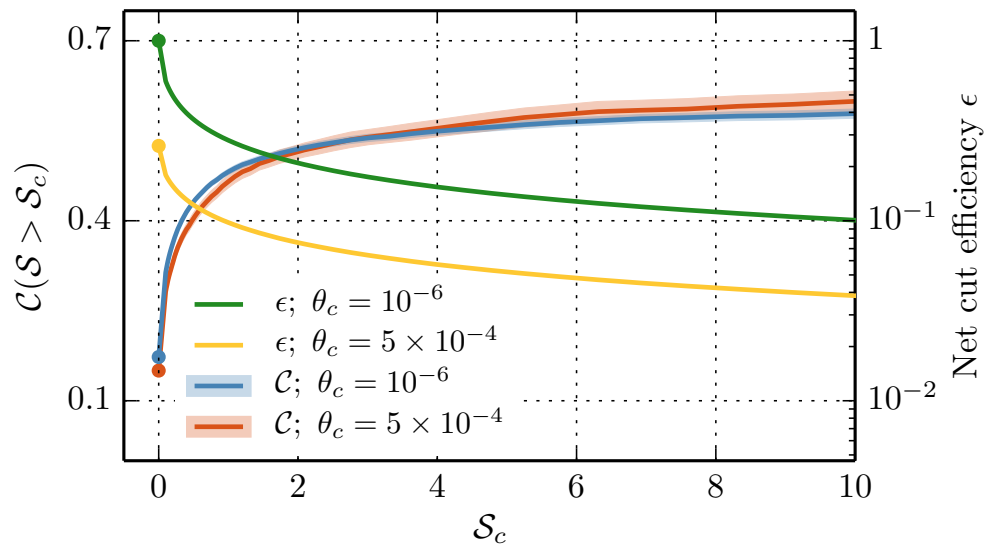

Figure 6.4: The enhancement in $\mathcal{C}$ as a function of $\mathcal{S}$. The secondary $y$-axis shows the corresponding cut efficiency. The colored regions depict the $\pm 2 \sigma$ statistical error bands.

Comparing to the equivalent effective statistics curve $\Sigma=1$ - i.e. $\mathcal{C}=\mathcal{C}_{0} / \sqrt{N / N_{0}}$ - we see in Fig. 6.3 that the $\mathcal{C}$ dependence initially rises faster than $\mathcal{C}_{0} / \sqrt{N / N_{0}}$. This means that for low values of $\mathcal{S}_{\mathrm{c}}$ and $\mathcal{T}_{\mathrm{c}}$, the statistics on the $\mathcal{S}$ and $\mathcal{T}$ cut phase space is enhanced compared to the full data set. The explicit $\Sigma$ dependence is also shown in Fig. 6.3. In particular, one sees that for $\theta_{c}=5 \times 10^{-4}$, the statistical enhancement $\Sigma$ is optimized at $\mathcal{S}_{\mathrm{c}}^{\mathrm{opt}}=1.1\left(\mathcal{T}_{\mathrm{c}}^{\mathrm{opt}}=0.62\right)$ corresponding to $\Sigma=3.6(\Sigma=2.5)$.

To demonstrate the efficacy of these statistics enhancements, in Table 6.1 we compute the extracted values for $r$ and $\phi+\delta$, along with their statistical errors, with and without the optimal $\mathcal{S}_{\mathrm{c}}^{\text {opt }}$ and $\mathcal{T}_{\mathrm{c}}^{\text {opt }}$ cuts. We use an MC sample generated from an input couplet $(r, \phi+\delta)=(0.2, \pi / 4)$, containing a total of $N=10^{4}$ events. This roughly corresponds to $50 \mathrm{ab}^{-1}$ of data - a benchmark luminosity at Belle II [302] - and a percent level photon conversion rate with ideal acceptance efficiency. The polar cut is $\theta_{\mathrm{c}}=5 \times 10^{-4}$. The extracted values are obtained by two different methods, first from a fit to the differential rate eq. (6.34) and second from the quadrant asymmetries (6.35).

The statistical errors for the optimized kinematic $\mathcal{S}$ and $\mathcal{T}$ cuts are a $\mathcal{O}(1)$ factor smaller than for the full data set, as expected from the above numerical analysis: Application of these sensitivity parameter cuts improves the statistical power of the $r$ and 


\begin{tabular}{lcccccc}
\hline \hline Method & $r^{2} \mathcal{S}, \mathcal{T}>0$ & \multicolumn{2}{c}{$\mathcal{S}>\mathcal{S}_{\mathrm{c}}^{\text {opt }}$} & \multicolumn{2}{c}{$\mathcal{T}>\mathcal{T}_{\mathrm{c}}^{\text {opt }}$} \\
\hline \multirow{2}{*}{$/ d \psi$} & $0.194 \pm$ & $0.255 \pm$ & $0.194 \pm$ & $0.263 \pm$ & $0.190 \pm$ & $0.247 \pm$ \\
& 0.017 & 0.026 & 0.010 & 0.015 & 0.013 & 0.020 \\
\multirow{2}{*}{$\Psi_{\psi}, \Omega_{\psi}$} & $0.217 \pm$ & $0.216 \pm$ & $0.203 \pm$ & $0.248 \pm$ & $0.192 \pm$ & $0.232 \pm$ \\
& 0.005 & 0.157 & 0.003 & 0.088 & 0.003 & 0.112 \\
\hline \hline
\end{tabular}

Table 6.1: Extracted values of $r$ and $(\phi+\delta)$ from an MC sample with input values $(0.2, \pi / 4)$.

$\phi$ extraction from $B \rightarrow\left(K^{*} \rightarrow K^{+} \pi^{-}\right)\left(\gamma \stackrel{\mathrm{BH}}{\rightarrow} e^{+} e^{-}\right)$events. Moreover, even without these enhancements, both $r$ and $\phi$ are extracted with sufficient numerical precision to probe NP effects at the $r \sim 1$ or $\phi \sim 1$ level.

\subsection{Chapter summary}

In this chapter we have presented the helicity amplitudes and differential rate for the $B \rightarrow K^{*} \gamma \rightarrow K^{+} \pi^{-} \gamma$ process, in which the photon undergoes subsequent nuclear conversion to a lepton pair. Interference between the intermediate, on-shell photon polarizations in the coherent $B \rightarrow\left(K^{*} \rightarrow K^{+} \pi^{-}\right)\left(\gamma \stackrel{\mathrm{BH}}{\rightarrow} e^{+} e^{-}\right)$process produces oscillations in the angular kinematic observable $\psi$. Measuring the amplitude and phase of these oscillations - or equivalently two quadrant-type asymmetries $\Psi_{\psi}$ and $\Omega_{\psi}$-permits extraction in principle of the polarization ratio, $r$, and the relative weak phase, $\phi$, of the right- and left-handed $B \rightarrow K^{*} \gamma$ amplitudes. In this manner, SM expectations for both $r$ and $\phi$ may be tested.

We have employed private Monte Carlo simulations to compute the $\psi$ distribution and asymmetries as a function of $r, \phi$ and kinematic cuts. In particular, kinematic cuts with respect to the sensitivity parameters $\mathcal{S}$ and $\mathcal{T}$ may sufficiently amplify these oscillations, such that the overall statistical power of the $r$ and $\phi$ measurement is increased by an $\mathcal{O}(1)$ factor.

Implementing this approach using converted photons will be experimentally chal- 
lenging, not least because of the high angular resolution required to reconstruct the conversion lepton kinematics. Moreover, a detector whose thickness is on the order of one radiation length or less is required to avoid multiple leptonic rescatterings, that otherwise smear the lepton kinematics. Nonetheless, the theoretically clean nature of the $r$ - and $\phi$-sensitive observables presented in this work may perhaps encourage the use of this technique in a future dedicated detector element. 


\section{Chapter 7}

\section{Conclusion}

While the standard model of particle physics has successfully withstood many experimental tests, it still leaves many questions unanswered. Attempting to answer these questions motivates one to propose physics beyond the standard model. Moreover, possible solutions to the hierarchy problem in addition to the WIMP miracle single out the $\mathrm{TeV}$ scale as the energy scale of new physics. In the first part of this thesis, we explored three different dark matter models where both the dark matter candidate and the mediators between the visible and dark sectors are at the $\mathrm{TeV}$ scale. While, in the second part, we were motivated by the matter/antimatter asymmetry to look for new sources of $\mathrm{CP}$ violation. We focused on developing tools for probing $\mathrm{CP}$ violation in the Higgs and flavor sectors.

Starting with DM model building, we exploited the flavor symmetries of the standard model to stabilize a dark matter candidate using two different approaches. In chapter 2, we showed that exploiting the flavor structure of the SM can lead to metastable $\mathrm{DM}$ with $\mathrm{TeV}$ scale mediators even if the DM itself is flavor neutral. We did this in two different flavor breaking scenarios: in the context of minimal flavor violation and in a Froggatt-Nielsen flavor model. In chapter 3, we turned our attention to flavored dark matter in a fully gauged $S U(3)^{3}$ flavor model. We took advantage of a remnant 
$Z_{3}$ symmetry that is useful to stabilize a dark matter that carries a flavor charge and investigated its phenomenology. We then showed that, in the case of a fermionic dark matter candidate where the only source of splitting in the multiplet is from radiative corrections involving the flavor gauge bosons, the dark matter mass must lie in the range $[0.5,5] \mathrm{TeV}$ to satisfy all experimental constraints.

On a more speculative note, in chapter 4 we investigated the possibility of obtaining bounds on the up, down, and strange quark Yukawa couplings if Higgs portal dark matter is discovered. Surprisingly, we found that while in the limit where the light Yukawas tend to zero there is virtually no effect on the direct detection rate while, in the limit where they are allowed to saturate the current experimental bounds, the direct detection rate can be enhanced by four orders of magnitude. We then reviewed a range of flavor models to determine the expected enhancement or suppression of the light Yukawa couplings in realistic model building scenarios.

Finally, we developed techniques for measuring CP violation in the Higgs coupling to photons in chapter 5 and in rare radiative decays of $B$ mesons in chapter 6 . We found that while these techniques provide a theoretically clean probe of CPV, they require specialized detectors in order to be practical.

In summary, the standard model does not provide answers for well motivated questions including the microscopic theory of dark matter and the source of the matter/antimatter asymmetry. We attempted to address the aforementioned questions in this thesis. However, the topics considered here are too rich for one thesis and provide a solid basis for an ongoing exploration. 


\section{A - Appendix to Chapter 2}

\section{A.I Operators in four component notation}

In the chapter we are using a two-component notation, where the left-handed Weyl fermion fields $\left(q_{i}, u^{c}, d^{c}, l_{i}, e^{c}\right)$ have hypercharges $(+1 / 6,-2 / 3,+1 / 3,-1 / 2,+1)$ and $B-$ $L$ charges $(1 / 3,-1 / 3,-1 / 3,-1,+1)$. The higgs doublet is denoted by $H$ and has $Y=$ $+1 / 2$, while $\tilde{H}=i \sigma_{2} H^{*}$. The two Weyl spinors of the DM Dirac fermion are $\psi$ and $\psi^{c}$ with $B-L=-1$ and +1 , respectively. Finally, $\phi$ is the complex scalar DM with $B-L=2$. Capital letters denote four-component spinors following the notation in [305]. The DM Dirac fermion $\Psi$ and its charge conjugate $\Psi^{C}$ are

$$
\Psi=\left(\begin{array}{c}
\psi_{\alpha} \\
\psi^{c \dagger \dot{\alpha}}
\end{array}\right), \quad \Psi^{C}=\left(\begin{array}{c}
\psi_{\alpha}^{c} \\
\psi^{\dagger \dot{\alpha}}
\end{array}\right)
$$

while $\bar{\Psi}=\left(\psi^{c \alpha}, \psi_{\dot{\alpha}}\right)$. Writing for the two-component spinors $q_{1}=u_{L}, q_{2}=d_{L}$ and $\ell_{1}=\nu_{L}, \ell_{2}=e_{L}$, suppressing generation indices, we introduce

$$
U=\left(\begin{array}{c}
u_{L \alpha} \\
u^{c \dagger \dot{\alpha}}
\end{array}\right), \quad D=\left(\begin{array}{c}
d_{L \alpha} \\
d^{c \dagger \dot{\alpha}}
\end{array}\right), \quad E=\left(\begin{array}{c}
e_{L \alpha} \\
e^{c \dagger \dot{\alpha}}
\end{array}\right), \quad N=\left(\begin{array}{c}
\nu_{L \alpha} \\
\nu^{c \dagger \dot{\alpha}}
\end{array}\right)
$$


where $\nu^{c}$ is the right-handed neutrino field introduced for completeness. If neutrino is Majorana, $\nu_{L}=\nu^{c}$. The weak doublets in the four-component notation are

$$
Q_{L}=\left(U_{L}, D_{L}\right), \quad L_{L}=\left(N_{L}, E_{L}\right)
$$

with $U_{L} \equiv P_{L} U$, etc, and similarly $U_{R} \equiv P_{R} U, D_{R} \equiv P_{R} D, \ldots$. Some examples of the relevant asymmetric operators in the two- and four-component notations are given below.

$$
\begin{array}{ll}
\operatorname{dim} \text { 6: } & \mathcal{Q}_{1}^{(6)}=\left(q_{i} q^{i}\right)\left(d^{c *} \psi^{c *}\right)=\left(\overline{Q_{L i}^{C}} Q_{L}^{i}\right)\left(\overline{D^{C}} P_{R} \Psi\right), \\
& \mathcal{Q}_{2}^{(6)}=\left(u^{c} d^{c}\right)\left(d^{c} \psi^{c}\right)=\left(\bar{U} P_{L} D^{C}\right)\left(\bar{D} P_{L} \Psi^{C}\right),
\end{array}
$$

$\underline{\operatorname{dim} 10:} \quad \mathcal{Q}_{1}^{(10)}=\phi\left(d^{c} d^{c}\right)\left(q_{i}^{*} q^{i *}\right)\left(q_{j}^{*} q^{j *}\right)=\phi\left(\bar{D} P_{L} D^{C}\right)\left(\overline{Q_{L i}} P_{R} Q_{L}^{C i}\right)\left(\overline{Q_{L i}} P_{R} Q_{L}^{C i}\right)$.

\section{A.II Asymmetric DM relic density}

Here we review the relations between the DM relic density and the DM mass in ADM models. We assume that the operator(s) transferring the $B-L$ asymmetry from the visible to the dark sector decouple above electroweak phase transition, $T_{C}>T_{\mathrm{ew}} \sim 170$ $\mathrm{GeV}$ [306], as is the case for our ADM models, see Sec. 2.2. We first assume that the visible sector consists below $T_{C}$ of only the SM fields (we will later relax this). The number density asymmetry for relativistic particles is

$$
(n-\bar{n})_{i}=\frac{T^{3}}{6} \hat{g}_{i} \frac{\mu_{i}}{T}
$$

where $n(\bar{n})$ are the particle(anti-particle) number densities, $\mu_{i}$ is the chemical potential for species $i$, and $\hat{g}_{i}=g_{i}\left(g_{i} / 2\right)$ for bosons (fermions) with $g_{i}$ internal degrees of freedom so that $\hat{g}_{i}=1$ for a Weyl fermion, while $\hat{g}_{i}=2$ for a Dirac fermion or a complex scalar. 
All the SM particles are in chemical equilibrium, so that the chemical potentials are proportional to the conserved quantum numbers [307]. Above the electroweak phase transition these are $B-L, Y$ and $S U(2)_{L}$, while $B+L$ is broken by sphalerons. Thus (see also [308])

$$
\mu_{i}=\left(T_{3}\right)_{i} c_{3}+Y_{i} c_{Y}+(B-L)_{i} c_{B-L}
$$

where the $c_{i}$ are constants that we determine from net weak isospin, hypercharge and $B-L$ densities. The net weak isospin charge density in the universe normalized to entropy density is

$$
T_{3} \propto \sum_{i} \hat{g}_{i}\left(T_{3}\right)_{i} \mu_{i}=\sum_{i} \hat{g}_{i}\left(T_{3}\right)_{i}^{2} c_{3}+0 \cdot c_{Y}+0 \cdot c_{B-L}=0
$$

For the first equality we used that for each $\mathrm{SU}(2)$ multiplet $\sum_{i}\left(T_{3}\right)_{i}=0$, and in the second equality that the net $T_{3}$ charge is zero since $S U(2)_{L}$ is not explicitly broken. Thus $c_{3}=0$ and the $S U(2)_{L}$ charge of a particle does not contribute to its chemical potential.

Flavor mixing ensures that the chemical potentials for SM Weyl fermions from different generations are the same. Similarly, $S U(2)_{L}$ interactions ensure that $\mu_{u_{L}}=\mu_{d_{L}} \equiv$ $\mu_{Q}$, and $\mu_{\ell_{L}}=\mu_{\nu} \equiv \mu_{L}$. We thus have

$$
\begin{gathered}
\mu_{H}=\frac{1}{2} c_{Y}, \quad \mu_{L}=-\frac{1}{2} c_{Y}-c_{B-L}, \quad \mu_{E}=-c_{Y}-c_{B-L}, \\
\mu_{Q}=\frac{1}{6} c_{Y}+\frac{1}{3} c_{B-L}, \mu_{U}=\frac{2}{3} c_{Y}+\frac{1}{3} c_{B-L}, \mu_{D}=-\frac{1}{3} c_{Y}+\frac{1}{3} c_{B-L},
\end{gathered}
$$

while for the gauge bosons $\mu_{G}=\mu_{W}=\mu_{B}=0$. The net hypercharge of the universe is 
thus

$$
\begin{aligned}
Y & \propto \sum_{i} \hat{g}_{i}(Y)_{i} \mu_{i}=\frac{1}{2} 2 \cdot 2 \mu_{H}+N_{f}\left[-\frac{1}{2} 2 \mu_{L}-\mu_{E}+N_{c}\left(\frac{1}{6} \cdot 2 \mu_{Q}+\frac{2}{3} \mu_{U}-\frac{1}{3} \mu_{D}\right)\right] \\
& =2 \mu_{H}+N_{f}\left(\mu_{Q}+2 \mu_{U}-\mu_{D}-\mu_{L}-\mu_{E}\right)=11 c_{Y}+8 c_{B-L},
\end{aligned}
$$

where $N_{f}=3$ is the number of generations and $N_{c}$ is the number of colors. Setting the net hypercharge density in the universe to zero, $Y=0$, gives

$$
c_{Y}=-\frac{8}{11} c_{B-L} .
$$

The net $B-L$ number density in the visible sector (i.e. excluding the $B-L$ asymmetry carried by the $\chi$ fields in the dark sector) is then

$$
B-L \propto N_{f}\left(-2 \mu_{L}-\mu_{E}+2 \mu_{Q}+\mu_{U}+\mu_{D}\right)=\frac{79}{11} c_{B-L} .
$$

There are two types of interactions between the dark and visible sector: the asymmetric interactions that involve a single $\chi$ field, and the symmetric interactions of the form $\chi^{\dagger} \chi$ times the SM fields. The symmetric operators keep the dark and the visible sectors in thermal equilibrium. The asymmetric interactions are suppressed, and decouple at temperatures well above the $\chi$ mass. At lower temperatures the $\chi$ number is thus effectively conserved. The chemical potential $\mu_{\chi}$ is the same as it was before the decoupling. We thus have

$$
\mu_{\chi}^{i}=(B-L)_{\chi}^{i} c_{B-L}
$$

where $(B-L)_{\chi}^{i}$ is the $B-L$ charge of the $\chi^{i}$ field. Here we allow for several $\chi^{i}$ fields in the dark sector and also define the weighted $B-L$ charge of the dark sector fields 
as

$$
(B-L)_{\chi}^{\mathrm{sum}} \equiv \sum_{i} \hat{g}_{\chi}^{i}(B-L)_{\chi}^{i}
$$

The net $\chi$ number density normalized to entropy density we denote by $\Delta \chi$ and is

$$
\Delta \chi \propto \sum_{i} \hat{g}_{\chi}^{i} \mu_{\chi}^{i}=(B-L)_{\chi}^{\operatorname{sum}} c_{B-L}
$$

Since $B-L$ and $\chi$ are conserved quantum numbers below the decoupling temperature, each of the number densities scales as $R^{-3}$ as universe expands. The ratio

$$
\frac{\Delta \chi}{B-L}=\left.\frac{\Delta \chi}{B-L}\right|_{\text {decoup. }}=\frac{11}{79}(B-L)_{\chi}^{\text {sum }}
$$

thus stays fixed.

Even if at the decoupling there are more $\chi_{i}$ dark sector states, we assume that DM is composed only from one state, $\chi$. We therefore have for the ratio of baryon and dark matter energy densities

$$
\frac{\Omega_{B}}{\Omega_{\chi}}=\frac{m_{p}}{m_{\chi}} \frac{B}{B-L} \frac{B-L}{\Delta \chi} .
$$

The ratio of net $B$ and $B-L$ numbers $B /(B-L)=28 / 79=0.354$ just above the electroweak phase transition [307]. This remains essentially unchanged even if sphaleron and top mass effects are taken into account, in which case using results from [306,309] one has $B /(B-L)=0.349$ for both scalar and fermionic DM. Using $(B-L) / \Delta \chi=$ $79 /\left(11(B-L)_{\chi}^{\text {sum }}\right)$ from (A.17) finally leads to

$$
m_{\chi}=2.509 m_{p} \frac{\Omega_{\chi}}{\Omega_{B}} \frac{1}{(B-L)_{\chi}^{\text {sum }}}=(12.5 \pm 0.8) \mathrm{GeV} \frac{1}{(B-L)_{\chi}^{\text {sum }}}
$$

where in the last equality we used $\Omega_{\chi}=0.265 \pm 0.011$ and $\Omega_{B}=0.0499 \pm 0.0022$ [55]. Note that the error is dominated by the experimental determination of DM and baryon densities. For instance, the difference between $B /(B-L)$ determination with and 
without sphaleron effects leads to a smaller shift in $m_{\chi}$ than the above quoted error.

We turn next to the case of additional fields in the visible sector. An example would be that SM gets completed to the MSSM. The relation between $Y, B-L$ and the constants $c_{Y, B-L}$ can be written in the matrix form

$$
\begin{aligned}
\left(\begin{array}{c}
Y \\
B-L
\end{array}\right) & =\frac{15}{4 \pi^{2} g_{*} T}\left(\begin{array}{cc}
\sum_{i} \hat{g}_{i} Y_{i}^{2} & \sum_{i} \hat{g}_{i} Y_{i}(B-L)_{i} \\
\sum_{i} \hat{g}_{i} Y_{i}(B-L)_{i} & \sum_{i} \hat{g}_{i}(B-L)_{i}^{2}
\end{array}\right) \cdot\left(\begin{array}{c}
c_{Y} \\
c_{B-L}
\end{array}\right) \\
& =\frac{15}{4 \pi^{2} g_{*} T}\left(\begin{array}{cc}
11+\left[Y^{2}\right]_{\mathrm{NP}} & 8+[Y(B-L)]_{\mathrm{NP}} \\
8+[Y(B-L)]_{\mathrm{NP}} & 13+\left[(B-L)^{2}\right]_{\mathrm{NP}}
\end{array}\right) \cdot\left(\begin{array}{c}
c_{Y} \\
c_{B-L}
\end{array}\right) .
\end{aligned}
$$

Here we defined

$$
\left[Y^{2}\right]_{\mathrm{NP}}=\sum_{i} \hat{g}_{i} Y_{i}^{2}, \quad[Y(B-L)]_{\mathrm{NP}}=\sum_{i} \hat{g}_{i} Y_{i}(B-L)_{i}, \quad\left[(B-L)^{2}\right]_{\mathrm{NP}}=\sum_{i} \hat{g}_{i}(B-L)_{i}^{2},
$$

where the sums run over the new states only. The solution for $B-L$ in terms of $c_{B-L}$ is obtained by solving the above matrix equation setting $Y=0$, from which

$$
B-L=\frac{15 c_{B-L}}{4 \pi^{2} g_{*} T}\left(13+\left[(B-L)^{2}\right]_{\mathrm{NP}}-\frac{\left(8+[Y(B-L)]_{\mathrm{NP}}\right)^{2}}{11+\left[Y^{2}\right]_{\mathrm{NP}}}\right) .
$$

The net $\chi$ charge is still given by Eq. (A.17), while the ratio $\Omega_{B} / \Omega_{\chi}$ is given by (A.18) with $(B-L) / \Delta \chi$ fixed at the decoupling temperature and $B /(B-L)$ at the electroweak phase transition. We thus have

$$
m_{\chi}=m_{p} \frac{\Omega_{\chi}}{\Omega_{B}} \frac{B}{B-L}\left(13+\left[(B-L)^{2}\right]_{\mathrm{NP}}-\frac{\left(8+[Y(B-L)]_{\mathrm{NP}}\right)^{2}}{\left(11+\left[Y^{2}\right]_{\mathrm{NP}}\right)^{2}}\right) \frac{1}{(B-L)_{\chi}^{\mathrm{sum}}},
$$

where $B /(B-L)=0.349$ and $(B-L)_{\chi}^{\text {sum }}$ given in (A.15). 


\section{A.III Calculation of the DM decay time}

Here we give further details of the DM lifetime calculation in the MFV and FN models for $B=2 \mathrm{DM}$, Sec. 2.3, while also varying the DM mass. The results are shown in Fig. 2.3. There are three different types of dimension 10 operators that can lead to DM decay, of schematic form $\chi\left(d^{c} d^{c}\right)\left(d^{c} d^{c}\right)\left(u^{c} u^{c}\right), \chi\left(d^{c} d^{c}\right)\left(d^{c} u^{c}\right)\left(q^{*} q^{*}\right)$, and $\chi\left(q^{*} q^{*}\right)\left(q^{*} q^{*}\right)\left(d^{c} d^{c}\right)$. For the same NP suppression scale $\Lambda$ the last type of operators gives the shortest lifetime. The dominant effective decay Lagrangian is thus, schematically,

$$
\mathcal{L}_{\text {dec }}^{(B=2)} \supset \frac{\mathcal{C}}{\Lambda^{6}} \chi\left(q^{*} q^{*}\right)\left(q^{*} q^{*}\right)\left(d^{c} d^{c}\right)
$$

where $\mathcal{C}$ is a flavor-dependent Wilson coefficient, the brackets enclose Lorentz contracted pairs, and summation over different flavor, color and weak isospin contractions is understood.

In Sec. 2.3 we included the SM Yukawa insertions in the definition of the operators. To unify the notation we instead use in this appendix the convention that the Wilson coefficient $\mathcal{C}$ encodes all the flavor suppressions. The effective decay Lagrangian is thus, going to the mass basis, and displaying the flavor indices only,

$$
\mathcal{L}_{\text {dec. }}^{(B=2)} \supset \frac{\mathcal{C}^{\mathrm{IJBCEF}}}{\Lambda^{6}} \chi u_{\mathrm{I}}^{*} u_{\mathrm{J}}^{*} d_{\mathrm{B}}^{*} d_{\mathrm{E}}^{*} d_{\mathrm{C}}^{c} d_{\mathrm{F}}^{c},
$$

where the flavor dependent Wilson coefficients are

$$
\begin{aligned}
& \mathcal{C}_{(\mathrm{MFV})}^{\mathrm{IJBCEF}} \simeq\left[V_{\mathrm{CKM}}\right]_{\mathrm{IA}}\left[Y_{d}^{\mathrm{diag}}\right]_{\mathrm{B}}\left[V_{\mathrm{CKM}}\right]_{\mathrm{JD}} \varepsilon^{\mathrm{ABC}} \varepsilon^{\mathrm{DEF}}, \\
& \mathcal{C}_{(\mathrm{FN})}^{\mathrm{IIBCEF}} \simeq \lambda^{\left|-H\left(q_{\mathrm{I}}\right)-H\left(q_{\mathrm{J}}\right)-H\left(q_{\mathrm{B}}\right)-H\left(q_{\mathrm{E}}\right)+H\left(d_{\mathrm{C}}^{c}\right)+H\left(d_{\mathrm{F}}^{c}\right)\right|},
\end{aligned}
$$



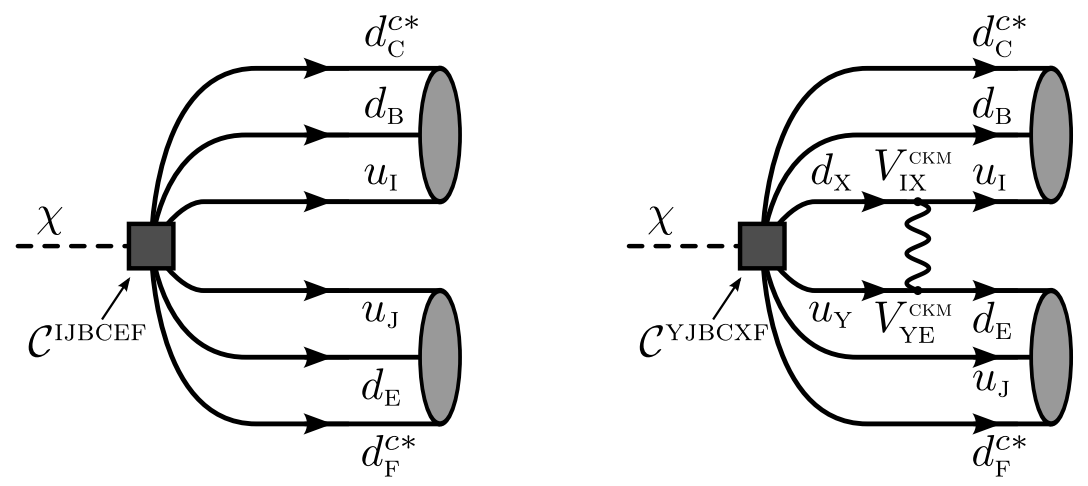

Figure A.1: Example Feynman diagrams for the decay of $B=2$ DM. The diagram on the left shows the tree level decay whereas the one on the right shows the loop-induced decay.

The partial decay width for $\chi \rightarrow q q q q d d$ transition is then, using NDA,

$$
\Gamma_{\chi} \simeq \frac{\mathcal{C}^{2}}{8 \pi} \frac{1}{\left(16 \pi^{2}\right)^{4}}\left(\frac{m_{\chi}}{\Lambda}\right)^{12} m_{\chi} .
$$

The factor $1 /(8 \pi) \times 1 /\left(16 \pi^{2}\right)^{4}$ results from integrating over the 6-body phase space.

For the MFV flavor breaking case there are several subtleties when calculating the decay width. For instance, the Levi-Civita tensor contractions lead to vanishing operators for some of the color and Lorentz contractions. Another subtlety is that the tree decay may be strongly CKM suppressed so that the leading decay amplitude is the 1-loop one, see Fig. A.1. The decay width can thus be estimated as

$$
\Gamma\left(\chi \rightarrow u_{\mathrm{I}} u_{\mathrm{J}} d_{\mathrm{B}} d_{\mathrm{E}} d_{\mathrm{C}}^{c *} d_{\mathrm{F}}^{c *}\right) \simeq \max \left\{\begin{array}{l}
\frac{\left(\mathcal{C}_{(\mathrm{MFV} V}^{\mathrm{IBCEF}}\right)^{2}}{8 \pi}\left(\frac{1}{16 \pi^{2}}\right)^{4}\left(\frac{m_{\chi}}{\Lambda}\right)^{12} m_{\chi}, \\
\left(\frac{1}{16 \pi^{2}}\left[V_{\mathrm{CKM}}\right]_{\mathrm{IX}}\left[V_{\mathrm{CKM}}\right]_{\mathrm{YE}}\right)^{2} \frac{\left(\mathcal{C}_{(\mathrm{MFV})}^{\mathrm{YIBC}}\right)^{2}}{8 \pi}\left(\frac{1}{16 \pi^{2}}\right)^{4}\left(\frac{m_{\chi}}{\Lambda}\right)^{12} m_{\chi} .
\end{array}\right.
$$

where the first (second) line gives the NDA estimates for the tree level (1-loop) dominated decay width. The $W$ emitted from the left-handed quark lines coming from the effective decay vertex gives the additional CKM factors in the second line.

An example where the leading decay amplitude is generated at 1-loop is the $B=2$ 
ADM with $m_{\chi}=3.3 \mathrm{GeV}$, discussed in Sec. 2.3. Decays into final states with one charm, bottom or top quark are kinematically forbidden. For instance, the lightest $B=2$ final states with one valence charm quark are $\Lambda_{c}^{+}+\Sigma^{-}$and $n+\Sigma_{c}^{0}$. The first has the valence quark content $\sim u d c+d d s$ and the rest mass $m_{\Lambda_{c}^{+}}+m_{\Sigma^{-}}=3.48 \mathrm{GeV}$, while the valence quark content of the second is $\sim u d d+d d c$ and its rest mass $m_{n}+m_{\Sigma_{c}^{0}}=3.4 \mathrm{GeV}$. In contrast, the decays to $\Xi^{0}(\sim u d s)$ or $\Lambda^{0}(\sim u d s)$ baryons are allowed. Eq. (A.29) gives

$$
\begin{aligned}
& \Gamma_{\mathrm{TREE}}^{\mathrm{MFV}}\left(\chi \rightarrow \Lambda^{0} \Lambda^{0}\right) \simeq\left(y_{s} V_{u b}^{2}\right)^{2} \frac{1}{8 \pi}\left(\frac{1}{16 \pi^{2}}\right)^{4}\left(\frac{m_{\chi}}{\Lambda}\right)^{12} m_{\chi}, \\
& \Gamma_{\mathrm{LOOP}}^{\mathrm{MFV}}\left(\chi \rightarrow \Lambda^{0} \Lambda^{0}\right) \simeq\left(\frac{y_{b} V_{u b} V_{t s}}{16 \pi^{2}}\right)^{2} \frac{1}{8 \pi}\left(\frac{1}{16 \pi^{2}}\right)^{4}\left(\frac{m_{\chi}}{\Lambda}\right)^{12} m_{\chi},
\end{aligned}
$$

with the same estimate, within our precision, for the $\chi \rightarrow \Xi^{0}, \Xi^{0}$ or $\chi \rightarrow \Lambda^{0}, \Lambda^{0}$ decays. Note that in the 1-loop amplitude the partonic transition at the decay vertex, $\chi \rightarrow$ $u d b+t d s$, carries no CKM suppression. Furthermore, the $y_{s}$ Yukawa insertion in the tree level amplitude is replaced by $y_{b}$. The $b$ and $t$ quark lines then convert to $u$ and $s$ quark lines via $W$ exchange, as shown in Fig. A.1. The smaller CKM and Yukawa suppressions compensate the loop factor so that the 1-loop amplitude dominates, with the NDA estimate $\Gamma_{\mathrm{LOOP}}^{\mathrm{MFV}} / \Gamma_{\mathrm{TREE}}^{\mathrm{MFV}} \sim \mathcal{O}(10)$.

This procedure can be repeated for different DM masses, arriving at the dominant decay modes as a function of $m_{\chi}$. The results are listed in Table A.1, where we give the kinematical thresholds (1st column) for a number of decay channels (4th column), along with the corresponding partonic transitions (3rd column) and the decay vertex transitions (2nd column). The latter two differ for the loop processes, cf. Fig. A.1. The total decay width for given $m_{\chi}$ is then the sum of partial decay widths, $\Gamma_{i}$, (5th column) for the decay channels that are kinematically allowed. For convenience we also give the decay times, $\tau_{i}$, (6th column) that correspond to individual partial decay widths. Note that in the calculation of the partial decay widths we neglect the phase space 
suppression, while the quoted $\Gamma_{i}$ in Table A.1 are obtained from the NDA estimates (A.29) with $m_{\chi}$ at the kinematical threshold, and setting $\Lambda=1 \mathrm{TeV}$.

In the case of FN flavor breaking the leading tree level and loop induced decay widths for $B=2, m_{\chi}=3.3 \mathrm{GeV}$ DM have the NDA estimates of

$$
\begin{aligned}
\Gamma_{\mathrm{TREE}}^{\mathrm{FN}}\left(\chi \rightarrow \Lambda^{0} \Lambda^{0}\right) & \simeq\left(\lambda^{2}\right)^{2} \frac{1}{8 \pi}\left(\frac{1}{16 \pi^{2}}\right)^{4}\left(\frac{m_{\chi}}{\Lambda}\right)^{12} m_{\chi}, \\
\Gamma_{\mathrm{LOOP}}^{\mathrm{FN}}\left(\chi \rightarrow \Lambda^{0} \Lambda^{0}\right) & \simeq\left(\frac{\lambda^{4}}{16 \pi^{2}}\right)^{2} \frac{1}{8 \pi}\left(\frac{1}{16 \pi^{2}}\right)^{4}\left(\frac{m_{\chi}}{\Lambda}\right)^{12} m_{\chi} .
\end{aligned}
$$

In this case the tree level decay dominates over the loop induced decay by four orders of magnitude. The dominance of the tree level decay amplitude over the 1-loop decay amplitude holds also, if the DM mass is varied. This can be traced to the following difference between the MFV and FN ansätze. In the MFV case the Levi-Civita tensors enforce that two quark flavors in the effective decay vertex need to be from the third generation. This can be changed either by using the $V_{\mathrm{CKM}}$ misalignment or through a loop transition. In FN flavor structure ansatz, on the other hand, the flavor indices need not be antisymmetric.

\section{A.IV Loop functions in neutral meson mixing}

Here we list the analytical form of the loop functions $F(x), F^{F}\left(x_{1}, x_{2}\right), G\left(x_{1}, x_{2}\right), G^{F}\left(x_{1}, x_{2},{ }_{x} 3\right)$ and $H(x), H^{F}\left(x_{1}, x_{2}\right)$ that appear in the 1-loop expressions for the Wilson coefficients in the neutral meson mixing, Sec. 2.6.1. The mediator loop functions with mass degenerate quarks in the loop are given by

$$
F(x)=x H(x), \quad H(x)=\frac{1}{(1-x)^{3}}\left[1-x^{2}+2 x \log (x)\right]
$$


while for two different quarks running in the loop they are

$$
\begin{aligned}
& F^{F}\left(x_{i}, x_{j}\right)=\frac{x_{i} x_{j}}{\left(1-x_{i}\right)\left(1-x_{j}\right)}+\left[\frac{x_{i} x_{j} \log \left(x_{i}\right)}{\left(1-x_{i}\right)^{2}\left(x_{i}-x_{j}\right)}+x_{i} \leftrightarrow x_{j}\right], \\
& H^{F}\left(x_{i}, x_{j}\right)=\frac{1}{\left(1-x_{i}\right)\left(1-x_{j}\right)}+\left[\frac{x_{i}^{2} \log \left(x_{i}\right)}{\left(1-x_{i}\right)^{2}\left(x_{i}-x_{j}\right)}+x_{i} \leftrightarrow x_{j}\right] .
\end{aligned}
$$

The loop functions for the mediator- $W$ loops are

$$
\begin{gathered}
G\left(x_{w}, x\right)=\frac{x}{x_{w}}\left[\frac{x_{w}+x}{(x-1)\left(x-x_{w}\right)}-\frac{x\left(2 x x_{w}-x_{w}^{2}-2 x_{w}+x^{2}\right) \log (x)}{(x-1)^{2}\left(x-x_{w}\right)^{2}}\right. \\
\left.\quad+\frac{2 x x_{w} \log \left(x_{w}\right)}{\left(x-x_{w}\right)^{2}\left(x_{w}-1\right)}\right] \\
G^{F}\left(x_{w}, x_{i}, x_{j}\right)=\frac{x_{i} x_{j}}{x_{w}}\left[\left\{\frac{\left(x_{i}+x_{w}\right) \log \left(x_{i}\right)}{\left(1-x_{i}\right)\left(x_{i}-x_{j}\right)\left(x_{w}-x_{i}\right)}+x_{i} \leftrightarrow x_{j}\right\}\right. \\
\left.-\frac{2 x_{w} \log \left(x_{w}\right)}{\left(1-x_{w}\right)\left(x_{w}-x_{i}\right)\left(x_{w}-x_{j}\right)}\right] .
\end{gathered}
$$




\begin{tabular}{ccccccc}
\hline \hline $\mathrm{T} / \mathrm{h}[\mathrm{GeV}]$ & Decay vertex & Partonic & Final State & $\Gamma_{i}[\mathrm{GeV}]$ & $\tau_{i}[\mathrm{~s}]$ & Process \\
\hline 2.06 & $\chi \rightarrow$ cudbsd & $\chi \rightarrow$ udduds & $n+\Lambda^{0}$ & $1.34 \times 10^{-60}$ & $4.91 \times 10^{35}$ & Loop \\
2.23 & $\chi \rightarrow$ uusdsd & $\chi \rightarrow$ uds uds & $\Lambda^{0}+\Lambda^{0}$ & $3.74 \times 10^{-55}$ & $1.76 \times 10^{30}$ & Tree \\
2.43 & $\chi \rightarrow$ cusbsd & $\chi \rightarrow$ uds uss & $\Lambda^{0}+\Xi^{0}$ & $4.25 \times 10^{-57}$ & $1.55 \times 10^{32}$ & Loop \\
3.48 & $\chi \rightarrow$ ccdssd & $\chi \rightarrow$ udcdds & $\Lambda_{c}^{+}+\Sigma^{-}$ & $1.23 \times 10^{-55}$ & $5.33 \times 10^{30}$ & Loop \\
3.61 & $\chi \rightarrow$ ucsdsd & $\chi \rightarrow u d c d s s$ & $\Lambda_{c}^{+}+\Xi^{-}$ & $1.87 \times 10^{-50}$ & $3.52 \times 10^{25}$ & Tree \\
3.81 & $\chi \rightarrow$ ccsdsd & $\chi \rightarrow u d s s s c$ & $\Lambda^{0}+\Omega_{c}^{0}$ & $1.42 \times 10^{-52}$ & $4.62 \times 10^{27}$ & Loop \\
3.79 & $\chi \rightarrow$ ccsdsd & $\chi \rightarrow u s c d s s$ & $\Xi_{c}^{+}+\Xi^{-}$ & $1.33 \times 10^{-52}$ & $4.96 \times 10^{27}$ & Loop \\
4.63 & $\chi \rightarrow$ ccdbsd & $\chi \rightarrow d c c d d s$ & $\Xi_{c c}^{+}+\Sigma^{-}$ & $1.01 \times 10^{-52}$ & $6.51 \times 10^{27}$ & Loop \\
4.93 & $\chi \rightarrow$ ccsdsd & $\chi \rightarrow d d c d s c$ & $\Sigma_{c}^{0}+\Xi_{c}^{0}$ & $1.04 \times 10^{-46}$ & $6.33 \times 10^{21}$ & Tree \\
5.17 & $\chi \rightarrow$ ccsbsd & $\chi \rightarrow d s c s s c$ & $\Xi_{c}^{0}+\Omega_{c}^{0}$ & $4.14 \times 10^{-52}$ & $1.59 \times 10^{27}$ & Loop \\
6.56 & $\chi \rightarrow$ cudsbd & $\chi \rightarrow u d d u d b$ & $n+\Lambda_{b}^{0}$ & $7.25 \times 10^{-54}$ & $9.08 \times 10^{28}$ & Loop \\
6.73 & $\chi \rightarrow$ uudbsd & $\chi \rightarrow u d d u s b$ & $n+\Xi_{b}^{0}$ & $1.87 \times 10^{-45}$ & $3.53 \times 10^{20}$ & Tree \\
6.94 & $\chi \rightarrow$ uusbsd & $\chi \rightarrow u s s u d b$ & $\Xi^{0}+\Lambda_{b}^{0}$ & $5.18 \times 10^{-44}$ & $1.27 \times 10^{19}$ & Tree \\
7.10 & $\chi \rightarrow$ cusdbs & $\chi \rightarrow u s s u s b$ & $\Xi^{0}+\Xi_{b}^{0}$ & $3.49 \times 10^{-52}$ & $1.89 \times 10^{27}$ & Loop \\
8.07 & $\chi \rightarrow$ ucbdbd & $\chi \rightarrow u d b d d c$ & $\Lambda_{b}^{0}+\Sigma_{c}^{0}$ & $2.16 \times 10^{-51}$ & $3.05 \times 10^{26}$ & Loop \\
8.09 & $\chi \rightarrow$ ucbdsd & $\chi \rightarrow u d b d s c$ & $\Lambda_{b}^{0}+\Xi_{c}^{0}$ & $2.00 \times 10^{-42}$ & $3.30 \times 10^{17}$ & Tree \\
8.31 & $\chi \rightarrow$ ucbssd & $\chi \rightarrow u d b s s c$ & $\Lambda_{b}^{0}+\Omega_{c}^{0}$ & $5.33 \times 10^{-41}$ & $1.24 \times 10^{16}$ & Tree \\
8.48 & $\chi \rightarrow$ ucbsbs & $\chi \rightarrow u s b s s c$ & $\Xi_{b}^{0}+\Omega_{c}^{0}$ & $7.66 \times 10^{-50}$ & $8.59 \times 10^{24}$ & Loop \\
11.24 & $\chi \rightarrow$ uubdbd & $\chi \rightarrow u d b u d b$ & $\Lambda_{b}^{0}+\Lambda_{b}^{0}$ & $2.24 \times 10^{-42}$ & $2.93 \times 10^{17}$ & Tree \\
11.41 & $\chi \rightarrow$ uubbsd & $\chi \rightarrow u d b u s b$ & $\Lambda_{b}^{0}+\Xi_{b}^{0}$ & $9.86 \times 10^{-38}$ & $6.68 \times 10^{12}$ & Tree \\
11.58 & $\chi \rightarrow$ uubsbs & $\chi \rightarrow u s b u s b$ & $\Xi_{b}^{0}+\Xi_{b}^{0}$ & $2.94 \times 10^{-42}$ & $2.24 \times 10^{17}$ & Tree \\
\hline \hline
\end{tabular}

Table A.1: Partial decay widths, $\Gamma_{i}$, and related decay times, $\tau_{i}=1 / \Gamma_{i}$, for representative decay channels above kinematical thresholds (1st column) assuming the MFV flavor breaking ansatz. The EFT scale is set to $\Lambda=1 \mathrm{TeV}$. The last column denotes whether the dominant amplitude is tree level or 1-loop, while the 2nd and the 3rd columns give the decay vertex transition and the partonic transition after the potential $W$ exchange, respectively. 


\section{B - Appendix to Chapter 3}

\section{B.I Minimal flavour violation with gauged flavor sym- metries}

In this appendix we verify numerically that the Wilson coefficients in the weak Hamiltonian for $B_{d}$ and $B_{s}$ mixing,

$$
\mathcal{H}_{\text {eff }}^{\Delta B=2}=\sum_{i=1}^{5} C_{i}^{b q} Q_{i}^{b q}+\sum_{i=1}^{5} \tilde{C}_{i}^{b q} \tilde{Q}_{i}^{b q},
$$

generated from exchanges of flavored gauge bosons, are of the MFV type. A tree level exchange of FGB generates contributions to $B_{d}$ mixing through operators

$$
Q_{1}^{b d}=\bar{d}_{L}^{\alpha} \gamma_{\mu} b_{L}^{\alpha} \bar{d}_{L}^{\beta} \gamma_{\mu} b_{L}^{\beta}, \quad Q_{3}^{b d}=\bar{d}_{R}^{\alpha} b_{L}^{\beta} \bar{d}_{R}^{\beta} b_{L}^{\alpha}
$$

and $\tilde{Q}_{1}^{b d}, \tilde{Q}_{3}^{b d}$, that follow from $Q_{1}^{b d}, Q_{3}^{b d}$ with $P_{L} \leftrightarrow P_{R}$ exchange (the remaining operators can be found in, e.g., Ref. [82]). If the lightest FGB has predominantly left-handed couplings, then $C_{1}^{b d}$ Wilson coefficient is the largest one, while if the lightest FGB is coupling predominantly to the right-handed quarks, the $\tilde{C}_{1}^{b d}$ dominates. For comparable left-handed and right-handed couplings all four Wilson coefficients, $C_{1,3}^{b d}, \tilde{C}_{1,3}^{b d}$, are important. Analogous discussion applies to the case of FGB contributions to $B_{s}$ mixing which are obtained with a trivial $d \rightarrow s$ exchange. 

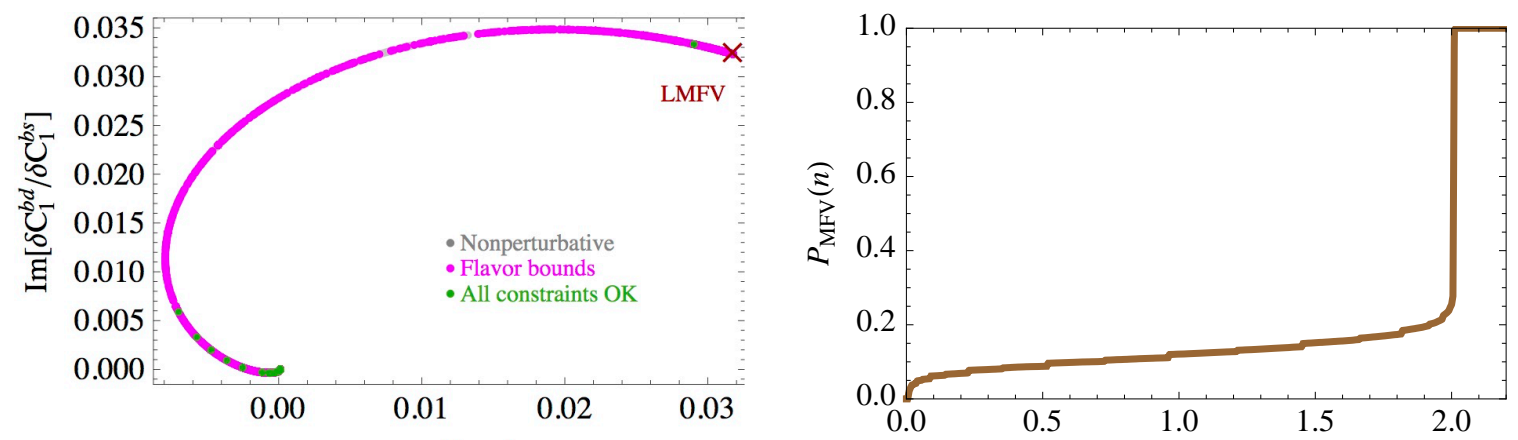

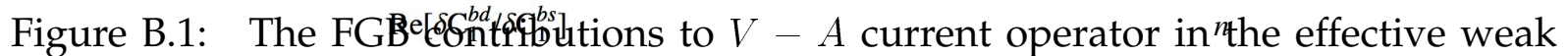
Hamiltonian. Left panel shows the values of the complex ratio $\delta C_{1}^{b d} / \delta C_{1}^{b s}$ for our scan points, with green points satisfying all constraints, magenta points excluded by flavor constraints and grey points by perturbativity considerations. The point $\delta C_{1}^{b d} / \delta C_{1}^{b s}=\left(V_{t d}^{*} / V_{t s}^{*}\right)^{2}$, obtained if the MFV operator with the smallest number of Yukawa insertions dominates, is denoted by a cross. The right panel shows the cumulative function $P_{M F V}(n)$, see Eq. (B.4).

As discussed in Section 3.3 the contributions from the gauged flavor model can be expanded in terms of the SM Yukawas. The contributions due to FGB exchanges can thus be written as

$$
\delta C_{1}^{b d}=c_{1}\left(y_{u} y_{u}^{\dagger}\right)_{13}^{2}+c_{2}\left(y_{u} y_{u}^{\dagger}\right)_{13}\left(y_{d} y_{d}^{\dagger} y_{u} y_{u}^{\dagger}\right)_{13}+\cdots=c_{1}\left(V_{t d}^{*} V_{t b}\right)^{2}+c_{2} y_{d}^{2}\left(V_{t d}^{*} V_{t b}\right)^{2}+\cdots
$$

where $\left(y_{d}\right)_{i j}=\operatorname{diag}\left(y_{d}, y_{s}, y_{b}\right)$, and we have set $y_{t}=1$ in the second equality. In (B.3) we kept only the two terms relevant for the discussion below. The same expansion applies for $\delta C_{1}^{b s}$ with $d \rightarrow s$ replacement in (B.3).

In Fig. B.1 (left) we show the ratio $\delta C_{1}^{b d} / \delta C_{1}^{b s}$, i.e. the NP contribution to the $V-A$ quark current operator due to tree level FGB exchanges. Note that the ratio $\delta C_{1}^{b d} / \delta C_{1}^{b s}$ can be complex. If the $c_{1}$ term dominates then $\delta C_{1}^{b d} / \delta C_{1}^{b s} \simeq\left(V_{t d}^{*} / V_{t s}^{*}\right)^{2}$. This is denoted by a cross in Fig. B.1 (left). The addition of the operators with extra insertions of $y_{d} y_{d}^{\dagger}$ lead to $\delta C_{1}^{b d} / \delta C_{1}^{b s}$ not beign equal to $\left(V_{t d}^{*} / V_{t s}^{*}\right)^{2}$. We have verified that the curve for $\delta C_{1}^{b d} / \delta C_{1}^{b s}$ shown in Fig. B.1 (left) can be fit with the form of $\delta C_{1}^{b d, b s}$ in (B.3), taking $c_{1}$ real and $c_{2}$ complex, where $c_{1}$ varies from being $\mathcal{O}(1)$ to being vanishingly small. The points in our scan can be grouped into two sets. For the first set of points both $c_{1}$ 
and $c_{2}$ terms are sizeable. For the second set of points the $c_{1}$ term is negligible and $c_{2}$ dominates. This is shown in Fig. B.1 (right) where we plot the cumulative distribution function

$$
P_{\mathrm{MFV}}(n)=\frac{N\left(\left|\delta C_{1}^{b d} / \delta C_{1}^{b s}\right| \geq\left(m_{d} / m_{s}\right)^{n}\left|V_{t d}^{*} / V_{t s}^{*}\right|^{2}\right)}{N_{\text {total }}}
$$

The function $P_{\mathrm{MFV}}(n)$ can be interpreted as the fraction of points that have the ratio $\left|\delta C_{1}^{b d} / \delta C_{1}^{b s}\right|$ effectively dominated by operators with up to $y_{d}^{n}$ insertions. That is, the points dominated by the $c_{1}$ term contribute to $P_{\mathrm{MFV}}(0)$ (and to $P_{\mathrm{MFV}}(n)$ with $n \geq 0$ ), while the points dominated by the $c_{2}$ term ontribute to $P_{\mathrm{MFV}}(2)$ (and to $P_{\mathrm{MFV}}(n)$ with $n \geq 2$ ). The points with both $c_{1}$ and $c_{2}$ start contributing to $P_{\mathrm{MFV}}(n)$ for $n$ somewhere between 0 and 2, depending on the relative sizes of $c_{1}$ and $c_{2}$. Fig. B.1 (right) shows that a subleading (but nonzero) set of points is dominated by the $c_{1}$ term, about $10 \%$ points have both $c_{1}$ and $c_{2}$ terms sizeable, while about $80 \%$ of points are dominated by the $c_{2}$ term.

Similar analysis can be made for $V+A$ operators, $\tilde{Q}_{1}^{b d}$ and $\tilde{Q}_{1}^{b s}$. One can expand the FGB contributions to their respective Wilson coefficients in terms of the SM Yukawas

$$
\begin{aligned}
\delta \tilde{C}_{1}^{b d} & =\tilde{c}_{1}\left(y_{d}^{\dagger} y_{u} y_{u}^{\dagger} y_{b}\right)_{13}^{2}+\tilde{c}_{2}\left(y_{d}^{\dagger} y_{u} y_{u}^{\dagger} y_{b}\right)_{13}\left(y_{d}^{\dagger} y_{d} y_{d}^{\dagger} y_{u} y_{u}^{\dagger} y_{b}\right)_{13}+\cdots \\
& =\tilde{c}_{1} y_{d}^{2} y_{b}^{2}\left(V_{t d}^{*} V_{t b}\right)^{2}+\tilde{c}_{2} y_{d}^{4} y_{b}^{2}\left(V_{t d}^{*} V_{t b}\right)^{2}+\cdots
\end{aligned}
$$

and similarly for $\delta \tilde{C}_{1}^{b s}$ with $d \rightarrow s$ replacement. We show in Fig. B.2 (left) the ratio $\tilde{C}_{1}^{b d} / \tilde{C}_{1}^{b s}$ for our scan. If the $\tilde{c}_{1}$ term dominates, then $\tilde{C}_{1}^{b d} / \tilde{C}_{1}^{b s}=\left(m_{d} / m_{s}\right)^{2}\left(V_{t d}^{*} / V_{t s}^{*}\right)^{2}$, which is denoted by the cross in Fig. B.2 (left). The points for which also the $\tilde{c}_{2}$ operator (and other operators denoted by ellipses above) is important then lie away from the $\tilde{C}_{1}^{b d} / \tilde{C}_{1}^{b s}=\left(m_{d} / m_{s}\right)^{2}\left(V_{t d}^{*} / V_{t s}^{*}\right)^{2}$ region. We also define a cumulative function

$$
\tilde{P}_{\mathrm{MFV}}(n)=\frac{N\left(\left|\delta \tilde{C}_{1}^{b d} / \delta \tilde{C}_{1}^{b s}\right| \geq\left(m_{d} / m_{s}\right)^{n+2}\left|V_{t d}^{*} / V_{t s}^{*}\right|^{2}\right)}{N_{\text {total }}} .
$$



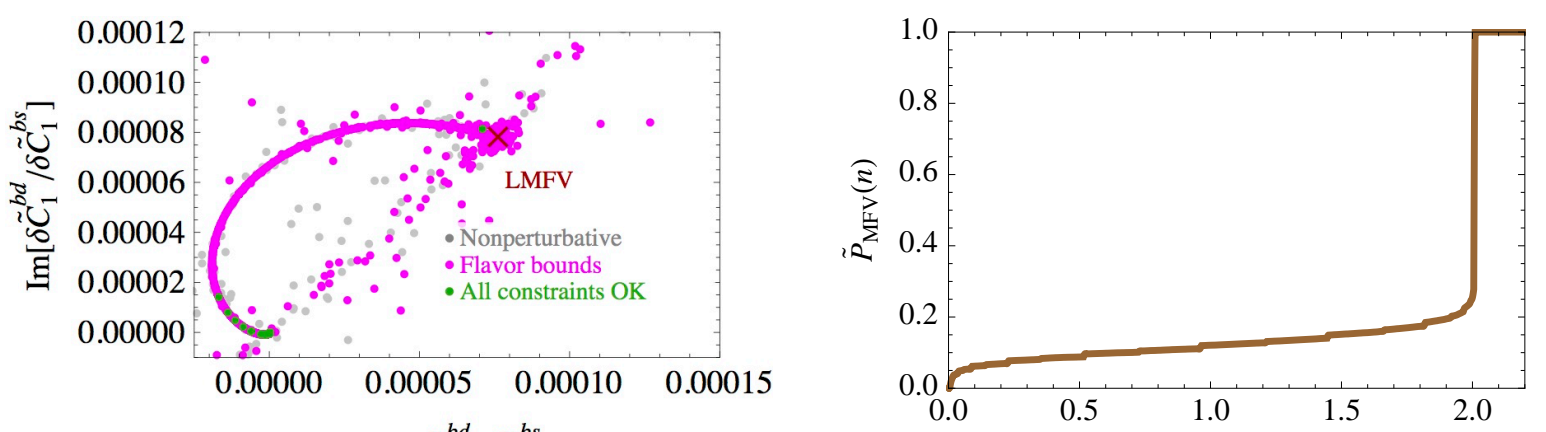

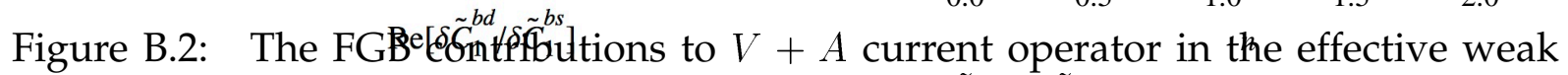
Hamiltonian. Left panel shows the complex ratio $\delta \tilde{C}_{1}^{b d} / \delta \tilde{C}_{1}^{b s}$ for our scan points with the same color coding as in Fig. B.1. The point $\delta \tilde{C}_{1}^{b d} / \delta \tilde{C}_{1}^{b s}=\left(m_{d} / m_{s}\right)^{2}\left(V_{t d}^{*} / V_{t s}^{*}\right)^{2}$, obtained if the MFV operator with the smallest number of Yukawa insertions dominates, is denoted by a cross. The right panel shows the cumulative function $\tilde{P}_{\mathrm{MFV}}(n)$, see Eq. (B.6).

The values for $\tilde{P}_{\mathrm{MFV}}(n)$ are shown in Fig. B.2 (right). We see that also in this case the points cluster into two groups, with vanishing $\tilde{c}_{1}$ term or with both $\tilde{c}_{1}$ and $\tilde{c}_{2}$ relevant.

The above analysis demonstrates that the FGB contributions to the Wilson coefficients in the effective weak hamiltonian can be expanded in terms of the SM Yukawas which is a hallmark of (general) MFV. In particular, the expansion in terms of $m_{d, s} / m_{b}$ and off-diagonal CKM elements can be still performed and is not ruined by the large ratios of scales present in the problem such as the very disparate FGB masses.

\section{B.II Thermal relic computation}

In this appendix we describe the calculation of relic density that was used in the scans in the main part of the paper. Several approximations to the coupled Boltzmann equations were necessary in order to reduce the evaluation time per benchmark and thus allow adequate coverage of the parameter space. We find the approximate solutions to be in agreement with the full solutions at the $\mathcal{O}(30 \%)$ level. The full numerical solution of the Boltzmann equations was obtained with MadDM [105] using a UF0 model file [310], which was generated with the FeynRules package [106]. 
Denoting the DM multiplet by $\varphi$, where $\varphi$ is either a Dirac fermion or a complex scalar, the most general set of coupled Boltzmann equations reads [103]

$$
\begin{aligned}
\frac{d n_{\varphi_{i}}}{d t}+3 H n_{\varphi_{i}}= & -\sum_{j}\left\langle\sigma\left(\varphi_{i} \varphi_{j} \leftrightarrow X X\right) v_{\mathrm{lab}}\right\rangle\left(n_{\varphi_{i}} n_{\varphi_{j}}-n_{\varphi_{i}}^{\mathrm{EQ}} n_{\varphi_{j}}^{\mathrm{EQ}}\right) \\
& -\sum_{j \neq i}\left\langle\sigma\left(\varphi_{i} X \leftrightarrow \varphi_{j} X\right) v_{\mathrm{lab}}\right\rangle\left(n_{\varphi_{i}}-\frac{n_{\varphi_{i}}^{\mathrm{EQ}}}{n_{\varphi_{j}}^{\mathrm{EQ}}} n_{\varphi_{j}}\right) n_{X}^{\mathrm{EQ}} \\
& -\sum_{j \neq i}\left\langle\sigma\left(\varphi_{i} \varphi_{j} \leftrightarrow \varphi_{k} \varphi_{\ell}\right) v_{\mathrm{lab}}\right\rangle\left(n_{\varphi_{i}} n_{\varphi_{j}}-\frac{n_{\varphi_{i}}^{\mathrm{EQ}} n_{\varphi_{j}}^{\mathrm{EQ}}}{n_{\varphi_{k}}^{\mathrm{EQ}} \varphi_{\ell}^{\mathrm{EQ}}} n_{\varphi_{k}} n_{\varphi_{\ell}}\right) \\
& \pm \sum_{j \neq i}\left[\left\langle\Gamma\left(\varphi_{j, i} \rightarrow \varphi_{i, j} X\right)\right\rangle n_{\varphi_{j, i}}+\left\langle\sigma\left(\varphi_{j, i} X \rightarrow \varphi_{i, j}\right)\right\rangle n_{\varphi_{j, i}} n_{X}^{\mathrm{EQ}}\right],
\end{aligned}
$$

where $X$ denotes a generic SM state. For large mass splittings between the $\varphi$ components it is sufficient to consider the lightest $\varphi_{i}$ state in Eq. (B.7). The contributions to the DM relic density from the heavy $\varphi$ components are exponentially suppressed by corresponding Boltzmann factors and can be neglected within our precision. In contrast, when the mass splittings are small the full set of coupled Boltzmann equations in Eq. (B.7) needs to be considered. Nevertheless, even in this case several approximations are possible for our model, as we explain below.

First of all, the coannihilation of different $\varphi_{i}$ components into SM particles, $\varphi_{i} \varphi_{j} \rightarrow$ $X X(i \neq j)$, can be safely neglected in our model. In benchmarks that survive the experimental constraints the off-diagonal couplings of the lightest FGB to $\varphi$ are much smaller than the diagonal ones, see Fig. B.3. Secondly, in the calculation of DM relic density we also neglect the flavor-changing DM scattering off the thermal background, $\varphi_{i} X \rightarrow \varphi_{j} X$. The $\varphi_{i} X \rightarrow \varphi_{j} X$ scattering can be important if $\left\langle\sigma\left(\varphi_{i} X \rightarrow \varphi_{j} X\right) v_{\text {lab }}\right\rangle /\left\langle\sigma\left(\varphi_{i} \varphi_{j} \rightarrow X X\right) v_{\text {lab }}\right\rangle$ $n_{\varphi_{j}} / n_{X}^{\mathrm{EQ}} \sim 10^{-9}$. In this case the off-diagonal couplings of $\mathcal{O}\left(10^{-4}\right)$ relative to the diagonal ones are in principle large enough to have $\mathcal{O}(1)$ effects on the relic density, and neglecting $\varphi_{i} X \rightarrow \varphi_{j} X$ may not be justified. Therefore, for the benchmarks with $\left(\hat{g}_{\chi}^{24}\right)_{23} /\left(\hat{g}_{\chi}^{24}\right)_{33}>3 \times 10^{-4}$ and small mass splittings among $\varphi$, we explicitly verified us- 


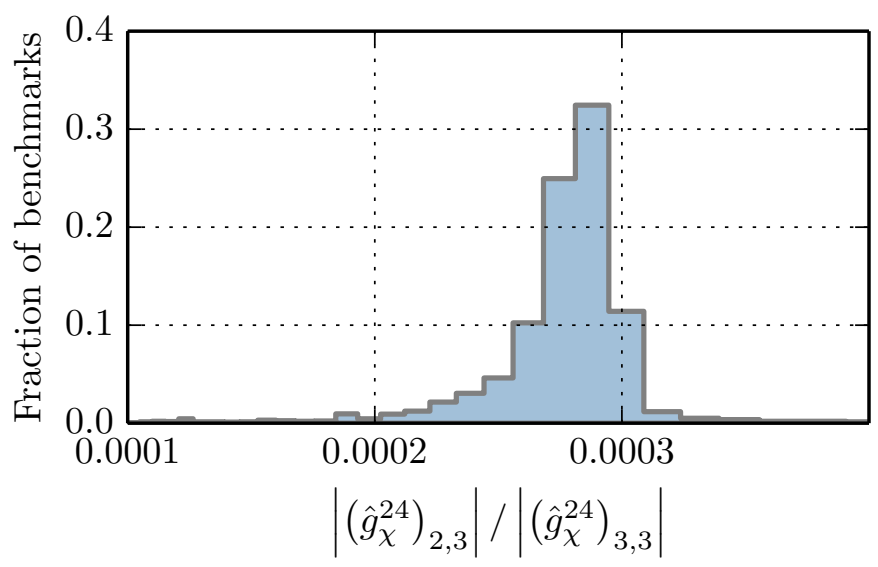

Figure B.3: The fraction of benchmarks as a function of the off-diagonal couplings of the heaviest and next-to-heaviest DM components to the lightest FGB $\left(A^{24}\right)$ normalized by the diagonal coupling of the heaviest component.

ing MadDM that neglecting $\varphi_{i} X \rightarrow \varphi_{j} X$ scattering leads to a change in DM relic density smaller than $\mathcal{O}(30 \%)$.

Finally, we are able to neglect the pure DM scattering process in the third line of Eq. (B.7) since $\left\langle\sigma\left(\varphi_{i} \varphi_{j} \leftrightarrow \varphi_{k} \varphi_{\ell}\right) v_{\text {lab }}\right\rangle \ll\left\langle\sigma\left(\varphi_{i} \varphi_{j} \leftrightarrow X X\right) v_{\text {lab }}\right\rangle$ in our model. The largest contribution to this process is from diagonal couplings between the FGB and DM. This process can couple the evolution of the DM species if $\left\langle\sigma\left(\varphi_{i} \varphi_{j} \leftrightarrow \varphi_{k} \varphi_{\ell}\right) v_{\mathrm{lab}}\right\rangle \sim$ $\left\langle\sigma\left(\varphi_{i} \varphi_{j} \leftrightarrow X X\right) v_{\text {lab }}\right\rangle$. The diagonal FGB couplings to the quarks and to the DM of the same generation are approximately equal. By accounting for the color factors and the multiplicity of channels when annihilating into SM fields one concludes that the pure DM scattering is indeed subleading.

Therefore, for almost mass degenerate $\varphi_{i}$ it is sufficient to consider a set of uncoupled Boltzmann equations

$$
\frac{d n_{\varphi_{i}}}{d t}+3 H n_{\varphi_{i}}=-\sum_{j}\left\langle\sigma\left(\varphi_{i} \varphi_{i} \leftrightarrow X X\right) v_{\mathrm{lab}}\right\rangle\left(n_{\varphi_{i}}^{2}-n_{\varphi_{i}}^{\mathrm{EQ} 2}\right)
$$

The DM relic abundance is in this case the sum of relic abundances for each of the 
three components obtained from the above set of equations (the heavy $\varphi$ components, in our case $\varphi_{2}$ and $\varphi_{3}$, decay after their respective freeze-outs and contribute to the $\varphi_{1}$ DM relic abundance). In contrast, for large mass splittings the heavy $\varphi$ components are irrelevant for the calculation of the DM relic abundance. This is then obtained from Eq. (B.8) by considering only the lightest DM state, in our case $\varphi_{1}$.

We calculate the DM relic abundance from Eq. (B.8) using the freeze-out approximation [104], which gives

$$
\Omega h^{2}=\frac{1.07 \times 10^{9} \mathrm{GeV}^{-1}}{J\left(x_{f}\right) \sqrt{g_{*}} M_{P l}},
$$

where $M_{P l}=1.22 \times 10^{19} \mathrm{GeV}, g_{*}$ is the total number of effectively relativistic degrees of freedom at the time of the freeze-out, and

$$
J\left(x_{f}\right)=\int_{x_{f}}^{\infty} d x \frac{\left\langle\sigma v_{\mathrm{lab}}\right\rangle_{\mathrm{th}}}{x^{2}} .
$$

The freeze-out temperature $\left(x_{f}=m_{\varphi_{1}} / T_{f}\right)$ is obtained by solving

$$
x_{f}=\ln \frac{0.038 g_{\mathrm{eff}} M_{P l} m_{\varphi_{1}}\left\langle\sigma v_{\mathrm{lab}}\right\rangle_{\mathrm{th}}}{\sqrt{g_{*} x_{f}}}
$$

where the thermally-averaged cross section is

$$
\left\langle\sigma v_{\mathrm{lab}}\right\rangle_{\mathrm{th}}=\frac{2 x^{\frac{3}{2}}}{\sqrt{\pi}} \int_{0}^{\infty} \sigma_{\mathrm{eff}} v_{\mathrm{lab}} \sqrt{\epsilon} e^{-x \epsilon} d \epsilon
$$

with $v_{\text {lab }}=2 \sqrt{\epsilon(1+\epsilon)} /(1+2 \epsilon)$ and $\epsilon=s /\left(2 m_{\varphi_{1}}\right)^{2}-1$. The freeze-out approximation is accurate to a few percent with respect to the full numerical solution of the Boltzmann equation [103].

The fermionic flavored DM annihilates through the s-channel exchange of FGBs. In this case, the integration over $x$ can be performed analytically and the double integral in Eq. (B.10) reduces to a single one that can be efficiently evaluated numerically. In 
particular,

$$
J\left(x_{f}\right)=\int_{0}^{\infty} 2 \sigma v_{\mathrm{lab}} \operatorname{Erfc}\left(\sqrt{x_{f}}\right) d \epsilon .
$$

We evaluate the above integral numerically in the parameter scan.

In the annihilation cross section of the fermionic flavored DM we keep the dominant contribution - the $s$-channel exchange of the lightest FGB, $A^{24}$. The annihilation cross section for $\chi_{i} \bar{\chi}_{i} \rightarrow \bar{u}_{j} u_{j}$ (and similarly for $\chi_{i} \bar{\chi}_{i} \rightarrow \bar{d}_{j} d_{j}$ ) is given by

$$
\begin{aligned}
\sigma\left(\chi_{i} \bar{\chi}_{i} \rightarrow \bar{u}_{j} u_{j}\right)= & \frac{\left(\hat{g}_{\chi}^{24}\right)_{i i}^{2}}{4 \pi} \sqrt{\frac{s-4 m_{u_{j}}^{2}}{s-4 m_{\chi_{i}}^{2}}}\left(1+\frac{2 m_{\chi_{i}}^{2}}{s}\right) \times \\
& \times \frac{\left(\hat{\mathcal{G}}_{V}^{u}\right)_{j j, 24}^{2}\left(s+2 m_{u_{j}}^{2}\right)+\left(\hat{\mathcal{G}}_{A}^{u}\right)_{j j, 24}^{2}\left(s-4 m_{u_{j}}^{2}\right)}{\left(s-m_{A^{24}}^{2}\right)^{2}+m_{A^{24}}^{2} \Gamma_{A^{24}}^{2}},
\end{aligned}
$$

where the vector and axial-vector couplings to quarks, $\hat{\mathcal{G}}_{A, V}$, were defined in Eq. (3.25), $\sqrt{s}$ is the center-of-mass energy and $\Gamma_{A^{24}}$ is the total decay width of the lightest FGB. The decay rate for $A^{24} \rightarrow \bar{u}_{j} u_{j}$ assuming $m_{A^{24}}>2 m_{u_{j}}$ is

$$
\Gamma\left(A^{24} \rightarrow \bar{u}_{j} u_{j}\right)=\frac{m_{A}^{24}}{4 \pi} \sqrt{1-\frac{4 m_{u_{j}}^{2}}{m_{A^{24}}^{2}}}\left[\left(\hat{\mathcal{G}}_{V}^{u}\right)_{j j, 24}^{2}\left(1+\frac{2 m_{u_{j}}^{2}}{m_{A^{24}}^{2}}\right)+\left(\hat{\mathcal{G}}_{A}^{u}\right)_{j j, 24}^{2}\left(1-\frac{4 m_{u_{j}}^{2}}{m_{A^{24}}^{2}}\right)\right] .
$$

The rate for $A^{24} \rightarrow \chi_{i} \bar{\chi}_{i}, \bar{d}_{j} d_{j}$ is obtained after trivial replacements for masses and couplings (and dividing by the $N_{c}$ color factor for decays to $\chi_{i} \bar{\chi}_{i}$ ). The total FGB decay rate is obtained after summing over all kinematically allowed decay channels. 


\section{B.III Higgs coupling Feynman rules}

As noted in Sec. 3.4.5, the $h \bar{f} f$ Feynman rules given in Appendix A.1 of Ref. [102] contain a typo. The corrected Feynman rules are given here.

$$
h-\cdots \varkappa_{f}^{\bar{f}}=\frac{i}{\sqrt{2}}\left(C_{L} P_{L}+C_{R} P_{R}\right)
$$

where the couplings $C_{L}$ and $C_{R}$ are:

$$
\begin{aligned}
& h \bar{u}_{i} u_{i} \quad: C_{L}=C_{R}=+\lambda_{u} s_{u_{R} i} c_{u_{L} i} \\
& h \bar{u}_{i}^{\prime} u_{i}^{\prime} \quad: C_{L}=C_{R}=-\lambda_{u} c_{u_{R} i} s_{u_{L} i} \\
& h \bar{u}_{i} u_{i}^{\prime}:\left\{\begin{array}{l}
C_{R}=-\lambda_{u} c_{u_{R} i} c_{u_{L} i} \\
C_{L}=+\lambda_{u} s_{u_{R} i} s_{u_{L} i}
\end{array}\right. \\
& h \bar{u}_{i}^{\prime} u_{i}:\left\{\begin{array}{l}
C_{R}=+\lambda_{u} s_{u_{R} i} s_{u_{L} i} \\
C_{L}=-\lambda_{u} c_{u_{R} i} c_{u_{L} i}
\end{array}\right.
\end{aligned}
$$




\section{C - Appendix to Chapter 5}

\section{C.I Spinor-helicity formalism}

In this paper we extensively use the spinor-helicity formalism, in which the sigma matrices $\sigma_{a \dot{a}}^{\mu}$ solder null momenta to Weyl spinors, that transform under the spinor irreducible representations of the covering group $\mathrm{SL}(2, \mathbb{C})$. For a review see e.g. Ref. [311] or [312].

For a null momentum $k$, the associated Weyl spinors $\lambda_{k}$ are soldered via

$$
k^{\mu}=\frac{1}{2}\left\langle k^{ \pm}\left|\sigma^{\mu}\right| k^{ \pm}\right\rangle, \quad \not k \equiv k_{\mu} \sigma_{a \dot{a}}^{\mu}=\left(\lambda_{k}\right)_{a}\left(\lambda_{k}\right)_{\dot{a}},
$$

in which we have written the dotted and undotted Weyl spinors in the notation

$$
\left\langle k^{-}\left|\equiv\left(\lambda_{k}\right)^{a}, \quad\left\langle k^{+}\left|\equiv\left(\lambda_{k}\right)_{\dot{a}}, \quad\right| k^{+}\right\rangle \equiv\left(\lambda_{k}\right)_{a}, \quad\right| k^{-}\right\rangle \equiv\left(\lambda_{k}\right)^{\dot{a}}
$$

so $\left\langle k^{+}\left|\sigma^{\mu}\right| \ell^{+}\right\rangle=\left(\lambda_{k}\right)_{\dot{a}} \sigma^{\mu \dot{a} a}\left(\lambda_{\ell}\right)_{a},\left\langle k^{-}\left|\sigma^{\mu}\right| \ell^{-}\right\rangle=\left(\lambda_{k}\right)^{a} \sigma_{a \dot{a}}^{\mu}\left(\lambda_{\ell}\right)^{\dot{a}}$. Upper and lower indices are related by the usual epsilon contractions, in particular $\sigma_{\mu}^{\dot{a} a} \equiv \epsilon^{a b} \epsilon^{\dot{a} \dot{b}} \sigma_{\mu_{b} \dot{b}}$. In this notation, the sign superscripts denote the helicities of the in-going and out-going states, corresponding to kets and bras respectively. In particular, note that $\left|k^{ \pm}\right\rangle^{\dagger}=\left\langle k^{ \pm}\right|$by construction, $\left\langle k^{ \pm}\left|\sigma^{\mu}\right| \ell^{\mp}\right\rangle$ do not exist, while $\left\langle k^{-}\left|\sigma^{\mu}\right| \ell^{-}\right\rangle=\left\langle\ell^{+}\left|\sigma^{\mu}\right| k^{+}\right\rangle$. For null momenta 
$k$ and $\ell$ we often write the bilinears

$$
\langle k \ell\rangle \equiv\left\langle k^{-} \mid \ell^{+}\right\rangle=\left(\lambda_{k}\right)^{a}\left(\lambda_{\ell}\right)_{a}, \quad[k \ell] \equiv\left\langle k^{+} \mid \ell^{-}\right\rangle=\left(\lambda_{k}\right)_{\dot{a}}\left(\lambda_{\ell}\right)^{\dot{a}},
$$

so $\langle\ell k\rangle=-\langle k \ell\rangle,[\ell k]=-[k \ell],\langle k \ell\rangle[\ell k]=2 k \cdot \ell$ and $\langle k \ell\rangle^{*}=[\ell k]$.

In this notation, definite helicity polarization vectors associated to $k$ may have form

$$
\varepsilon^{ \pm \mu}(k ; r)= \pm \frac{\left\langle r^{\mp}\left|\sigma^{\mu}\right| k^{\mp}\right\rangle}{\sqrt{2}\left\langle r^{\mp} \mid k^{ \pm}\right\rangle}
$$

with $r^{\mu}$ a reference null momentum. One may show via the Fierz relations that these polarization vectors satisfy the axiomatic requirements $k \cdot \varepsilon^{ \pm}=0, \varepsilon^{ \pm} \cdot\left(\varepsilon^{ \pm}\right)^{*}=\varepsilon^{ \pm} \cdot \varepsilon^{\mp}=$ -1 and $\varepsilon^{ \pm} \cdot \varepsilon^{ \pm}=0$. In the present work, a particularly convenient choice of reference momenta is

$$
r_{1}=k_{2}, \quad r_{2}=k_{1}
$$

where $r_{1,2}\left(k_{1,2}\right)$ are reference momenta (photon momenta) corresponding to polarization vectors $\varepsilon_{1,2}^{ \pm}$defined in (5.7). We shall always make this choice of reference momenta.

The dotted and undotted Weyl spinors have explicit representations in terms of the momentum components in a particular basis, up to a free choice of phase. For example, for a null momentum $k=\left(k^{0}, k^{1}, k^{2}, k^{3}\right)$, a possible choice is

$$
\left(\lambda_{k}\right)^{a}= \begin{cases}\left(\frac{k^{1}+i k^{2}}{\sqrt{k^{0}-k^{3}}}, \sqrt{k^{0}-k^{3}}\right), & k^{3}<0, \\ \left(\sqrt{k^{0}+k^{3}}, \frac{k^{1}-i k^{2}}{\sqrt{k^{0}+k^{3}}}\right), & k^{3}>0 .\end{cases}
$$

The components of the upper dotted spinor are immediately specified through the relation $\left(\lambda_{k}\right)^{\dot{a}}=\left[\left(\lambda_{k}\right)^{a}\right]^{\dagger}$, and the lower index spinors through appropriate epsilon contractions. With this choice and a choice of basis, all spinor objects - e.g. $\langle k \ell\rangle-$ can be 
evaluated explicitly, just as momentum objects may be, e.g. $k \cdot \ell$. Note that for our Weyl spinor phase choice (C.6) one has $\left\langle k_{1} k_{2}\right\rangle=\left[k_{2} k_{1}\right]=m_{h}$.

The Dirac spinors of massive particles may also be represented in the spinor-helicity formalism, via the application of a light-cone decomposition. For a massive spinor of momentum $p$ and mass $m$, we define an associated null momentum

$$
\tilde{p}^{\mu}=p^{\mu}-\frac{m^{2}}{2 p \cdot \ell} \ell^{\mu}, \quad \text { or equivalently } \quad p^{\mu}=\tilde{p}^{\mu}+\frac{m^{2}}{2 \tilde{p} \cdot \ell} \ell^{\mu},
$$

where $\ell^{\mu}$ is a null reference momentum. The spinors then decompose similarly as

$$
\begin{aligned}
u^{1}(p) & =\left|\tilde{p}^{+}\right\rangle+\frac{m}{[\tilde{p} \ell]}\left|\ell^{-}\right\rangle, & u^{2}(p) & =\left|\tilde{p}^{-}\right\rangle-\frac{m}{\langle\ell \tilde{p}\rangle}\left|\ell^{+}\right\rangle, \\
\bar{u}^{1}(p) & =\left\langle\tilde{p}^{+}\right|+\frac{m}{\langle\ell \tilde{p}\rangle}\left\langle\ell^{-}\right|, & \bar{u}^{2}(p) & =\left\langle\tilde{p}^{-}\right|-\frac{m}{[\tilde{p} \ell]}\left\langle\ell^{+}\right|, \\
v^{1}(p) & =\left|\tilde{p}^{-}\right\rangle+\frac{m}{\langle\ell \tilde{p}\rangle}\left|\ell^{+}\right\rangle, & v^{2}(p) & =\left|\tilde{p}^{+}\right\rangle-\frac{m}{[\tilde{p} \ell]}\left|\ell^{-}\right\rangle, \\
\bar{v}^{1}(p) & =\left\langle\tilde{p}^{-}\right|+\frac{m}{[\tilde{p} \ell]}\left\langle\ell^{+}\right|, & \bar{v}^{2}(p) & =\left\langle\tilde{p}^{+}\right|-\frac{m}{\langle\ell \tilde{p}\rangle}\left\langle\ell^{-}\right| .
\end{aligned}
$$

One may verify that these spinors satisfy the canonical requirements $\bar{u} u=2 m, \bar{v} v=$ $-2 m, \bar{v} u=\bar{u} v=0$ and the completeness relations $\sum_{j} u^{j} \bar{u}^{j}=\not p+m$ and $\sum_{j} v^{j} \bar{v}^{j}=$ $\not p-m$. Just as for the polarization vectors, one is free to choose the null reference momentum. This choice amounts to a choice of 'gauge', under which the unpolarized square amplitude must be invariant, but the polarized square amplitudes are not. In this work, we shall always make the reference momenta choices

$$
\ell_{1_{ \pm}}=k_{2} \quad \text { and } \quad \ell_{2_{ \pm}}=k_{1} \text {. }
$$

These are convenient choices for the purposes of extracting the leading order $\mathrm{BH}$ helicity amplitudes, as below.

Finally, together with the several well-known spinor identities, the following iden- 
tity is especially useful for computing terms involving Levi-Civita contractions,

$$
\epsilon^{\alpha \beta \gamma \delta} \sigma_{\alpha}^{\dot{a} a} \sigma_{\beta}^{\dot{b} b} \sigma_{\gamma}^{\dot{c} c} \sigma_{\delta}^{\dot{d} d} \equiv 4 i\left(\epsilon^{\dot{d} \dot{a}} \epsilon^{b a} \epsilon^{\dot{b} \dot{c}} \epsilon^{d c}-\epsilon^{\dot{d} \dot{c}} \epsilon^{b c} \epsilon^{\dot{b} \dot{a}} \epsilon^{d a}\right)
$$

\section{C.II BH spin-helicity amplitudes}

Here we write down explicit expressions for the helicity amplitudes $\alpha_{i_{r s}}^{ \pm}$. From Eqs. (5.16) and (5.24) one has (suppressing the branch index)

$$
\begin{aligned}
& \alpha_{r s}^{ \pm}=-\frac{i}{2 q^{2}} \bar{u}^{r}\left(p_{-}\right)\left[\oiint^{ \pm} \frac{\not p_{-}-\not h+m}{k \cdot p_{-}} \not+\not \frac{\not k-\not p_{+}+m}{k \cdot p_{+}} \phi^{ \pm}\right] v^{s}\left(p_{+}\right) \\
& =-\frac{i}{2 q^{2}}\left[\sum_{j}\left(\frac{\bar{u}^{r} \ddagger^{ \pm} u^{j} \bar{u}^{j} \not v^{s}}{k \cdot p_{-}}-\frac{\bar{u}^{r} \phi v^{j} \bar{v}^{j} \ddagger^{ \pm} v^{s}}{k \cdot p_{+}}\right)-\frac{\bar{u}^{r} \ddagger^{ \pm} \not k \phi v^{s}}{k \cdot p_{-}}+\frac{\bar{u}^{r} \phi k \ddagger^{ \pm} v^{s}}{k \cdot p_{+}}\right] \text {, }
\end{aligned}
$$

where $u=u\left(p_{-}\right)$and $v=v\left(p_{+}\right)$. Applying the light cone decomposition (C.8) with reference choices specified in (C.4) and (C.9), gives the full results for each spin helicity 
amplitude

$$
\begin{aligned}
& \alpha_{11}^{-}=-\left(\alpha_{22}^{+}\right)^{*}=\frac{1}{q^{2} \sqrt{2}}\left[-\frac{m\left[\ell \tilde{p}_{-}\right]\left\langle k \tilde{p}_{-}\right\rangle\left\langle\ell^{-}|\not| \tilde{p}_{+}{ }^{-}\right\rangle}{k \cdot p_{-}[k \ell]\left\langle\ell \tilde{p}_{-}\right\rangle}+\frac{m\left[\ell \tilde{p}_{+}\right]\left\langle k \tilde{p}_{+}\right\rangle\left\langle\ell^{-}|\not| \tilde{p}_{+}{ }^{-}\right\rangle}{k \cdot p_{+}[k \ell]\left\langle\ell \tilde{p}_{-}\right\rangle}\right. \\
& -\frac{m\left[\ell \tilde{p}_{-}\right]\left\langle k \tilde{p}_{-}\right\rangle\left\langle\tilde{p}_{-}{ }^{+}|\phi| \ell^{+}\right\rangle}{k \cdot p_{-}[k \ell]\left\langle\ell \tilde{p}_{+}\right\rangle}+\frac{m\left[\ell \tilde{p}_{+}\right]\left\langle k \tilde{p}_{+}\right\rangle\left\langle\tilde{p}_{-}{ }^{+}|\phi| \ell^{+}\right\rangle}{k \cdot p_{+}[k \ell]\left\langle\ell \tilde{p}_{+}\right\rangle} \\
& \left.-\frac{m\langle k \ell\rangle\left\langle k^{-}|\not| \tilde{p}_{+}{ }^{-}\right\rangle}{k \cdot p_{-}\left\langle\ell \tilde{p}_{-}\right\rangle}+\frac{m\langle k \ell\rangle\left\langle\tilde{p}_{-}{ }^{+}|\not| k^{+}\right\rangle}{k \cdot p_{+}\left\langle\ell \tilde{p}_{+}\right\rangle}\right] \\
& \alpha_{12}^{-}=+\left(\alpha_{21}^{+}\right)^{*}=\frac{1}{q^{2} \sqrt{2}}\left[-\frac{m^{2}\langle k \ell\rangle\left\langle k^{-}|\not| \ell^{-}\right\rangle}{k \cdot p_{-}\left[\ell \tilde{p}_{+}\right]\left\langle\ell \tilde{p}_{-}\right\rangle}+\frac{m^{2}\left\langle k \tilde{p}_{+}\right\rangle\left\langle\ell^{-}|\not| \ell^{-}\right\rangle}{k \cdot p_{+}[k \ell]\left\langle\ell \tilde{p}_{-}\right\rangle}\right. \\
& -\frac{m^{2}\left[\ell \tilde{p}_{-}\right]\left\langle k \tilde{p}_{-}\right\rangle\left\langle\ell^{-}|\not| \ell^{-}\right\rangle}{k \cdot p_{-}[k \ell]\left[\ell \tilde{p}_{+}\right]\left\langle\ell \tilde{p}_{-}\right\rangle}-\frac{\left[\ell \tilde{p}_{-}\right]\left\langle k \tilde{p}_{-}\right\rangle\left\langle\tilde{p}_{-}{ }^{+}|\not| \tilde{p}_{+}{ }^{+}\right\rangle}{k \cdot p_{-}[k \ell]} \\
& \left.+\frac{\left[\ell \tilde{p}_{+}\right]\left\langle k \tilde{p}_{+}\right\rangle\left\langle\tilde{p}_{-}{ }^{+}|\not| \tilde{p}_{+}{ }^{+}\right\rangle}{k \cdot p_{+}[k \ell]}+\frac{\left\langle k \tilde{p}_{+}\right\rangle\left\langle\tilde{p}_{-}{ }^{+}|\not| k^{+}\right\rangle}{k \cdot p_{+}}\right] \\
& \alpha_{21}^{-}=+\left(\alpha_{12}^{+}\right)^{*}=\frac{1}{q^{2} \sqrt{2}}\left[-\frac{\left[k \tilde{p}_{-}\right]\left\langle k \tilde{p}_{-}\right\rangle\left\langle\tilde{p}_{-}^{-}|\not| \tilde{p}_{+}^{-}\right\rangle}{k \cdot p_{-}[k \ell]}+\frac{\left[\ell \tilde{p}_{+}\right]\left\langle k \tilde{p}_{+}\right\rangle\left\langle\tilde{p}_{-}^{-}|\not| \tilde{p}_{+}^{-}\right\rangle}{k \cdot p_{+}[k \ell]}\right. \\
& +\frac{m^{2}\langle k \ell\rangle\left\langle\ell^{+}|\not| k^{+}\right\rangle}{k \cdot p_{+}\left[\ell \tilde{p}_{-}\right]\left\langle\ell \tilde{p}_{+}\right\rangle}+\frac{m^{2}\left[\ell \tilde{p}_{+}\right]\left\langle k \tilde{p}_{+}\right\rangle\left\langle\ell^{+}|\not| \ell^{+}\right\rangle}{k \cdot p_{+}[k \ell]\left[\ell \tilde{p}_{-}\right]\left\langle\ell \tilde{p}_{+}\right\rangle} \\
& \left.-\frac{m^{2}\left\langle k \tilde{p}_{-}\right\rangle\left\langle\ell^{+}|\not| \ell^{+}\right\rangle}{k \cdot p_{-}[k \ell]\left\langle\ell \tilde{p}_{+}\right\rangle}-\frac{\left\langle k \tilde{p}_{-}\right\rangle\left\langle k^{-}|\not| \tilde{p}_{+}{ }^{-}\right\rangle}{k \cdot p_{-}}\right] \\
& \alpha_{22}^{-}=-\left(\alpha_{11}^{+}\right)^{*}=\frac{1}{q^{2} \sqrt{2}}\left[-\frac{m\left\langle k \tilde{p}_{-}\right\rangle\left\langle k^{-}|\not| \ell^{-}\right\rangle}{k \cdot p_{-}\left[\ell \tilde{p}_{+}\right]}+\frac{m\left\langle k \tilde{p}_{+}\right\rangle\left\langle\tilde{p}_{-}^{-}|\not| \ell^{-}\right\rangle}{k \cdot p_{+}[k \ell]}\right. \\
& -\frac{m\left[\ell \tilde{p}_{-}\right]\left\langle k \tilde{p}_{-}\right\rangle\left\langle\tilde{p}_{-}^{-}|\not| \ell^{-}\right\rangle}{k \cdot p_{-}[k \ell]\left[\ell \tilde{p}_{+}\right]}+\frac{m\left\langle k \tilde{p}_{+}\right\rangle\left\langle\ell^{+}|\not| k^{+}\right\rangle}{k \cdot p_{+}\left[\ell \tilde{p}_{-}\right]} \\
& \left.+\frac{m\left[\ell \tilde{p}_{+}\right]\left\langle k \tilde{p}_{+}\right\rangle\left\langle\ell^{+}|\not| \tilde{p}_{+}{ }^{+}\right\rangle}{k \cdot p_{+}[k \ell]\left[\ell \tilde{p}_{-}\right]}-\frac{m\left\langle k \tilde{p}_{-}\right\rangle\left\langle\ell^{+}|\not| \tilde{p}_{+}{ }^{+}\right\rangle}{k \cdot p_{-}[k \ell]}\right] .
\end{aligned}
$$

As per the main text, we have dropped the photon subscripts, and $k, \ell=k_{1}, k_{2}$ or $k_{2}, k_{1}$ for parent photon 1 and 2 respectively. Squaring these amplitudes, taking traces and summing, one obtains the full Bethe-Heitler square amplitude that is obtained by the usual Feynman methods.

We can further extract dominant terms of the $\mathrm{BH}$ spin-helicity amplitudes by observing that if $q_{i} \ll m_{h}$, then $\left\langle k_{1} k_{2}\right\rangle \gg\left\langle k_{i} p_{i_{ \pm}}\right\rangle$etc. Moreover, in expressions such as $\alpha_{12}^{+}$or $\alpha_{21}^{+}$, we may discard subdominant $\mathcal{O}\left(\mathrm{m}^{2} / k_{i} \cdot p_{j \neq i}\right)$ terms. This leads to the following leading order results in $m^{2} / k_{i} \cdot p_{j \neq i}$ and $\left\langle k_{i} p_{i}\right\rangle /\left\langle k_{1} k_{2}\right\rangle$ for the $\mathrm{BH}$ spin-helicity 
amplitudes

$$
\begin{aligned}
& \alpha_{11}^{-}=-\left(\alpha_{22}^{+}\right)^{*} \simeq \frac{m}{q^{2} \sqrt{2}}\left[\frac{\langle k \ell\rangle\left\langle\tilde{p}_{-}^{+}\left|\sigma^{0}\right| k^{+}\right\rangle}{k \cdot p_{+}\left\langle\ell \tilde{p}_{+}\right\rangle}-\frac{\langle k \ell\rangle\left\langle\tilde{p}_{+}^{+}\left|\sigma^{0}\right| k^{+}\right\rangle}{k \cdot p_{-}\left\langle\ell \tilde{p}_{-}\right\rangle}\right], \\
& \alpha_{12}^{-}=\left(\alpha_{21}^{+}\right)^{*} \simeq \frac{\left\langle k \tilde{p}_{+}\right\rangle\left\langle\tilde{p}_{-}^{+}\left|\sigma^{0}\right| k^{+}\right\rangle}{q^{2} \sqrt{2} k \cdot p_{+}}-\frac{\left[\ell \tilde{p}_{-}\right]\left\langle k \tilde{p}_{-}\right\rangle\left\langle\tilde{p}_{-}^{+}\left|\sigma^{0}\right| \tilde{p}_{+}^{+}\right\rangle}{q^{2} \sqrt{2} k \cdot p_{-}[k \ell]}+\frac{\left[\ell \tilde{p}_{+}\right]\left\langle k \tilde{p}_{+}\right\rangle\left\langle\tilde{p}_{-}^{+}\left|\sigma^{0}\right| \tilde{p}_{+}^{+}\right\rangle}{q^{2} \sqrt{2} k \cdot p_{+}[k \ell]} \\
& -\frac{m^{2}\langle k \ell\rangle\left\langle k^{-}\left|\sigma^{0}\right| \ell^{-}\right\rangle}{q^{2} \sqrt{2} k \cdot p_{-}\left[\ell \tilde{p}_{+}\right]\left\langle\ell \tilde{p}_{-}\right\rangle}, \\
& \alpha_{21}^{-}=\left(\alpha_{12}^{+}\right)^{*} \simeq-\frac{\left\langle k \tilde{p}_{-}\right\rangle\left\langle\tilde{p}_{+}^{+}\left|\sigma^{0}\right| k^{+}\right\rangle}{q^{2} \sqrt{2} k \cdot p_{-}}-\frac{\left[\ell \tilde{p}_{-}\right]\left\langle k \tilde{p}_{-}\right\rangle\left\langle\tilde{p}_{-}^{-}\left|\sigma^{0}\right| \tilde{p}_{+}^{-}\right\rangle}{q^{2} \sqrt{2} k \cdot p_{-}[k \ell]}+\frac{\left[\ell \tilde{p}_{+}\right]\left\langle k \tilde{p}_{+}\right\rangle\left\langle\tilde{p}_{-}^{-}\left|\sigma^{0}\right| \tilde{p}_{+}^{-}\right\rangle}{q^{2} \sqrt{2} k \cdot p_{+}[k \ell]} \\
& +\frac{m^{2}\langle k \ell\rangle\left\langle k^{-}\left|\sigma^{0}\right| \ell^{-}\right\rangle}{q^{2} \sqrt{2} k \cdot p_{+}\left[\ell \tilde{p}_{-}\right]\left\langle\ell \tilde{p}_{+}\right\rangle}, \\
& \alpha_{22}^{-}=-\left(\alpha_{11}^{+}\right)^{*} \simeq 0,
\end{aligned}
$$

in which we have dropped the photon subscripts, and uniform overall signs or factors of $i ; k, \ell=k_{1}, k_{2}$ or $k_{2}, k_{1}$ for photon 1 and 2 respectively; and the spinor notation is detailed in Appendix C.I. The parity relations (5.29) are satisfied as expected. Eqs. (5.28) and (C.13) together provide a compact expression of the leading order $\mathrm{HBH}$ square amplitude.

It should be understood that the particular form for the spin helicity amplitudes above depends on the choice of reference momenta, because the amplitudes explicitly depend on polarization vectors and spinors (see App. C.I). Moreover, the ability to straightforwardly expand the full results to the leading order results depends on a sensible choice of reference momenta. In contrast, the full unpolarized $\mathrm{BH}$ rate is independent of polarizations and spinors, as a result of spinor and polarization vector completeness, and therefore must be independent of any such reference momenta choice. 


\section{C.III Polarization-decomposed $\mathrm{HBH}$ rate}

Here we give the explicit results of a Higgs-Bethe-Heitler Feynman type calculation. To preempt the loss of numerical precision from large cancellations due to the Ward identity, we do not use polarization completeness relations. Rather, we retain the polarization vectors explicitly in the $\mathrm{HBH}$ rate. In the case that the Higgs is at rest in

the lab frame, we simply use a Cartesian basis for the polarization vectors, aligning the back-to-back photons with the $z$-axis. That is, in the HBH square amplitude we coherently sum over the polarization basis

$$
\epsilon_{\mu}^{1}\left(k_{1,2}\right)=(0,1,0,0) \quad \text { and } \quad \epsilon_{\mu}^{2}\left(k_{1,2}\right)=(0,0,1,0)
$$

The squared matrix element for the process $h+N_{1}+N_{2} \rightarrow \gamma\left(k_{1}\right) \gamma\left(k_{2}\right)+N_{1}+N_{2} \rightarrow$ $4 \ell+N_{1}^{\prime}+N_{2}^{\prime}$ is given by

$$
|\mathcal{M}|^{2}=\mathcal{A}^{\mu \nu} \mathcal{A}^{* \alpha \beta} \sum_{\substack{\text { pols } \\ \text { a,b,c,d }}}\left[\epsilon_{\mu}^{a *}\left(k_{1}\right) \epsilon_{\nu}^{b *}\left(k_{2}\right) \epsilon_{\alpha}^{c}\left(k_{1}\right) \epsilon_{\beta}^{d}\left(k_{2}\right) \epsilon_{\mu^{\prime}}^{a}\left(k_{1}\right) \epsilon_{\alpha^{\prime}}^{c *}\left(k_{1}\right) \epsilon_{\nu^{\prime}}^{b}\left(k_{2}\right) \epsilon_{\beta^{\prime}}^{d *}\left(k_{2}\right)\right] \mathrm{BH}_{1}^{\mu^{\prime} \alpha^{\prime}} \mathrm{BH}_{2}^{\nu^{\prime} \beta^{\prime}}
$$

where the tensor $\mathcal{A}^{\mu \nu}$ is

$$
\mathcal{A}^{\mu \nu}=c\left(k_{1} \cdot k_{2} g^{\mu \nu}-k_{2}^{\mu} k_{1}^{\nu}\right)+\tilde{c} \epsilon^{\alpha \mu \beta \nu} k_{1 \alpha} k_{2 \beta} .
$$

The $\mathrm{BH}_{i}$ factors are the polarized Bethe-Heitler squared amplitudes for a photon $i$, 
including form factor contributions, these are in general

$$
\begin{aligned}
\mathrm{BH}^{a b} & =\frac{\mathcal{G}}{q^{4}\left(k \cdot p_{-}\right)^{2}\left(k \cdot p_{+}\right)^{2}}\left\{\left[2\left(k \cdot p_{-}\right)\left(k \cdot p_{+}\right)\left(2 E_{-} E_{+}-m^{2}-p_{+} \cdot p_{-}\right) \mathcal{B}_{k, p_{-}}^{a b}\right.\right. \\
& \left.+\frac{8 E_{+}}{M}\left(k \cdot p_{+}\right)^{2}\left(k \cdot p_{-}\right) \mathcal{B}_{P, p_{-}}^{a b}+\left(k \cdot p_{+}\right)^{2}\left(4 E_{+}^{2}+q^{2}\right) \mathcal{F}_{p_{-}}^{a b}+\left(p_{+} \leftrightharpoons p_{-}\right)\right] \\
& -8\left(k \cdot p_{-}\right)^{2}\left(k \cdot p_{+}\right)^{2} \frac{P^{a} P^{b}}{M^{2}} \\
& -\frac{2}{M}\left(k \cdot p_{-}\right)\left(k \cdot p_{+}\right)\left[-q^{2}+2\left(E_{-}\left(k \cdot p_{-}\right)+E_{+}\left(k \cdot p_{+}\right)\right)\right] \mathcal{B}_{P, k}^{a b} \\
& +2\left(k \cdot p_{-}\right)\left(k \cdot p_{+}\right)\left(q^{2}-4 E_{-} E_{+}\right) \mathcal{B}_{p_{+}, p_{-}}^{a b} \\
& +2\left(k \cdot p_{-}\right)\left(k \cdot p_{+}\right)\left[\left(k \cdot p_{-}+k \cdot p_{+}\right)^{2}+q^{2} E_{\gamma}^{2}\right] g^{a b} \\
& \left.-4\left(k \cdot p_{-}\right)\left(k \cdot p_{+}\right)\left(2 E_{-} E_{+}-m^{2}-p \cdot r\right) k^{a} k^{b}\right\}
\end{aligned}
$$

where $\mathcal{F}_{\ell}^{a b}=\ell^{a} k^{b}+\ell^{b} k^{a}-2 \ell^{a} \ell^{b}$ and $\mathcal{B}_{\ell_{1} \ell_{2}}^{a b}=\ell_{1}^{a} \ell_{2}^{b}+\ell_{1}^{b} \ell_{2}^{a}$. The photon momentum is denoted by $k$ and the lepton momenta by $p_{+}, p_{-} . M$ and $m$ are the masses of the nucleus and lepton respectively. Assuming that the Higgs is at rest in the lab frame and that the photon is in the $z$-direction, as in Eq. (C.14), the previous expression simplifies to $\mathrm{BH}^{a b} \simeq \frac{2 \mathcal{G}}{q^{4}}\left\{\frac{g^{a b}\left[E_{\gamma}^{2} q^{2}+\left(k \cdot p_{-}+k \cdot p_{+}\right)^{2}\right]}{\left(k \cdot p_{-}\right)\left(k \cdot p_{+}\right)}-4\left(\frac{E_{p_{+}} p_{-}^{a}}{k \cdot p_{-}}+\frac{E_{p_{-}} p_{+}^{a}}{k \cdot p_{+}}\right)\left(\frac{E_{p_{+}} p_{-}^{b}}{k \cdot p_{-}}+\frac{E_{p_{-}} p_{+}^{b}}{k \cdot p_{+}}\right)\right\}$,

where, in the second term, we expanded terms of the form $\sqrt{4 E^{2} \pm q^{2}}$ to leading order in $q^{2} / E^{2}$. We have checked that the helicity formalism results and the Feynman diagram calculation results for $\mathrm{HBH}$ rate agree.

Finally, the unpolarized BH rate can be obtained from Eq. (C.18) simply by averaging over the photon polarization as follows

$$
\left\langle|\mathcal{M}|^{2}\right\rangle=1 / 2\left(\mathrm{BH}^{11}+\mathrm{BH}^{22}\right)
$$

It is instructive to use the polarization vector completeness relation to obtain an ex- 
pression in terms of Lorentz dot products. In this case, starting with Eq. (C.17), the BH rate is given by

$$
\begin{aligned}
\left\langle|\mathcal{M}|^{2}\right\rangle= & -4 \frac{\mathcal{G}\left(E_{-}^{2}+E_{+}^{2}\right)}{q^{2} k \cdot p_{-} k \cdot p_{+}}+8 \frac{\mathcal{G} m^{2}\left(k \cdot p_{-} E_{-}-k \cdot p_{+} E_{+}\right)^{2}}{q^{4}\left(k \cdot p_{-}\right)^{2}\left(k \cdot p_{+}\right)^{2}} \\
& +2 \frac{\mathcal{G} m^{2}\left(k \cdot p_{-}+k \cdot p_{+}\right)^{2}}{q^{2}\left(k \cdot p_{-}\right)^{2}\left(k \cdot p_{+}\right)^{2}}-2 \frac{\mathcal{G} k \cdot p_{-} k \cdot p_{+}}{\left(k \cdot p_{-}\right)^{2}\left(k \cdot p_{+}\right)^{2}}-4 \frac{\mathcal{G}\left(k \cdot p_{-}\right)^{2}+\left(k \cdot p_{+}\right)^{2}}{q^{4}\left(k \cdot p_{-}\right)\left(k \cdot p_{+}\right)} \\
& -4 \frac{\mathcal{G} k \cdot p_{-}+k \cdot p_{+}}{q^{2} k \cdot p_{-} k \cdot p_{+}}
\end{aligned}
$$

where the leading terms - the terms on the first line - reproduce Eq. (5.35).

\section{C.IV Numerical simulations of Bethe-Heitler conversion}

In this appendix we present numerical evaluations of several differential $\mathrm{BH}$ rates. The numerics were done in two ways: by numerically integrating the full tree-level analytical results - i.e. the $\mathrm{BH}$ rate arising from Eqs. (C.12) or (C.17) with appropriate integration measures - using the CUBA library [313]; and with a Monte Carlo (MC) code developed privately (the details are given in Appendix C.VI). Fig. C.1 shows the differential distribution of the positron energy fraction $\mathcal{E}_{+}=E_{+} / E_{\gamma}$. For efficiency the MC simulation (blue binned histogram) is generated with a cut on the difference of electron and positron azimuthal angles, $\delta \phi \equiv\left(\phi_{+}-\phi_{-}\right) \bmod 2 \pi \in[0.6 \pi, 1.4 \pi]$ (see App. C.VI). This agrees with the numerical integration (red line) for the same cuts on $\delta \phi$.

Fig. C.1 (right) shows the positron energy distribution after applying the opening angle cut of $\theta_{\ell \ell}>10^{-4}$ on the angle between $e^{+}$and $e^{-}$momenta. The MC agrees with the full numerical integration of the $\mathrm{BH}$ rate even though the $\delta \phi$ cut is still applied in the generation of the events . Fig. C.1 demonstrates that the asymmetric configurations, where one of the two leptons carries the larger part of the photon energies, are the more 

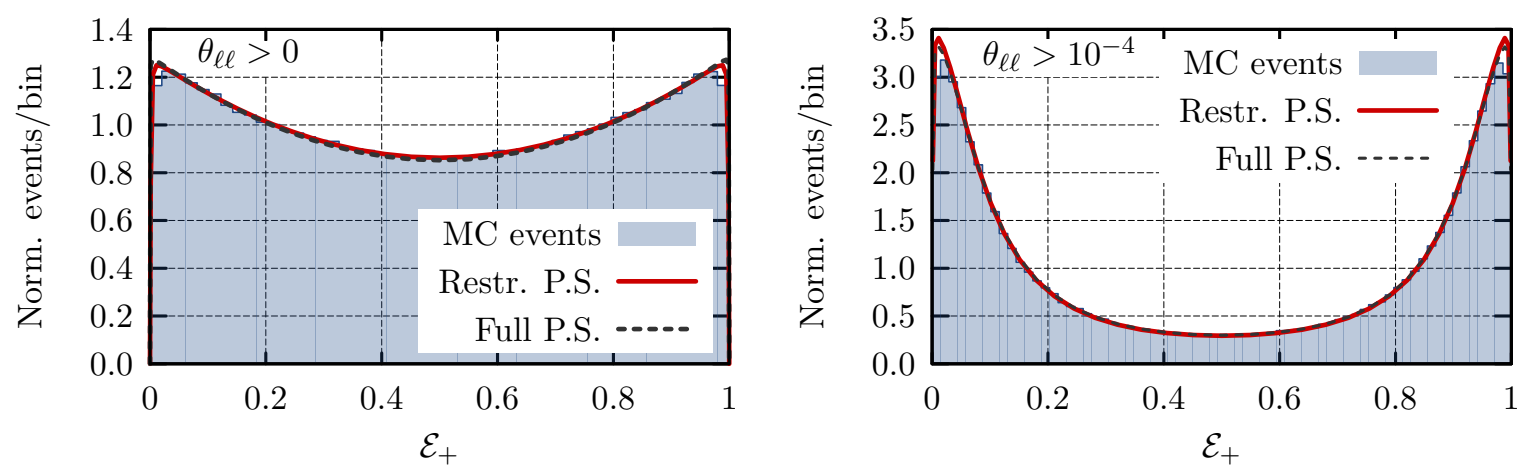

Figure C.1: Spectrum of the positron energy $\mathcal{E}_{+}=E_{+} / E_{\gamma}$. No opening angle cut was applied in the left hand figure and an opening angle cut of $10^{-4}$ was applied in the right hand one. The histograms were created with MC events and the solid curves are results of numerically integrating the differential cross section. The dashed curve in both figures is the result of numerically integrating the differential cross section over the entire range of $\delta \phi$ as opposed over the range $[0.6 \pi, 1.4 \pi]$.

probable ones, especially for non-zero opening angles.

Fig. C.2 shows the positron polar angle distribution and demonstrates the combined effect of the Si nuclear form factor and the smaller available phase space that suppress very small momentum transfers and thus very small polar angles. The peak is at $\sim m / E \sim 10^{-5}$ both for the distribution without a cut on the $e^{+} e^{-}$opening angle $\theta_{\ell \ell}$, Fig. C.2 (left), and for the case where $\theta_{\ell \ell}>10^{-4}$ is imposed, Fig. C.2 (right). This cut also results in an additional peak in the distribution, cf. Fig. C.2 (right).

In Fig. C.3, the distribution in the relative azimuthal angle $\delta \phi$ of the two leptons is shown. The majority of the events are close to the coplanar configuration, where the photon and the two lepton momenta all lie in the same plane. However, it is noteworthy that approximately $40 \%$ of $\mathrm{BH}$ events have acoplanarity of $\sim 5 \%$ or more.

\section{C.V Analysis for $q \bar{q} \rightarrow \gamma \gamma$}

Performing the measurement proposed in this work faces two main challenges: first, resolving and reconstructing the electron and positron directions; and second identify- 

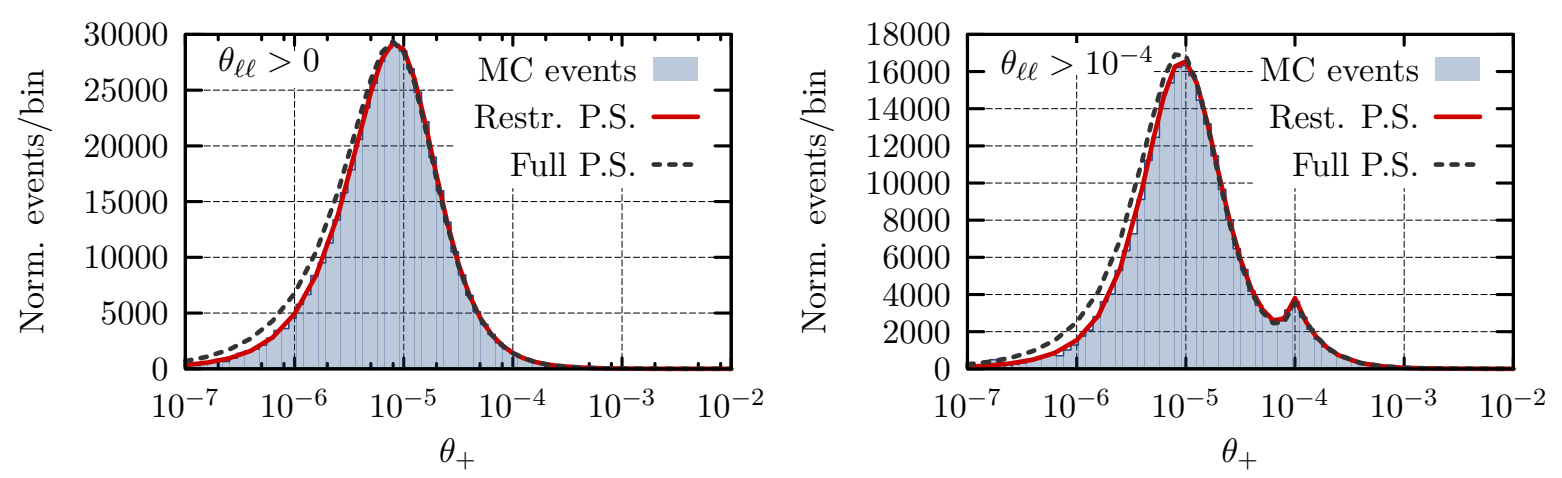

Figure C.2: Polar angle distribution of the leptons. No opening angle cut was applied in the left hand figure and an opening angle cut of $10^{-4}$ was applied in the right hand one. The histograms were created with MC events and the solid curves are results of numerically integrating the differential rate expression. The small bump in the right hand figure $\left(\sim 10^{-4}\right)$ is a result of applying an opening angle cut. Its location is a function of the cut.

ing a background-poor sample of events with Higgs decaying to diphotons. Regarding the first challenge, one might simply ask how well and with what efficiency can the LHC or a future collider detector reconstruct the details of photon conversion. To do so, the experimental collaborations may wish to test the polarization structure of a standard model (non-Higgs) amplitude. To demonstrate that there is a non-trivial structure to be measured in SM conversions, we briefly analyze here the leading production of diphotons at the LHC.

The dominant diphoton production (and dominant background for Higgs to photons events) is $q \bar{q} \rightarrow \gamma \gamma$ scattering. This has tree-level spin-helicity amplitude

$$
\left[\mathcal{M}_{\mathrm{BG}}\right]_{r s}^{\lambda_{1} \lambda_{2}}=P_{P_{-}, r \longrightarrow k_{1}, \lambda_{1}}^{P_{+}, s \longrightarrow k_{2}, \lambda_{2}}+\text { u channel, }
$$

in which $r=1,2(s=1,2)$ is the spin of the (anti)-fermion, and $\lambda_{1,2}= \pm$ are the usual photon helicities. The two photons then convert in the tracker. The background rate 

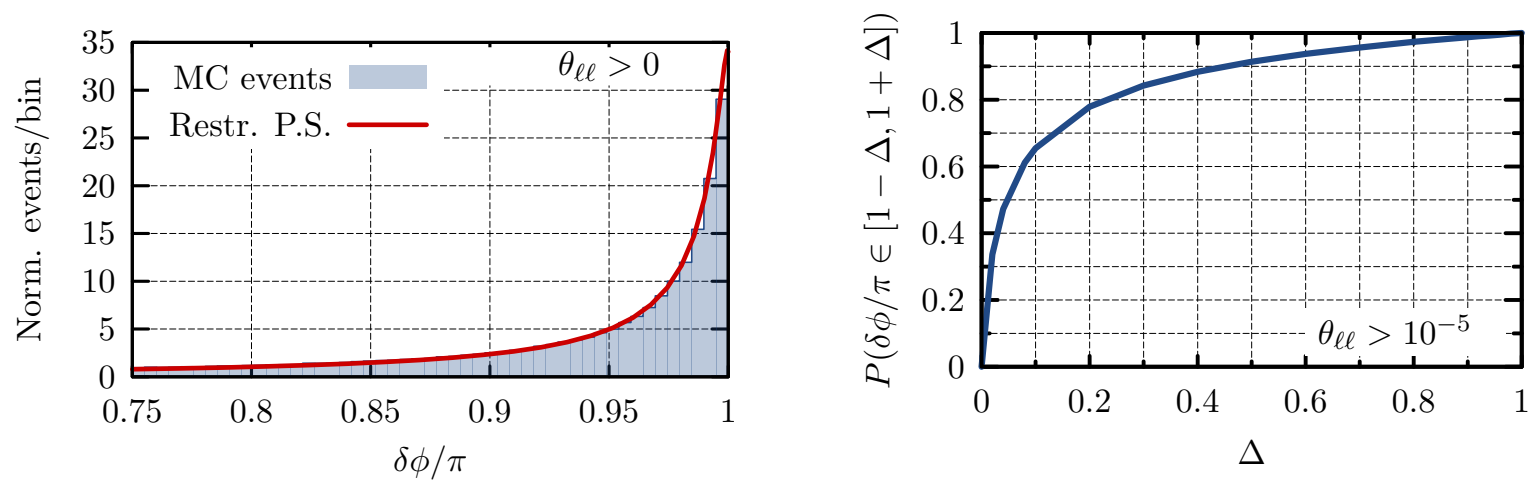

Figure C.3: Left: Distribution of the azimuthal angle between the leptons. The histograms were created with MC events and the solid curves are results of numerically integrating the differential rate expression. Right: The cumulative distribution function of the relative azimuthal orientation, $P(\delta \phi / \pi \in[1-\Delta, 1+\Delta])$, from numerical integration.

can be written in a form similar to Eq. (5.28). That is, for one fermion species

$$
\left|\mathcal{M}_{\mathrm{BG}}\right|^{2}=\mathcal{G}_{1} \mathcal{G}_{2} \sum_{r, s} \sum_{r_{1}, s_{1}, r_{2}, s_{2}}\left|\sum_{\lambda_{1}, \lambda_{2}}\left[\mathcal{M}_{\mathrm{BG}}\right]_{r s}^{\lambda_{1} \lambda_{2}} \alpha_{1_{r s}}^{\lambda_{1}} \alpha_{2_{r s}}^{\lambda_{2}}\right|^{2}
$$

As is well-known, the only non-zero independent amplitudes are $\left[\mathcal{M}_{\mathrm{BG}}\right]_{12}^{+-}$and $\left[\mathcal{M}_{\mathrm{BG}}\right]_{12}^{-+}$. One finds with our usual choice of reference momenta, and the light cone decomposition (C.8) for the quark momenta $P_{ \pm}$,

$$
\begin{aligned}
{\left[\mathcal{M}_{\mathrm{BG}}\right]_{12}^{+-}=\left(\left[\mathcal{M}_{\mathrm{BG}}\right]_{21}^{-+}\right)^{*} } & =\frac{\mathcal{A}_{+}\left(k_{2}, k_{1}\right)}{P_{+} \cdot k_{1}}-\frac{\mathcal{A}_{-}^{*}\left(k_{1}, k_{2}\right)}{P_{-} \cdot k_{1}}, \\
{\left[\mathcal{M}_{\mathrm{BG}}\right]_{12}^{-+}=\left(\left[\mathcal{M}_{\mathrm{BG}}\right]_{21}^{+-}\right)^{*} } & =\frac{\mathcal{A}_{+}\left(k_{1}, k_{2}\right)}{P_{+} \cdot k_{1}}-\frac{\mathcal{A}_{-}^{*}\left(k_{2}, k_{1}\right)}{P_{-} \cdot k_{1}} \\
\mathcal{A}_{ \pm}(k, \ell) & =Q_{f}^{2} \frac{\left\langle\tilde{P}_{ \pm} \ell\right\rangle^{2}\left[\tilde{P}_{ \pm} k\right]\left[\tilde{P}_{\mp} k\right]}{2 k_{1} \cdot k_{2}}
\end{aligned}
$$

with $Q_{f}$ the fermion electric charge. The square amplitude simplifies to

$$
\left|\mathcal{M}_{\mathrm{BG}}\right|^{2}=2 \mathcal{G}_{1} \mathcal{G}_{2} \sum_{r_{1}, s_{1}, r_{2}, s_{2}}\left|\left[\mathcal{M}_{\mathrm{BG}}\right]_{12}^{+-} \alpha_{1_{r s}}^{+} \alpha_{2 r s}^{-}+\left[\mathcal{M}_{\mathrm{BG}}\right]_{12}^{-+} \alpha_{1_{r s}}^{-} \alpha_{2_{r s}}^{+}\right|^{2}
$$

It is interesting to contrast this with the $\mathrm{HBH}$ result. Here the helicity interference arises 
in terms of the form $\alpha_{1_{12}}^{-} \alpha_{2_{12}}^{+} \alpha_{1_{21}}^{-} \alpha_{2_{21}}^{+}$, rather than from $\alpha_{1_{12}}^{-} \alpha_{2_{12}}^{-} \alpha_{1_{21}}^{-} \alpha_{2_{21}}^{-}$as we found for HBH. Since $\alpha_{12}^{+}=\left(\alpha_{21}^{-}\right)^{*}$, etc, it follows from the explicit results (5.36) that this helicity flip on branch ' 2 ' produces a phase change $\phi_{2_{ \pm}} \rightarrow-\phi_{2_{ \pm}}$in the background interference term, compared to the $\mathrm{HBH}$ interference terms.

To compare with the Higgs rest frame HBH rate (5.48), we assume the quark centre of mass frame aligns with the nuclear rest frame. Integrating all over azimuthal structure except $\psi \equiv \phi_{1_{+}}-\phi_{2_{+}}$(cf. $\varphi=\phi_{1_{+}}+\phi_{2_{+}}$in Eq. (5.46)), one finds that the background rate has the form

$$
\frac{d \Gamma}{d \psi d \mathrm{PS}_{\gamma, \theta}}=\mathcal{A}_{\gamma, \theta}^{\mathrm{BG}}+\mathcal{B}_{\gamma, \theta}^{\mathrm{BG}} \cos (2 \psi) .
$$

We see that this differential rate has sinusoidal dependence on the mean azimuthal orientation, $\psi$, of the outgoing positrons, with respect to, say, the incoming quarks the beamline - rather than the inter-branch lepton azimuthal orientation, $\varphi$, as in $\mathrm{HBH}$. Moreover, the phase change $\phi_{2 \pm} \rightarrow-\phi_{2_{ \pm}}$ensures background is flat in $\varphi$. Note also that unlike the $\mathrm{HBH}$ process, the $\left[\mathcal{M}_{\mathrm{BG}}\right]^{+-}$factors ensure this background rate features higher spin waves, so that its angular differential structure will differ from the $\mathrm{HBH}$ structure, too.

In summary, the leading-order doubly-converted $q \bar{q} \rightarrow \gamma \gamma$ square amplitude is given explicitly by Eqs. (C.23) and (C.24) combined with the BH spin-helicity amplitudes (C.13). In the $q \bar{q}$ center of mass frame, the corresponding leading order $\mathrm{BH}$ amplitudes are given in Eqs. (5.36).

\section{C.VI Monte Carlo numerical schemes}

To generate Monte Carlo (MC) events for the HBH process, we first generate unpolarized $\mathrm{BH}$ events and then use the von Neumann rejection technique to re-weight the events according to the $\mathrm{HBH}$ differential rate. For a single $\mathrm{HBH}$ event one needs two 
$\mathrm{BH}$ events taken from disjoint MC samples. Therefore, we first describe the generation of unpolarized BH events.

The phase space for a single $\mathrm{BH}$ event, $\gamma N \rightarrow e^{+} e^{-} N$, is five dimensional. We take $z$-axis to be the incoming photon direction. For conversion of unpolarized photons, the kinematics are invariant under overall azimuthal rotations around the $z$ axis. We therefore fix the positron azimuthal angle to zero. The remaining four coordinates are chosen to be the electron energy fraction $\mathcal{E}_{-}=E_{-} / E_{\gamma}$, two transformed polar angles, $t_{1}=\log _{10}\left(\theta_{+}\right)$and $t_{2}=\log _{10}\left(\theta_{-}\right)$, and the azimuthal angle of the electron $\phi_{-}$(see Fig. 5.6 for definitions).

For MC we used two independent private codes. One is written in C and the other in $\mathrm{C}++^{+} \mathrm{Java}$. To populate the $\mathrm{BH}$ phase space we first randomly generate the values for $\mathcal{E}_{-}, t_{1,2}$ and $\phi_{-}$according to either uniform distributions or conveniently chosen initial probability density functions (PDFs) and then unweight to obtain the $\mathrm{BH}$ event distribution.

For the C code, the initial PDFs are as follows. For a $60 \mathrm{GeV}$ photon, generating $\mathcal{E}_{-}$according to a uniform distribution results in an efficiency of $\sim 70 \%$. We therefore generate a uniform distribution of $\mathcal{E}_{-} \in\left[m / E_{\gamma}, 1-m / E_{\gamma}\right]$. The transformed polar angle variables, $t_{1}$ and $t_{2}$, are generated according to uniform distributions in a suitable numerical range, see Table C.1. Using the coordinates $t_{1,2}$ captures the fact that electron and positron distributions are sharply peaked around $\theta_{ \pm} \sim m / E$. The $\mathrm{BH}$ events are also dominated by kinematic configurations that are not too far from the coplanar one. We therefore generate $\phi_{-}$in the range $[0.6 \pi, 1.4 \pi]$, which suffices for our precision. To capture the fact that the $\mathrm{BH}$ distribution is peaked toward $\phi_{-} / \pi=1$, the $\mathrm{C}$ code generates $\phi_{-}$according to a Cauchy distribution (Lorentzian) with location parameter $x_{o}=\pi$ and scale parameter $\lambda=0.03 \pi$ [314] to improve the efficiency. The $\mathrm{C}++/$ Java uses similar initial data, with the exception of slightly different ranges and that $\phi_{-}$is populated by a uniform distribution on a slightly narrowed domain $\phi_{-} \in[3 \pi / 4,5 \pi / 4]$ 


\begin{tabular}{ccccc}
\hline & \multicolumn{2}{c}{$\mathrm{C}$} & \multicolumn{2}{c}{ C++/Java } \\
Parameter & Range & PDF & Range & PDF \\
\hline $\mathcal{E}_{-}$ & {$\left[m / E_{\gamma}, 1-m / E_{\gamma}\right]$} & Uniform & {$\left[m / E_{\gamma}, 1-m / E_{\gamma}\right]$} & Uniform \\
$t_{1}$ & {$[-7,-2]$} & Uniform & {$[-6,-3]$} & Uniform \\
$t_{2}$ & {$[-7,-2]$} & Uniform & {$[-6,-3]$} & Uniform \\
$\phi_{-}$ & {$[0.6 \pi, 1.4 \pi]$} & Lorentzian & {$[3 \pi / 4,5 \pi / 4]$} & Uniform \\
\hline \hline
\end{tabular}

Table C.1: The details on the MC generation of BH events, with phase space variables (1st column) for C ( $\mathrm{C}++/ \mathrm{Java})$ generated in the range given in the 2nd (4th) column according to the distribution given in the 3 rd (5th) column (for details see text).

(see Table. C.1).

In the next step we unweight the events generated from initial PDFs to obtain the proper $\mathrm{BH}$ distribution. In the unweighting, the events are rejected with a probability that is $1-w$, with $w=\left(d \Gamma_{\mathrm{BH}} / d \mathrm{PS}\right) / \max \left(d \Gamma_{\mathrm{BH}} / d \mathrm{PS}\right)$. The $\mathrm{BH}$ MC event sample was validated by comparing the generated event distributions to the results of numerical integration of BH differential cross-sections as shown in Figs. C.1, C.2 and C.3. Additionally, in Figs. C.1 and C.2, the distributions with a cut on the opening angle between $e^{+}$and $e^{-}$of $\theta_{\ell \ell}>10^{-4}$ are shown. The MC sample is in excellent agreement with the results of numerical integration.

In the final step, we convert the generated $\mathrm{BH}$ events into $\mathrm{MC}$ event samples for the $\mathrm{HBH}$ process. To do so, two disjoint $\mathrm{BH}$ samples were used - one sample per photon branch. The rate for two $\mathrm{BH}$ events is given by $\left(d \Gamma_{\mathrm{BH}_{1}} / d \mathrm{PS}\right)\left(d \Gamma_{\mathrm{BH}_{2}} / d \mathrm{PS}\right)$. To obtain the proper $\mathrm{HBH}$ even rates, we use the standard reweighting technique where events are rejected according to the weight

$$
w=\frac{\left(d \Gamma_{\mathrm{HBH}} / d \mathrm{PS}\right)[\varphi]}{\left(d \Gamma_{\mathrm{BH}_{1}} / d \mathrm{PS}\right)\left(d \Gamma_{\mathrm{BH}_{2}} / d \mathrm{PS}\right)},
$$

where the twist angle between the positrons, $\varphi$, is populated by a uniform distribution on $[0,2 \pi]$. 
The two MC codes have been cross tested. In numerics we use $3 \times 10^{6} \mathrm{HBH}$ events from the $\mathrm{C}$ generator and $8 \times 10^{5} \mathrm{HBH}$ events from the $\mathrm{C}++/$ Java $\mathrm{MC}$ generator. 


\section{D - Appendix to Chapter 6}

\section{D.I The $B \rightarrow K \pi e^{+} e^{-}$squared matrix element by polar- ization decomposition}

The effective Lagrangian in eq. (6.2) gives the following Feynman rule for the $B K^{*} \gamma$ vertex:

$$
\cdots \sim_{\tau_{K^{*}(p)^{\kappa}, \nu}}^{\gamma(k)^{\lambda}, \mu}=i\left[g_{\|}\left(\Delta m_{B K^{*}}^{2} g_{\mu \nu}-2 k_{\nu} p_{\mu}\right)+2 g_{\perp} \epsilon_{\mu \nu \rho \sigma} k^{\rho} p^{\sigma}\right]
$$

where $\Delta m_{B K^{*}}^{2} \equiv m_{B}^{2}-m_{K^{*}}^{2}$. The amplitude for $B \rightarrow \gamma^{\lambda} K \pi$ with $\lambda$ being the photon helicity is then given by

$$
\begin{gathered}
\mathcal{N}_{\lambda}=g_{K^{*}}\left\{g_{\|}\left[\Delta m_{B K^{*}}^{2} \varepsilon_{\lambda}^{*} \cdot\left(p_{K}-p_{\pi}\right)-2 \varepsilon_{\lambda}^{*} \cdot p\left[k \cdot\left(p_{K}-p_{\pi}\right)\right]\right]\right. \\
\left.-2 g_{\perp} \epsilon^{\mu \nu \rho \sigma}\left(p_{K}-p_{\pi}\right)_{\mu} \varepsilon_{\nu}^{\lambda *} k_{\rho} p_{\sigma}\right\}
\end{gathered}
$$

The BH squared amplitude in the nuclear rest frame for a linearly polarized photon in the $+\hat{z}$ direction with polarizations $\lambda=\{1,2\}$ is

$\mathrm{BH}^{\lambda \lambda^{\prime}} \simeq \frac{2 e^{6} \mathcal{G}}{q^{4}}\left\{\frac{g^{\lambda \lambda^{\prime}}\left[E_{\gamma}^{2} q^{2}+\left(k \cdot p_{-}+k \cdot p_{+}\right)^{2}\right]}{\left(k \cdot p_{-}\right)\left(k \cdot p_{+}\right)}-4\left(\frac{E_{p_{+}} p_{-}^{\lambda}}{k \cdot p_{-}}+\frac{E_{p_{-}} p_{+}^{\lambda}}{k \cdot p_{+}}\right)\left(\frac{E_{p_{+}} p_{-}^{\lambda^{\prime}}}{k \cdot p_{-}}+\frac{E_{p_{-}} p_{+}^{\lambda^{\prime}}}{k \cdot p_{+}}\right)\right\}$ 
where terms of $\mathcal{O}\left(q^{2} / E_{ \pm}^{2}\right)$ were dropped (see Appendix C in [299] for details). The squared amplitude is then given by

$$
|\mathcal{M}|^{2}=\sum_{\lambda, \lambda^{\prime} \in\{1,2\}} \mathcal{N}_{\lambda} \mathcal{N}_{\lambda^{\prime}}^{*} \mathrm{BH}^{\lambda \lambda^{\prime}}
$$

A numerical comparison between the above expression and eq. (6.23) shows excellent agreement over the entire phase space (sampled uniformly).

\section{D.II $B$ rapidity distribution}

Consider an $e^{+} e^{-} \rightarrow \Upsilon \rightarrow B \bar{B}$ factory, and let $\Theta$ denote the polar angle of the $B^{\prime} \mathrm{s}$ with respect to the electron beamline in the center of mass frame. The amplitude for production $\mathcal{M}_{\text {prod }} \sim \sin \Theta$, and so the probability distribution

$$
p_{\Omega}(\cos \Theta)=\frac{3}{4}\left[1-\cos ^{2} \Theta\right]
$$

Here and in the following we neglect effects of lab frame angular acceptance cuts, which may non-trivially restrict the domain of both $\eta$ and $\Theta$.

In the center of mass frame - the rest frame of the $\Upsilon$ - each $B$ has energy $E^{*}=m_{\Upsilon} / 2$ : hereafter the * superscript denotes center-of-mass frame quantities. The corresponding rapidity, which we choose to be positive by convention on the branch $\Theta \in[0, \pi]$,

$$
\eta^{*}=\cosh ^{-1}\left(m_{\Upsilon} / 2 m_{B}\right),
$$

and the $B$ speed in this frame $\beta^{*}=\tanh \left(\eta^{*}\right)$.

At $B$-factories the lab frame electron and position beam energies, $\mathcal{E}_{ \pm}$, are asymmetric, but are chosen such that the $\Upsilon$ is on shell, i.e. $4 \mathcal{E}_{+} \mathcal{E}_{-}=m_{\Upsilon}^{2}$. For example, at Belle II the beams are planned to be $\mathcal{E}_{+}=7 \mathrm{GeV}$ and $\mathcal{E}_{-}=4 \mathrm{GeV}$. The boost rapidity of the 
center of mass frame with respect to the lab frame is correspondingly

$$
\eta_{\Upsilon}=\cosh ^{-1}\left[\left(\mathcal{E}_{+}+\mathcal{E}_{-}\right) / m_{\Upsilon}\right]
$$

The rapidity of the $B$ in the lab frame may now be written as a function of $\cos \Theta$, viz.

$$
\eta(\cos \Theta)=\cosh ^{-1}\left\{\cosh \eta^{*}\left[\cosh \eta_{\Upsilon}+\beta^{*} \cos \Theta \sinh \eta_{\Upsilon}\right]\right\}
$$

and its pdf, by definition

$$
f_{B}(\eta)=\int_{-1}^{1} d \cos \Theta p_{\Omega}(\cos \Theta) \delta[\eta-\eta(\cos \Theta)] .
$$

Under a change of variables $\zeta=\eta(\cos \Theta)$, one finds

$$
\begin{aligned}
f_{B}(\eta) & =\frac{3}{4 \beta^{*} \cosh \eta^{*}} \int_{\left|\eta_{\Upsilon} \eta^{*}\right|}^{\eta_{\Upsilon}+\eta^{*}} d \zeta \frac{\delta[\eta-\zeta] \sinh \zeta}{\sinh \eta_{\Upsilon}}\left[1-\left(\frac{\cosh \zeta-\cosh \eta^{*} \cosh \eta_{\Upsilon}}{\sinh \eta^{*} \sinh \eta_{\Upsilon}}\right)^{2}\right] \\
& =\frac{3 \sinh \eta}{4 \sinh \eta^{*} \sinh \eta_{\Upsilon}}\left[1-\left(\frac{\cosh \eta-\cosh \eta^{*} \cosh \eta_{\Upsilon}}{\sinh \eta^{*} \sinh \eta_{\Upsilon}}\right)^{2}\right], \quad\left|\eta_{\Upsilon}-\eta^{*}\right|<\eta<\eta^{*}+\eta_{\Upsilon},
\end{aligned}
$$

and zero otherwise. Note that $f_{B}$ itself has zeroes at each end of its non-trivial domain, i.e. at $\eta=\eta^{*}+\eta_{\Upsilon}$ and $\left|\eta_{\Upsilon}-\eta^{*}\right|$. The boost at the pdf peak is $\gamma_{\text {peak }}=\left(\mathcal{E}_{+}+\mathcal{E}_{-}\right) /(2 m)$. E.g.

for the Belle II parameters, the peak $\beta \gamma_{\text {peak }}=\sinh \cosh ^{-1}\left(\gamma_{\text {peak }}\right)=0.29$. This is the boost of $B^{\prime}$ s emitted at $\Theta=\pi / 2$, and matches the quoted $B$ design boost at Belle II [302].

\section{D.III The Monte Carlo event generator}

This appendix describes in more detail the MC event generator written in $\mathrm{C}$ and Python. The $B \rightarrow\left(K^{*} \rightarrow K \pi\right) \gamma$ phase space is generated as follows. The $B$ rapidity is sampled from the PDF given in eq. (D.10) while the photon polar angle $\theta_{\gamma}$ and the $K$ polar and 

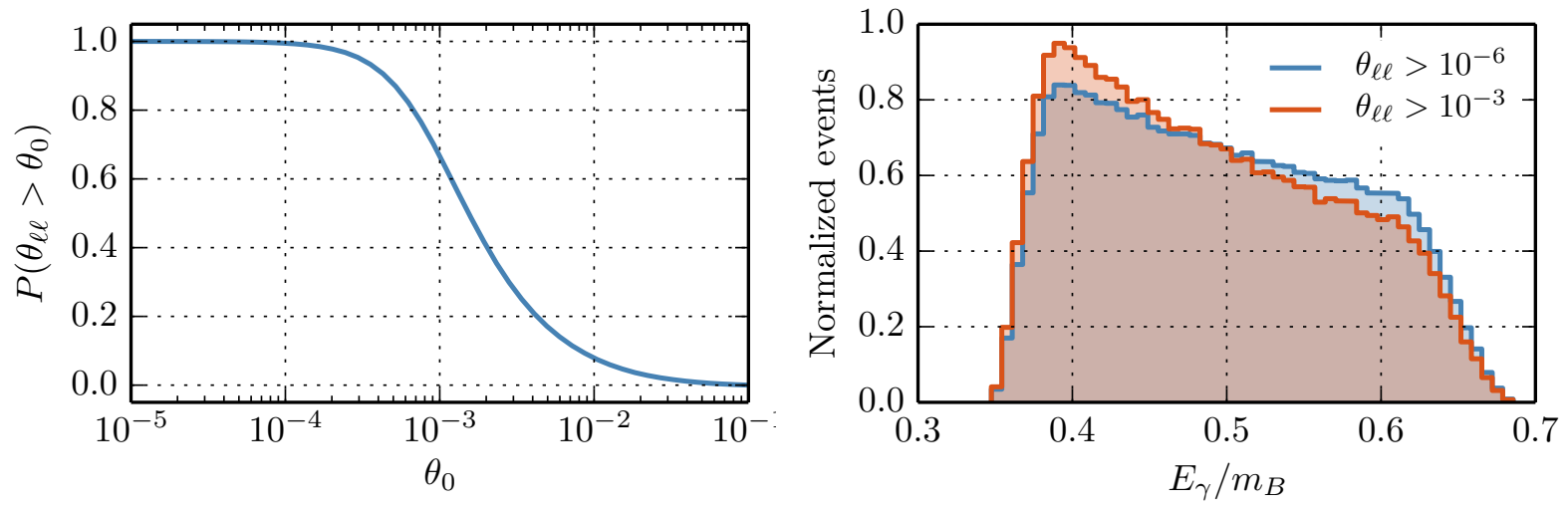

Figure D.1: Left: The cumulative distribution function (CDF) of the opening angle between the leptons. Right: The normalized distribution of the photon energy in units of the $B$ mass for two different $\theta_{\ell \ell}$ cuts.

azimuthal angles are generated uniformly in the appropriate frame. On the other hand, since in BH photon conversion the leptons are produced with preferentially small angles with respect to the photon direction, the lepton polar angles are generated uniformly on a log scale. This is implemented via the transformed variables $t_{ \pm}=\log _{10} \theta_{ \pm}$ where $t_{ \pm}$are uniformly distributed and with $t_{ \pm} \in[-5,-1]$. Moreover, the azimuthal angle separation between the leptons $(\delta \phi)$ is peaked around $\pi$ and so, to improve the efficiency of the generator, $\delta \phi$ is sampled from a Cauchy distribution. All other $\mathrm{BH}$ variables are generated uniformly.

The weight associated with each event is proportional to the matrix element (D.4). The events are unweighted using the standard procedure. That is, the weights are normalized to the largest weight and the event is kept if its normalized weight is larger than a random number on $[0,1]$. Of course, this procedure assumes that the phase space was sufficiently sampled such that the largest weight found is close to the global maximum.

Using this procedure, we generate MC samples for many choices of $(r, \phi+\delta)$ couplets with 500k events per sample. Some representative distributions from the $(0.1,0)$ sample are shown in Figs D.1 and D.2. In particular, the left panel in Fig. D.1 shows the 

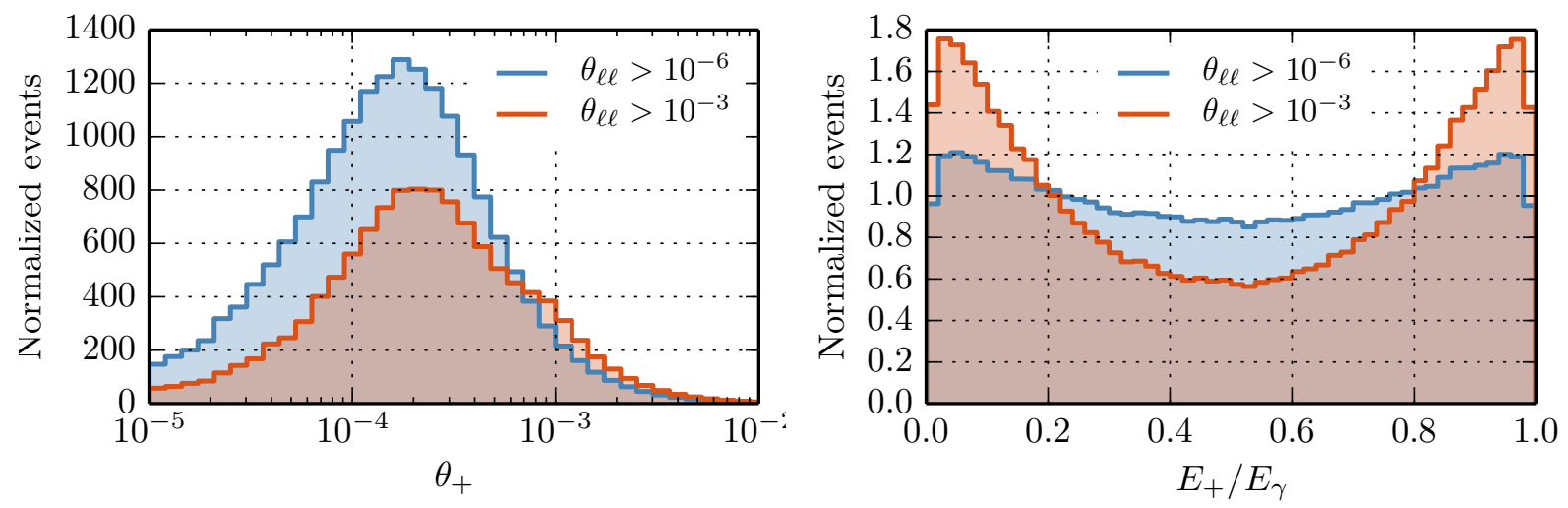

Figure D.2: Left: The normalized polar angle distribution of the positron for two different values of the opening angle cut $\theta_{\ell \ell}$. Right: the positron energy as a fraction of the photon energy for two values of $\theta_{\ell \ell}$. The distribution exhibits the expected behavior for $\mathrm{BH}$ conversion. It is symmetric about $1 / 2$ and prefers that one lepton carry a larger fraction of the photon energy.

cumulative distribution function for the opening angle between the leptons $\theta_{\ell \ell}$ while the right panel shows the distribution of photons energies. Figure D. 2 shows the polar angle and fractional energy distribution of the positron. 


\section{Bibliography}

[1] H. M. Georgi, Lie algebras in particle physics; 2nd ed. Frontiers in Physics. Perseus, Cambridge, 1999.

[2] B. Batell, J. Pradler, and M. Spannowsky, Dark Matter from Minimal Flavor Violation, JHEP 1108 (2011) 038, [arXiv: 1105.1781].

[3] A. Sakharov, Violation of CP Invariance, c Asymmetry, and Baryon Asymmetry of the Universe, Pisma Zh.Eksp.Teor.Fiz. 5 (1967) 32-35.

[4] T. Hambye, On the stability of particle dark matter, PoS IDM2010 (2011) 098, [arXiv: 1012.4587].

[5] L. Ackerman, M. R. Buckley, S. M. Carroll, and M. Kamionkowski, Dark Matter and Dark Radiation, Phys.Rev. D79 (2009) 023519, [arXiv: 0810 .5126].

[6] J. L. Feng, H. Tu, and H.-B. Yu, Thermal Relics in Hidden Sectors, JCAP 0810 (2008) 043, [arXiv: 0808.2318].

[7] J. L. Feng, M. Kaplinghat, H. Tu, and H.-B. Yu, Hidden Charged Dark Matter, JCAP 0907 (2009) 004, [arXiv: 0905.3039$].$

[8] G. R. Farrar and P. Fayet, Phenomenology of the Production, Decay, and Detection of New Hadronic States Associated with Supersymmetry, Phys.Lett. B76 (1978) 575-579. 
[9] S. Dimopoulos, S. Raby, and F. Wilczek, Proton Decay in Supersymmetric Models, Phys.Lett. B112 (1982) 133.

[10] G. R. Farrar and S. Weinberg, Supersymmetry at Ordinary Energies. 2. R Invariance, Goldstone Bosons, and Gauge Fermion Masses, Phys.Rev. D27 (1983) 2732.

[11] M. Kadastik, K. Kannike, and M. Raidal, Matter parity as the origin of scalar Dark Matter, Phys.Rev. D81 (2010) 015002, [arXiv:0903.2475].

[12] M. Kadastik, K. Kannike, and M. Raidal, Dark Matter as the signal of Grand Unification, Phys.Rev. D80 (2009) 085020, [arXiv: 0907.1894].

[13] M. Frigerio and T. Hambye, Dark matter stability and unification without supersymmetry, Phys.Rev. D81 (2010) 075002, [arXiv:0912.1545].

[14] M. Cirelli and A. Strumia, Minimal Dark Matter: Model and results, New J.Phys. 11 (2009) 105005, [arXiv: 0903.3381].

[15] M. Cirelli, N. Fornengo, and A. Strumia, Minimal dark matter, Nucl.Phys. B753 (2006) 178-194, [hep-ph/0512090].

[16] T. Hambye, Hidden vector dark matter, JHEP 0901 (2009) 028, [arXiv:0811.0172].

[17] Y. Bai and R. J. Hill, Weakly Interacting Stable Pions, Phys.Rev. D82 (2010) 111701, [arXiv: 1005.0008].

[18] D. E. Kaplan, M. A. Luty, and K. M. Zurek, Asymmetric Dark Matter, Phys.Rev. D79 (2009) 115016, [arXiv: 0901.4117$].$

[19] K. M. Zurek, Asymmetric Dark Matter: Theories, Signatures, and Constraints, Phys.Rept. 537 (2014) 91-121, [arXiv: 1308 .0338].

[20] K. Petraki and R. R. Volkas, Review of asymmetric dark matter, Int.J.Mod.Phys. A28 (2013) 1330028, [arXiv: 1305.4939]. 
[21] J. Kile and A. Soni, Flavored Dark Matter in Direct Detection Experiments and at LHC, Phys.Rev. D84 (2011) 035016, [arXiv: 1104.5239].

[22] H. Davoudiasl, D. E. Morrissey, K. Sigurdson, and S. Tulin, Baryon Destruction by Asymmetric Dark Matter, Phys.Rev. D84 (2011) 096008, [arXiv: 1106 .4320].

[23] L. Lopez-Honorez and L. Merlo, Dark matter within the minimal flavour violation ansatz, Phys.Lett. B722 (2013) 135-143, [arXiv: 1303.1087].

[24] P. Agrawal, M. Blanke, and K. Gemmler, Flavored dark matter beyond Minimal Flavor Violation, JHEP 1410 (2014) 72, [arXiv : 1405. 6709].

[25] B. Batell, T. Lin, and L.-T. Wang, Flavored Dark Matter and R-Parity Violation, JHEP 1401 (2014) 075, [arXiv: 1309.4462].

[26] P. Agrawal, B. Batell, D. Hooper, and T. Lin, Flavored Dark Matter and the Galactic Center Gamma-Ray Excess, Phys.Rev. D90 (2014), no. 6 063512, [arXiv: 1404.1373].

[27] U. Haisch, A. Hibbs, and E. Re, Determining the structure of dark-matter couplings at the LHC, Phys.Rev. D89 (2014), no. 3 034009, [arXiv: 1311.7131].

[28] J. F. Kamenik and J. Zupan, Discovering Dark Matter Through Flavor Violation at the LHC, Phys.Rev. D84 (2011) 111502, [arXiv:1107.0623].

[29] P. Agrawal, S. Blanchet, Z. Chacko, and C. Kilic, Flavored Dark Matter, and Its Implications for Direct Detection and Colliders, Phys.Rev. D86 (2012) 055002, [arXiv:1109.3516].

[30] A. Merle and V. Niro, Deriving Models for keV sterile Neutrino Dark Matter with the Froggatt-Nielsen mechanism, JCAP 1107 (2011) 023, [arXiv: 1105. 5136]. 
[31] Y. Kajiyama, K. Kannike, and M. Raidal, Symmetryless Dark Matter, Phys.Rev. D85 (2012) 033008, [arXiv: 1111.1270].

[32] M. Lattanzi, R. A. Lineros, and M. Taoso, Connecting neutrino physics with dark matter, arXiv:1406.0004.

[33] M. Hirsch, S. Morisi, E. Peinado, and J. Valle, Discrete dark matter, Phys.Rev. D82 (2010) 116003, [arXiv:1007.0871].

[34] M. Boucenna, S. Morisi, E. Peinado, Y. Shimizu, and J. Valle, Predictive discrete dark matter model and neutrino oscillations, Phys.Rev. D86 (2012) 073008, [arXiv: 1204.4733].

[35] H. An, S.-L. Chen, R. N. Mohapatra, and Y. Zhang, Leptogenesis as a Common Origin for Matter and Dark Matter, JHEP 1003 (2010) 124, [arXiv : 0911.4463].

[36] K. Agashe and G. Servant, Warped unification, proton stability and dark matter, Phys.Rev.Lett. 93 (2004) 231805, [hep-ph/0403143].

[37] Y. Zhao and K. M. Zurek, Indirect Detection Signatures for the Origin of Asymmetric Dark Matter, JHEP 1407 (2014) 017, [arXiv: 1401.7664].

[38] L. Feng and Z. Kang, Decaying Asymmetric Dark Matter Relaxes the AMS-Fermi Tension, JCAP 1310 (2013) 008, [arXiv: 1304 .7492].

[39] I. Masina, P. Panci, and F. Sannino, Gamma Ray Constraints on Flavor Violating Asymmetric Dark Matter, JCAP 1212 (2012) 002, [arXiv: 1205. 5918].

[40] I. Masina and F. Sannino, Charge Asymmetric Cosmic Rays as a probe of Flavor Violating Asymmetric Dark Matter, JCAP 1109 (2011) 021, [arXiv: 1106 . 3353].

[41] S. Nussinov, Technocosmology — could a technibaryon excess provide a "natural" missing mass candidate?, Physics Letters B 165 (1985), no. 1-3 55 - 58. 
[42] S. M. Barr, R. S. Chivukula, and E. Farhi, Electroweak Fermion Number Violation and the Production of Stable Particles in the Early Universe, Phys.Lett. B241 (1990) 387-391.

[43] D. B. Kaplan, A Single explanation for both the baryon and dark matter densities, Phys.Rev.Lett. 68 (1992) 741-743.

[44] S. M. Barr, Baryogenesis, sphalerons and the cogeneration of dark matter, Phys.Rev. D44 (1991) 3062-3066.

[45] S. Dodelson, B. R. Greene, and L. M. Widrow, Baryogenesis, dark matter and the width of the Z, Nucl.Phys. B372 (1992) 467-493.

[46] V. A. Kuzmin, A Simultaneous solution to baryogenesis and dark matter problems, Phys.Part.Nucl. 29 (1998) 257-265, [hep-ph/9701269].

[47] M. Fujii and T. Yanagida, A Solution to the coincidence puzzle of Omega(B) and Omega (DM), Phys.Lett. B542 (2002) 80-88, [hep-ph/0206066].

[48] D. Hooper, J. March-Russell, and S. M. West, Asymmetric sneutrino dark matter and the Omega(b) / Omega(DM) puzzle, Phys.Lett. B605 (2005) 228-236, [hep-ph/0410114].

[49] R. Kitano and I. Low, Dark matter from baryon asymmetry, Phys.Rev. D71 (2005) 023510, [hep-ph/0411133].

[50] S. B. Gudnason, C. Kouvaris, and F. Sannino, Towards working technicolor: Effective theories and dark matter, Phys.Rev. D73 (2006) 115003, [hep-ph/0603014].

[51] R. Kitano, H. Murayama, and M. Ratz, Unified origin of baryons and dark matter, Phys.Lett. B669 (2008) 145-149, [arXiv: 0807.4313]. 
[52] L. J. Hall, J. March-Russell, and S. M. West, A Unified Theory of Matter Genesis: Asymmetric Freeze-In, arXiv:1010.0245.

[53] J. March-Russell and M. McCullough, Asymmetric Dark Matter via Spontaneous Co-Genesis, JCAP 1203 (2012) 019, [arXiv: 1106 . 4319].

[54] M. L. Graesser, I. M. Shoemaker, and L. Vecchi, Asymmetric WIMP dark matter, JHEP 1110 (2011) 110, [arXiv:1103.2771].

[55] Particle Data Group Collaboration, K. Olive et al., Review of Particle Physics, Chin.Phys. C38 (2014) 090001.

[56] Planck Collaboration, P. Ade et al., Planck 2013 results. XVI. Cosmological parameters, arXiv:1303.5076.

[57] A. Falkowski, J. T. Ruderman, and T. Volansky, Asymmetric Dark Matter from Leptogenesis, JHEP 1105 (2011) 106, [arXiv:1101.4936].

[58] Y. Cui, L. Randall, and B. Shuve, Emergent Dark Matter, Baryon, and Lepton Numbers, JHEP 1108 (2011) 073, [arXiv: 1106 .4834].

[59] T. Lin, H.-B. Yu, and K. M. Zurek, On Symmetric and Asymmetric Light Dark Matter, Phys.Rev. D85 (2012) 063503, [arXiv:1111 .0293].

[60] T. Cohen, D. J. Phalen, A. Pierce, and K. M. Zurek, Asymmetric Dark Matter from a GeV Hidden Sector, Phys.Rev. D82 (2010) 056001, [arXiv: 1005.1655].

[61] M. Blennow, E. Fernandez-Martinez, O. Mena, J. Redondo, and P. Serra, Asymmetric Dark Matter and Dark Radiation, JCAP 1207 (2012) 022, [arXiv: 1203.5803]. 
[62] J. March-Russell, J. Unwin, and S. M. West, Closing in on Asymmetric Dark Matter I: Model independent limits for interactions with quarks, JHEP 1208 (2012) 029, [arXiv: 1203.4854].

[63] R. S. Chivukula and H. Georgi, Composite Technicolor Standard Model, Phys.Lett. B188 (1987) 99.

[64] G. D'Ambrosio, G. Giudice, G. Isidori, and A. Strumia, Minimal flavor violation: An Effective field theory approach, Nucl.Phys. B645 (2002) 155-187, [hep-ph/0207036].

[65] L. Hall and L. Randall, Weak scale effective supersymmetry, Phys.Rev.Lett. 65 (1990) 2939-2942.

[66] A. J. Buras, Minimal flavor violation, Acta Phys.Polon. B34 (2003) 5615-5668, [hep-ph/0310208].

[67] A. Buras, P. Gambino, M. Gorbahn, S. Jager, and L. Silvestrini, Universal unitarity triangle and physics beyond the standard model, Phys.Lett. B500 (2001) 161-167, [hep-ph/0007085].

[68] C. Froggatt and H. B. Nielsen, Hierarchy of Quark Masses, Cabibbo Angles and CP Violation, Nucl.Phys. B147 (1979) 277.

[69] M. Leurer, Y. Nir, and N. Seiberg, Mass matrix models: The Sequel, Nucl.Phys. B420 (1994) 468-504, [hep-ph/9310320].

[70] LAT Collaboration, M. Ackermann et al., Constraints on the Galactic Halo Dark Matter from Fermi-LAT Diffuse Measurements, Astrophys.J. 761 (2012) 91, [arXiv: 1205.6474]. 
[71] AMS Collaboration, M. Aguilar et al., First Result from the Alpha Magnetic Spectrometer on the International Space Station: Precision Measurement of the Positron Fraction in Primary Cosmic Rays of 0.5-350 GeV, Phys.Rev.Lett. 110 (2013), no. 14141102.

[72] A. Ibarra, A. S. Lamperstorfer, and J. Silk, Dark matter annihilations and decays after the AMS-02 positron measurements, arXiv:1309.2570.

[73] Super-Kamiokande Collaboration, S. Desai et al., Search for dark matter WIMPs using upward through-going muons in Super-Kamiokande, Phys.Rev. D70 (2004) 083523, [hep-ex/0404025].

[74] L. Covi, M. Grefe, A. Ibarra, and D. Tran, Neutrino Signals from Dark Matter Decay, JCAP 1004 (2010) 017, [arXiv: 0912.3521].

[75] Fermi-LAT Collaboration, M. Ackermann, M. Ajello, W. Atwood, L. Baldini, J. Ballet, G. Barbiellini, D. Bastieri, K. Bechtol, R. Bellazzini, B. Berenji, et al., Fermi-LAT Observations of the Diffuse Gamma-Ray Emission: Implications for Cosmic Rays and the Interstellar Medium, Astrophys.J. 750 (2012) 3, [arXiv: 1202.4039].

[76] LAT Collaboration, M. Ackermann et al., Fermi LAT Search for Dark Matter in Gamma-ray Lines and the Inclusive Photon Spectrum, Phys.Rev. D86 (2012) 022002, [arXiv: 1205.2739].

[77] Fermi-LAT Collaboration, A. Abdo et al., The Spectrum of the Isotropic Diffuse Gamma-Ray Emission Derived From First-Year Fermi Large Area Telescope Data, Phys.Rev.Lett. 104 (2010) 101101, [arXiv: 1002.3603].

[78] T. Han, I. Lewis, and T. McElmurry, QCD Corrections to Scalar Diquark Production at Hadron Colliders, JHEP 1001 (2010) 123, [arXiv: 0909. 2666]. 
[79] B. Bhattacherjee, S. Matsumoto, S. Mukhopadhyay, and M. M. Nojiri, Phenomenology of light fermionic asymmetric dark matter, JHEP 1310 (2013) 032, [arXiv: 1306.5878].

[80] G. F. Giudice, B. Gripaios, and R. Sundrum, Flavourful Production at Hadron Colliders, JHEP 1108 (2011) 055, [arXiv : 1105.3161].

[81] B. Grinstein, A. L. Kagan, J. Zupan, and M. Trott, Flavor Symmetric Sectors and Collider Physics, JHEP 1110 (2011) 072, [arXiv : 1108 . 4027].

[82] UTfit Collaboration, M. Bona et al., Model-independent constraints on $\Delta F=2$ operators and the scale of new physics, JHEP 0803 (2008) 049, [arXiv: 0707 . 0636].

[83] J. Charles, S. Descotes-Genon, Z. Ligeti, S. Monteil, M. Papucci, et al., Future sensitivity to new physics in $B_{d}, B_{s}$ and K mixings, Phys.Rev. D89 (2014) 033016, [arXiv: 1309.2293].

[84] CMS Collaboration, S. Chatrchyan et al., Search for pair-produced dijet resonances in four-jet final states in pp collisions at $\sqrt{s}=7 \mathrm{TeV}$, Phys.Rev.Lett. 110 (2013) 141802, [arXiv: 1302.0531].

[85] CMS Collaboration, Search for direct production of bottom squark pairs, Tech. Rep. CMS-PAS-SUS-13-018, CERN, Geneva, 2014.

[86] ATLAS and CMS Collaboration, Combination of single top-quark cross-sections measurements in the t-channel at $\sqrt{s}=8 \mathrm{TeV}$ with the ATLAS and CMS experiments, Tech. Rep. CMS-PAS-TOP-12-002, ATLAS-COM-CONF-2013-061, ATLAS-CONF-2013-098, 2013.

[87] G. Jungman, M. Kamionkowski, and K. Griest, Supersymmetric dark matter, Phys.Rept. 267 (1996) 195-373, [hep-ph/9506380]. 
[88] M. Boucenna, M. Hirsch, S. Morisi, E. Peinado, M. Taoso, et al., Phenomenology of Dark Matter from $A_{4}$ Flavor Symmetry, JHEP 1105 (2011) 037, [arXiv:1101.2874].

[89] D. Meloni, S. Morisi, and E. Peinado, Neutrino phenomenology and stable dark matter with A4, Phys.Lett. B697 (2011) 339-342, [arXiv : 1011.1371].

[90] M. Lindner, D. Schmidt, and T. Schwetz, Dark Matter and Neutrino Masses from Global $U(1)_{B-L}$ Symmetry Breaking, Phys.Lett. B705 (2011) 324-330, [arXiv: 1105.4626].

[91] B. Grinstein, M. Redi, and G. Villadoro, Low Scale Flavor Gauge Symmetries, JHEP 1011 (2010) 067, [arXiv: 1009.2049].

[92] C.-J. Lee and J. Tandean, Lepton-Flavored Scalar Dark Matter with Minimal Flavor Violation, arXiv:1410.6803.

[93] F. Bishara and J. Zupan, Continuous Flavor Symmetries and the Stability of Asymmetric Dark Matter, JHEP 1501 (2015) 089, [arXiv: 1408. 3852].

[94] A. Hamze, C. Kilic, J. Koeller, C. Trendafilova, and J.-H. Yu, Lepton-Flavored Asymmetric Dark Matter and Interference in Direct Detection, Phys.Rev. D91 (2015), no. 3 035009, [arXiv: 1410.3030].

[95] A. Kumar and S. Tulin, Top-flavored dark matter and the forward-backward asymmetry, Phys.Rev. D87 (2013), no. 9 095006, [arXiv: 1303.0332].

[96] J. Kile, A. Kobach, and A. Soni, Lepton-Flavored Dark Matter, arXiv:1411.1407.

[97] L. Calibbi, A. Crivellin, and B. Zaldivar, The Flavour Portal to Dark Matter, arXiv:1501.0726. 
[98] E. Gabrielli and G. Giudice, Supersymmetric corrections to epsilon prime / epsilon at the leading order in QCD and QED, Nucl.Phys. B433 (1995) 3-25, [hep-lat/9407029].

[99] A. Ali and D. London, Profiles of the unitarity triangle and CP violating phases in the standard model and supersymmetric theories, Eur.Phys.J. C9 (1999) 687-703, [hep-ph/9903535].

[100] A. L. Kagan, G. Perez, T. Volansky, and J. Zupan, General Minimal Flavor Violation, Phys.Rev. D80 (2009) 076002, [arXiv: 0903.1794].

[101] C. Smith, Proton stability from a fourth family, Phys.Rev. D85 (2012) 036005, [arXiv: 1105.1723].

[102] A. J. Buras, M. V. Carlucci, L. Merlo, and E. Stamou, Phenomenology of a Gauged SU $(3)^{3}$ Flavour Model, JHEP 1203 (2012) 088, [arXiv: 1112.4477].

[103] P. Gondolo and G. Gelmini, Cosmic abundances of stable particles: Improved analysis, Nucl.Phys. B360 (1991) 145-179.

[104] K. Griest and D. Seckel, Three exceptions in the calculation of relic abundances, Phys.Rev. D43 (1991) 3191-3203.

[105] M. Backovic, K. Kong, and M. McCaskey, MadDM v.1.0: Computation of Dark Matter Relic Abundance Using MadGraph5, Physics of the Dark Universe 5-6 (2014) 18-28, [arXiv:1308.4955].

[106] A. Alloul, N. D. Christensen, C. Degrande, C. Duhr, and B. Fuks, FeynRules 2.0 A complete toolbox for tree-level phenomenology, Comput.Phys.Commun. 185 (2014) 2250-2300, [arXiv:1310 .1921].

[107] B. Fields and S. Sarkar, Big-Bang nucleosynthesis (2006 Particle Data Group mini-review), astro-ph/0601514. 
[108] W. Hu and J. Silk, Thermalization and spectral distortions of the cosmic background radiation, Phys.Rev. D48 (1993) 485-502.

[109] W. Hu and J. Silk, Thermalization constraints and spectral distortions for massive unstable relic particles, Phys.Rev.Lett. 70 (1993) 2661-2664.

[110] R. Essig, E. Kuflik, S. D. McDermott, T. Volansky, and K. M. Zurek, Constraining Light Dark Matter with Diffuse X-Ray and Gamma-Ray Observations, JHEP 1311 (2013) 193, [arXiv: 1309.4091].

[111] F. Iocco, G. Mangano, G. Miele, O. Pisanti, and P. D. Serpico, Primordial Nucleosynthesis: from precision cosmology to fundamental physics, Phys.Rept. $\mathbf{4 7 2}$ (2009) 1-76, [arXiv: 0809.0631].

[112] D. Lindley, Cosmological Constraints on the Lifetime of Massive Particles, Astrophys.J. 294 (1985) 1-8.

[113] M. Reno and D. Seckel, Primordial Nucleosynthesis: The Effects of Injecting Hadrons, Phys.Rev. D37 (1988) 3441.

[114] S. Dimopoulos, R. Esmailzadeh, L. J. Hall, and G. Starkman, Is the Universe Closed by Baryons? Nucleosynthesis With a Late Decaying Massive Particle, Astrophys.J. 330 (1988) 545.

[115] R. J. Scherrer and M. S. Turner, Primordial Nucleosynthesis with Decaying Particles. 1. Entropy Producing Decays. 2. Inert Decays, Astrophys.J. 331 (1988) 19-32.

[116] J. R. Ellis, G. Gelmini, J. L. Lopez, D. V. Nanopoulos, and S. Sarkar, Astrophysical constraints on massive unstable neutral relic particles, Nucl.Phys. B373 (1992) 399-437.

[117] M. Kawasaki, K. Kohri, and T. Moroi, Big-Bang nucleosynthesis and hadronic decay of long-lived massive particles, Phys.Rev. D71 (2005) 083502, [astro-ph/0408426]. 
[118] G. Belanger, F. Boudjema, A. Pukhov, and A. Semenov, Dark matter direct detection rate in a generic model with micrOMEGAs 2.2, Comput.Phys.Commun. 180 (2009) 747-767, [arXiv: 0803.2360].

[119] G. Arcadi, Y. Mambrini, M. H. G. Tytgat, and B. Zaldivar, Invisible Z' and dark matter: LHC vs LUX constraints, JHEP 1403 (2014) 134, [arXiv: 1401.0221].

[120] LUX Collaboration, D. Akerib et al., First results from the LUX dark matter experiment at the Sanford Underground Research Facility, Phys.Rev.Lett. 112 (2014), no. 9 091303, [arXiv: 1310.8214].

[121] A. Urbano and W. Xue, Constraining the Higgs portal with antiprotons, arXiv:1412.3798.

[122] J. M. Cline and K. Kainulainen, Electroweak baryogenesis and dark matter from a singlet Higgs, JCAP 1301 (2013) 012, [arXiv: 1210 .4196].

[123] P. Junnarkar and A. Walker-Loud, Scalar strange content of the nucleon from lattice QCD, Phys.Rev. D87 (2013), no. 11 114510, [arXiv:1301.1114].

[124] J. Alarcon, J. Martin Camalich, and J. Oller, The chiral representation of the $\pi N$ scattering amplitude and the pion-nucleon sigma term, Phys.Rev. D85 (2012) 051503, [arXiv:1110.3797].

[125] L. Alvarez-Ruso, T. Ledwig, J. Martin Camalich, and M. Vicente Vacas, Nucleon mass and pion-nucleon sigma term from a chiral analysis of lattice QCD world data, EPJ Web Conf. 73 (2014) 04015.

[126] A. Crivellin, F. D’Eramo, and M. Procura, New Constraints on Dark Matter Effective Theories from Standard Model Loops, Phys.Rev.Lett. 112 (2014) 191304, [arXiv: 1402.1173]. 
[127] A. Crivellin, M. Hoferichter, and M. Procura, Accurate evaluation of hadronic uncertainties in spin-independent WIMP-nucleon scattering: Disentangling two- and three-flavor effects, Phys.Rev. D89 (2014), no. 5 054021, [arXiv: 1312.4951].

[128] M. Boudaud, M. Cirelli, G. Giesen, and P. Salati, A fussy revisitation of antiprotons as a tool for Dark Matter searches, arXiv:1412.5696.

[129] O. Adriani, G. Bazilevskaya, G. Barbarino, R. Bellotti, M. Boezio, et al., Measurement of the flux of primary cosmic ray antiprotons with energies of $60-\mathrm{MeV}$ to 350-GeV in the PAMELA experiment, JETP Lett. 96 (2013) 621-627.

[130] Fermi-LAT Collaboration, M. Ackermann et al., Searching for Dark Matter Annihilation from Milky Way Dwarf Spheroidal Galaxies with Six Years of Fermi-LAT Data, arXiv: 1503.0264.

[131] M. R. Buckley, E. Charles, J. M. Gaskins, A. M. Brooks, A. Drlica-Wagner, et al., Search for Gamma-ray Emission from Dark Matter Annihilation in the Large Magellanic Cloud with the Fermi Large Area Telescope, Phys.Rev.D (2015) [arXiv: 1502.0102].

[132] Fermi-LAT Collaboration, M. Ackermann et al., Limits on Dark Matter Annihilation Signals from the Fermi LAT 4-year Measurement of the Isotropic Gamma-Ray Background, arXiv: 1501.0546.

[133] ATLAS Collaboration, G. Aad et al., Search for new phenomena in the dijet mass distribution using pp collision data at $\sqrt{s}=8 \mathrm{TeV}$ with the ATLAS detector, arXiv:1407.1376.

[134] CMS Collaboration, S. Chatrchyan et al., Inclusive search for a vector-like T quark with charge $\frac{2}{3}$ in pp collisions at $\sqrt{s}=8 \mathrm{TeV}$, Phys.Lett. B729 (2014) 149-171, [arXiv: 1311.7667]. 
[135] E. Eichten, I. Hinchliffe, K. D. Lane, and C. Quigg, Super Collider Physics, Rev.Mod.Phys. 56 (1984) 579-707.

[136] K. D. Lane and M. Ramana, Walking technicolor signatures at hadron colliders, Phys.Rev. D44 (1991) 2678-2700.

[137] A. J. Buras, S. Jager, and J. Urban, Master formulae for Delta F=2 NLO QCD factors in the standard model and beyond, Nucl.Phys. B605 (2001) 600-624, [hep-ph/0102316].

[138] J. Laiho, E. Lunghi, and R. S. Van de Water, Lattice QCD inputs to the CKM unitarity triangle analysis, Phys.Rev. D81 (2010) 034503, [arXiv: 0910 . 2928].

[139] A. J. Buras, D. Guadagnoli, and G. Isidori, On $\epsilon_{K}$ beyond lowest order in the Operator Product Expansion, Phys.Lett. B688 (2010) 309-313, [arXiv: 1002 . 3612].

[140] A. J. Buras and D. Guadagnoli, Correlations among new CP violating effects in $\Delta F$ $=2$ observables, Phys.Rev. D78 (2008) 033005, [arXiv:0805.3887].

[141] J. Brod and M. Gorbahn, Next-to-Next-to-Leading-Order Charm-Quark Contribution to the CP Violation Parameter $\epsilon_{K}$ and $\Delta M_{K}$, Phys.Rev.Lett. 108 (2012) 121801, [arXiv: 1108.2036].

[142] J. Brod and M. Gorbahn, $\epsilon_{K}$ at Next-to-Next-to-Leading Order: The Charm-Top-Quark Contribution, Phys.Rev. D82 (2010) 094026, [arXiv: 1007 .0684].

[143] A. J. Buras, L. Merlo, and E. Stamou, The Impact of Flavour Changing Neutral Gauge Bosons on $\bar{B} \rightarrow X_{s} \gamma$, JHEP 1108 (2011) 124, [arXiv: 1105.5146].

[144] M. Misiak, H. Asatrian, K. Bieri, M. Czakon, A. Czarnecki, et al., Estimate of B(anti-B —Egt; X(s) gamma) at O(alpha(s)**2), Phys.Rev.Lett. 98 (2007) 022002, [hep-ph/0609232]. 
[145] P. Gambino and M. Misiak, Quark mass effects in anti-B —Egt; X(s gamma), Nucl.Phys. B611 (2001) 338-366, [hep-ph/0104034].

[146] M. Misiak and M. Steinhauser, NNLO QCD corrections to the anti-B - Egt; X(s) gamma matrix elements using interpolation in m(c), Nucl.Phys. B764 (2007) 62-82, [hep-ph/0609241].

[147] B. Patt and F. Wilczek, Higgs-field portal into hidden sectors, hep-ph/0605188.

[148] J. March-Russell, S. M. West, D. Cumberbatch, and D. Hooper, Heavy Dark Matter Through the Higgs Portal, JHEP 0807 (2008) 058, [arXiv: 0801 . 3440].

[149] S. Andreas, T. Hambye, and M. H. Tytgat, WIMP dark matter, Higgs exchange and DAMA, JCAP 0810 (2008) 034, [arXiv: 0808.0255].

[150] C. Englert, T. Plehn, D. Zerwas, and P. M. Zerwas, Exploring the Higgs portal, Phys.Lett. B703 (2011) 298-305, [arXiv: 1106 . 3097].

[151] O. Lebedev, H. M. Lee, and Y. Mambrini, Vector Higgs-portal dark matter and the invisible Higgs, Phys.Lett. B707 (2012) 570-576, [arXiv:1111 .4482].

[152] L. Lopez-Honorez, T. Schwetz, and J. Zupan, Higgs portal, fermionic dark matter, and a Standard Model like Higgs at 125 GeV, Phys.Lett. B716 (2012) 179-185, [arXiv: 1203.2064].

[153] A. Djouadi, A. Falkowski, Y. Mambrini, and J. Quevillon, Direct Detection of Higgs-Portal Dark Matter at the LHC, Eur.Phys.J. C73 (2013), no. 6 2455, [arXiv:1205.3169].

[154] A. Greljo, J. Julio, J. F. Kamenik, C. Smith, and J. Zupan, Constraining Higgs mediated dark matter interactions, JHEP 1311 (2013) 190, [arXiv: 1309. 3561]. 
[155] M. A. Fedderke, J.-Y. Chen, E. W. Kolb, and L.-T. Wang, The Fermionic Dark Matter Higgs Portal: an effective field theory approach, JHEP 1408 (2014) 122, [arXiv: 1404.2283].

[156] N. Craig, H. K. Lou, M. McCullough, and A. Thalapillil, The Higgs Portal Above Threshold, arXiv:1412.0258.

[157] ATLAS Collaboration, Combined coupling measurements of the Higgs-like boson with the ATLAS detector using up to $25 \mathrm{fb}^{-1}$ of proton-proton collision data, Tech. Rep. ATLAS-CONF-2013-034, CERN, Geneva, Mar, 2013.

[158] CMS Collaboration, Combination of standard model Higgs boson searches and measurements of the properties of the new boson with a mass near $125 \mathrm{GeV}$, Tech. Rep. CMS-PAS-HIG-13-005, CERN, Geneva, 2013.

[159] A. L. Kagan, G. Perez, F. Petriello, Y. Soreq, S. Stoynev, et al., An Exclusive Window onto Higgs Yukawa Couplings, arXiv: 1406.1722.

[160] G. Perez, Y. Soreq, E. Stamou, and K. Tobioka, Constraining the Charm Yukawa and Higgs-quark Universality, arXiv:1503.0029.

[161] C. Delaunay, T. Golling, G. Perez, and Y. Soreq, Enhanced Higgs boson coupling to charm pairs, Phys.Rev. D89 (2014), no. 3 033014, [arXiv: 1310.7029].

[162] A. Efrati, A. Falkowski, and Y. Soreq, Electroweak constraints on flavorful effective theories, arXiv: 1503.0787.

[163] R. A. Porto and A. Zee, The Private Higgs, Phys.Lett. B666 (2008) 491-495, [arXiv: 0712.0448].

[164] C. Kilic, M. D. Klimek, and J.-H. Yu, Signatures of Top Flavored Dark Matter, arXiv: 1501.0220. 
[165] P. Agrawal, Z. Chacko, C. Kilic, and C. B. Verhaaren, A Couplet from Flavored Dark Matter, arXiv:1503.0305.

[166] I.-W. Kim and K. M. Zurek, Flavor and Collider Signatures of Asymmetric Dark Matter, Phys.Rev. D89 (2014), no. 3 035008, [arXiv:1310 .2617].

[167] R. Harnik, J. Kopp, and J. Zupan, Flavor Violating Higgs Decays, JHEP 1303 (2013) 026, [arXiv: 1209.1397].

[168] CMS Collaboration, V. Khachatryan et al., Search for lepton-flavour-violating decays of the Higgs boson, arXiv: 1502.0740.

[169] G. Blankenburg, J. Ellis, and G. Isidori, Flavour-Changing Decays of a $125 \mathrm{GeV}$ Higgs-like Particle, Phys.Lett. B712 (2012) 386-390, [arXiv : 1202 .5704].

[170] A. Goudelis, O. Lebedev, and J.-h. Park, Higgs-induced lepton flavor violation, Phys.Lett. B707 (2012) 369-374, [arXiv:1111.1715].

[171] D. Carmi, A. Falkowski, E. Kuflik, T. Volansky, and J. Zupan, Higgs After the Discovery: A Status Report, JHEP 1210 (2012) 196, [arXiv: 1207.1718].

[172] J. Brod, U. Haisch, and J. Zupan, Constraints on CP-violating Higgs couplings to the third generation, JHEP 1311, 180 (2013) [arXiv: 1310.1385].

[173] F. Goertz, A. Papaefstathiou, L. L. Yang, and J. Zurita, Higgs Boson self-coupling measurements using ratios of cross sections, JHEP 1306 (2013) 016, [arXiv: 1301.3492].

[174] D. E. Ferreira de Lima, A. Papaefstathiou, and M. Spannowsky, Standard model Higgs boson pair production in the $(b \bar{b})(b \bar{b})$ final state, JHEP 1408 (2014) 030, [arXiv: 1404.7139]. 
[175] CMS Collaboration, S. Chatrchyan et al., Search for invisible decays of Higgs bosons in the vector boson fusion and associated ZH production modes, Eur.Phys.J. C74 (2014) 2980, [arXiv:1404.1344].

[176] ATLAS Collaboration, G. Aad et al., Search for Invisible Decays of a Higgs Boson Produced in Association with a Z Boson in ATLAS, Phys.Rev.Lett. 112 (2014) 201802, [arXiv: 1402.3244].

[177] M. Endo and Y. Takaesu, Heavy WIMP through Higgs portal at the LHC, Phys.Lett. B743 (2015) 228-234, [arXiv : 1407 .6882].

[178] Fermi-LAT, DES Collaboration, A. Drlica-Wagner et al., Search for Gamma-Ray Emission from DES Dwarf Spheroidal Galaxy Candidates with Fermi-LAT Data, Astrophys.J. (2015) [arXiv: 1503.0263].

[179] M. Cirelli, G. Corcella, A. Hektor, G. Hutsi, M. Kadastik, et al., PPPC 4 DM ID: A Poor Particle Physicist Cookbook for Dark Matter Indirect Detection, JCAP 1103 (2011) 051, [arXiv: 1012.4515].

[180] M. A. Shifman, A. Vainshtein, and V. I. Zakharov, Remarks on Higgs Boson Interactions with Nucleons, Phys.Lett. B78 (1978) 443.

[181] XENON100 Collaboration, E. Aprile et al., Dark Matter Results from 225 Live Days of XENON100 Data, Phys.Rev.Lett. 109 (2012) 181301, [arXiv:1207 . 5988].

[182] A. L. Fitzpatrick, W. Haxton, E. Katz, N. Lubbers, and Y. Xu, The Effective Field Theory of Dark Matter Direct Detection, JCAP 1302 (2013) 004, [arXiv : 1203 . 3542].

[183] S. L. Glashow and S. Weinberg, Natural Conservation Laws for Neutral Currents, Phys.Rev. D15 (1977) 1958.

[184] E. Paschos, Diagonal Neutral Currents, Phys.Rev. D15 (1977) 1966. 
[185] G. F. Giudice and O. Lebedev, Higgs-dependent Yukawa couplings, Phys.Lett. B665 (2008) 79-85, [arXiv: 0804.1753].

[186] L. Randall and R. Sundrum, A Large mass hierarchy from a small extra dimension, Phys.Rev.Lett. 83 (1999) 3370-3373, [hep-ph/9905221].

[187] M. J. Dugan, H. Georgi, and D. B. Kaplan, Anatomy of a Composite Higgs Model, Nucl.Phys. B254 (1985) 299.

[188] H. Georgi, D. B. Kaplan, and P. Galison, Calculation of the Composite Higgs Mass, Phys.Lett. B143 (1984) 152.

[189] D. B. Kaplan, H. Georgi, and S. Dimopoulos, Composite Higgs Scalars, Phys.Lett. B136 (1984) 187.

[190] D. B. Kaplan and H. Georgi, SU(2) x U(1) Breaking by Vacuum Misalignment, Phys.Lett. B136 (1984) 183.

[191] A. Dery, A. Efrati, Y. Nir, Y. Soreq, and V. Susič, Model building for flavor changing Higgs couplings, Phys.Rev. D90 (2014), no. 11 115022, [arXiv: 1408.1371].

[192] A. Dery, A. Efrati, G. Hiller, Y. Hochberg, and Y. Nir, Higgs couplings to fermions: 2HDM with MFV, JHEP 1308 (2013) 006, [arXiv : 1304.6727].

[193] A. Dery, A. Efrati, Y. Hochberg, and Y. Nir, What if $B R(h \rightarrow \mu \mu) / B R(h \rightarrow \tau \tau)$ does not equal $m_{\mu}^{2} / m_{\tau}^{2}$ ? , JHEP 1305 (2013) 039, [arXiv : 1302 . 3229].

[194] S. El Hedri, P. J. Fox, and J. G. Wacker, Exploring the dark side of the top Yukawa, arXiv:1311.6488.

[195] F. Goertz, Indirect Handle on the Down-Quark Yukawa Coupling, Phys.Rev.Lett. 113 (2014), no. 26 261803, [arXiv:1406.0102]. 
[196] J. Aguilar-Saavedra, A Minimal set of top-Higgs anomalous couplings, Nucl.Phys. B821 (2009) 215-227, [arXiv : 0904 . 2387].

[197] CMS Collaboration, Projected Performance of an Upgraded CMS Detector at the LHC and HL-LHC: Contribution to the Snowmass Process, arXiv:1307.7135.

[198] ATLAS Collaboration, Physics at a High-Luminosity LHC with ATLAS, arXiv:1307.7292.

[199] A. Falkowski, C. Gross, and O. Lebedev, A second Higgs from the Higgs portal, arXiv: 1502.0136.

[200] V. Martin-Lozano, J. M. Moreno, and C. B. Park, Resonant Higgs boson pair production in the $h h \rightarrow b \bar{b} W W \rightarrow b \bar{b} \ell^{+} \nu \ell^{-} \bar{\nu}$ decay channel, arXiv:1501.0379.

[201] H. E. Haber and G. L. Kane, The Search for Supersymmetry: Probing Physics Beyond the Standard Model, Phys.Rept. 117 (1985) 75-263.

[202] A. Falkowski, F. Riva, and A. Urbano, Higgs at last, JHEP 1311 (2013) 111, [arXiv: 1303.1812].

[203] B. Grinstein and P. Uttayarat, Carving Out Parameter Space in Type-II Two Higgs Doublets Model, JHEP 1306 (2013) 094, [arXiv: 1304.0028].

[204] T. Gherghetta and A. Pomarol, Bulk fields and supersymmetry in a slice of AdS, Nucl.Phys. B586 (2000) 141-162, [hep-ph/0003129].

[205] Y. Grossman and M. Neubert, Neutrino masses and mixings in nonfactorizable geometry, Phys.Lett. B474 (2000) 361-371, [hep-ph/9912408].

[206] S. J. Huber and Q. Shafi, Fermion masses, mixings and proton decay in a Randall-Sundrum model, Phys.Lett. B498 (2001) 256-262, [hep-ph/0010195]. 
[207] S. J. Huber, Flavor violation and warped geometry, Nucl.Phys. B666 (2003) 269-288, [hep-ph/0303183].

[208] A. Azatov, M. Toharia, and L. Zhu, Higgs Mediated FCNC's in Warped Extra Dimensions, Phys.Rev. D80 (2009) 035016, [arXiv: 0906.1990].

[209] S. Casagrande, F. Goertz, U. Haisch, M. Neubert, and T. Pfoh, Flavor Physics in the Randall-Sundrum Model: I. Theoretical Setup and Electroweak Precision Tests, JHEP 0810 (2008) 094, [arXiv: 0807.4937$].$

[210] M. Bauer, S. Casagrande, U. Haisch, and M. Neubert, Flavor Physics in the Randall-Sundrum Model: II. Tree-Level Weak-Interaction Processes, JHEP 1009 (2010) 017, [arXiv: 0912.1625].

[211] R. Malm, M. Neubert, K. Novotny, and C. Schmell, 5D perspective on Higgs production at the boundary of a warped extra dimension, JHEP 1401 (2014) 173, [arXiv: 1303.5702].

[212] P. R. Archer, M. Carena, A. Carmona, and M. Neubert, Higgs Production and Decay in Models of a Warped Extra Dimension with a Bulk Higgs, JHEP 1501 (2015) 060, [arXiv: 1408.5406].

[213] M. Blanke, A. J. Buras, B. Duling, S. Gori, and A. Weiler, $\Delta F=2$ Observables and Fine-Tuning in a Warped Extra Dimension with Custodial Protection, JHEP 0903 (2009) 001, [arXiv: 0809.1073].

[214] M. Blanke, A. J. Buras, B. Duling, K. Gemmler, and S. Gori, Rare K and B Decays in a Warped Extra Dimension with Custodial Protection, JHEP 0903 (2009) 108, [arXiv: 0812.3803]. 
[215] M. E. Albrecht, M. Blanke, A. J. Buras, B. Duling, and K. Gemmler, Electroweak and Flavour Structure of a Warped Extra Dimension with Custodial Protection, JHEP 0909 (2009) 064, [arXiv : 0903 . 2415].

[216] K. Agashe, G. Perez, and A. Soni, Collider Signals of Top Quark Flavor Violation from a Warped Extra Dimension, Phys.Rev. D75 (2007) 015002, [hep-ph/0606293].

[217] K. Agashe, A. Azatov, Y. Cui, L. Randall, and M. Son, Warped Dipole Completed, with a Tower of Higgs Bosons, arXiv: 1412.6468.

[218] B. M. Dillon and S. J. Huber, Non-Custodial Warped Extra Dimensions at the LHC?, JHEP 1506 (2015) 066, [arXiv: 1410 .7345].

[219] G. Cacciapaglia, C. Csaki, J. Galloway, G. Marandella, J. Terning, et al., A GIM Mechanism from Extra Dimensions, JHEP 0804 (2008) 006, [arXiv : 0709.1714].

[220] K. Agashe, G. Perez, and A. Soni, Flavor structure of warped extra dimension models, Phys.Rev. D71 (2005) 016002, [hep-ph/0408134].

[221] K. Agashe, G. Perez, and A. Soni, B-factory signals for a warped extra dimension, Phys.Rev.Lett. 93 (2004) 201804, [hep-ph/0406101].

[222] A search for $\mathbf{t} \overline{\mathbf{t}}$ resonances using lepton plus jets events in proton-proton collisions at $\sqrt{s}=8 \mathrm{TeV}$ with the ATLAS detector, Tech. Rep. ATLAS-CONF-2015-009, CERN, Geneva, Mar, 2015.

[223] D. B. Kaplan, Flavor at SSC energies: A New mechanism for dynamically generated fermion masses, Nucl.Phys. B365 (1991) 259-278.

[224] K. Agashe and R. Contino, Composite Higgs-Mediated FCNC, Phys.Rev. D80 (2009) 075016, [arXiv : 0906.1542]. 
[225] M. Gillioz, R. Grober, C. Grojean, M. Muhlleitner, and E. Salvioni, Higgs Low-Energy Theorem (and its corrections) in Composite Models, JHEP 1210 (2012) 004, [arXiv: 1206.7120].

[226] C. Delaunay, C. Grojean, and G. Perez, Modified Higgs Physics from Composite Light Flavors, JHEP 1309 (2013) 090, [arXiv : 1303. 5701].

[227] A. Pomarol and F. Riva, The Composite Higgs and Light Resonance Connection, JHEP 1208 (2012) 135, [arXiv: 1205.6434].

[228] A. Azatov, G. Panico, G. Perez, and Y. Soreq, On the Flavor Structure of Natural Composite Higgs Models Eamp; Top Flavor Violation, JHEP 1412 (2014) 082, [arXiv: 1408.4525].

[229] M. Redi and A. Weiler, Flavor and CP Invariant Composite Higgs Models, JHEP 1111 (2011) 108, [arXiv: 1106 . 6357].

[230] M. Redi, Composite MFV and Beyond, Eur.Phys.J. C72 (2012) 2030, [arXiv: 1203.4220].

[231] A. Geringer-Sameth, M. G. Walker, S. M. Koushiappas, S. E. Koposov, V. Belokurov, et al., Evidence for Gamma-ray Emission from the Newly Discovered Dwarf Galaxy Reticulum 2, arXiv: 1503.0232.

[232] J. Alwall, R. Frederix, S. Frixione, V. Hirschi, F. Maltoni, et al., The automated computation of tree-level and next-to-leading order differential cross sections, and their matching to parton shower simulations, JHEP 1407 (2014) 079, [arXiv: 1405. 0301].

[233] CMS Collaboration, S. Chatrchyan et al., Observation of a new boson at a mass of $125 \mathrm{GeV}$ with the CMS experiment at the LHC, Phys.Lett. B716 (2012) 30-61, [arXiv:1207.7235]. 
[234] ATLAS Collaboration, G. Aad et al., Observation of a new particle in the search for the Standard Model Higgs boson with the ATLAS detector at the LHC, Phys.Lett. B716 (2012) 1-29, [arXiv: 1207.7214].

[235] M. Voloshin, CP Violation in Higgs Diphoton Decay in Models with Vectorlike Heavy Fermions, Phys. Rev. D 86 (2012) 093016, [arXiv: 1208 .4303].

[236] Y. Chen and R. Vega-Morales, Extracting Effective Higgs Couplings in the Golden Channel, arXiv:1310.2893.

[237] I. Anderson, S. Bolognesi, F. Caola, Y. Gao, A. V. Gritsan, et al., Constraining anomalous HVV interactions at proton and lepton colliders, arXiv:1309.4819.

[238] Y. Sun, X.-F. Wang, and D.-N. Gao, CP mixed property of the Higgs-like particle in the decay channel $h \rightarrow Z Z^{*} \rightarrow 4 l$, arXiv:1309.4171.

[239] R. Boughezal, T. J. LeCompte, and F. Petriello, Single-variable asymmetries for measuring the 'Higgs' boson spin and CP properties, arXiv: 1208.4311.

[240] S. Bolognesi, Y. Gao, A. V. Gritsan, K. Melnikov, M. Schulze, et al., On the spin and parity of a single-produced resonance at the LHC, Phys.Rev. D86 (2012) 095031, [arXiv: 1208.4018].

[241] R. Harnik, A. Martin, T. Okui, R. Primulando, and F. Yu, Measuring CP Violation in $h \rightarrow \tau^{+} \tau^{-}$at Colliders, Phys.Rev. D88 (2013) 076009, [arXiv: 1308.1094].

[242] S. Berge, W. Bernreuther, and H. Spiesberger, Higgs CP properties using the tau decay modes at the ILC, arXiv:1308.2674.

[243] J. R. Dell'Aquila and C. A. Nelson, Usage of the $\bar{\tau} \tau$ or T Anti-t Decay Mode to Distinguish an Intermediate Mass Higgs Boson From a Technipion, Nucl.Phys. B320 (1989) 86-102. 
[244] J. R. Dell'Aquila and C. A. Nelson, CP Determination for New Spin Zero Mesons by the $\bar{\tau} \tau$ Decay Mode, Nucl.Phys. B320 (1989) 61.

[245] B. Grzadkowski and J. Gunion, Using decay angle correlations to detect CP violation in the neutral Higgs sector, Phys.Lett. B350 (1995) 218-224, [hep-ph/9501339].

[246] G. Bower, T. Pierzchala, Z. Was, and M. Worek, Measuring the Higgs boson's parity using tau —i rho nu, Phys.Lett. B543 (2002) 227-234, [hep-ph/0204292].

[247] M. Worek, Higgs CP from H / A0 — i tau tau decay, Acta Phys.Polon. B34 (2003) 4549-4560, [hep-ph/0305082].

[248] K. Desch, Z. Was, and M. Worek, Measuring the Higgs boson parity at a linear collider using the tau impact parameter and tau - i rho nu decay, Eur.Phys.J. C29 (2003) 491-496, [hep-ph/0302046].

[249] S. Berge and W. Bernreuther, Determining the CP parity of Higgs bosons at the LHC in the tau to 1-prong decay channels, Phys.Lett. B671 (2009) 470-476, [arXiv: 0812.1910].

[250] S. Berge, W. Bernreuther, and J. Ziethe, Determining the CP parity of Higgs bosons at the LHC in their tau decay channels, Phys.Rev.Lett. 100 (2008) 171605, [arXiv: 0801.2297].

[251] S. Berge, W. Bernreuther, B. Niepelt, and H. Spiesberger, How to pin down the $C P$ quantum numbers of a Higgs boson in its tau decays at the LHC, Phys.Rev. D84 (2011) 116003, [arXiv:1108.0670].

[252] C. N. Yang, Selection rules for the dematerialization of a particle into two photons, Phys. Rev. 77 (Jan, 1950) 242-245.

[253] C. N. Yang, Possible experimental determination of whether the neutral meson is scalar or pseudoscalar, Phys. Rev. 77 (Mar, 1950) 722-723. 
[254] N. M. Kroll and W. Wada, Internal pair production associated with the emission of high-energy gamma rays, Phys. Rev. 98 (Jun, 1955) 1355-1359.

[255] Y. Grossman and D. Pirjol, Extracting and using photon polarization information in radiative B decays, JHEP 0006 (2000) 029, [hep-ph/0005069].

[256] ATLAS Collaboration, G. Aad et al., Search for the Standard Model Higgs boson in the diphoton decay channel with $4.9 \mathrm{fb}^{-1}$ of pp collisions at $\sqrt{s}=7 \mathrm{TeV}$ with ATLAS, Phys.Rev.Lett. 108 (2012) 111803, [arXiv:1202 .1414].

[257] CMS Collaboration, S. Chatrchyan et al., Search for the standard model Higgs boson decaying into two photons in pp collisions at $\sqrt{s}=7 \mathrm{TeV}$, Phys.Lett. B710 (2012) 403-425, [arXiv: 1202.1487].

[258] W. N. Cottingham and I. B. Whittingham, Signals of higgs sector CP violation in neutral higgs boson decays, Phys. Rev. D 52 (Jul, 1995) 539-542.

[259] ATLAS Collaboration, Measurements of the properties of the higgs-like boson in the four lepton decay channel with the atlas detector, Tech. Rep.

ATLAS-CONF-2013-013, CERN, 2013.

[260] CMS Collaboration, S. Chatrchyan et al., Study of the mass and spin-parity of the higgs boson candidate via its decays to z boson pairs, Phys.Rev.Lett. 110 (2013) 081803, [arXiv:1212.6639].

[261] G. Belanger, B. Dumont, U. Ellwanger, J. Gunion, and S. Kraml, Global fit to Higgs signal strengths and couplings and implications for extended Higgs sectors, arXiv:1306.2941.

[262] A. Czarnecki and B. Krause, On the dipole moments of fermions at two loops, Acta Phys.Polon. B28 (1997) 829-834, [hep-ph/9611299]. 
[263] D. McKeen, M. Pospelov, and A. Ritz, Modified Higgs branching ratios versus CP and lepton flavor violation, Phys.Rev. D86 (2012) 113004, [arXiv: 1208.4597].

[264] ACME Collaboration, Order of Magnitude Smaller Limit on the Electric Dipole Moment of the Electron, arXiv: 1310.7534.

[265] J. Fan and M. Reece, Probing Charged Matter Through Higgs Diphoton Decay, Gamma Ray Lines, and EDMs, JHEP 1306 (2013) 004, [arXiv: 1301 .2597].

[266] J. Shu and Y. Zhang, Impact of a CP Violating Higgs: from LHC to Baryogenesis, Phys.Rev.Lett. 111 (2013) 091801, [arXiv: 1304.0773].

[267] A. Y. Korchin and V. A. Kovalchuk, Polarization effects in the Higgs boson decay to gamma Z and test of CP and CPT symmetries, Phys.Rev. D88 (2013) 036009, [arXiv: 1303.0365].

[268] A. Y. Korchin and V. Kovalchuk, Higgs Boson Decay to $\gamma Z$ and Test of CP and CPT Symmetries, Acta Phys.Polon. B44 (2013), no. 11 2121-2128.

[269] W.-Y. Keung, I. Low, and J. Shu, Landau-Yang Theorem and Decays of a Z' Boson into Two Z Bosons, Phys.Rev.Lett. 101 (2008) 091802, [arXiv: 0806 .2864].

[270] Q.-H. Cao, C. Jackson, W.-Y. Keung, I. Low, and J. Shu, The Higgs Mechanism and Loop-induced Decays of a Scalar into Two Z Bosons, Phys.Rev. D81 (2010) 015010, [arXiv: 0911.3398].

[271] M. Berger, J. Hubbell, S. Seltzer, J. Chang, J. Coursey, R. Sukumar, D. Zucker, and K. Olsen, "XCOM: Photon cross section database version 1.5." National Institute of Standards and Technology, 2010.

[272] Y.-S. Tsai, Pair production and bremsstrahlung of charged leptons, Rev. Mod. Phys. 46 (Oct, 1974) 815-851. 
[273] Y.-S. Tsai, Erratum: Pair production and bremsstrahlung of charged leptons, Rev. Mod. Phys. 49 (Apr, 1977) 421-423.

[274] CMS Collaboration, Updated measurements of the Higgs boson at $125 \mathrm{GeV}$ in the two photon decay channel, Tech. Rep. CMS-PAS-HIG-13-001, CERN, Geneva, 2013.

[275] ATLAS Collaboration, Measurements of the properties of the Higgs-like boson in the two photon decay channel with the ATLAS detector using $25 \mathrm{fb}^{-1}$ of proton-proton collision data, Tech. Rep. ATLAS-CONF-2013-012, CERN, Geneva, Mar, 2013.

[276] S. Drell and J. Walecka, Electrodynamic processes with nuclear targets, Annals of Physics 28 (1964), no. $118-33$.

[277] Particle Data Group Collaboration, J. Beringer et al., The review of particle physics, Phys. Rev. D 86 (2012) 010001.

[278] ATLAS Collaboration, G. Aad et al., The atlas collaboration, JINST 3 (2008), no. 08 S08003.

[279] J. C. Slater, Atomic radii in crystals, The Journal of Chemical Physics 41 (1964), no. 10 3199-3204.

[280] L. Schiff, Energy-angle distribution of thin target bremsstrahlung, Physical Review 83 (1951), no. 2252.

[281] D0 Collaboration, V. Abazov et al., A precision measurement of the mass of the top quark, Nature 429 (2004) 638-642, [hep-ex/0406031].

[282] D0 Collaboration, V. Abazov et al., Helicity of the w boson in lepton + jets events, Physics Letters B 617 (2005), no. 1-2 1-10.

[283] P. Artoisenet, V. Lemaitre, F. Maltoni, and O. Mattelaer, Automation of the matrix element reweighting method, JHEP 1012 (2010) 068, [arXiv: 1007.3300]. 
[284] R. H. Dalitz and G. R. Goldstein, Decay and polarization properties of the top quark, Phys. Rev. D 45 (Mar, 1992) 1531-1543.

[285] F. Fiedler, A. Grohsjean, P. Haefner, and P. Schieferdecker, The Matrix Element Method and its Application in Measurements of the Top Quark Mass, Nucl.Instrum.Meth. A624 (2010) 203-218, [arXiv: 1003.1316].

[286] K. Kondo, Dynamical likelihood method for reconstruction of events with missing momentum. ii. mass spectra for $2 \rightarrow 2$ processes, Journal of the Physical Society of Japan 60 (1991), no. 3 836-844.

[287] R. Tanaka, C. Mariotti, S. Heinemeyer, and G. Passarino, "Lhc higgs cross section working group: Recommended values on sm higgs xs at 7/8/14/33/...100 tev for hl-lhc and he-lhc."

[288] S. Dawson, A. Gritsan, H. Logan, J. Qian, C. Tully, et al., Higgs Working Group Report of the Snowmass 2013 Community Planning Study, arXiv:1310.8361.

[289] M. Gronau, Y. Grossman, D. Pirjol, and A. Ryd, Measuring the photon polarization in B -Eggt; K pi pi gamma, Phys.Rev.Lett. 88 (2002) 051802, [hep-ph/0107254].

[290] M. Gronau and D. Pirjol, Photon polarization in radiative B decays, Phys.Rev. D66 (2002) 054008, [hep-ph/0205065].

[291] LHCb Collaboration, R. Aaij et al., Observation of Photon Polarization in the $b \rightarrow s \gamma$ Transition, Phys.Rev.Lett. 112 (2014), no. 16 161801, [arXiv: 1402.6852].

[292] T. Mannel and S. Recksiegel, Probing the helicity structure of $b-i$ s gamma in Lambda(b) - i Lambda gamma, Acta Phys.Polon. B28 (1997) 2489-2493, [hep-ph/9710287].

[293] G. Hiller and A. Kagan, Probing for new physics in polarized Lambda(b) decays at the Z, Phys.Rev. D65 (2002) 074038, [hep-ph/0108074]. 
[294] D. Atwood, M. Gronau, and A. Soni, Mixing induced CP asymmetries in radiative B decays in and beyond the standard model, Phys.Rev.Lett. 79 (1997) 185-188, [hep-ph/9704272].

[295] F. Kruger, L. M. Sehgal, N. Sinha, and R. Sinha, Angular distribution and CP asymmetries in the decays $\bar{B} \rightarrow K^{-} \pi^{+} e^{-} e^{+}$and $\bar{B} \rightarrow \pi^{-} \pi^{+} e^{-} e^{+}$, Phys.Rev. D61 (2000) 114028, [hep-ph/9907386].

[296] C. Kim, Y. G. Kim, C.-D. Lu, and T. Morozumi, Azimuthal angle distribution in $B \rightarrow K^{*}(\rightarrow K \pi) \ell^{+} \ell^{-}$at low invariant $m\left(\ell^{+} \ell^{-}\right)$region, Phys.Rev. D62 (2000) 034013, [hep-ph/0001151].

[297] D. Melikhov, N. Nikitin, and S. Simula, Probing right-handed currents in B - Egt; K* lepton+ lepton- transitions, Phys.Lett. B442 (1998) 381-389, [hep-ph/9807464].

[298] B. Grinstein, Y. Grossman, Z. Ligeti, and D. Pirjol, The Photon polarization in B —Egt; X gamma in the standard model, Phys.Rev. D71 (2005) 011504, [hep-ph/0412019].

[299] F. Bishara, Y. Grossman, R. Harnik, D. J. Robinson, J. Shu, et al., Probing CP Violation in $h \rightarrow \gamma \gamma$ with Converted Photons, JHEP 1404 (2014) 084, [arXiv: 1312.2955].

[300] BaBar Collaboration, D. Boutigny et al., BaBar technical design report, .

[301] LHCb Collaboration, R. Aaij et al., LHCb Detector Performance, Int.J.Mod.Phys. A30 (2015) 1530022, [arXiv: 1412.6352].

[302] Belle II Collaboration, T. Abe et al., Belle II Technical Design Report, arXiv: 1011.0352. 
[303] D. Bernard, HARPO-A gaseous TPC for high angular resolution $\gamma$-ray astronomy and polarimetry from the MeV to the TeV, Nuclear Instruments and Methods in Physics Research A 718 (Aug., 2013) 395-399, [arXiv: 1210 .4399].

[304] J. W. MOTZ, H. A. OLSEN, and H. W. KOCH, Pair production by photons, Rev. Mod. Phys. 41 (Oct, 1969) 581-639.

[305] H. K. Dreiner, H. E. Haber, and S. P. Martin, Two-component spinor techniques and Feynman rules for quantum field theory and supersymmetry, Phys.Rept. 494 (2010) 1-196, [arXiv: 0812.1594].

[306] M. D'Onofrio, K. Rummukainen, and A. Tranberg, The sphaleron rate at the electroweak crossover with $125 \mathrm{GeV}$ Higgs mass, PoS LATTICE2012 (2012) 055, [arXiv:1212.3206].

[307] J. A. Harvey and M. S. Turner, Cosmological baryon and lepton number in the presence of electroweak fermion number violation, Phys.Rev. D42 (1990) 3344-3349.

[308] B. Feldstein and A. L. Fitzpatrick, Discovering Asymmetric Dark Matter with Anti-Neutrinos, JCAP 1009 (2010) 005, [arXiv: 1003 . 5662].

[309] Y. Burnier, M. Laine, and M. Shaposhnikov, Baryon and lepton number violation rates across the electroweak crossover, JCAP 0602 (2006) 007, [hep-ph/0511246].

[310] C. Degrande, C. Duhr, B. Fuks, D. Grellscheid, O. Mattelaer, et al., UFO - The Universal FeynRules Output, Comput.Phys.Commun. 183 (2012) 1201-1214, [arXiv: 1108.2040].

[311] L. J. Dixon, Calculating scattering amplitudes efficiently, hep-ph/9601359.

[312] L. J. Dixon, E. N. Glover, and V. V. Khoze, MHV rules for Higgs plus multi-gluon amplitudes, JHEP 0412 (2004) 015, [hep-th/0411092]. 
[313] T. Hahn, CUBA: A Library for multidimensional numerical integration, Comput.Phys.Commun. 168 (2005) 78-95, [hep-ph/0404043].

[314] N. L. Johnson, S. Kotz, and N. Balakrishnan, Continuous Univariate Distributions, Vol. 1. Wiley-Interscience, second ed., 1994. 